\title{
MASTER
}

LA--10802-MS

$\mathrm{DE} 87 \quad 003802$

\section{Chemistry of Diagenetically Altered Tuffs at a Potential Nuclear Waste Repository, Yucca Mountain, Nye County, Nevada}

\author{
David E. Broxton \\ Richard G. Warren \\ Roland C. Hagan \\ Gary Luedemann
}

\begin{abstract}
DISCLAIMER
This report was prepared as an account of work sponsored by ari agency of the United States Government. Neither the United States Government nor any agency thereof, nor any of their employees, makes any warrarty, express or implied, or assumes any legal liability or responsibility for the accuracy, completeness, or usefulness of any information, apparatus, product, or process disclosed, or represents that its use would not infringe privately owned rights. Refer. ence herein to any specific commercial product, process, or service by trade name, trademark, manufacturer, or otherwise does nol necessarily constitute or imply its endorsement, zecommendation, or favoring by the United States Government or any agency thereof. The views and opinions of authors expressed herein do not necessarily state or reflect those of the United States Government or any agency thereof.
\end{abstract}


ABSTRACT . . . . . . . . . . . . . . . . . . . . 1

I. INTRODUCTION . . . . . . . . . . . . . . . . . . . 2

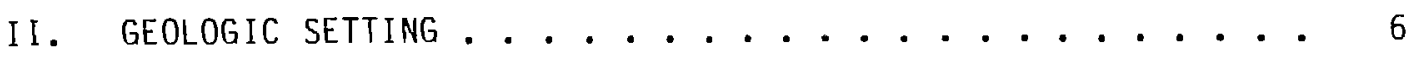

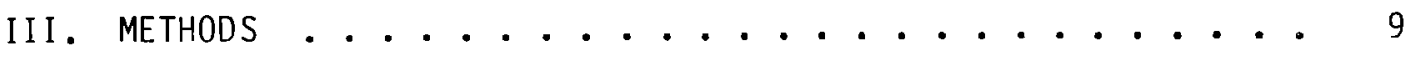

A. Whole-Rock Compositions by X-Ray Fluorescence, Atomic Abserption, and Emission Spectrophotometry . . . . . 9

B. Whole-Rock Compositions by Automated Neutron Activation Analysis ................ 12

C. Mineral and Glass Compositions Determined by Electron Microprobe . . . . . . . . . . . 13

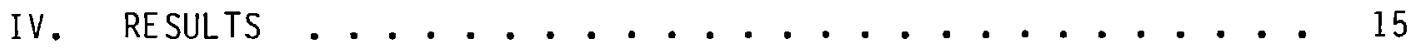

V. DISCUSSION . . . . . . . . . . . . . . . . . . 31

VI. SUMMARY AND CONCLUSIONS . . . . . . . . . . . . . . . . . . . 34

ACKNOWLEOGMENTS . . . . . . . . . . . . . . . . 35

REFERENCES . . . . . . . . . . . . . . . . . 35

APPENDIX A: MAJOR-ELEMENT ANALYSES OF TUFFS BY $X$-RAY

FLUORESCENCE, YUCCA MOUNTAIN, NEVADA . . . . . . . . . . 39

APPENDIX $B$ : COMPARISON OF ANALYSES DETERMINED BY X-RAY

FLUORESCENCE (XRF), ATOMIC ABSORPTION (AA), AND EMISSION

SPECTROPHOTOMETRY (ES) FOR DRILL CORE SAMPLES FROM

YUCCA MOUNTAIN, NEVADA . . . . . . . . . . . . . . . . 49

APPENDIX C: MAJOR-AND TRACE-ELEMENT CONCENTRATIONS DETERMINED BY

NEUTRON ACTIVATION ANALYSIS IN WHOLE-ROCK SAMPLES FROM DRILL

CORE IN DRILL HOLES USW G-1, USW G-2, USW GU-3, AND FROM

TUFF OF CALICO HILLS SAMPLES COLIECTED IN OUTCROP AT PROW

PASS, NORTHERN YUCCA MOUNTAIN . . . . . . . . . . . . . 52

APPENDIX D: ANALYTICAL VALUES FOR NBS STANDARDS BY AUTOMATED

NEUTRON ACTIVATION ANALYSIS . . . . . . . . . . . . . . 60

APPENDIX E: GLASS COMPOSITIONS DETERMINED BY ELECTRON MICROPROBE

FOR TUFFS OF YUCCA MOUNTAIN, NEVADA . . . . . . . . . . . 61

APPENDIX F: CLINOPTILOLITE COMPOSITIONS DETERMINED BY ELECTRON

MICROPROBE, YUCCA MOUNTAIN, NEVADA . . . . . . . . . . . 79

APPENDIX G: ANALCIME COMPOSITIONS DETERMINED BY ELECTRON

MICROPROBE, YUCCA MOUNTAIN, NEVADA . . . . . . . . . . . . 137 
APPENDIX H: AUTHIGENIC FELDSPAR COMPOSITIONS DETERMINED BY ELECTRON MICROPROBE, YUCCA MOUNTAIN, NEVADA . . . . . .

\section{FIGURES}

1. Location and generalized geologic map of Yucca Mountain, Nevada, showing location of drill holes (adapted from Scott and Castellanos 1984). ............ 3

2. Fence diagram showing distribution of principal zeolitic tuffs at Yucca Mountain, Nevada.............44

3. Schematic cross section of Yucca Mountain, Nevada, showing position of potentiai repository and principal zeolitic tuffs. 5

4. Comparison of whole-rock analyses determined by $x$-ray fluorescence vs. analyses by atomic absorption and emission spectrophotometry. Solid diagonal lines have a slope of one, indicating perfect correlations between analytical methods. Dashed diagonal lines represent percent deviation of $x$-ray fluorescence analysis from atomic absorption and emission spectrophotometry analyses. .............

5. Semilogarithmic plot showing sodium count rates as a function of time for a variety of zeolites. Analyses were performed using a stationary electron microprobe beam with an accelerating potential of $15 \mathrm{keV}$. The beam was rastered with a $20-\mu$ m edge. . . . . . . . . . . .

6. Logarithmic plot showing eiemental ratios, normalized to equal aluminum contents, for zeolitic and unaltered tuffs, Yucca Mountain, Nevada. ..............

7. Triangular diagrams showing relative alkali and alkaline-earth contents for whole-rock samples from diagenetic Zones I and II. . . . . . . . . . . .

8. Triangular diagram showing relative alkali and alkaline-earth contents for qlasses from diagenetic Zone I, Yucca Mountain, Nevada.............

9. Triangular diagram showing relative alkali and aikaline-earth contents for clinoptilolites of diagenetic Zone I, Yucca Mountain, Nevada. . . . . . 25

10. Histogram comparing Si/Al ratios for clinoptilolites of Yucca Mountain, Nevada. ............

11. Trianqular diagrams showing relative alkali and alkaline-earth contents for clinoptilolites of diagenetic Zones II and III, Yucca Mountain, Nevada. . . . 
12. Triangular diagram showing re ative alkali and

alkaline-earth contents for cinoptiloiltes in the

tuff of Calico Hills at Prow lass, northern

Yucca Mountain. ...............

13. Histogram showing distribution of si/Al ratios for

analcimes, Yucca Mountain, Nelada. . . . . . . 30

14. Histoqram showing distributior for sum of cations in nontetrahedral sites within al thigenic potassium

feldspar, Yucca Mountain, Nevada.......... 30

15. Triangular diagram showing alkali and alkaline-earth contents for clinoptilolites in the unsaturated zone below the exploration block, Yucca Mountain, Nevada.

TABLES

I. STRATIGRAPHY OF VOLCANIC UNITS AT YUCCA MOUNTAIN, NEVADA .....................

II. REPRESENTATIVE WHOLE-ROCK COMPOISITIONS FOR TUFFS AT YUCCA MOUNTAIN, NEVADA ............

III. REPRESENTATIVE ANALYSES OF CLINOPTILOLITES DETERMINED BY ELECTRON MICROPROBE, YUCCA MOUNTAIN, NEVADA . . .

IV. COMPARISON OF SELECTED TRACE ELLMMENTS BETWEEN UNAL TERED AND ALTERED TUFFS IN DIAGENE IIIC ZONE 1 , 
CHEMISTRY OF DIAGENETICALLY AL TERED TUFFS AT A POTENTIAL NUCLEAR WASTE REPOSITORY, YUCCA MOUNTAIN, NYE COUNTY, NEVADA

by

David E. Broxton, Richard G. Warren, Roland C. Hagan, and Gary Luedemann

\section{ABSTRACT}

This report describes the chemistry of diagenetically altered tuffs at a Dotential nuclear waste repository, Yucca Mountain, Nevada. These tuffs contain substantial amounts of zeolites that are highly sorptive of certain radionuclides. Because of their widespread distribution, the zeolitic tuffs could provide important barriers to radionuclide migration. Physical properties of these tuffs and of their constituent zeolites are influenced by their chemical compositions. This study defines the amount of chemical variability within diagenetically altered tuffs and within diagenetic minerals at Yucca Mountain.

Major- and trace-element compositions in whole-rock samples were determined by a combination of $x$-ray fluorescence, neutron activation, atomic absorption, and plasma-source emission spectrophotometry methods. Glass, clinoptilolite, analcime, and authigenic feldspar compositions were determined by electron microprobe. Mordenite, an important zeolite at Yucca Mountain, is too fine grained and intergrown with other diagenetic minerals for accurate determinations of compositions. New whole-rock and mineral compositions as well as previously published analyses for mineral chemistry are summarized in the appendices of this redort.

Zeolitic tuffs at Yucca Mountain formed by diagenetic al teration of rhyolitic vitric tuffs. Despite their similar starting compositions, these tuffs developed compositions that vary both vertically and laterally. Widespread chemical variations were the result of open-system chemical diagenesis in which chemical components of the tuffs were mobilized and redistributed by groundwaters. Alkalies, alkaline earths, and silica were the most mobile elements during diagenesis.

The zeolitic tuffs can be divided into three compositional groups: (1) calcium- and magnesium-rich tuffs associated with relatively thin zones of alteration in the unsaturated zone, (2) tuffs in thick zones of alteration at and below the water table that grade laterally from sodic compositions on the western side of Yucca Mountain to calcic compositions on the eastern side, and (3) potassic tuffs at the north end of Yucca Mountain. Similar chemical variations 
are found in the clinoptilolites that make up these tuffs. Other diagenetic minerals such as analcime, potassium feldspar, and albite have uniform compositions.

Physical properties of tuffs and their constituent zeolites at Yucca Mountain may be affected by variations in compositions. Properties important for assessment of repository performance include behavior and ion exchange. The data presented in this report can be used in conjunction with experimental data to model the behavior of Yucca Mountain tuffs surrounding a high-level nuclear waste repository.

\section{INTRODUCTION}

Yucca Mountain is located along the southwest border of the Nevada Test Site in south-central Nevada (Fig. 1). The U.S. Debartment of Energy (DOE) and its subcontractors are conducting detailed studies of the ash-flow and bedded tuffs at Yucca Mountain to determine the suitability of the tuffs as a high-level nuclear waste repository. These studies are supported by the Nevada Nuclear Waste Storage Investigations (NNWSI) Project as part of the DOE Civilian Radioactive Waste Management Program. The NNWSI Project is managed by the Waste Management Project Office of the DDE Nevada Operations Office.

The presence of thick zeolitic tuffs in continuous zones throughout the region (Fig. 2) was a major consideration for choosing Yucca Mountain as a potential repository site (Johnstone and Wolfsberg 1980). The zeolites could provide important geologic barriers to nuclear waste migration because they can sorb some important radionuclides from groundwaters and thus retard movement of radioactivity away from the repository site. The potential repository horizon is sited within the unsaturated zone in the lower half of the Topopah Spring Member of the Paintbrush Tuff (Fig. 3). The repository host rock is a densely welded and devitrified tuff consisting predominantly of alkali feldspars and silica minerals. Below, zeolitic tuffs occur within several stratigraphic intervals between the redository and the water table, providing probabie barriers to downward radionuclide migration in the unsaturated zone. Additional zeolitic tuffs below the water table provide potential barriers to lateral radionuclide migration through the saturated zone. 


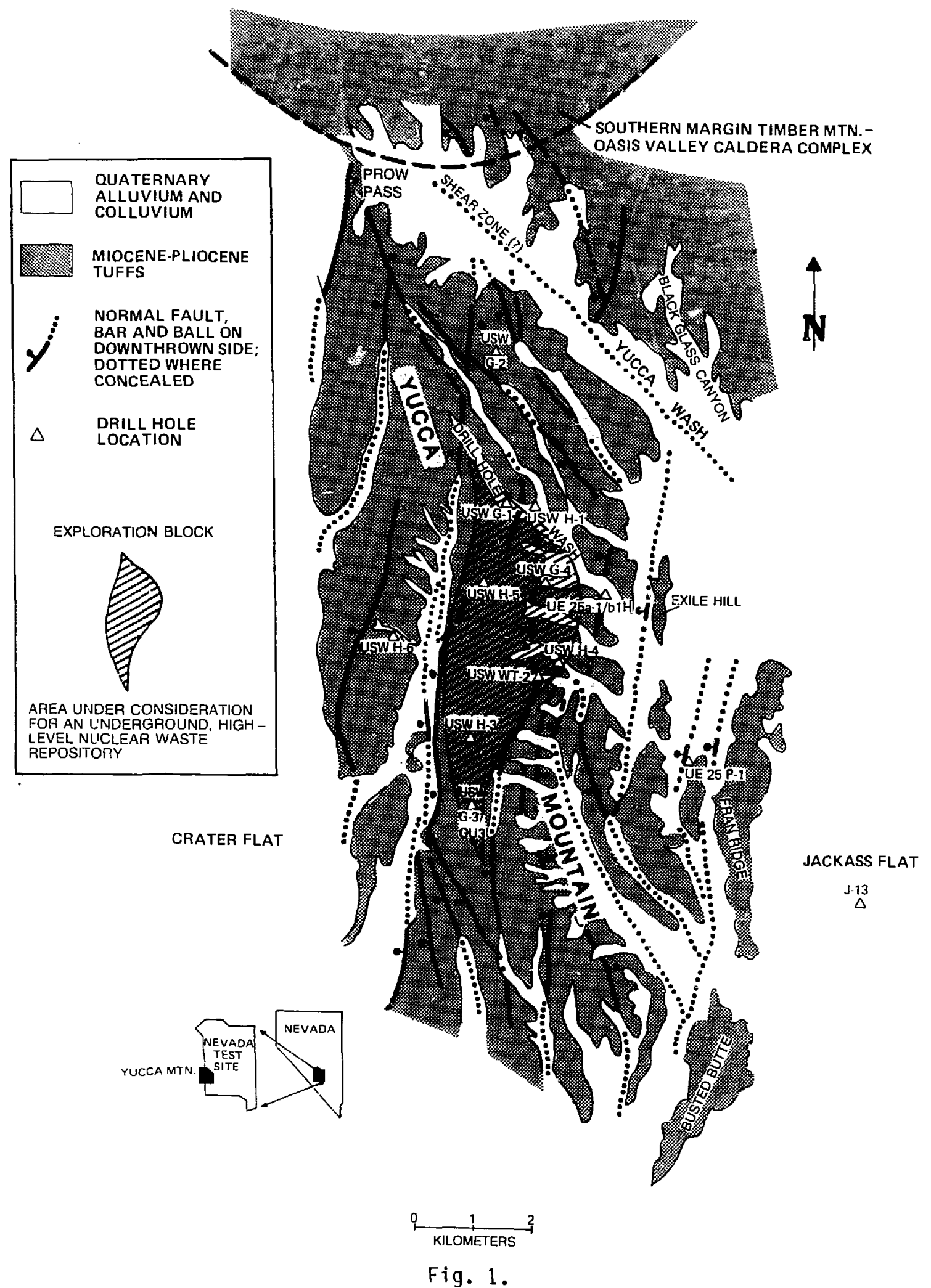

Location and generalized geologic map of Yucca Mountain, Nevacia, showing location of drill holes (adapted from Scott and Castellanos 1984). 


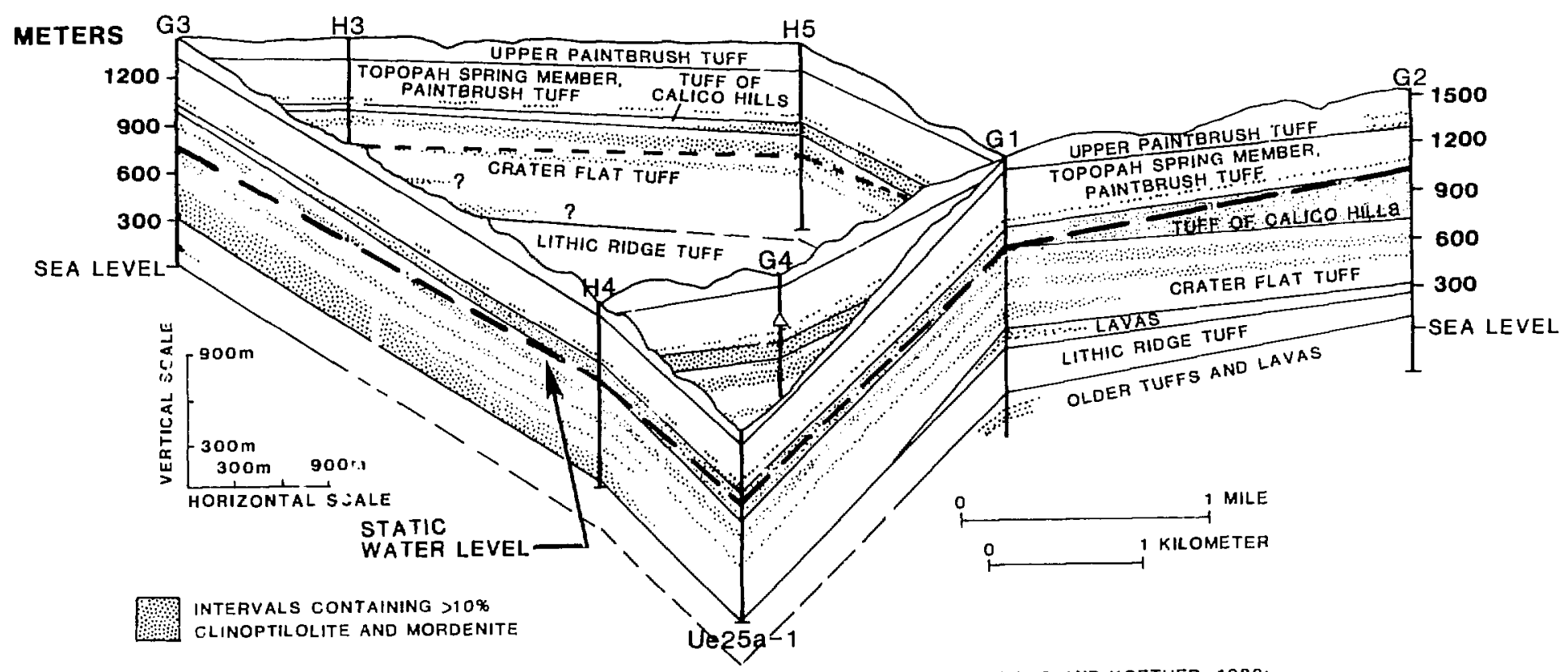

STRATIGRAPHIC CONTACTS FROM SPENGLER ol al. 1879: SPENGLER el al, 1881: MALDONADO AND KOETHER. 1983:

SCOTT AND CASTELLANOS, 1983. PRELIMINARY STRATIGRAPHIC CONTACTS FOR DRILL HOLES H3, H4, H5, AND G4 PROVIDED BY SPENGLER (PERSONAL COMMUNICATION, 1983). STATIC WATER LEVEL DATA FROM NUCLEAR HYDROLOGY GROUP, USGS.

Fig. 2 .

Fence diagram showing distribution of principal zeolitic tuffs at Yucca Mountain, Nevada. 


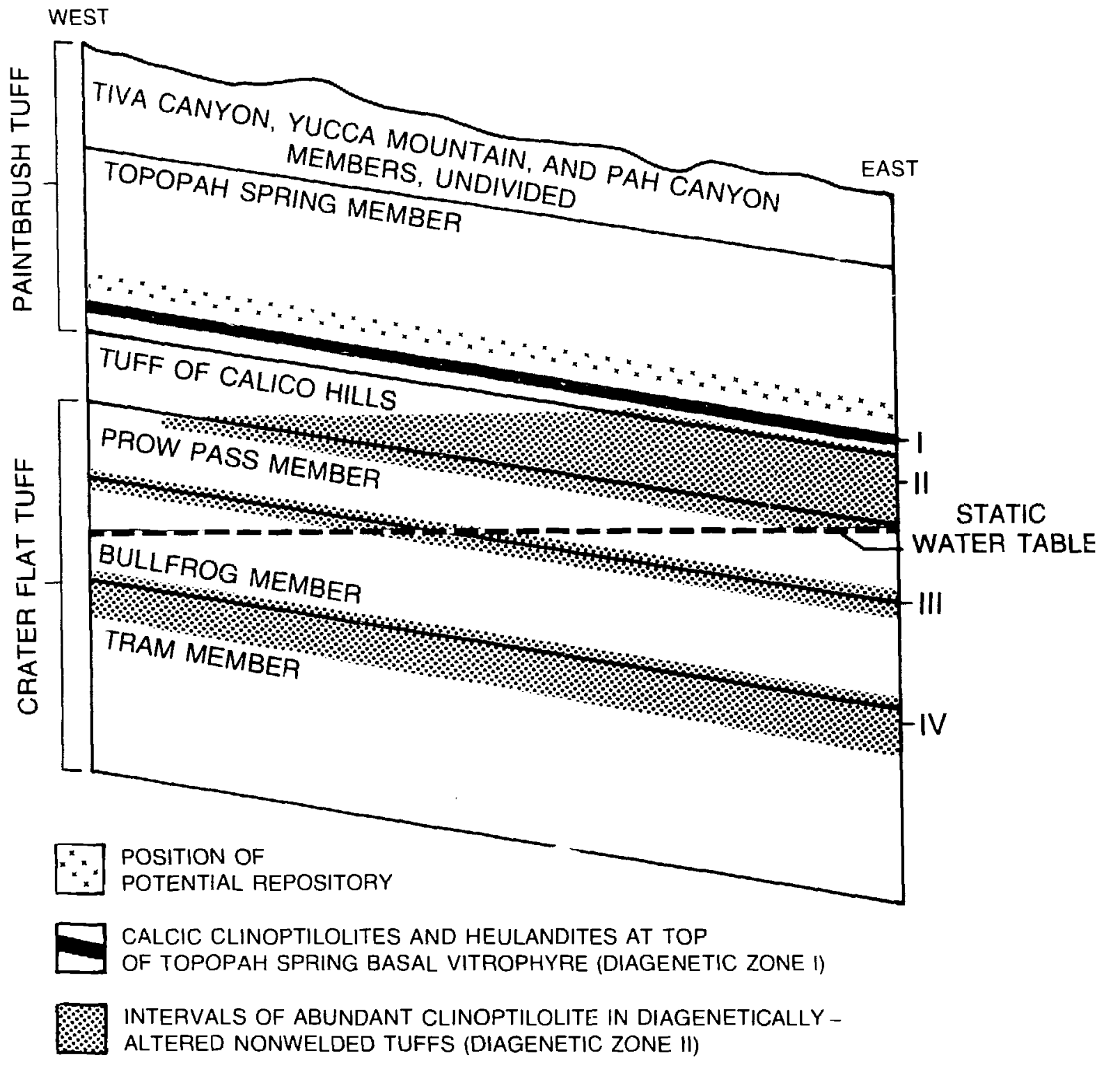

NUMBERS I THROUGH IV REFER TO ZEOLITIC - TUFF INTERVAL NCMENCLATURE OF VANIMAN et al (1984,TABLE III).

\section{Fig. 3.}

Schematic cross section of Yucca Mountain, Nevada, showing position of potential repository and principal zeolitic tuffs. 
This report describes the chemistry of diagenetically altered tuffs and their constituent authigenic minerals at Yucsa Mountain. The compositions of these tuffs vary considerably across the repository exploration block and along potential pathways to the accessible environment. Experimental studies show that physical properties of zeolites vary with composition (Bish 1984; 1985). Compositionally dependent zeolite properties important for repository performance assessment include expansion/contraction behavior, hydration/dehydration behavior, and ion exchange. Used in conjunction with experimental results, data presented here can be used to model themal and sorptive properties of tuffs in the repository environment.

\section{II . GEOLOGIC SETTING}

Yucca Mountain lies within the southwest Nevada volcanic field in the southern Great Basin. It is a north-trending, east-tilted, fault-bounded mountain range that is a remnant of a formerly extensive Miocene-Pliocene volcanic plateau centered around the Timber Mountain-0asis Valley caldera complex (Christiansen et al. 1965, 1977; Byers et al. 1976). The predominant lithologies at Yucca Mountain are high-silica rhyolitic ash-flow tuffs (Table I). However, two large-volume ash-flow cooling units in the upper part of the sequence, the Tiva Canyon and Topopah Spring Members of Paintbrush Tuff, are compositionally zoned and grade upward from rhyolite to quartz latite (Lipman et a1. 1966; Byers et al. 1976). Miñor lithologies at Yucca Mountain include bedded tuffs and intermediate to silicic lavas and breccias. The volcanic rocks in this region have an aggregate thickness of over $1.8 \mathrm{~km}$.

Diagenetic alteration is best developed in stratigraphic units that are nonwelded throughout or in the nonwelded tops and bottoms of ash-flow cooling units having densely welded, devitrified interiors. The nonwelded tuffs remained largely vitric after emplacement and were highly susceptible to alteration because of the instability of glass in the presence of groundwater. During diagenesis, glass was reolaced primarily by the zeolites clinoptilolite, heulandite, and mordenite. Analcime and authigenic feldspars replace clinoptilolite and mordenite in the deeper parts of the volcanic secuence. These authigenic minerals form four diagenetic mineral zones that become progressively less hydrous with depth. These mineralogic zones are similar tij those described by Iijima (1975, 1978, and 1980) for burial diagenesis of volcanic ash beds in thick sedimentary sequences. 
TABLE I

STRATIGRAPHY OF VOLCANIC UNITS AT YUCCA MOUNTAIN, NEVADA ${ }^{a}$

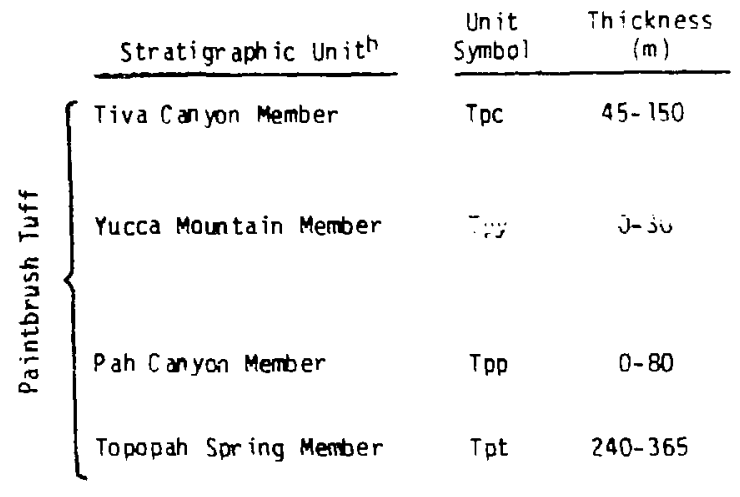

Tuff of Calico Hills Tht $35-290$

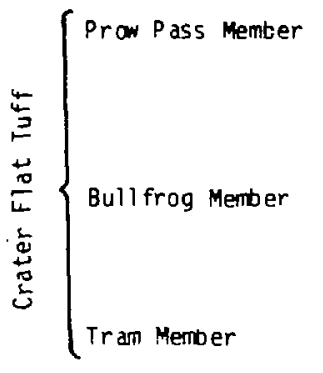

$\begin{array}{llr}\text { Dacite Flow Breccia } & \text { Tfb } & 0-120 \\ \text { Lithic Ridge Tuff } & \text { Tlr } & 185-305 \\ \text { Unnamed Older Tuffs } & \text { Tot } & 365+\end{array}$

\author{
Lithology
}

Ash-flow tuff: compound cooling unit; nonwelded vitric base: moderately to densely welded, devitrified interior with some vapor-phase crystallization.

Asin-i :. cuff: nonh-ided vitric top and base; partially welces devitrified interior with some vapor-phase crystallization; present under northern half of Yucca Mountain.

Ash-flow tuff; nonwelded and vitric throughout; present under northern haif of Yucca Mountain.

Ash-flow tuff: compositionally coned. compound cooling un it: nonwelded zones at top and base and moderately to densely welded, devitrified interior with zones of vapor-phase crystallization; vitrophyres at top and base of unit. Zealites occur both on top of basal vitrophyre and in nonwelded base of unit.

Ash-flow tuff: nonwelded to partially welded; thoroughly zeolitized at north end of exploration block; becomes vitric southward.

Ash-flow tuff: nonwelded zones at top and base: moderately welded, devitrified interior with mitior vapor-phase crystallization: nonwelded base is zeolitic; nonwelded top zeolitic in northern part of Yucca Mountain, but vitric to the south.

Ash-flow tuff: compound cooling unit: nonwel ded top and base, nonwelded to densely welded interior with thickness and occurrence of welding zones highly variable; zeolitic in nonwelded zones.

Ash-flow tuff; compound cooling unit; zones of partial to dense welding vary from drill hole to drill hole; zenlitic in its nonweided to partially welded parts: otherwise devitrified.

Flow breccia, lava, and tuffs; occurrence restricted to USW G-1; zeolites irregularly distributed throughout the unit.

Ash-flow tuff; nonwelded to moderately welded: devitrified, contains few zeolite horizons.

Ash-flow tuffs, lavas, reworked volcanic sediments: dacitic to rhyolitic compositions: contains few zeolite horizons. Includes units $a, b$, and $c$ of USW $G-1$.

\footnotetext{
Spengler et al. 1979, 1981; Maldonado and Koether, 1983; Scott and Castellanos, 1984.

bvolumetrically minor bedded tuffs (TO) between major stratiqraphic units not shown.
} 
Zone I, the shallowest zone, is characterized by tuffs that contain substantial unaltered volcanic glass. Smectite, opal, heulandite, and calcic clinoptilolite are minor alteration phases and concentrate in fractures and in thin discontinuous zones parallel to bedding. Two notable occurrences of zeolites in Zone I include (1) thin but widespread accumulations of smectites, calcic clinoptilolites, and heirandite at the top of the basal vitrophyre of the Topopah Spring Meinber and (2) a 130-m-thick sequence of smectite- and calcic clinoptilolite-rich tuffs in the Yucca Mountain, Pah Canyon, and lipper Topopah Spring Members in drill hole USW G-2. Despite their different stratigraphic and lithologic settings, both zeolite occurrences are similar in mineralogy and chemistry.

Zone II is characterized by complete replacement of volcanic giass by clinoptilolite \pm mordenite, with minor opal, quartz, potassium feldspar, and smectite. Clincptilolite is the most abundant zeolite in this zone and commonly makes up 50-75\% of tuffs. These zeolite-rich tuffs occur at well-defined intervals including (1) the interval extending from the base of the Topopah Spring Member, through the tuff of Calico Hills, and into the top of the Prow Pass Member; (2) the interval at the base of the Prow Pass Member and top of the Bullfrog Member; and (3) the intervai at the base of the Bull frog Member and top of the Tram Member. These intervals are equivalent to Zeolitic Intervals II, III, and IV, respectively, of Vaniman et al. 11984 , Table III) and are shown diagrammatically in Fig. 3. There are no significart mineralogic or chemical changes in authigenic minerals across stratigraphic contacts within these intervals, suggesting that lithology rather than stratigraphy controls sites of zeolitic alteration. Individual zeolitized beds range in thickness from 10 to $150 \mathrm{~m}$. These beds are major sorptive barriers for radionuclide migration because they occur along potential groundwater pathways below the repository in the unsaturated and saturated zones.

Diagenetic Zones II and IV are confined to the deeper structural levels of Yucca Mountain. Zone III is characterized by the progressive replacement of clinoptilolite and mordenite by analcime, potassium feldspar, quartz, and minor calcite and smectite. Zone IV, the deepest diagenetic zone penetrated by drill holes, is characterized by the replacement of analcime by authigenic albite. 


\section{METHODS}

A. Whole-Rock Compositions by X-Ray Fluorescence, Atomic Absorption, and Emmission Spectrophotometry

Whole-rock major-element analyses (Appendix A) were determined by $x$-ray fluorescence (XRF) for samiles from drill holes UE-25a\#1, UE-25b\#I(H), UE-25p\#1, USW G-2, USW G-3, USW GU-3, USW G-4, and J-13 (Fig. 1). Most samples are from continuously cored exploratory drill holes; samples from UE-25p\#1 are from bit cuttings that were collected at $3-m(10-f t)$ intervals. Two additional samples were collected from outcrop at Prow Pass, located at the northern end of Yucca Mountain (Fig. 1). Many of these samples were also analyzed for mineral contents by x-ray diffraction (Bish and Vaniman 1985).

Samples were prepared by powdering and homogenizing 15-20 $\mathrm{g}$ of material in a shatterbox. Duplicate $10 \mathrm{~g} g$ sample splits were dried to $1000^{\circ} \mathrm{C}$, for 4 hours, and then $1-g$ solits were fused with $9 \mathrm{~g}$ of lithium tetraborate flux. Drying at $1000^{\circ} \mathrm{C}$ destroys the zeolitic crystal structures and eliminates gross weighing errors introduced by rapid rehydration of zeolites upon cooling. Wotatile contents (orimarily water) were determined by differences in sample weights at room temperature and after heating at $1000^{\circ} \mathrm{C}$.

Major elements yere determined with an automated Rigaku wavelength dispersive XRF spectrophotometer. A library of $x$-ray intensities for rock standards was used to calculate elemental concentrations (Valentine 1983). A fundamental Darameters program made matrix corrections for $x$-ray absorption and secondary fluorescence.

Splits of 20 zeolitic tuff semples were also analyzed by John Husler of the University of New Mexico and Norman Suhr of Pennsylvania State University to determine the reliability of the XRF results and specifically to evaluate a problein of sodium loss introduced by the XRF sample preparation technique. Husler and Suhr determined silica gravimetrically and iron titrimetrically. Husler analyzed all other elements, including total iron, by atomic absorption (AA), and Suhr determined .71 other elements by plasma-source emission spectrophotometry (ES).

Comparison of XRF analyses with those determined by AA and ES (Appendix B) shows that the three analytical methods agree well except for sodium (Fig. 4). Elemental concentrations determined by the three laboratories at Los Alamos, Pennsylvania State University, and the University of New Mexico generally agree within $5 \%$ for major components and $20 \%$ for minor components. 

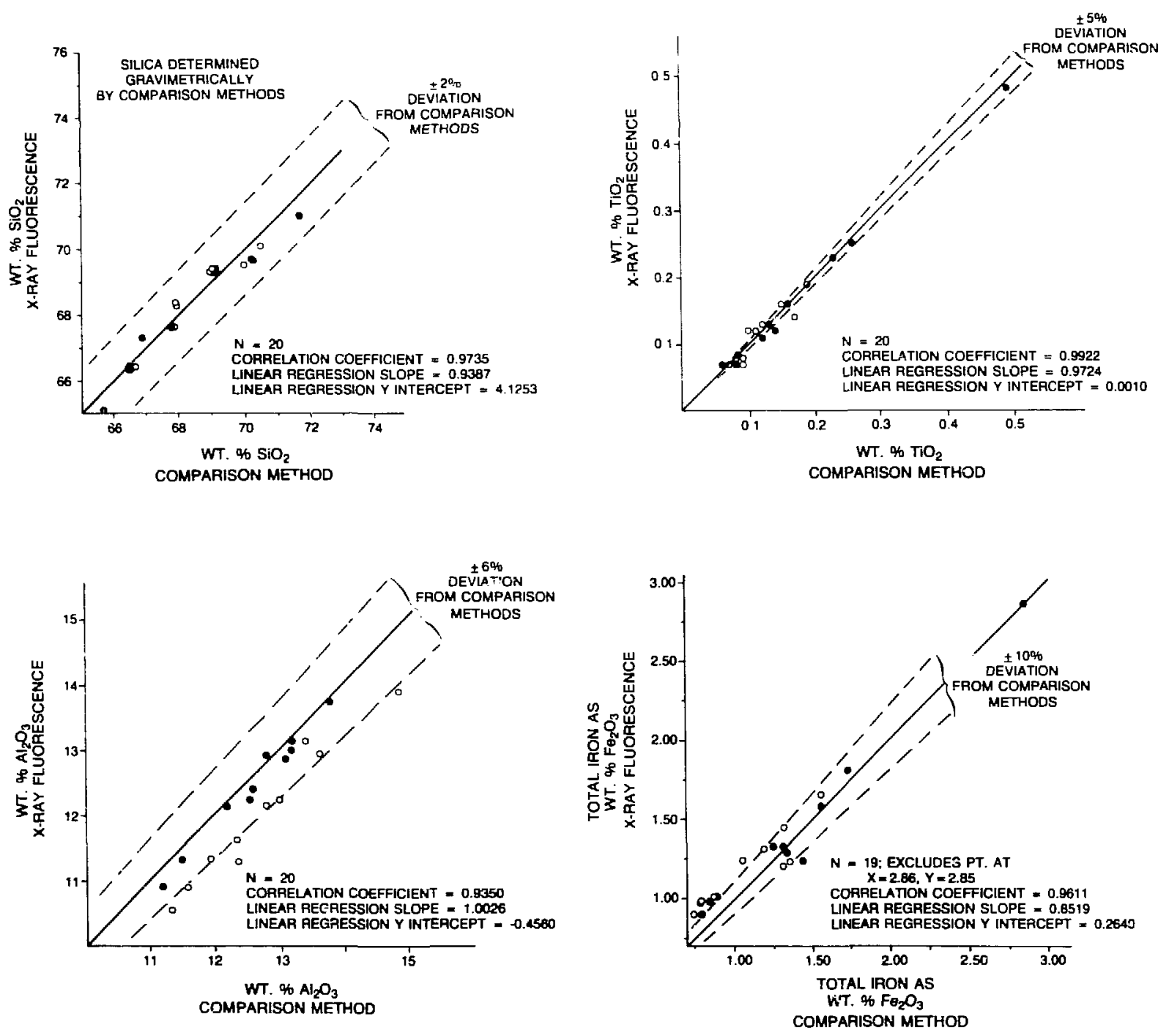

Fig. 4.

Comparison of whole-rock analyses determined by $x$-ray fluorescence vs. analyses by atomic absorption and emission soectrophotometry. Solid diagonal lines have a slope of one, indicating perfect correlations between analytical metnods. Dashed diagonal lines represent percent deviation of $x$-ray fluorescence analysis from atomic absorption and emission spectrophotometry analyses. 

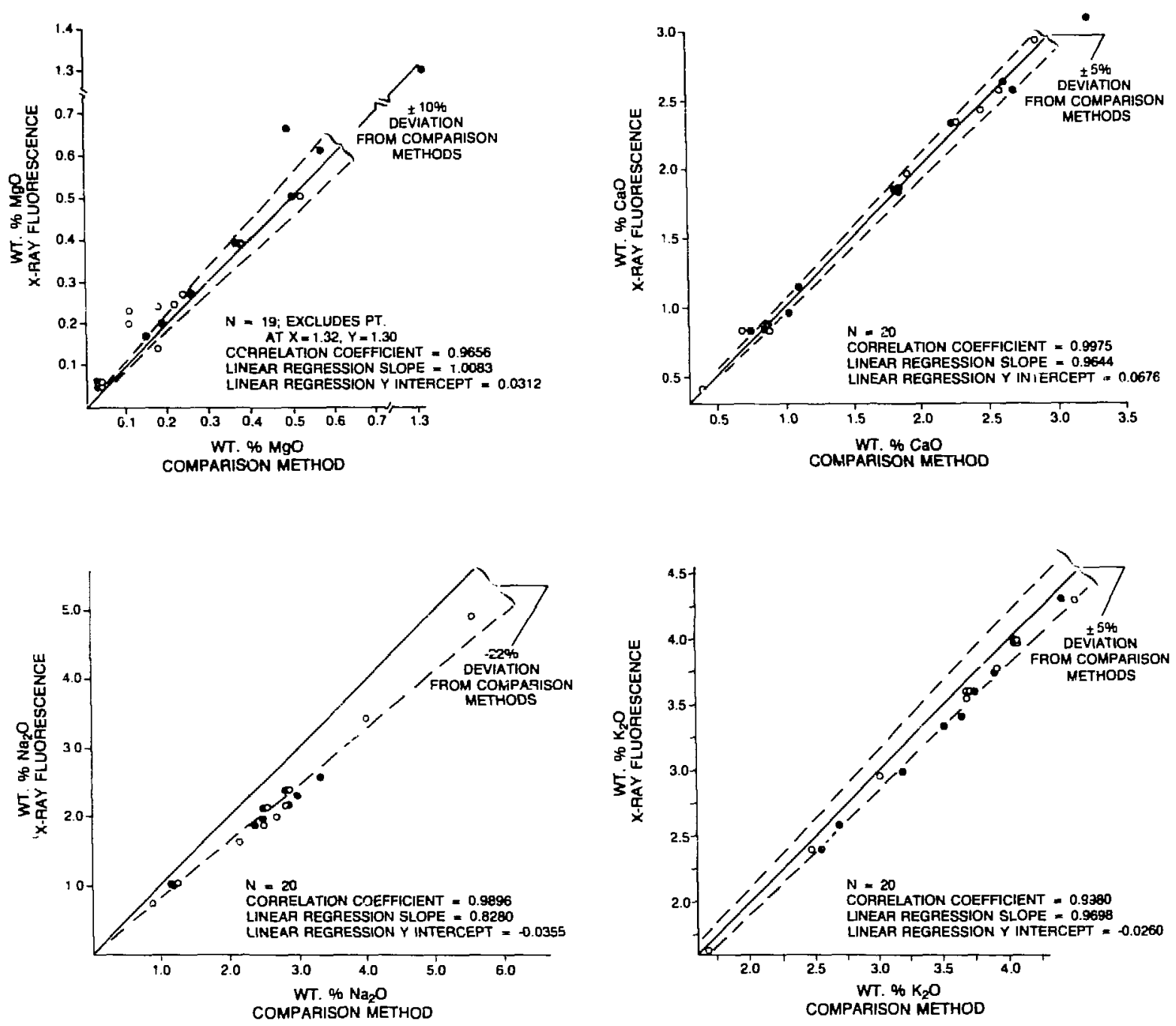

COMPARISON METHODS

O ATOMIC ABSORPTION SPECTROPHOTOMETRY (UNM)

- DC. PLASMa EMISSION SPECTROPHOTOMETRY (PENN STATE) 
However, XRF analyses of sodium are systematically $22 \%$ lower than sodium concentrations determined by $A A$ or ES. Because sodium concentrations can be accurately duplicated in well-characterized standards, we believe the systematically lower sodium concentrations in samoles analyzed by XRF result from partial loss of sodium along with water when the samples are dried at $1000^{\circ} \mathrm{C}$. As a result of these interlaboratory comparisons, sodium concentrations were redetermined in new samples by $A A$ and/or ES for all samples in Appendix A. Fotassium also appears to be systematically lower (2-5\%) in XkF analyses compared to AA or ES; however, this difference is relatively minor and the XRF analyses for $K_{2} 0$ are retained in Appendix $A$. Aluminum concentrations determined by XRF agree well witn those by ES. However, there is a 4-6\% systematic deviation in $A A$ aluminum concentrations compared to XRF and ES (Fig. 4); these differences are probably due to a calibration bias in the $A A$ analyses.

B. Whole-Rock Compositions by Automated Neutron Acivation Analysis

Whole-rock analyses for 32 major and trace elements were determined by automated neutron activation ancilyses (ANAA), according to the procedures of Minor et al. (198?). ANAA analyses were performed for 86 samples from drill hole USW G-1, 3 samples from USW G-2, 2 samoles from USW GU-3, and 3 samples from tuff of Calico Hills outcrops at Prow Pass in the northern part of Yucca Mountain (Appendix C). Most samoles were lightly ground in a steel mortar; those few sambles without cobalt values were powdered in a shatterbox, which introduces substantial (unreported) cobalt and tungsten contamination into the sample.

Approximately $4 \mathrm{~g}$ of air-dried material were analyzed for each sample. Analyses were performed under the same conditions as those described by Garcia et al. (1982) for NBS standards. Therefore, analytical uncertainties for values of each element in Appendix $C$ should be similar to the standard deviation for this element in the NBS standards (Appendix D), where elemental concentrations of the unknown and NBS standard match closely. Analytical uncertainties provided for certain elements and for select samples in Appendix $C$ are the result of either an additional count 6 months after irradiation or of longer irradiation and count times. Those uncertainties were calculated from count rates obtained from raw spectra, which were not available for most samoles. 
Volcanic glass, clinoptilolite, analcime, and authigenic feldspar compositions (Appendices $\mathrm{F}-\mathrm{H}$, respectively) were determined with an automated Cameca electron microprobe operated at $15 \mathrm{keV}$ and 15- to 20-nA beam currents. Calibration standards included feldspars for $\mathrm{Si}, \mathrm{Al}, \mathrm{Na}, \mathrm{K}, \mathrm{Ca}$, and $\mathrm{Ba}$, and amohibole and pyroxene for $\mathrm{Mg}, \mathrm{Fe}$, and $\mathrm{Ti}$. Wavelength dispersive $\mathrm{x}$-ray counts for each element were collected for 15 to 20 seconds or less if 10000 counts were acquired. Compositions are corrected for differential matrix effects using the methods of Bence and Albee (1968). Sodium was counted first during analysis because it tends to migrate from the region excited by the electron beam. Use of a square rastered electron beam with an edge of 15 to $25 \mathrm{um}$ minimized sodium migration, water loss, and structural decomposition of zeolites. When possible, the sample was moved beneath the electron beam to minitnize sodium loss during analysis.

Desoite the precautions taken, sodium loss remained a significant problem during the analysis of clinoptilolite. Figure 5 shows a progressive decline in sodiun! counts during a 50-second count. Calculated errors for a typical 15- to 20-second analysis range from 5 to $12 \%$ of the sodium present. Efforts to reduce sodium loss by lowering beam currents were unsatisfactory because poorer counting statistics for all elements outweighs the small improvement in retention of sodium. The structural formula of all zeolites, including clinoptilolite, requires $(A l+F e)=(N a+K+2 C a+2 M g)$ for electrical neutrality, assuming the small amount of $\mathrm{Fe}$ occurs as Fe(III). Zeolite analyses were considered acceptable if the ratio $(\mathrm{Al}+\mathrm{Fe}) /(\mathrm{Na}+\mathrm{K}+2 \mathrm{Ca}+2 \mathrm{Mg})$ was less than 1.15 . Ratios less than 1.0 are uncommon because of problems with sodium loss. Analcime does not lose sodium when analyzed under the conditions used in this study (Fig. 5).

Mordenite has been identified in many altered tuffs at Yucca Mountain by $x$-ray diffraction (Bish and Vaniman 1985), but its chemistry has not been studied. Individual mordenite crystals are so fine grained and intergrown with other authigenic phases that analysis by electron microbrobe is not feasible. Determination of mordenite compositions by whole-rock methods are not feasible because monomineralic beds of mordenite do not occur at Yucca Molintain.

Glass and mineral compositions in Appendices $E$ through $F$ include new unpublished data as well as analyses compiled from previously published drill 


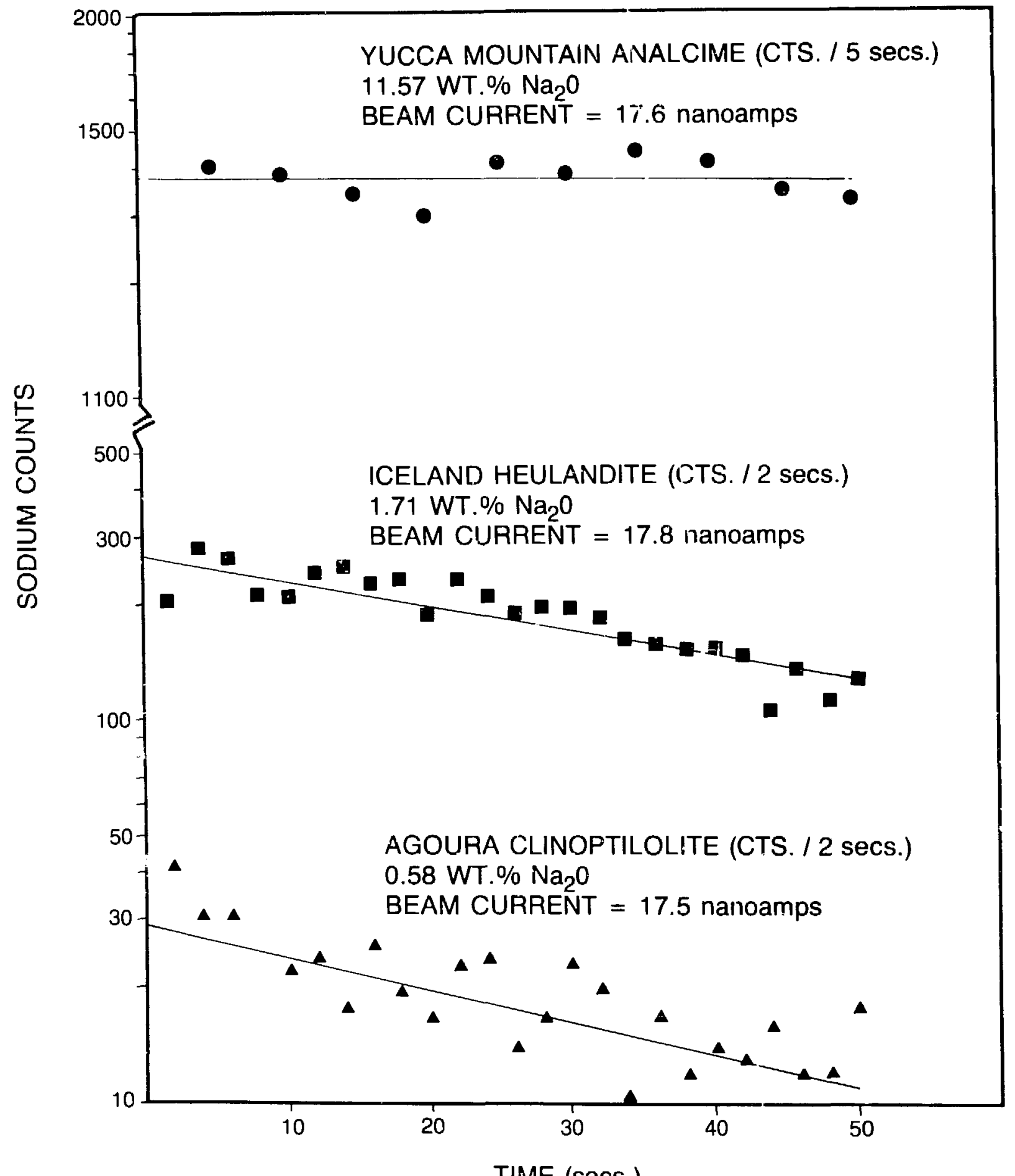

TIME (secs.)

Fig. 5 .

Semilogarithmic plot showing sodium count rates as a function of time for a variety of zeolites. Analyses were performed using a stationary electron microprobe beam with an accelerating potential of $15 \mathrm{keV}$. The beam was rastered with a $20-\mu \mathrm{m}$ edge. 
hole reports (Sykes et al. 1979; Heiken and Bevier 1979; Caporuscio et al. 1982; Broxton et al. 1982; Vaniman et al. 1.984; Levy 1984; and Cadoruscio et al. 1985). Data from these previous reports were not included if $(\mathrm{A}]+\mathrm{Fe}) /(\mathrm{Na}+\mathrm{K}+2 \mathrm{Ca}+2 \mathrm{Mg})$ was greater than 1.15 for zeolites or if other problems with mineral stoichiometry were suspected. In some cases secondary minerals were re-analyzed because earlier data sets included analyses of mixed mineral phases along with analyses of single minerals.

\section{RESULTS}

All analyses in the appendices are grouped by drill hole, wi⿳⺈ individual analyses for each drill hole arranged by increasing depth. Table II compares representative zeolitic and unaltered tuff compositions for each of the principal stratigraphic units of Fig. 3. Table II also provides a comparison of zeolitic tuff compositions on the eastern side of Yucca Mountain (calcic suite) with those occurring on the west (alkalic suite). Table III compares representative clinoptilolite compositions from the eastern and western side of Yucra Meuntain.

Whole-Rock Compositions: Whole-rock compositions for volcanic rocks of Yucca Mountain are presented in Appendices A through C. Additional whole-rock compositions for tuffs in drill hole USW G-1 are published in Zielinski (1983; Table 2) and Spengler et a). (1981; Table 5). Scott and Castellanos (1984; Table 5) present whole-rock compositions for seiected samples from USW G-1, USW G-2, USW GU-3/G-3, and for outcrop samples.

kelative chemical changes associated with diagenesis are evaluated by comparing unaltered and altered tuff compositions on a constant-aluminum basis. This method assumes that aluminum is relatively insoluble and does not migrate significantly during diagenesis (Hay 1963). The low aluminum contents for groundwaters in volcanic rocks of the arid southwest suggest this assumption is valid (Lipman 1965). Figure 6 shows the logarithmic ratio of major elements in zeolitic tuffs to those in unaltered tuffs, normalized to the same aluminum content, for a representative suite of samples (Table II). Devitrified and vitric tuffs were used to represent unaltered tuff compositions.

Alkalies and alkaline earths were the elements most strongly affected in zeolitic tuffs by diagenetic alteration (Fig. 6). Sodium, potassium, calcium, and magnesium contents of zeolitic tuff deviate from those of unaltered tuff 


\section{TABLE II}

REPRESENTATIVE BULK ROCK COMPOSITIONS FOR TUFFS AT YUCCA MOUNTAIN, NEVADAa

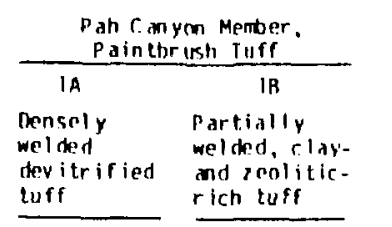

Tompah Sp-ing Member.

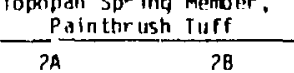

\begin{tabular}{|c|c|c|}
\hline $3 A$ & 38 & $3 r$ \\
\hline $\begin{array}{l}\text { Nonwelifed } \\
\text { vitric } \\
\text { tuff }\end{array}$ & $\begin{array}{l}\text { Nonwelded } \\
\text { zenlitic tuff. } \\
\text { alkalic ssitar }\end{array}$ & $\begin{array}{l}\text { Nonwelded } \\
\text { reolitic tuff, } \\
\text { ralcic sulite }\end{array}$ \\
\hline
\end{tabular}

Dlagenetic

\section{I}

Somple

67F B-3E

6. - 675

Inal tered

lower

vitrophye

\begin{tabular}{l} 
Altered \\
:awer \\
vitrophyre \\
\hline
\end{tabular}

tuff

alkalis suit

ralcic suite

\begin{tabular}{|c|c|}
\hline $\mathrm{SiO}_{2}$ & 72.9 \\
\hline $\mathrm{TiO}_{2}$ & 0.28 \\
\hline $\mathrm{Al}_{2} \mathrm{O}_{3}$ & 13.9 \\
\hline $\mathrm{Fe}_{2} \mathrm{O}_{3}$ & 1.4 \\
\hline Fé & 0.04 \\
\hline $\operatorname{mo} 0$ & 0.06 \\
\hline $\mathrm{MgO}$ & 0.33 \\
\hline$C .+0$ & 0.92 \\
\hline $\mathrm{Na}_{2} \mathrm{O}$ & 3.8 \\
\hline$k_{2} 0$ & 5.1 \\
\hline$P_{7} 0_{5}$ & 0.02 \\
\hline $\operatorname{Lot}^{d}$ & 0.97 \\
\hline
\end{tabular}

\section{3}

0.37

16.8

$1.83^{c}$

$25 \mathrm{Al} \cdot 12 \mathrm{BO}$

I

11

11

GA- $13 \mathrm{IM}$

[รป]. - M9E.

64- 1544C.

$25,131-1323$

7. 0

7n.?

0.14

74.5

69.1

60.1

0.10
$1 ? .4$

16.7

$1.44^{\mathrm{C}}$

11.1

0.06

11.5

$1.07^{\mathrm{C}}$

$1.10^{c}$

0.81

$$
0.04
$$

0.09

0.90

0.31

0.66

3.40

3.51

1.90

0.72

0.02

0.01

$\frac{3.79}{99.8}$

$\frac{5.91}{101.5}$

0.08

$$
0.12
$$

0.17

3.20

4. 34

0.01

$\frac{3.58}{99.5}$

0.03

0.03

0.75

?.8?

4.10

0.00

0.11

13.4

$1.13^{\mathrm{C}}$

0.05

0.94

$3.3 ?$

1.23

$? .64$

0.01

10. 8.90

\begin{tabular}{|c|c|c|c|c|c|c|c|}
\hline $\begin{array}{l}S i \\
I i \\
\text { Al }\end{array}$ & $\begin{array}{c}68.4 \\
0.20 \\
15.4\end{array}$ & $\begin{array}{c}66.0 \\
0.25 \\
20.3\end{array}$ & $\begin{array}{c}72.2 \\
0.07 \\
14.3\end{array}$ & $\begin{array}{c}69.6 \\
0.10 \\
19.5\end{array}$ & $\begin{array}{c}73.0 \\
0.07 \\
13.5\end{array}$ & $\begin{array}{c}72.8 \\
0.04 \\
14.3\end{array}$ & $\begin{array}{c}71.6 \\
0.09 \\
16.3\end{array}$ \\
\hline $\begin{array}{l}\mathrm{Fe}^{+3} \\
\mathrm{Fe}^{+2}\end{array}$ & $\begin{array}{l}0.98 \\
0.03\end{array}$ & $1.41^{\mathrm{C}}$ & $0.78^{\mathrm{C}}$ & $1.07^{\mathrm{C}}$ & $0.81^{C}$ & $\begin{array}{l}0.64 \\
0.03\end{array}$ & $0.88^{c}$ \\
\hline $\begin{array}{l}m \\
M g \\
C . g \\
M a \\
K \\
p\end{array}$ & $\begin{array}{l}0.04 \\
0.46 \\
0.93 \\
6.93 \\
6.68 \\
0.02\end{array}$ & $\begin{array}{l}0.12 \\
2.71 \\
2.86 \\
7.74 \\
3.56 \\
0.04\end{array}$ & $\begin{array}{l}0.05 \\
0.45 \\
0.69 \\
6.43 \\
4.98 \\
0.01\end{array}$ & $\begin{array}{l}0.06 \\
1.33 \\
3.73 \\
3.65 \\
0.91 \\
0.02\end{array}$ & $\begin{array}{l}0.05 \\
0.17 \\
0.81 \\
6.08 \\
5.43 \\
0.01\end{array}$ & $\begin{array}{l}0.02 \\
0.05 \\
0.86 \\
5.76 \\
.51 \\
0.00\end{array}$ & $\begin{array}{l}0.04 \\
1.45 \\
3.58 \\
2.47 \\
3.49 \\
0.01\end{array}$ \\
\hline
\end{tabular}

\section{Element Percent}

Analyses by combination of $x-r$ ay fluorescence and atomic absorption and plasma emission spectrophotometry. Analysis of sample 67F8-JE from Ouinlivan and Byers (1971) and 61-2557 from Ziel insk i (1933).

${ }^{C}$ Total $\mathrm{Fe}$ as $\mathrm{Fe}_{2} \mathrm{O}_{3}$.

doos on ignition, decrease in sample weint at heating at $1000^{\circ} \mathrm{C}$ for $4 \mathrm{~h}$. Lor for G2-675 not measured but doss on ignition, decrease in sanple weipht at heating
determined by difference in analytical total from look. 
TABLE II (cont)

\begin{tabular}{|c|c|c|c|c|c|c|c|c|c|}
\hline & \multicolumn{3}{|c|}{$\begin{array}{l}\text { Prow Pass Member, } \\
\text { Cirater Flat Tuff }\end{array}$} & \multicolumn{3}{|c|}{$\begin{array}{l}\text { Bullfrog Member, } \\
\text { Crater Flat Tuff }\end{array}$} & \multicolumn{3}{|c|}{$\begin{array}{l}\text { Tram Menber, } \\
\text { Crater Fl.t. Tuff }\end{array}$} \\
\hline & $A A$ & 48 & $4 C$ & $5 A$ & $5 B$ & $5 C$ & $6 A$ & $6 B$ & $6 C$ \\
\hline & $\begin{array}{l}\text { Nonwel ded } \\
\text { vitric } \\
\text { tuff }\end{array}$ & $\begin{array}{l}\text { Nonwelded } \\
\text { zeolitic tuff, } \\
\text { alkalic suite }\end{array}$ & $\begin{array}{l}\text { Nonwelded } \\
\text { zealitic tuff, } \\
\text { calcic suite }\end{array}$ & $\begin{array}{l}\text { Partially } \\
\text { welded } \\
\text { devitrified } \\
\text { tuff }\end{array}$ & $\begin{array}{l}\text { Noriwelded } \\
\text { zeolitic tuff, } \\
\text { dika'ic suite }\end{array}$ & $\begin{array}{l}\text { Nonwelded } \\
\text { zeolitic tuff, } \\
\text { calcic suite }\end{array}$ & $\begin{array}{l}\text { Partially } \\
\text { welded } \\
\text { devitrified } \\
\text { tuff }\end{array}$ & $\begin{array}{l}\text { Nonwelded } \\
\text { zeolitic tuff. } \\
\text { alkaic suite }\end{array}$ & $\begin{array}{l}\text { Nonwelded } \\
\text { zeolitic tuff, } \\
\text { calcic suite }\end{array}$ \\
\hline \multicolumn{2}{|c|}{$\begin{array}{l}\text { Diagenet ic } \\
\text { Zone }\end{array}$} & 11 & II & & 11 & II & & II & 11 \\
\hline $\begin{array}{l}\text { Sample } \\
\text { No. }\end{array}$ & GU3- 1598 & GU3-1986B & G4-2131C & $.113-2133$ & G $1-2557$ & $2581 H-2879 C$ & $.113-2952$ & G3-3589C & G4-2792B \\
\hline $\mathrm{SiO}_{2}$ & 73.1 & 67.9 & 65.7 & 75.3 & 69.0 & 66.9 & 74.8 & 66.5 & 67.9 \\
\hline $\mathrm{TiO}_{2}$ & 0.11 & 0.10 & 0.12 & 0.11 & 0.11 & 0.26 & 0.16 & 0.49 & 0.15 \\
\hline $\mathrm{Al}_{2} \mathrm{O}_{3}$ & 12.2 & 12.4 & 13.2 & 12.6 & 12.4 & $13 . ?$ & 17.2 & 13.8 & 12.3 \\
\hline $\mathrm{Fe}_{2} \mathrm{O}_{3}$ & \multirow{2}{*}{$1.41^{\mathrm{C}}$} & 1.20 & 1.12 & \multirow{2}{*}{$1.44^{\mathrm{C}}$} & \multirow{2}{*}{$1.51^{\mathrm{C}}$} & 1.48 & \multirow{2}{*}{$1.41^{\mathrm{C}}$} & 2.59 & 1.04 \\
\hline $\mathrm{FeO}$ & & 0.03 & 0.12 & & & 0.24 & & 0.26 & 0.13 \\
\hline $\mathrm{MnO}$ & 0.10 & 0.07 & 0.02 & 0.09 & 0.05 & 0.04 & 0.05 & 0.06 & 0.03 \\
\hline Mgo & 0.20 & 0.11 & 0.15 & 0.23 & 0.22 & 1.32 & 0.21 & 0.57 & 0.12 \\
\hline $\mathrm{Ca} 0$ & 0.83 & 0.87 & 2.53 & 0.75 & 1.33 & 3.74 & 0.89 & 1.82 & 2.93 \\
\hline $\mathrm{Na}_{2} \mathrm{O}$ & 3.29 & 3.44 & 2.46 & 4.02 & 3.50 & 1.18 & 3.70 & $? .98$ & 2.68 \\
\hline $\mathrm{K}_{2} \mathrm{O}$ & 4.37 & 3.72 & $3.5 ?$ & 4.38 & 3.05 & 3.90 & 4.46 & 3.65 & 1.68 \\
\hline $\mathrm{P}_{2} \mathrm{O}_{5}$ & $0.0 ?$ & 0.02 & 0.00 & 0.03 & 0.00 & 0.00 & 0.02 & 0.00 & 0.03 \\
\hline$L O I^{d}$ & 3.41 & 10.2 & 11.1 & 0.30 & 7.88 & 7.86 & 0.94 & 7.23 & 10.9 \\
\hline \multirow[t]{2}{*}{ Total } & 99.0 & 100.1 & 100.1 & 99.3 & 99.1 & 99.7 & 98.8 & 100.0 & 100.1 \\
\hline & \multicolumn{6}{|c|}{ Element Percent } & & & \\
\hline Si & 71.7 & 70.7 & 69.4 & 71.1 & 70.8 & 69.2 & 71.5 & 67.4 & 72.0 \\
\hline $\mathrm{Ti}$ & 0.08 & 0.08 & 0.09 & 0.08 & 0.08 & 0.20 & 0.11 & 0.37 & 0.12 \\
\hline & 14.1 & 15.2 & 16.4 & 14.0 & 15.0 & 16.1 & 13.8 & 16.5 & 15.4 \\
\hline $\mathrm{Fe}^{+3}$ & $1.04^{c}$ & 0.94 & 0.89 & $1.02^{c}$ & $1.17^{c}$ & 1.15 & $1.01^{C}$ & 1.98 & 0.83 \\
\hline $\begin{array}{l}\mathrm{Fe}^{+2} \\
\mathrm{Mn}\end{array}$ & 0.07 & $\begin{array}{l}0.03 \\
0.05\end{array}$ & $\begin{array}{l}1.11 \\
0.01\end{array}$ & 0.05 & 0.03 & $\begin{array}{l}0.21 \\
0.03\end{array}$ & 0.03 & $\begin{array}{l}0.22 \\
0.04\end{array}$ & $\begin{array}{l}0.11 \\
0.02\end{array}$ \\
\hline $\mathrm{Mg}$ & 0.29 & 0.17 & 0.24 & 0.32 & 0.34 & $? .03$ & 0.30 & 0.86 & 0.28 \\
\hline Cá & 0.87 & 0.97 & 2.98 & 0.76 & 1.46 & 3.59 & 0.91 & 1.98 & 3.33 \\
\hline $\mathrm{Na}$ & 6.26 & 6.94 & 5.04 & 7.36 & 7.16 & 2.37 & 6.86 & 5.86 & 5.51 \\
\hline$k$ & 5.47 & 4.94 & 4.75 & 5.27 & 3.99 & 5.15 & 5.44 & 4.72 & 2.27 \\
\hline 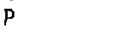 & 0.02 & 0.02 & 0.00 & 0.02 & 0.00 & 0.00 & 0.02 & 0.00 & 0.03 \\
\hline
\end{tabular}


TABLE III

REPRESENTATIVE ELECTRON MICROPROBE ANALI'SES OF CLINOPTILOLITE, YUCCA MOUNTAIN, NEVADA

Dingenetic zone 1

\begin{tabular}{|c|c|c|}
\hline Rnck Un it & $\begin{array}{l}\text { Path Con wn } \\
\text { Mentiter }\end{array}$ & $\begin{array}{l}\text { Topnpah } \\
\text { Spr in n. } \\
\text { Mether }\end{array}$ \\
\hline Samplp No. & C., $-5,5 M$ & H5. $166 \mathrm{~B}^{\circ}$ \\
\hline $\mathrm{SiO}_{2}$ & 65.5 & 65.5 \\
\hline $\mathrm{TiO}_{?}$ & 10.103 & $0.0 ?$ \\
\hline $\mathrm{Al}_{3} \mathrm{n}_{3}$ & 17.8 & 13.1 \\
\hline $\mathrm{Fe}_{2} \mathrm{O}_{3}$ & $0 . n 0$ & $0.0 n$ \\
\hline MqO & 1.47 & 0.86 \\
\hline C. a0 & 4.71 & 5.19 \\
\hline BaO & $0.0 ?$ & 0.00 \\
\hline $\mathrm{Ma}_{2} \mathrm{O}$ & $0.1 \mathrm{~A}$ & 0.17 \\
\hline$k_{2} 0$ & 1.09 & $0 . ? 1$ \\
\hline Total & ค6. 3 & $85 . ?$ \\
\hline
\end{tabular}

\begin{tabular}{|c|c|c|c|c|c|c|c|c|}
\hline \multirow{2}{*}{$\begin{array}{l}\text { CALCIS S } \\
\text { rulf of } \\
\text { ralico } \\
\text { Hille }\end{array}$} & \multicolumn{3}{|c|}{ SUITE, EASTERH YUITA MOUNIAIN } & \multicolumn{5}{|c|}{ ALKALIC SUITE, HESTRRN YIICCA MOUHTAIM } \\
\hline & $\begin{array}{l}\text { Prow Fass } \\
\text { Memtier }\end{array}$ & $\begin{array}{l}\text { Rullfrog } \\
\text { Menter }\end{array}$ & $\begin{array}{l}\text { Tuff of } \\
\text { Lithic } \\
\text { Ridim }\end{array}$ & $\begin{array}{l}\text { Tuff of } \\
\text { Calico } \\
\text { Hills }\end{array}$ & $\begin{array}{l}\text { Prom Pass } \\
\text { Member }\end{array}$ & $\begin{array}{l}\text { Pullifrng } \\
\text { Momtier }\end{array}$ & $\begin{array}{l}\text { Tram } \\
\text { Mentuer }\end{array}$ & $\begin{array}{l}\text { luff of } \\
\text { lith ir } \\
\text { Ridae }\end{array}$ \\
\hline $25 p 1-1250$ & $2501-1700$ & $25 a 1-2879$ & $2501-3330$ & $61.17 / 4$ & 6.3-1874 & $63-2615$ & 6.7-3589 & 6.3.4423 \\
\hline 6月. .6 & 67.1 & 56.1 & 60.6 & 611.1 & 68.2 & 6.3 .9 & 65.1 & 65.3 \\
\hline $0 . m$ & 0.00 & 0.00 & 0.00 & 0.00 & 0.00 & 0.00 & $0 . \mathrm{mo}$ & 0.00 \\
\hline 17.4 & 12.9 & 16.9 & 16.0 & 17.2 & 11.9 & 11.7 & 11.4 & 12.6 \\
\hline 0.00 & 0.00 & 0.05 & 0.00 & 0.00 & $0.0 m$ & 0.00 & 0.51 & 0.13 \\
\hline $0 . n 7$ & $0.3 ?$ & 0.22 & 0.08 & 0.09 & 0.70 & 0.00 & 0.17 & $0.7 ?$ \\
\hline 3.59 & 4.37 & 6.77 & $7.2 ?$ & 1.11 & 1.95 & 1.57 & 0.79 & 2.35 \\
\hline$n . m$ & 0.05 & 0.21 & 0.19 & 0.03 & 0.00 & 0.00 & 0.19 & 0.71 \\
\hline 1.13 & 1.55 & 0.89 & 0.85 & $? . P A$ & 1.69 & 2.RR & 2.23 & 3.21 \\
\hline 3.01 & 1.73 & 1.43 & 0.41 & 1.20 & 5.70 & 7.71 & 5.55 & 1.15 \\
\hline ดค . . & 8) .5 & A2.6 & 85.4 & ค月. 5 & 9H .7 & 82.7 & 85.9 & $85 . ?$ \\
\hline
\end{tabular}

$\begin{array}{lll}\mathrm{Si} & 28.9 & 29.2 \\ \mathrm{HI} & 0.00 & 0.01 \\ \mathrm{Al} & 7.17 & 6.98 \\ \mathrm{Fe}^{\mathrm{H}} & 0.00 & 0.00 \\ \mathrm{Mg} & 0.97 & 0.57 \\ \mathrm{Cd} & 7.00 & 7.48 \\ \mathrm{Ba} & 0.00 & 0.00 \\ \mathrm{Nd} & 0.15 & 0.15 \\ \mathrm{~K} & 0.61 & 0.12\end{array}$

$29.7 \quad \frac{\text { Unit-rell composition Based on } 72 \text { (0) }}{29.4} 27.5$

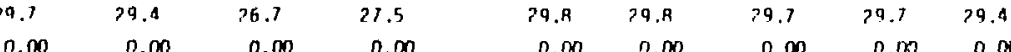

$\begin{array}{lllllllll}6 . .77 & 6.67 & 9.44 & 8.59 & 6.28 & 6.14 & 5.47 & 6.17 & 6.70\end{array}$

$\begin{array}{lllllllll}0.00 & 0.00 & 0.0 ? & 0.00 & 0.00 & 0.00 & 0.00 & 0.11 & 0.04\end{array}$

$\begin{array}{lllllllll}0.04 & 0.71 & 0.16 & 0.05 & 0.06 & 0.00 & 0.00 & 0.08 & 0.15\end{array}$

$\begin{array}{lllllllll}1.67 & 2.05 & 3.44 & 3.51 & 0.52 & 0.91 & 0.78 & 0.39 & 1.13\end{array}$

$\begin{array}{lllllllll}0.00 & 0.01 & 0.04 & 0.03 & 0.01 & 0.00 & 0.00 & 0.03 & 0.05\end{array}$

$\begin{array}{lllllllll}0.95 & 1.3 ? & 0.82 & 0.75 & 7.41 & 1.43 & 2.60 & 1.97 & ? .80\end{array}$

I. .65

0.69

O.B7

0.24

$2.35 \quad 2.90$

$\begin{array}{lll}2.60 & 1.97 & 7.80 \\ 1.61 & 3.23 & 0.66\end{array}$

$1.01 \quad 1.10$

1.05

$1.02 \frac{(\mathrm{A} 1+\mathrm{Fe}) /(2 \mathrm{Mg}+2 \mathrm{Co}+2 \mathrm{Ba}+\mathrm{Ma}+\mathrm{K})}{1.05}$

4. โ8 $4.41 \quad \frac{S i /(A)+F e)}{2.82}$

$1.06 \quad 1.00$

$1.11 \quad 0.99 \quad 1.09$

4.03

4. 18

$\frac{\text { Molx Exchongeable Cations }}{16}$

$$
16
$$

16
15

5

Ca+mg

Bo 92

$2 \%$

53

69

78

$4.75 \quad 4.85$

4.62

$4.77 \quad 4.36$

- Sample H5-1665 from Levy (1984o) and samples 63-2615. G3-3589, and G3-4423 from Van than et 31 . (1984).

Total Iron calculated as $\mathrm{Fe}_{2} \mathrm{O}_{3}$. 


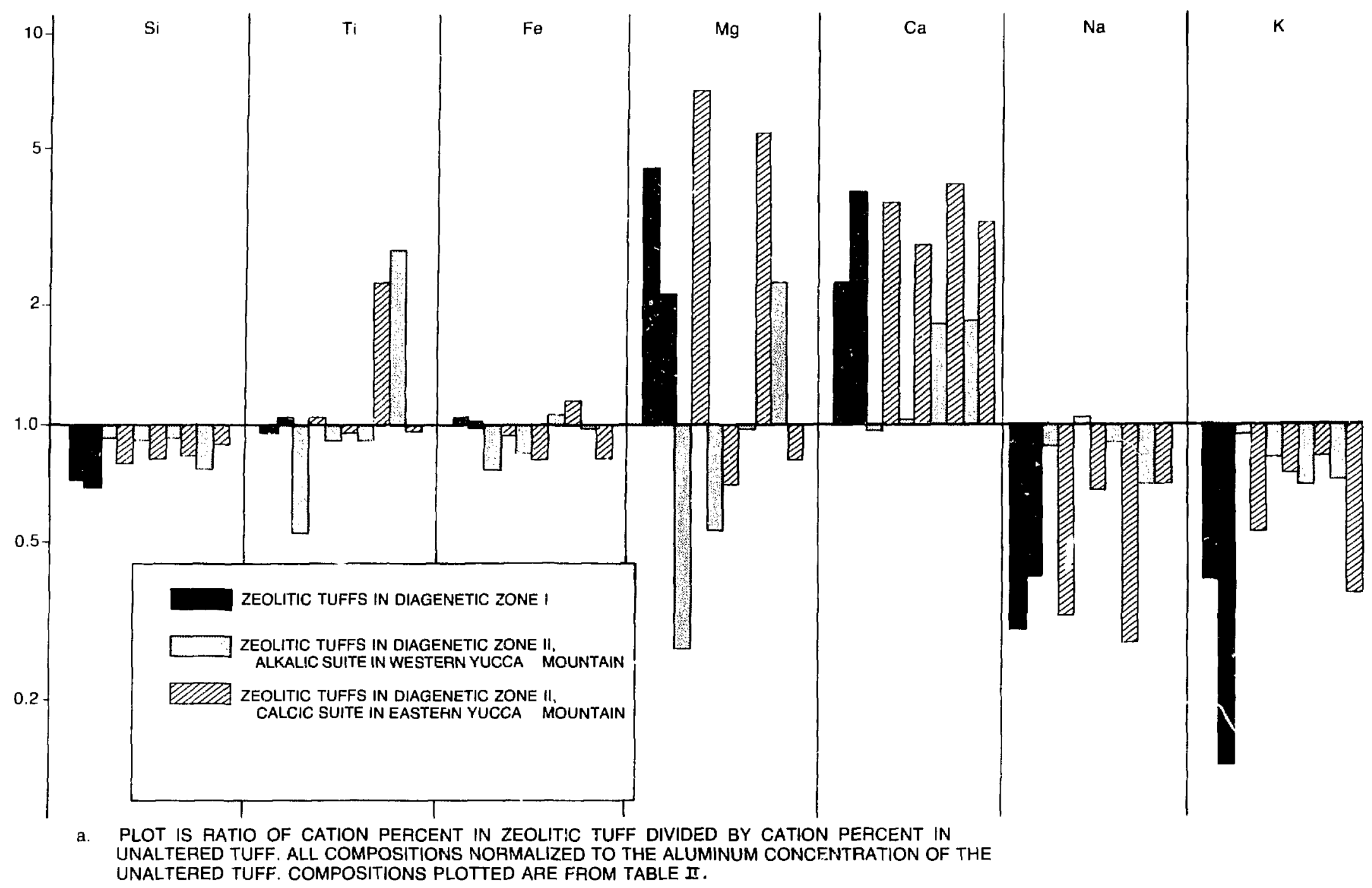

Fig. 6 .

Logarithmic plot showing elemental ratios, normalized to equal aluminum contents, for zeolitic and unaltered tuffs, Yucca Mountain, Nevada. 
by as much as a facter of 10. Sodium and potassium are generally strongly depleted in the zeolitic tuffs, whereas calcium is strongly enriched. Magnesium is strongly enriched in diagenetic zone I but is highly variable in Zone II. These elemental enrichments and depletions in alkalies and alkaline earths vary in detail and depend on the location of the tuff within Yucca Mountain. Silicon is systematically depleted in zeolitic tuffs, whereas iron and titanium show less systematic compositional changes. Iron in the zeolitic tuffs is strongly oxidized, with $90-100 \%$ of the iron in the (III) oxidation state in most zeolitic samples (Appendix B).

Triangular plots of whole-rock alkalies and alkaline earths (Fig. 7) show that the zeolitic tuff compositions change systematically across Yucca Mountain. These whole-rock chemical variations are mirrored in clinopti?o1 ites that occur in these tuffs.

Based upon mode of occurrence and compositional characteristics, zeolitic tuffs at Yucca Mountain can be divided into three compositional groups:

(1) calcium- and magnesium-rich tuffs associated with relatively thin zones of alteration in diagenetic Zone I;

(2) zeolitic tuffs of extremely variable composition in thick zones of alteration in diagenetic Zones II, III, and IV beneath the exploration block; and

(3) highly potassic zeolitic tuffs in zone II at the northern end of Yucca Mnintain.

Three whole-rock samples from the caicium- and magnesium-rich tuffs in Zone I were analyzed by a combination of XRF and ANAA techniques. Two of these samples (sample \#26, Appendix A; depth $1286 \mathrm{ft}$ in USW G-1, Appendix C) were collected from altered zone at the top of the basal vitrophyre of the Topopah Spring Member. The other sample (sample \#1, Appendix A) was collected from a smectite- and zeolite-rich tuff in the Pah Canyon Member in USW G-2. Ali three samples are characterized by $>2.84 \mathrm{wt} \% \mathrm{CaO}$ and $\geq 0.94 \mathrm{wt} \% \mathrm{MgO}$ when normalized to volatile-free compositions. Unaitered Topopah Spring and Pah Canyon tuffs tydically contain less than $0.65 \mathrm{wt} \% \mathrm{CaO}$ and $0.30 \mathrm{wt} \%$ MgO (Table II). Trace-element abundances in the altered tuffs are little affected by diagenetic alteration except for strontium, rubidium, and uranium. Strontium is concentrated in the zeolitic tuffs by a factor of 5 or more compared with that in unaltered tuff compositions. Rubidium and uranium are both depleted by a factor of 2 in the zeolitic tuffs (Table IV). 


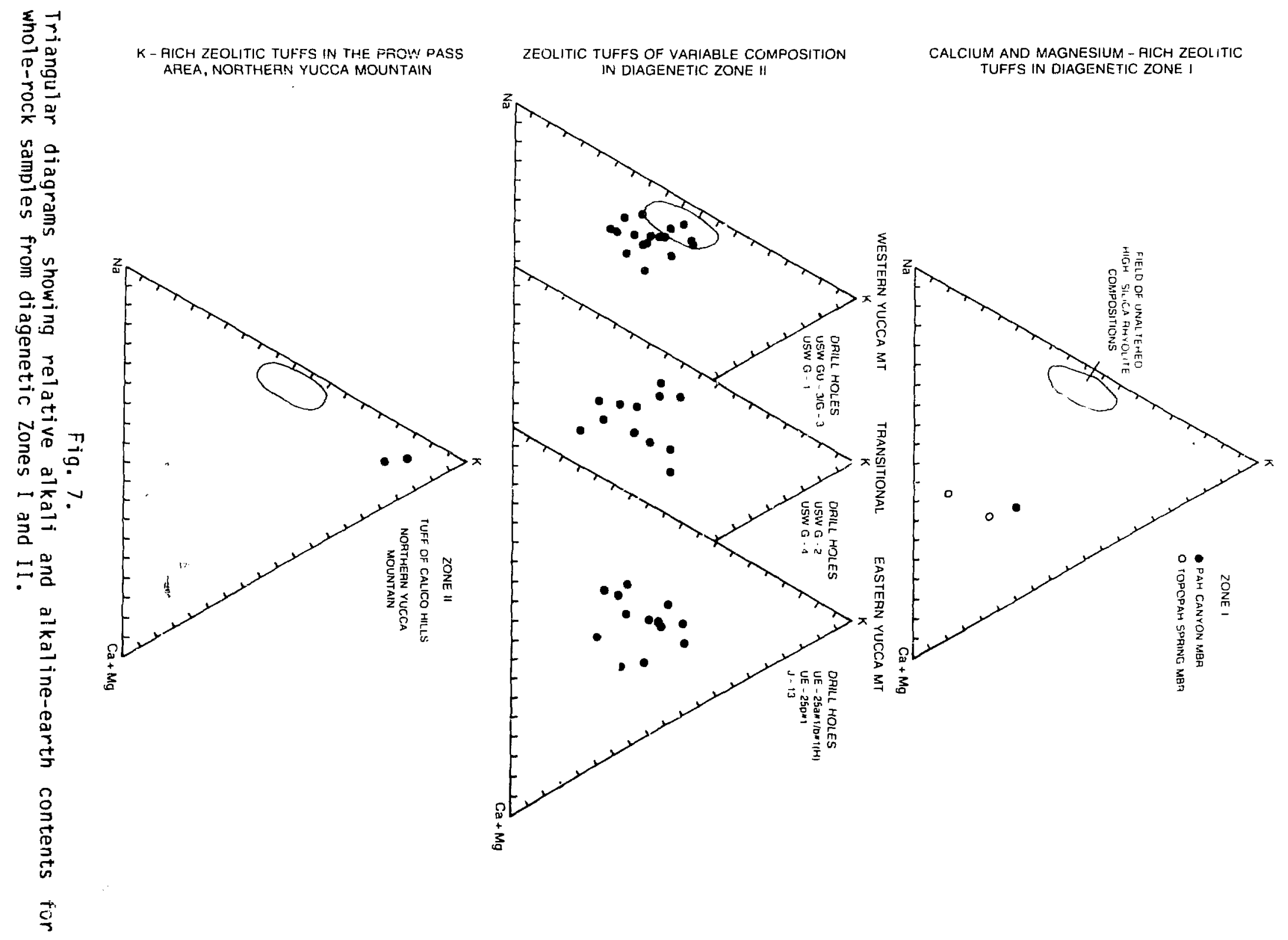


COMPARISON OF SELECTED TRACE ELEMENTS BETWEEN UNALTERED AND ALTERED TUFFS IN DIAGENETIC ZONE I, YUCCA MOUNTAIN, NEVADA

\begin{tabular}{|c|c|c|c|c|}
\hline & \multicolumn{2}{|c|}{$\begin{array}{l}\text { Pah Canyon Member, } \\
\text { Painibrush Tuff }\end{array}$} & \multicolumn{2}{|c|}{$\begin{array}{l}\text { Topopah Spring Member } \\
\text { Paintbrush Tuff }\end{array}$} \\
\hline & $\begin{array}{l}\text { Partially } \\
\text { welded } \\
\text { vitric tuff }\end{array}$ & $\begin{array}{l}\text { Partially } \\
\text { welded clay- } \\
\text { and zeolite- } \\
\text { rich tuff }\end{array}$ & $\begin{array}{l}\text { Unal tered } \\
\text { basal } \\
\text { vitrophyre }\end{array}$ & $\begin{array}{l}\text { Clay- and zeolite- } \\
\text { rich al tered basal } \\
\text { vitrophyre }\end{array}$ \\
\hline Sample No. & G2-547 & G2-675 & G1-1319 & G I- 1286 \\
\hline $\mathrm{Sr}$ & 290 & 1600 & $22^{b}$ & 1650 \\
\hline $\mathrm{Rb}$ & 141 & 87 & 1.54 & 52 \\
\hline U & 3.2 & 1.54 & 4.70 & 1.37 \\
\hline
\end{tabular}

dAाl concentrations in parts per million (ppm); data taken from Appendix $C$.

bSr value from Noble and Hedge (1969, Table I); the ANAA value for Sr in this sample is $760 \mathrm{ppm}$.

The altered tuffs in diagenetic Zones II, III, and IV are chemically more diverse than those of Zone I. Alkalies and alkaline earths in these tuffs vary systematically across Yucca Mountain. Tuffs on the eastern side of Yucca Mountain are characterized by calcium-rich compositions, whereas altered tuifs on the western side of Yucca Mountain are more alkali rich (Fig. 7). A transitional zone, characterized by samples from drill holes USW G-2 and G-4, separates the two compositional suites. The ialcium-rich tuffs on the eastern side of Yucca Mountain deviate most from original tuff compositions, being strongly enriched in calcium and magnesium and depleted in sodium and potassium. Alkali-rich tuffs from the western part of Yucca Mountain overlap original compositions to some extent, but many samples have higher sodium/potassium and calcium/sodium+potassium ratios than the unaltered tuffs. Although fewer compositions were determined for analcime-bearing tuffs in Zone II! and authigenic albite-bearing tuffs in Zone IV, these deeper zones of alteration show compositional variations similar to those found in Zone II. On the eastern side of Yucca Mountain, excess calclum in Zones III and IV is not incorborated into the primary authigenic minerals such as analcime and authigenic feldspar but instead results in a greater abundance of calcite. 
Potassium-rich tuffs crop out at Prow Pass, located at the northeri end of Yucca Mountain. Two samples from tuff of Calico Hills outcrops (sample \#62 and 63, Appendix A) contain 7.2 and $8.7 \mathrm{wt} \% \mathrm{~K}_{2} 0$ (on a volatile-free basis), respectively; by comparison, zeolitic tuffs beneath the exploration block, including those of the tuff of Calico Hills, generally contain less than 5.0 wt\% $\mathrm{K}_{2} \mathrm{O}$. The potassic tuffs occur in the structurally highest portion of diagenetic Zone II, which rises and thins northward at Yucca Mountain (Broxton et al. 1986). This unusual potassium enrichment apdears to be confined to the extreme northern end of Yucca Mountain because tuffs from the same stratigraphic horizon $3 \mathrm{~km}$ to the southeast, in drill hole USW G-2, contain only 3-4 wty $k_{2} 0$.

Glass and Mineral Compositions: Glass compositions were obtained for all members of the Paintbrush Tuff, the tuff of Calico Hills, and the Prow Pass Member of the Crater Flat Tuff (Appendix E). These glasses are high-silica rhyolites containing subequal potassium and sodium (Fig. 8). Si/Ai ratios for these glasses are relatively high (5.2 to 5.6) compared with those of clinoptilolites that replaced them $(2.6$ to 5.4$)$.

The compositions of glasses largely control the chemistry of pore waters during diagenesis. Selective leaching of sodium from glasses by groundwater during the initial stages of hydracion and diagenesis has been described by Lipman (1965), Noble (1967), and white et al. (1980). Though hydrated, the glasses in Zone I show no evidence for massive alkali exchange with groundwaters, suggesting that aiceration by groundwater has been limited and that most exchange is confined to the fine-grained dusty matrix where glass particles have much larger surface areas. The similarity of glass compositions throughout the stratigraphic sequence suggests that factors other than original glass compositions are resporsible for the chemical and mineralogic variations in diagenetically al tered tuffs at Yucca Mountain.

Clinodtilulite compositions (Appendix F) parallel the chemical trends described above for whole-rock compositions. Clinoptilolites in diagenetic Zone I are invariably calcium rich (Fig. 9) and have Si/Al ratios between 4.0 and 5.0 (Fig. 10). Magnesium contents of these zeolites are relatively high, ranging from 0.6 to $1.5 \%$ MgO by weight. Levy (1984) has shown that some of these zeolites have the thermal stability of heulandite (Mumpton 1960), but 
FIELD OF COMPOSITIONS FOR THE PAH CANYON MBR. $(n=3)$ TOPOPAH SPRING MBR. $(n=40)$ TUFF OF CALICO HIL'SS $(n=25)$ PROW PASS MBR. $(n=5)$

FIELD OF COMPOSITIONS FOR THE TIVA C., NYON MBR. $(n=17)$ YUCCA MOUNTAIN MBR. $(n=1)$

$\mathbf{n}=$ NUMBER OF ANALYSES

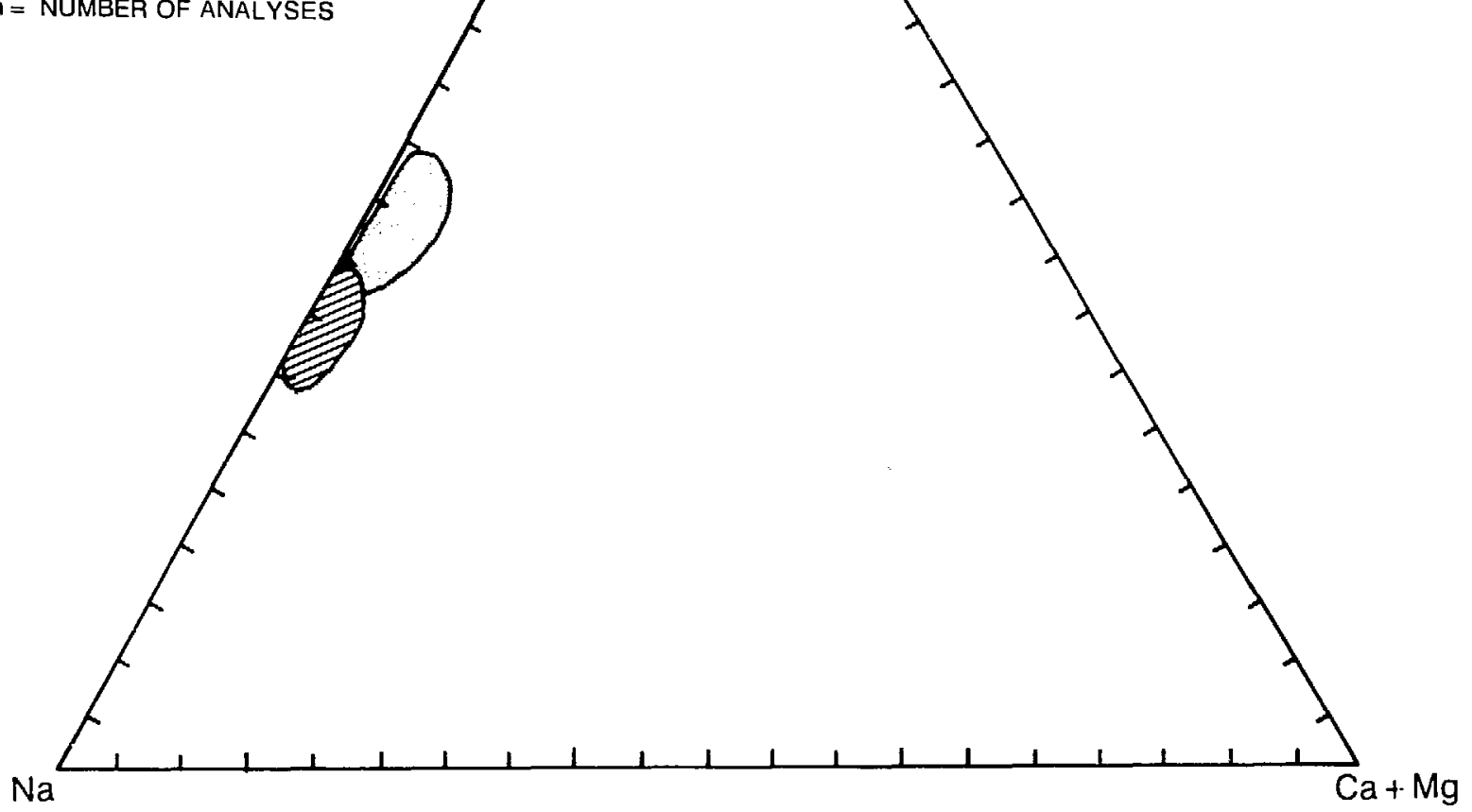

Fig. 8 .

Triangular diagram showing relative alkali and alkaline-earth contents for glasses from diagenetic Zone I, Yucca Mountain, Nevada.

others have a thermal stability intermediate between heulandite and clinoptilolite (Group 2 of Boles 1972). Calcic clinoptilolites at the top of the basal vitrophyre of the Topopah Spring Member are slightly more sodic than clinoptilolites within the shallow zeolite occurrence of the Pah Canyon Member in USW G-2 (Fig. 9).

Clinodilolite compositions in Zone II vary systematically both vertically and laterally at Yucca Mountain (Fig. 11). On the eastern side of Yucca Mountain, clinodtilolite compositions are calcic-potassic and show strong calcium enrichment with depth (Table III). Clinoptilolites on the western side of Yucca Mountain are sodic-dotassic and tend towards sodium 


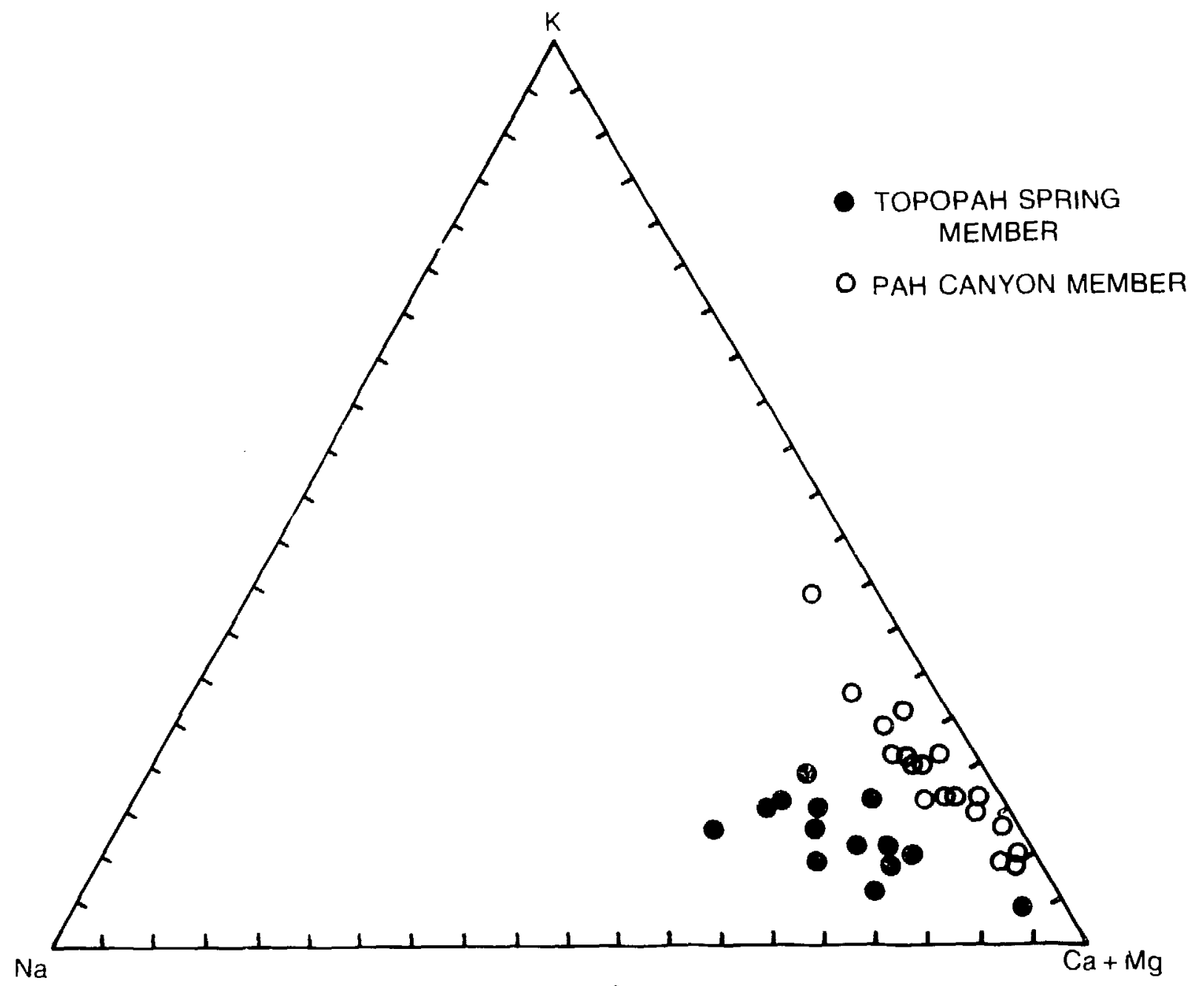

Fig. 9.

Trianqular diagram showing relative alkali and alkaline-earth contents for clinoptilolites of diagenetic Zone I, Yucca Mountain, Nevada.

enrichment with depth. A transitional zone, consisting of drill holes USW G-2, USW G-4, and USW H-4, has compositions that overlap both suites.

Clinoptilolites for the one sample reported for drill hole USW $\mathrm{H}-3$ are unusual in that they occur on the western side of Yucca Mountain, yet contain substantial calcium and little sodium. These clinodtilolites occur in the uppermost part of diagenetic Zone II and may reflect exchange of Zone II clinoptilolites with relatively calcium-rich solutions moving downward through Zone I (Levy 1984). The compositions of clinoptilolites from deeper portions of Zone II were also analyzed by Levy (1984), but those results were not included in Appendix $F$ or in $F i g .11$ of this report because the ratio of $(\mathrm{A})+\mathrm{Fe}) /(\mathrm{Na}+\mathrm{K}+2 \mathrm{Ca}+2 \mathrm{Mg})$ for these minerals was greater than 1.15 . However, the 


\section{CLINOPTILOLITES AND HEULANDITES}

IN DIAGENETIC ZONE I

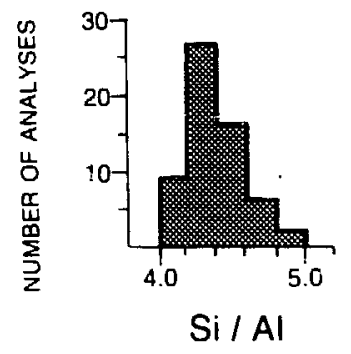

\section{EASTERN GROUP OF CALCIC CLINOPTILOLITES}
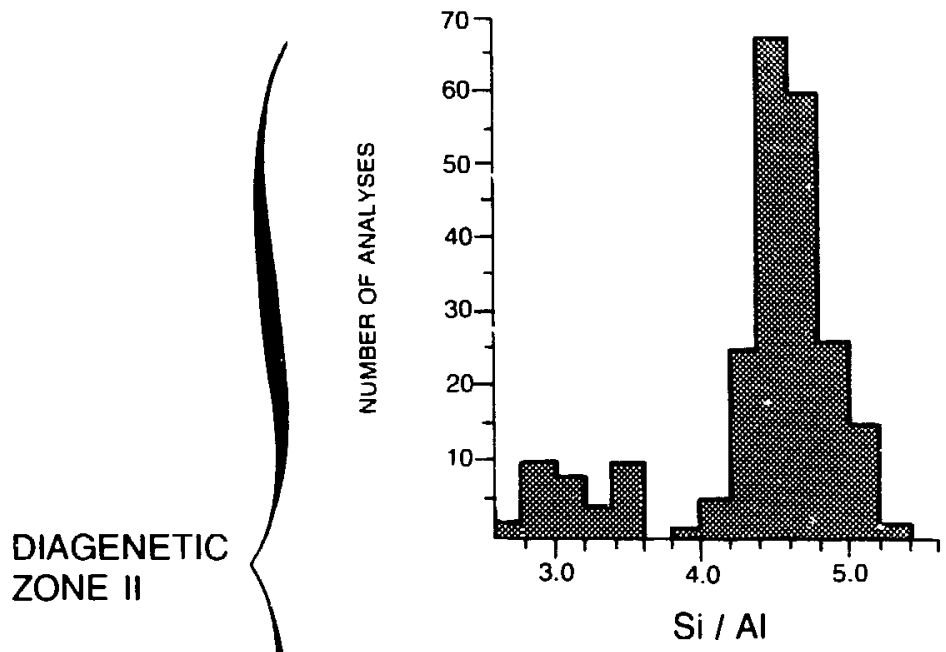

WESTERI . GROUP OF ALKALI CLINOPTILOLITES

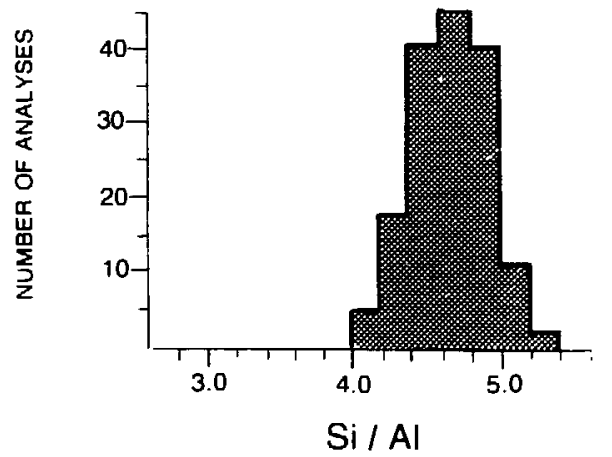

Fig. 10 .

Histogram comparing Si/Al ratios for clinoptilolites of Yucca Mountain, Nevada. 


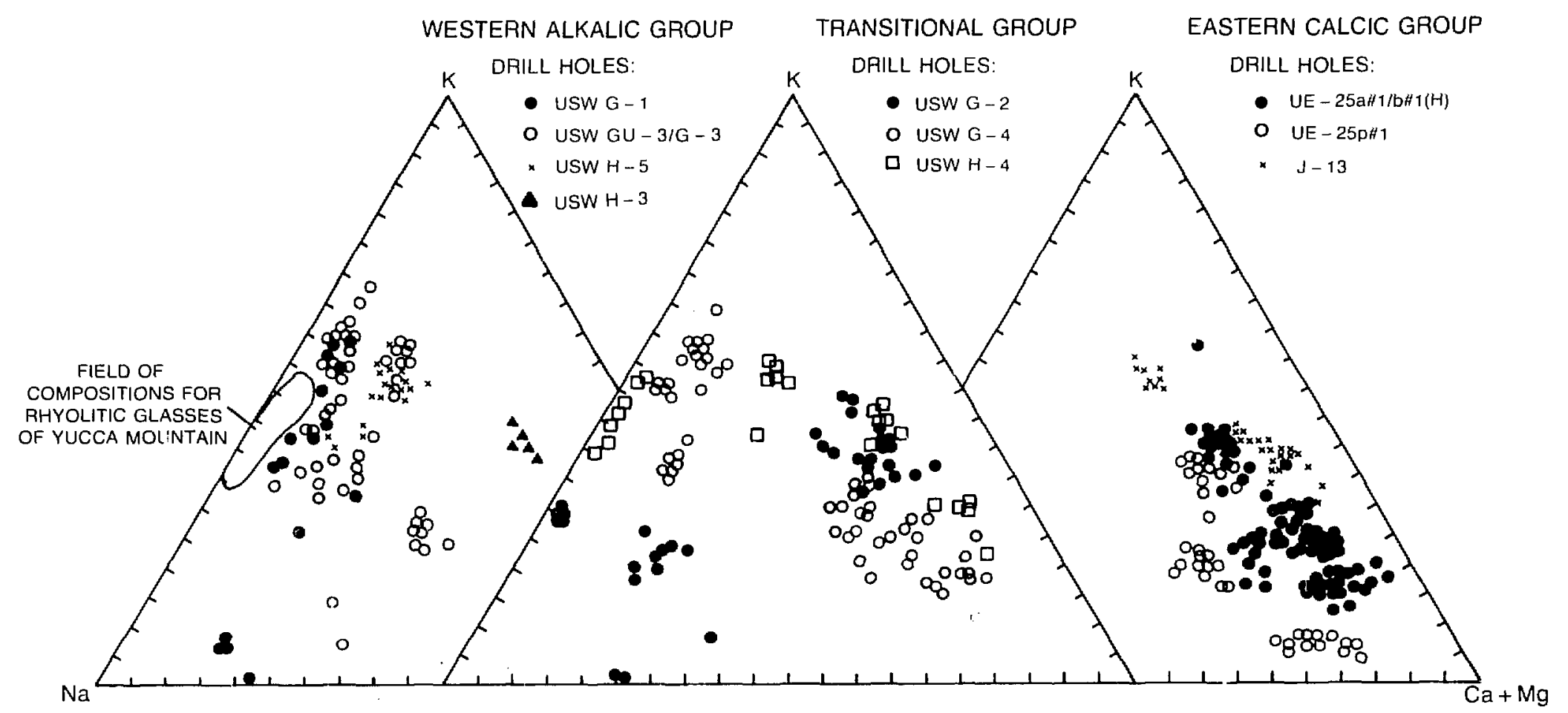

Fig. 11.

Triangular diagrams showing relative alkali and alkaline-earth contents for clinoptilolites of diagenetic Z Zones II and III, Yucca Mountain, Nevada. 
analyses by Levy clearly showed that the compositions of most clinoptilolites in Zone II of drill hole USW H-3 are sodic-potassic. In nearby orill hole USW G-3, a more complete suite of samples for diagenetic Zone II shows that analyses by Levy clearly showed that the compositions of most clinoptilolites in Zone II of drill hole USW H-3 are sodic-potassic. In nearby drill hole USW G-3, a more complete suite of samples for diagenetic Zone II shows that clinoptilolites in this part of Yucca Mountain are dominantly sodic-potassic in composition.

Si/Al ratios within clinoptilolites of diagenetic Zone II (Fig. 10) generally range between 3.8 and 5.4. However, Si/Al ratios within the eastern group of calcic clinoptilolites are bimodal with a small group of samples having $\mathrm{Si} / \mathrm{Al}$ ratios between 2.6 and 3.6 (Fig. 10). This small group of silica-poor compositions is associated with the most calcic clinoptilolites in the deepest parts of Zone. II.

Clinoptilolites associated with the potassic tuffs at the northern end of Yucca Mountain also have potassium-rich compositions. Clinoptilolite compositions were determined for three samples within the tuff of Calico Hills and for one sample in the overiying Topopah Soring Member, which is also zeolitic below the basal vitrophyre. These clinoptilolites tend to be potassic-calcic within the base of the Topopah Spring Member and the upper part of the tuff of Calico Hills. The clinoptilolites become increasingly potassic toward the center and base of the tuff cf Calico Hills (Fig. 12). Si/Al ratios are similar to those of other Zone II clinoptilolites, ranging between 4.0 and 5.0. Mordenite and adularia are common authigenic phases associated with these potassic clinoptilolites.

Analcimes in samples from Yucca Mountain range in molecular composition

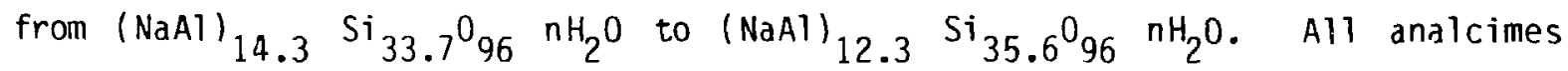
have nearly pure end-member compositions with only trace amounts of calcium and potassium substituting for sodium (Appendix G). Si/Al ratios for these analcimes range from 2.3 to 2.9 (Fig. 13). The distribution of Si/Al ratios appears to be bimodal, with the primary mode between 2.4 and 2.6 and a secondary mode between 2.7 and 2.8. The significance of bimodal Si/Al ratios in these analcimes is not known. However, these ranges of $\mathrm{Si} / \mathrm{Al}$ ratios are tyoical of analcimes associated with siliceous volcanic rocks (Coombs and Whetten 1967; Sheppard and Gude 1973). 


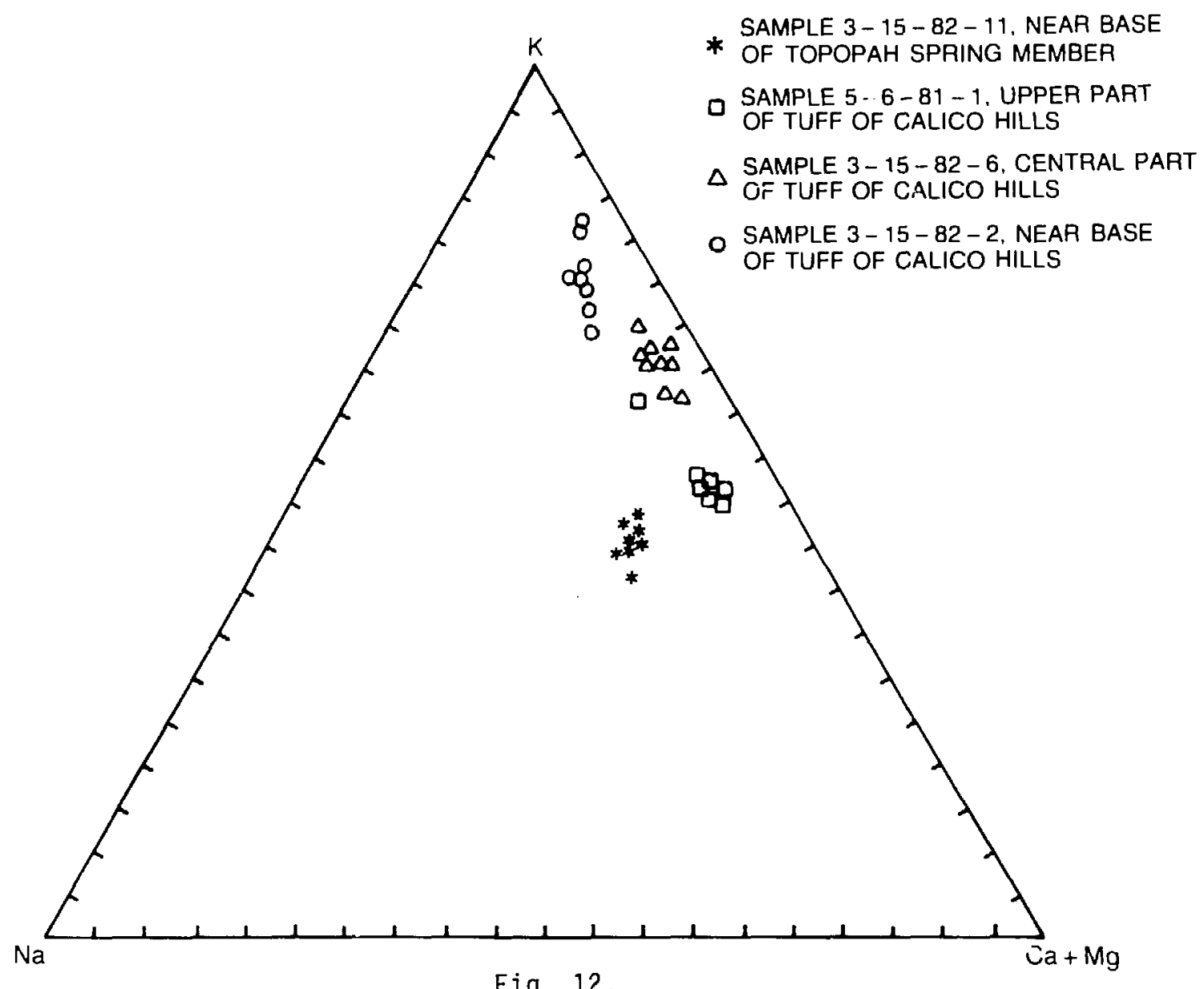

Fig. 12.

Triangular diagram showing relative alkali and alkaline-earth contents for clinoptilolites in the tuff of Calico Hills at Prow Pass, northern Yucca Mountain.

Authigenic potassium feldspar and albite compositions (Appendix $H$ ) are also characterized by nearly pure end-member compositions. These end-member compositions are typical for feldspars formed under diagenetic conditions (Kastner and Siever 1979). Most potassium feldspar compositions yield molecular formulae with 0.88 to 0.96 nontetrahedral cations per 8-oxygens (Fig. 14) rather than the ideal one nontetrahedral cation per 8-oxygens. Charge balance within these potassium feldspars is maintained because the tetrahedral framework is correspondingly aluminum poor and silicon rich. Si/Al ratios are 3.35 to 3.45 compared with an ideal ratio of $\leq 3.00$. Submicroscopic intergrowths of quartz and feldspars might account for the silica-rich chemistry of the feldspars. However, examination of several potassium feldspar grains by electron microscope revealed no evidence of intergrown quartz. 


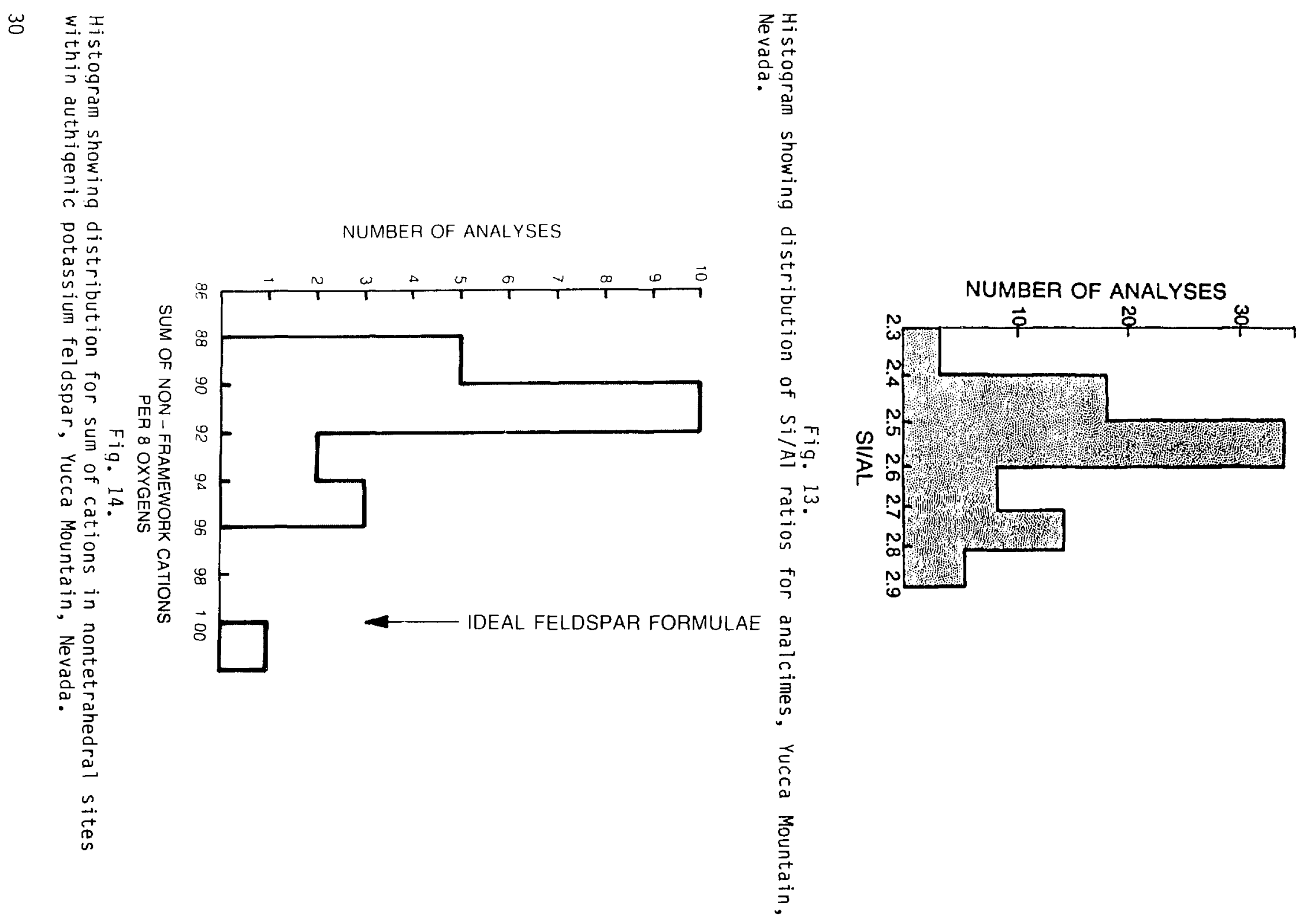




\section{DISCUSSION}

Vitric tuffs that were precursors to the major zeolite deposits of Yucca Mountain were generally high-silica rhyolites with similar major-element chemistries (Table II). Despite similar starting compositions, the principal zeolite deposits have extremely variable major-element chemistries. Individual stratigraphic units such as the tuff of Calico Hills display a wide range of compositions laterally across Yucca Mountain. Evidently diagenesis was accompanied by widespread mobilization and redistribution of chemical constituents within these tuffs. This widespread redistribution of elements within the tuffs supports Hoover's (1968) concept of diagenesis of tuffs at the Nevada Test Site within a chemically open system.

Groundwater is the likely medium through which chemical transport occurred. Cations in zeolites within the tuffs, particularly alkalies and alkaline earths, are readily exchanged with those in groundwaters. Therefore, systematic variations in the compositions of zeolitic tuffs probably reflect variable groundwater compositions during diagenesis. It is unclear, however, whether present-day tuff compositions represent original compositions acquired at the time of diagenetic crystallization or compositions acquired at a later date through ion exchange with younger groundwaters. Broxton et al. (1986) discuss possible origins of the chemical variability of tuffs at Yucca Mountain.

The zeolite occurrence at the top of the basal vitrophyre of the Topopah Spring member is the first major zeolite barrier that radioactive wastebearing solutions will encounter as they move downward through the unsaturated zone. The proximity of the zeolites within this interval to the potential repository makes them susceptible to contraction and dehydration during the thermal oul je expected after repository closure. This study and Levy's (1984) work indicate that this zeolite interval consistently bears calcium-rich clinoptilolite and heulandite. Calcium-rich clinoptilolites can lose up to $27 \%$ of their original water and contract in volume by $3 \%$ when heated to $100^{\circ} \mathrm{C}$ (Bish 1984, 1985).

The sorptive behavior of Zone I zeolites also may be compositionally dependent. Clay- and zeolite-rich tuffs in zone I are enriched in calcium, magnesium, and strontium and depleted in sodium, potassium, rubidium, and uranium. This may simply reflect the relative abundances of these elements in groundwaters of the unsaturated zone. However, it is al so oossible that zone 
I clinodilolites are sianificantly more selective for bivalent cations than for cations in other valence states. White et al. (1980) noted that groundwaters increase in sodium and decrease in calcium and magnesium after passing through zeolitic tuffs in the unsaturated zone at Rainier Mesa, located $50 \mathrm{~km}$ NNE of Yucca Mountain. They concluded that zeolites and clays selectively removed bivalent cations from the groundwater system. Similarly, zeolites on top of the basal vitrophyre in the Tododah Soring Member may have a greater selectivity for bivalent cations.

Clinoptilolite-bearing tuffs in zone II are the most important sorptive barriers to radionuclide migration at Yucca Mountain because of their great thicknesses, their abundant zeolites, and their position along potential groundwater pathways to the accessible environment. The thermal properties of Zone II zeolites are probably relatively unimportant for assessing repository Derformance because these zeolites lie a great distance below the repository level. Nonwelded tuffs at the base of the Topopah Spring Member and at the top of the tuff of Calico Hills are 75 to $100 \mathrm{~m}$ below the repository centerline and should not be significantly heated during the thermal pulse. Furthermore, these uppermost zeolites of Zone II contain substantial potassium, which minimizes thermal contraction and dehydration (Bish 1984, 1985).

Sorptive properties of clinootilolites in Zone II, particularly in the vicinity of the exploration block, are important for assessing repository Derformance because these zeolites occur along potential groundwater pathways from the repository to the accessible environment. These sorptive properties could vary with composition laterally and vertically across Yucca Mountain. Both sodium and calcium in clinoptilolite exchange readily with a wide range of Dotential radionuclides, including cesium, rubidium, strontium, and barium (Ames 1960; Breck 1974). Potassium, on the other hand, is more difficult to remove from the clinoptilolite structure, exchanging well with cesium and rubidium but exchanging poorly with strontium and barium (Ames 1960).

In the unsaturated zone, exchangeable cations within clinoptilolites on the western side of the exploration block consist primarily of sodium and potassium (Fig. 15). Clinodtilolites in the unsaturated zone on the eastern side of the exploratory block nave mixed sodium-potassium and calciumDotassium comoositions. In the saturated zone, clinoptilolite compositions tend to become more sodic with depth on the western side of Yucca Mountain and more calcic with depth to the east. Unfortunately, it is not possible to 


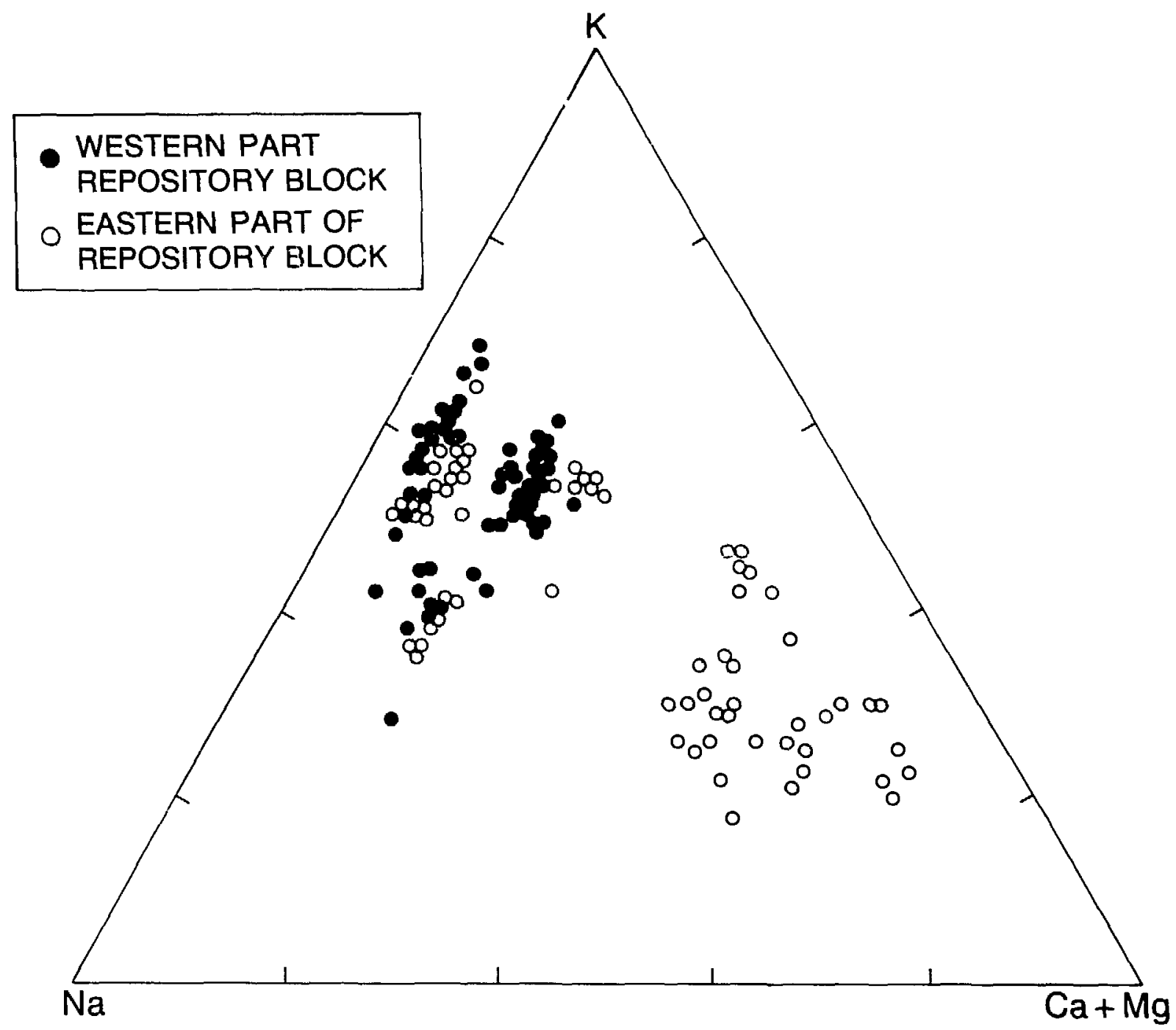

Fig. 15.

Triangular diagram showing alkali and alkaline-earth contents for clinoptilolites in the unsaturated zone below the exploration block, Yucca Mountain, Nevada.

accurately evaluate the sorption behavior of clinoptilolite as a function of its chemistry from published sorption experiments (Daniels et al. 1982; U.S. Department of Energy 1984). These experiments were designed to determine bulk sorption characteristics of Yucca Mountain tuffs and used whole-rock samples containing variable proportions of sorptive and nonsorptive minerals. In addition, the sorptive minerals had variable compositions. Further experiments are planned to determine the effect of exchangeable cation compositions on clinoptilolite sorption using monomineralic samples and end-member compositions. 
Potassic clinoptilolites are abundant only in outcrop at the north end of Yucca Mountain. These potassic clinoptilolites may have relatively puor sorption characteristics because of the difficulty in removing potassium from the zeolite structure. However, these potassic clinoptilolites do not occur along potential groundwater pathways from the repository to the accessible environment and their sorptive properties are not important for assessment of repository performance.

\section{SUMMARY AND CONCLUSIONS}

Major zeolite deposits at Yucca Mountain developed within thick sequences of nonwelded tuffs that were initially vitric. Alteration of these vitric tuffs resulted in the dissolution of the glass and precipitation of heulandite, clinoptilolite, and mordenite. These zeolite deposits are laterally extensive beneath much of Yucca Mountain and could provide important barriers to radionuclide migration.

The precursor vitric tuffs were primarily high-silica rhyolites with similar major element chemistry. However, zeolite deposits developed in these tuffs have variable compositions. Significant mobilization and redistribution of sodium, potassium, calcium, magnesium, and silicon during zeolitization indicates that diagenesis took place in an open chemical system.

Yucca Mountain tuffs can be divided into three compositional groups. In diagenetic Zone I, tuffs in thin zones of alteration have calcium- and magnesium-rich compositions. (2) In Zones II, III, and IV, diagenetically altered tuffs have compositions that vary laterally. These tuffs are calcic-potassic on the east side of Yucca Mountain but become increasingly sodic-potassic in stratigraphically equivalent units to the west. (3) Potassium-rich tuffs occur where Zone II crops out at Prow Pass in the northern part of Yucca Mountain. Clinoptilolites within each of these three compositional groups follow chemical trends similar to those found in their host tuffs.

Experimental studies have shown that variations in zeolite compositions can affect their thermal and exchange properties. At Yucca Mountain, clinoptilolites have exchangeable-cation compositions that span the entire compositional range between sodium, potassium, and calcium end members. Because of these compositional variations, the thermal expansion/contraction behavior, hydration/dehydration behavior, and ion-exchange properties of these 
zeolites and of their host tuffs may vary at Yucca Mouniain. Data presented in this study can be used in conjunction with experimental results to model the effects of mineral chemical variations on the physical properties of zeolites and their host tuffs in a redository environment.

\section{ACKNONLEDGMENTS}

We thank David Mann for preparation of polished thin sections used for microprobe studies. Barbara Hahn typed the manuscript and Anthony Garcia, Sharon Mikkelson, and Florence Fujita prepared the illustrations. We also thaik Donathon J. Krier and David T. Vaniman for their critical reviews of the manuscript.

\section{REFERENCES}

Ames, L. L., Jr., "The Cation jieve Properties of Clinoptilolite," Am. Minera1. 45, 689-700 (1960).

Bence, A. E., and A. L. Albee, "Empirical Correction Factors for Electron Microanalysis of Silicates and Oxides," J. Geol. 76, 382-403 (1968).

Bish, D. L., "Effects of Exchangeable Cation Composition on the Thermal Expansion/Contraction of Clinoptilolite," Clays and Clay Miner., 32, no. $6,444-462$ (1984).

Bish, D. L., "Effects of Composition on the Dehydration Behavior of Clinoptilolite and Heulandite," in "Proceedings for the International Conference on the Occurrence, Properties, and Utilization of Natural Zeol ites," Budapest, Hungary (1985).

Bish, D. L., and D. T. Vaniman, "Mineralogic Summary of Yucca Mountain, Nevada," Los Alamos National Laboratory report LA-10543-MS, 55 pp. (October 1985).

Boles, J. R., "Composition, Optical Properties, Cell Dimensions, and Thermal Stability of Some Heulandite Group Zeolites," Am. Mineral. 57, 1463-1493 (1972).

Breck, D. W., Zeolite Molecular Sieves: Structure, Chemistry, and Use (John Wiley and Sons, New York, 19741 .

Broxton, D. E., D. T. Vaniman, F. A. Caporuscio, B. H. Arney, and G. H. Heiken, "Detailed Petrographic and Microprobe Data for Drill Holes USW G-2 and UE25b-1H, Yucca Mountain, Nevada," Los Alamos National Laboratory report LA-9324-MS, 168 pp. (October 1982). 
Broxton, D. E., D. L. Bish, and R. G. Warren, "Distribution and Chemistry of Diagenetic Minerals at Yucca Mountain, Nye County, Nevada," Los Alamos National Laboratory document LA-UR-85-4032 (submitted to Clays and Clay Miner.).

Byers, F. M., Jr., W. J. Carr, P. P. Orkild, W. D. Quinlivan, and K. A. Sargent, "Volcanic Suites and Related Cauldrons of Timber Mountain-0asis Valley Caldera Complex, Southern Nevada," US Geological Survey Professional Paper 919, 70 pp. (1976).

Caporuscio, F., D. Vaniman, D. Bish, D. Broxton, B. Arney, G. Heiken, F. Byers, R. Gooley, and E. Semarge, "Petrologic Studies of Drill Cores USW G-2 and UE25b-1H, Yucca Mountain, Nevada," Los Alamos National Laboratory report LA-9255-MS, 111 pp. (April 1982).

Caporuscio, F. A., R. G. Warren, and D. E. Broxton, "Detailed Petrographic Descriptions and Microprobe Data for Tertiary Silicic Volcanic Rocks in Drill Hole USW G-1, Yucca Mountain, Nevada," Los Alamos National Laboratory report LA-9323-MS, 76 pp. (July 1985).

Christiansen, R. L., P. W. Lipman, P. P. Orkild, and F. M. Byers, Jr., "Structure of the Timber Mountain Caldera, Southern Nevada, and Its Relation to Basin-Range Structure," in "Geulog: cal Survey Research 1965," US Geological Survey Professional Paper 525B, B43-B48 (1965).

Christiansen, R. L., P. W. Lipman, W. J. Carr, F. M. Byers, Jr., P. P. Orkild, and K. A. Sargent, "Timber Mountain-Oasis Valley Caldera Complex of Southern Nevada," Geol. Soc. Am. Bu11. 88, 943-959 (1977).

Coombs, D. S., and J. T. Whetten, "Composition of Analcime from Sedimentary and Burial Metamorphic Rocks," Geol. Soc. Am. Bu11. 78, 269-282 (1967).

Daniels, W. R., K. Wolfsberg, R. S. Rundberg, A. E. Ogard, J. F. Kerrisk, et a1., "Summary Report on the Geochemistry of Yucca Mountain and Environs," Los Alamos National Laboratory report LA-9328-MS, 364 pp. (December 1982).

Garcia, S. R., W. K. Hensley, M. M. Minor, M. M. Denton, and M. A. Fuka, "An Automated Multidetector System for Instrumental Neutron Activation Analysis of Geological and Environmental Materials," in Atomic and Nuclear Methods in Fossil Energy Research, R. H. Filby, B. S. Carpenter, and R. C. Ragaini, Eds. (P1enum Press, New York, 1982) 133-140.

Hay, R. L., "Stratigraphy and Zeolite Diagenesis of the John Day Formation of Oregon," Univ. Calif. Publ. Geol. Sci. 42, 199-262 (1963).

Heiken, G. H., and M. L. Bevier, "Petrology of Tuff Units from the J-13 Drill Site, Jackass Flats, Nevada," Los Alamos Scientific Laboratory report LA-7563-MS, 55 pp. (February 1979).

Hoover, D. L., "Genesis of Zeolites, Nevada Test Site," E. B. Eckel, Ed., Geol. Soc. Am. Mem. 110, 275-284 (1968).

Iijima, A., "Effect of Pore Water to Clinoptilolite - Analcime - Albite Reaction Series," J. Fac. Sci., Univ. Tokyo, Sec. II, 19, 133-147 (1975). 
Iijima, A., "Geologic Occurrences of Zeolites in Marine Environments," in Natural Zeolites, Occurrence, Properties, Use, L. B. Sand and F. A. Mumpton, Eds. (Pergamon Press, Elms ford, New York, 1978) 175-198.

Iijima A., "Geology of Natural Zeolites and Zeolitic Rocks," in Proceedings of the Fifth International Conference on Zeolites, L. V. Rees, Ed. (Heyden, London, 1980 103-118.

Johnstone, J. K. and K. Wolfsberg, Eds., "Evaluation of Tuff as a Medium for a Nuclear Waste Repository: Interim Status Report on the Properties of Tuff," Sandia National Laboratories report SAND 80-1464 (1980).

Kastner, M., and R. Siever, "Low Temperature Feldspars in Sedimentary Rocks," Am. J. Sci. 279, 435-479 (1979).

Levy, S. S., "Petrology of Samples from Drill Holes USW H-3, H-4, and H-5, Yucca Mountain, Nevada," Los Alamos National Laboratory report LA-9706-MS, 77 pp. (June 1984).

Lipman, P. W., R. L. Christiansen, and J. T. O'Conner, "A Compositionally Zoned Ash-Flow Sheet in Southern Nevada," US Geological Survey Professional Pàper 524-F, F1-F47 (1966).

Lipman, P. W., "Chemical Comparison of Glassy and Crystalline Volcanic Rocks," US Geological Survey Bulletin 1201-D, 24 pp. (1965).

Maldonado, F., and S. L. Koether, "Stratigraphy, Structure, and Some Petrographic Features of Tertiary Volcanic Rocks at the USW G-2 Drill Hole, Yucca Mountain, Nye County, Nevada," US Geological Survey open-file report 83-732, $83 \mathrm{pp}$. (1983).

Minor, M. M., W. K. Hensley, M. M. Denton, and S. R. Garcia, "An Automated Activation Analysis System," J. Radioanal. Chem. 70, 459-471 (1982).

Mumpton, F. A., "Clinodtilolite Redefined," Am. Minerã . 45, 351-369 (1960).

Noble, D. C., "Sodium, Potassium, and Ferrous Iron Contents of Some Secondarily Hydrated Natural Silicic Glasses," Am. Mineral. 52, 280-286 (1967).

Noble, D. C., and C. E. Hedge, " ${ }^{87} \mathrm{Sr} /{ }^{86} \mathrm{Sr}$ Variations Within Individual Ash-Flow Sheets," US Geological Survey Professionai Paper 650-C, C133-C139 (1969).

Quinlivan, W. D., and F. M. Byers, Jr., "Chemical Data and Variation Diagrams of Igneous Rocks from the Timber Mountain-0asis Valley Caldera Complex, Southern Nevada," US Geological Survey open-file report $77-724,9$ pD. (1977).

Scott, R., and M. Castellanos, "Preliminary Report on the Geologic Character of the Drill Holes USW GU-3 and USW G-3," US Geological Survey open-file report 84-491, 121 DD. (1984). 
Sheppard, R. A., and A. J. Gude, 3rd., "Zeolites and Associated Authigenic Silicate Minerals in Tuffaceous Rocks of the Big Sandy Formation, Mohave County, Arizona," US Geological Survey Professional Paper 830, 36 pp. (1973).

Spengler, R. W., D. C. Muller, and R. B. Livermore, "Preliminary Report on the Geology and Geophysics of Drill Hole UE25a-1, Yucca Mountain, Nevada," US Geological Survey open-file report 79-1244, 43 pp. (1979).

Spengler, R. W., F. M. Byers, Jr., and J. B. Warner, "Stratigraphy and Structure of Volcanic Rocks in Drill Hole USW G1, Yucca Mountain, Nye County, Nevada," US Geological Survey open-file report 81-1349, 50 pp. (1981).

Sykes, M. L., G. H. Heiken, and J. R. Smyth, "Mineralogy and Petrology of Tuff Units from the UE25a-1 Drill Site, Yucca Mountain, Nevada," Los Alamos Scientific Laboratory report LA-8139-MS, 76 pp. (November 1979).

US Department of Energy, "Draft Environmental Assessment, Yucca Mountain Site, Nevada Research and Development Area, Nevada," Ctice of Civilian Radioactive Waste Management report, DOE/RW-0012 (1984).

Valentine, G., "Procedures for Analysis of Silicate Rocks and Minerals at Los Alamos National Laboratory by X-Ray Fluorescence," Los Alamos National Laboratory report LA-9663-MS, 33 pp. (May 1983).

Vaniman, D., D. Bish, D. Broxton, F. Byers, G. Heiken, B. Carlos, E. Semarge, $F$. Caporuscio, and R. Gooley, "Variations in Authigenic Mineralogy and Sorptive Zeolite Abundance at Yucca Mountain, Nevada, Based on Studies of Drill Cores USW GU-3 and G3," Los Alamos National Láboratory report LA-9707-MS, $71 \mathrm{pp}$. (June 1984).

White, A. F., H. C. Claassen, and L. V. Benson, "The Effect of Dissolution of Volcanic Glass on the Water Chemistry in a Tuffaceous Aquifer, Rainier Mesa, Nevada," US Geological Survey Water-Supply Paper 1535-Q, Q1-034 (1980) .

Zielinski, R. A., "Evaluation of Ash-flow Tuffs as Hosts for Radioactive Waste: Criteria Based on Selective Leaching of Manganese 0xides," US Geological Survey open-file report $83-480,21 \mathrm{pp} .(1983)$. 


\section{MAJOR-ELEMENT ANALYSES OF TUFFS BY X-RAY FLUORESCENCE} YUCCA MOUNTAIN, NEVADA ${ }^{a}$

\begin{tabular}{|c|c|c|c|c|c|c|c|}
\hline \multirow{2}{*}{$\begin{array}{l}\text { Sample } \\
\text { Number }\end{array}$} & \multicolumn{6}{|c|}{ Drill Hole USW G-2 } & \multirow[b]{2}{*}{7.} \\
\hline & 1. & 2. & 3. & 4. & 5. & $G$. & \\
\hline Depth (ft) & 675 & 2430 & 2667 & 3067 & 3192 & 3454 & 3541 \\
\hline $\begin{array}{l}\mathrm{SiO} 2 \\
\mathrm{TiO} 2 \\
\mathrm{Al} 203 \\
\mathrm{Fe} 203 \\
\mathrm{MnO} \\
\mathrm{MgO} \\
\mathrm{CaO} \\
\mathrm{Na} 20 \\
\mathrm{~K} 20 \\
\mathrm{P} 205 \\
\mathrm{LOI}\end{array}$ & $\begin{array}{c}64.3 \\
0.33 \\
16.8 \\
1.83 \\
0.17 \\
1.77 \\
2.60 \\
1.38 \\
2.72 \\
0.05 \\
8.0\end{array}$ & $\begin{array}{c}76.5 \\
0.14 \\
11.9 \\
1.24 \\
0.13 \\
0.41 \\
1.98 \\
1.68 \\
3.95 \\
0.01 \\
3.49\end{array}$ & $\begin{array}{c}75.6 \\
0.11 \\
12.7 \\
1.38 \\
0.08 \\
0.14 \\
1.59 \\
2.51 \\
3.04 \\
0.02 \\
2.44\end{array}$ & $\begin{array}{c}73.3 \\
0.16 \\
13.1 \\
1.44 \\
0.04 \\
0.29 \\
2.25 \\
3.37 \\
3.34 \\
0.05 \\
3.61\end{array}$ & $\begin{array}{c}76.0 \\
0.12 \\
11.9 \\
1.34 \\
0.08 \\
0.14 \\
1.11 \\
2.63 \\
3.89 \\
0.02 \\
2.89\end{array}$ & $\begin{array}{c}75.0 \\
0.14 \\
14.3 \\
1.60 \\
0.04 \\
0.57 \\
1.49 \\
2.96 \\
2.12 \\
0.02 \\
2.73\end{array}$ & $\begin{array}{c}73 . E \\
0.2 \\
14.0 \\
1.96 \\
0.08 \\
0.4 j \\
0.74 \\
4.17 \\
3.37 \\
0.03 \\
2.14\end{array}$ \\
\hline Total & 100.0 & 101.4 & 99.5 & 101.0 & 100.2 & 100.9 & 100.9 \\
\hline $\begin{array}{l}\mathrm{Si} \\
\mathrm{Ti} \\
\mathrm{Al} \\
\mathrm{Fe}+3 \\
\mathrm{Mn} \\
\mathrm{Mg} \\
\mathrm{Ca} \\
\mathrm{Na} \\
\mathrm{K} \\
\mathrm{F} \\
\mathrm{Ca}+\mathrm{Mg} \\
\mathrm{K} \\
\mathrm{Na}\end{array}$ & $\begin{array}{c}66.0 \\
0.25 \\
20.3 \\
1.41 \\
0.12 \\
2.71 \\
2.86 \\
2.75 \\
3.56 \\
0.04 \\
46.9 \\
30.0 \\
23.1\end{array}$ & $\begin{array}{c}74.5 \\
0.10 \\
13.7 \\
0.91 \\
0.09 \\
0.59 \\
2.07 \\
3.17 \\
4.91 \\
0.01 \\
24.8 \\
45.7 \\
29.5\end{array}$ & $\begin{array}{c} \\
73.8 \\
0.08 \\
14.6 \\
1.01 \\
0.05 \\
0.20 \\
1.66 \\
4.75 \\
3.79 \\
0.02 \\
17.9 \\
36.4 \\
45.7\end{array}$ & $\begin{array}{c}70.7 \\
0.12 \\
14.9 \\
1.04 \\
0.03 \\
0.42 \\
2.32 \\
6.30 \\
4.11 \\
0.04 \\
20.8 \\
31.2 \\
47.9\end{array}$ & $\begin{array}{c}74.0 \\
0.09 \\
13.7 \\
0.98 \\
0.05 \\
0.20 \\
1.16 \\
4.96 \\
4.83 \\
0.02 \\
12.2 \\
43.3 \\
44.5\end{array}$ & $\begin{array}{c}72.0 \\
0.10 \\
16.2 \\
1.16 \\
0.03 \\
0.82 \\
1.53 \\
5.51 \\
2.60 \\
0.02 \\
22.5 \\
24.8 \\
52.7\end{array}$ & $\begin{array}{c}69.7 \\
0.14 \\
15.6 \\
1.39 \\
0.05 \\
0.63 \\
0.75 \\
7.64 \\
4.06 \\
0.02 \\
10.6 \\
31.0 \\
58.4\end{array}$ \\
\hline
\end{tabular}

Sample Descriptions:

1. Pah Canyon Member, Paintbrush Tuff. Clinoptilolite- and smectite-bearing nonwelded tuff in diagenetic Zone $I$.

2. Tuff of Calico Hills. Clinoptilolite- and mordenite-bearing nonwelded tuff in diagenetic Zone II.

3. Tuff of Calico Hills. Clinoptilolite- and mordenite-bearing nonwelded tuff in diagenetic Zone II.

4. Prow Pass Member, Crater Flat Tuff. Mordenite-bearing tuff in diagenetic Zone II.

5. Prow Pass Member, Crater Flat Tuff. Clinoptilolite-and mordenite-bearing tufí in diagenetic Zone II.

6. Bullfrog Member, Crater Flat Tuff. Mordenite-bearing tuff in diagenetic Zone III.

7. Bullfrog Member, Crater Flat Tuff. Analcime-bearing tuff in diagenetic Zone III.

a All sodium concentrations determined by atomic absorption or plasma source

b emission spectrophotometry.

LOI not measured but determined by difference in analytical total from $100 \%$. 


\section{APPENDIX A (cont)}

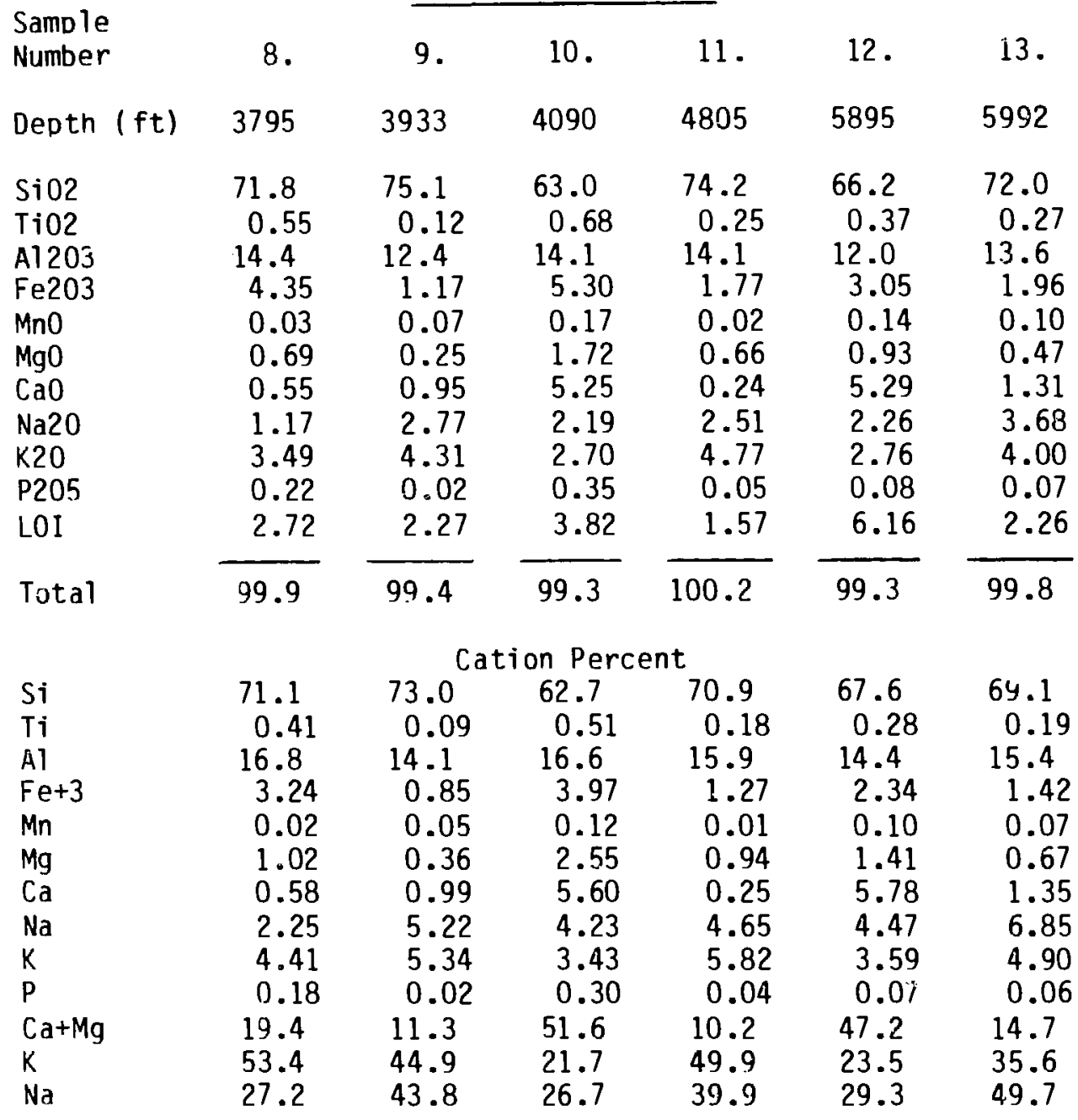

Orill Hole USW G-2

Sample Descriptions:

8. Tram Member, Crater Flat Tuff, Analcime- and kaolinitebearing nonwelded tuff in diagenetic Zone III.

9. Tram Member, Crater Flat Tuff. Analcime-bearing tuff in diagenetic Zone III.

10. Rhyodacite Lava. Analcime- and calcite-bearing lava in diagenetic Zone III.

11. Tuff of Lithic Ridge. Authigenic albite- and analcimebearing nonwelded tuff in diagenetic Zone IV.

12. Bedded Tuff. Calcite- and chlorite-bearing bedded tuff in diagenetic Zone IV.

i3. 01der Tuff Sequence. Calcite- and chlorite-bearing nonwelded tuff in diagenetic Zone IV. 


\section{Drill Holes USW GU-3 and USW G-3}

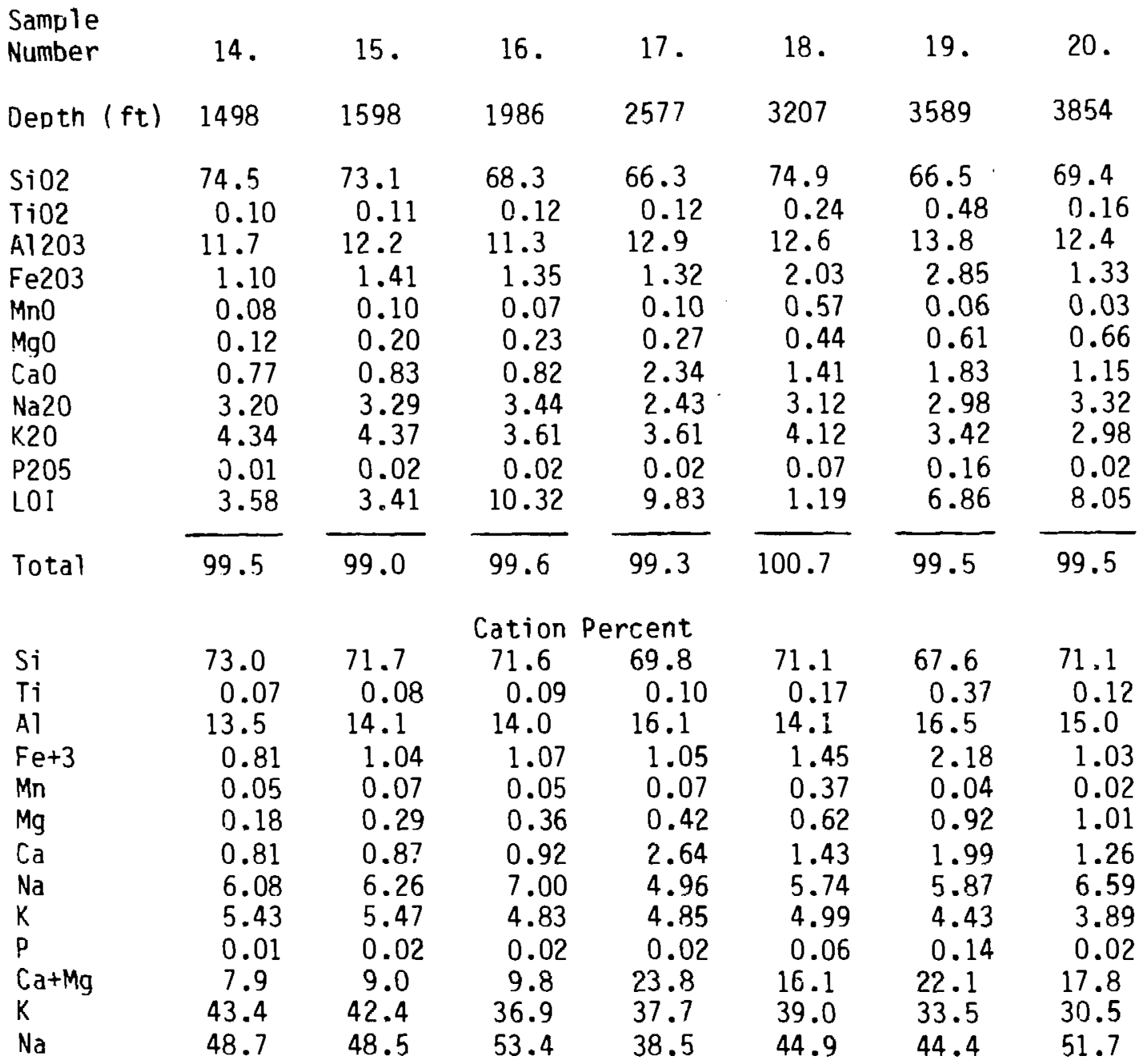

Sample Descriptions:

14. Tuff of Calico Hills. Vitric nonwelded tuff in diagenetic Zone I. 15. Prow Pass Member, Crater Flat Tuff. Vitric nonwelded tuff in diagenetic Zone I.

16. Prow Pass Member, Crater Flat Tuff. Clinoptilolite-bearing nonwelded tuff in diagenetic Zone II.

17. Bullfrog Member, Crater Flat Tuff. Clinoptilolite-bearing nonwelded tuff in diagenetic Zone II.

18. Tram Member, Crater Flat Tuff. Clinoptilolite-bearing partially welded tuff in diagenetic Zone II.

19. Tram Member, Crater Flat Tuff. Clinodtilolite-bearing nonwelded tuff in diagenetic Zone II.

20. Tuff of Lithic Ridge. Clinoptilolite-bearing nonwelded tuff in diagenetic Zone II. 
Drill Hole USW G-3

Samole

Number

21. 22. 23.24 .25 .

$\begin{array}{lccccc}\text { Dedth (ft) } & 4008 & 4263 & 4568 & 4786 & 4869 \\ & & & & & \\ \text { Si02 } & 69.8 & 73.2 & 67.8 & 72.2 & 71.0 \\ \text { Ti02 } & 0.34 & 0.26 & 0.43 & 0.26 & 0.23 \\ \text { A1203 } & 14.4 & 12.7 & 13.7 & 13.6 & 12.9 \\ \text { Fe203 } & 2.29 & 1.75 & 2.96 & 1.77 & 1.58 \\ \text { Mn0 } & 0.07 & 0.07 & 0.08 & 0.11 & 0.05 \\ \text { Mg0 } & 0.60 & 0.48 & 1.08 & 0.61 & 0.50 \\ \text { Ca0 } & 1.31 & 1.27 & 1.80 & 1.25 & 0.96 \\ \text { Na20 } & 3.44 & 4.00 & 3.56 & 3.39 & 4.70 \\ \text { K20 } & 4.85 & 3.55 & 2.40 & 4.23 & 2.57 \\ \text { P205 } & 0.09 & 0.05 & 0.11 & 0.05 & 0.04 \\ \text { L01 } & 1.83 & 2.61 & 5.25 & 2.41 & 4.11 \\ \text { Tota1 } & \text { 99.0 } & \underline{99.9} & \underline{99.2} & \underline{99.9} & 98.6\end{array}$

Cation Percent

$\begin{array}{lccccc}\mathrm{Si} & 67.1 & 70.3 & 67.7 & 69.3 & 69.8 \\ \mathrm{Ti} & 0.25 & 0.19 & 0.32 & 0.19 & 0.17 \\ \mathrm{Al} & 16.3 & 14.4 & 16.1 & 15.4 & 14.9 \\ \mathrm{Fe}+3 & 1.66 & 1.27 & 2.22 & 1.28 & 1.17 \\ \mathrm{Mn} & 0.05 & 0.05 & 0.06 & 0.07 & 0.03 \\ \mathrm{Mg} & 0.86 & 0.69 & 1.61 & 0.87 & 0.73 \\ \mathrm{Ca} & 1.35 & 1.31 & 1.93 & 1.29 & 1.01 \\ \mathrm{Na} & 6.41 & 7.45 & 6.89 & 6.31 & 8.95 \\ \mathrm{~K} & 5.95 & 4.35 & 3.06 & 5.18 & 3.22 \\ \mathrm{P} & 0.07 & 0.04 & 0.09 & 0.04 & 0.03 \\ \mathrm{Ca}+\mathrm{Mg} & 15.2 & 14.5 & 26.2 & 15.8 & 12.5 \\ \mathrm{~K} & 40.8 & 31.5 & 22.7 & 38.0 & 23.1 \\ \mathrm{Na} & 44.0 & 54.0 & 51.1 & 46.2 & 64.3\end{array}$

Sample Descriptions:

21. Tuff of Lithic Ridge. Analcime-bearing nonwelded tuff in diagenetic Zone III.

22. Tuff of Lithic Ridge. Analcime-bearing nonwelded tuff in diagenetic Zone III.

23. Tuff of Lithic Ridge. Analcime-bearing nonwelded tuff in diagenetic Zone III.

24. 01der Tuff Sequence. Analcime-bearing nonwelded tuff in diagenetic Zone III.

25. 01der Tuff Sequence. Analcime-bearing nonwelded tuff in diagenetic Zone III. 
Drill Hole USW G-4

\begin{tabular}{|c|c|c|c|c|c|c|c|}
\hline $\begin{array}{l}\text { Sample } \\
\text { Number }\end{array}$ & 26. & 27 . & 28 . & 29. & 30. & 31. & 32. \\
\hline Depth $(f t)$ & 1314 & 1470 & 1544 & 2131 & 2226 & 2716 & 2792 \\
\hline $\begin{array}{l}\mathrm{SiO} 2 \\
\mathrm{TiO} 02 \\
\mathrm{~A} 1203 \\
\mathrm{Fe} 203 \\
\mathrm{MnO} \\
\mathrm{MgO} \\
\mathrm{CaO} \\
\mathrm{Na} 20 \\
\mathrm{~K} 20 \\
\mathrm{P} 205 \\
\mathrm{LOI}\end{array}$ & $\begin{array}{c}70.2 \\
0.14 \\
16.7 \\
1.44 \\
0.09 \\
0.90 \\
3.51 \\
1.90 \\
0.72 \\
0.02 \\
5.91\end{array}$ & $\begin{array}{c}69.5 \\
0.07 \\
10.9 \\
0.90 \\
0.05 \\
0.05 \\
0.89 \\
2.53 \\
4.31 \\
0.02 \\
10.10\end{array}$ & $\begin{array}{c}69.3 \\
0.07 \\
11.3 \\
0.96 \\
0.04 \\
0.06 \\
0.84 \\
2.85 \\
4.00 \\
0.02 \\
9.90\end{array}$ & $\begin{array}{c}65.1 \\
0.11 \\
13.0 \\
1.33 \\
0.02 \\
0.17 \\
2.62 \\
2.46 \\
3.39 \\
0.01 \\
10.56\end{array}$ & $\begin{array}{c}66.4 \\
0.14 \\
13.1 \\
1.45 \\
0.05 \\
0.25 \\
2.43 \\
2.15 \\
3.78 \\
0.01 \\
9.40\end{array}$ & $\begin{array}{c}67.7 \\
0.13 \\
12.2 \\
1.24 \\
0.04 \\
0.20 \\
2.57 \\
2.81 \\
2.40 \\
0.01 \\
10.11\end{array}$ & $\begin{array}{c}68.3 \\
0.16 \\
11.6 \\
1.32 \\
0.03 \\
0.24 \\
2.94 \\
2.68 \\
1.63 \\
0.02 \\
10.98\end{array}$ \\
\hline Total & 101.5 & 99.3 & 99.4 & 98.8 & 99.2 & 99.5 & 99.9 \\
\hline $\begin{array}{l}\mathrm{Si} \\
\mathrm{Ti} \\
\mathrm{Al} \\
\mathrm{Fe}+3 \\
\mathrm{Mn} \\
\mathrm{Mg} \\
\mathrm{Ca} \\
\mathrm{Na} \\
\mathrm{K} \\
\mathrm{P} \\
\mathrm{Ca}+\mathrm{Mg} \\
\mathrm{K} \\
\mathrm{Na}\end{array}$ & $\begin{array}{c}69.6 \\
0.10 \\
19.5 \\
1.07 \\
0.06 \\
1.33 \\
3.73 \\
3.65 \\
0.91 \\
0.02 \\
52.6 \\
9.5 \\
38.0\end{array}$ & $\begin{array}{c}73.5 \\
0.06 \\
13.6 \\
0.72 \\
0.04 \\
0.08 \\
1.01 \\
5.19 \\
5.82 \\
0.02 \\
9.0 \\
48.1 \\
42.9\end{array}$ & $\begin{array}{c}\text { Cat } \\
72.9 \\
0.06 \\
14.1 \\
0.76 \\
0.03 \\
0.09 \\
0.95 \\
5.81 \\
5.36 \\
0.02 \\
8.5 \\
43.9 \\
47.6\end{array}$ & $\begin{array}{c}\text { Perce } \\
69.5 \\
0.09 \\
16.4 \\
1.07 \\
0.01 \\
0.27 \\
3.00 \\
5.09 \\
4.62 \\
0.01 \\
25.2 \\
35.6 \\
39.2\end{array}$ & $\begin{array}{c}69.8 \\
0.11 \\
16.3 \\
1.15 \\
0.04 \\
0.39 \\
2.74 \\
4.38 \\
5.07 \\
0.01 \\
24.9 \\
40.3 \\
34.8\end{array}$ & $\begin{array}{c}71.5 \\
0.10 \\
15.2 \\
0.98 \\
0.03 \\
0.31 \\
2.91 \\
5.75 \\
3.23 \\
0.01 \\
26.4 \\
26.5 \\
47.1\end{array}$ & $\begin{array}{c}72.7 \\
0.13 \\
14.6 \\
1.06 \\
0.02 \\
0.38 \\
3.35 \\
5.53 \\
2.21 \\
0.02 \\
32.5 \\
19.3 \\
48.2\end{array}$ \\
\hline
\end{tabular}

Sample Descriptions:

26. Topopah Spring Member, Paintbrush Tuff. Smectite- and clinoptilolitebearing tuff at top of Topopah Spring vitrophyre in diagenetic Zone I.

27. Tuff of Calico Hills. Clinoptilolite-bearing nonwelded tuff in diagenetic Zone 11 .

28. Tuff of Calico Hills. Clinoptilolite- and mordenite-bearing nonwelded tuff in diagenetic Zone II.

29. Prow Pass Member, Crater Flat Tuff. Clinoptilolite- and mordenite-bearing nonwelded tuff in diagenetic Zone II.

30. Prow Pass Member, Crater Flat Tuff. Clinoptilolite- and mordenite-bearing nonwelded tuff in diagenetic Zone II.

31. Bullfrog Member, Crater Flat Tuff. Mordenite-bearing nonwelded tuff in diagenetic Zone II.

32. Tram Member, Crater Flat Tuff. Mordenite-bearing nonwelded tuff in diagenetic Zone II. 
Drill Hole USW G-4

Sample

Number

33.

34 .

$35 . \quad 36$.

37.

38.

39.

\begin{tabular}{|c|c|c|c|c|c|c|c|}
\hline $\begin{array}{l}\text { Depth }(\mathrm{ft}) \\
\text { Si02 } \\
\text { Ti02 } \\
\mathrm{Al} 203 \\
\mathrm{Fe} 203 \\
\mathrm{MnO} \\
\mathrm{Mg0} \\
\mathrm{CaO} \\
\mathrm{Na20} \\
\mathrm{K} 20 \\
\mathrm{P} 205 \\
\text { LOI }\end{array}$ & $\begin{array}{c}1279 \\
74.0 \\
0.10 \\
12.4 \\
1.07 \\
0.08 \\
0.31 \\
0.66 \\
3.40 \\
4.00 \\
0.01 \\
3.79\end{array}$ & $\begin{array}{c}1323 \\
69.1 \\
0.11 \\
13.4 \\
1.13 \\
0.05 \\
0.94 \\
3.22 \\
1.23 \\
2.64 \\
0.01 \\
8.90\end{array}$ & $\begin{array}{c}1381 \\
72.9 \\
0.09 \\
11.3 \\
1.04 \\
0.05 \\
0.49 \\
3.09 \\
1.64 \\
1.88 \\
0.02 \\
8.79\end{array}$ & $\begin{array}{c}1505 \\
73.2 \\
0.09 \\
12.1 \\
1.02 \\
0.03 \\
0.20 \\
2.46 \\
1.29 \\
3.32 \\
0.00 \\
6.70\end{array}$ & $\begin{array}{c}1667 \\
72.1 \\
0.08 \\
11.3 \\
1.00 \\
0.05 \\
0.12 \\
2.67 \\
1.47 \\
3.47 \\
0.02 \\
7.68\end{array}$ & $\begin{array}{c}2087 \\
71.3 \\
0.16 \\
12.6 \\
1.67 \\
0.08 \\
0.54 \\
1.93 \\
1.92 \\
4.35 \\
0.03 \\
5.16\end{array}$ & $\begin{array}{c}2304 \\
70.9 \\
0.13 \\
12.3 \\
1.55 \\
0.05 \\
0.41 \\
2.62 \\
1.69 \\
3.46 \\
0.03 \\
6.58\end{array}$ \\
\hline Total & 99.8 & 100.7 & 101.3 & 100.4 & 99.9 & 99.7 & 99.8 \\
\hline $\begin{array}{l}\mathrm{Si} \\
\mathrm{Ti} \\
\mathrm{Al} \\
\mathrm{Fe}+3 \\
\mathrm{Mn} \\
\mathrm{Mg} \\
\mathrm{Ca} \\
\mathrm{Na} \\
\mathrm{K} \\
\mathrm{P}\end{array}$ & $\begin{array}{c}72.2 \\
0.07 \\
14.3 \\
0.79 \\
0.05 \\
0.45 \\
0.69 \\
6.43 \\
4.98 \\
0.01\end{array}$ & $\begin{array}{c}71.6 \\
0.09 \\
16.3 \\
0.88 \\
0.04 \\
1.45 \\
3.58 \\
2.47 \\
3.49 \\
0.01\end{array}$ & $\begin{array}{c}\text { ation } \\
75.3 \\
0.07 \\
13.8 \\
0.81 \\
0.04 \\
0.75 \\
3.42 \\
3.28 \\
2.48 \\
0.02\end{array}$ & $\begin{array}{c}\text { cent } \\
74.7 \\
0.07 \\
14.5 \\
0.78 \\
0.02 \\
0.30 \\
2.69 \\
2.55 \\
4.32 \\
0.00\end{array}$ & $\begin{array}{c}74.7 \\
0.06 \\
13.7 \\
0.78 \\
0.04 \\
0.19 \\
2.96 \\
2.95 \\
4.59 \\
0.02\end{array}$ & $\begin{array}{c}71.5 \\
0.12 \\
14.8 \\
1.26 \\
0.06 \\
0.81 \\
2.07 \\
3.73 \\
5.56 \\
0.03\end{array}$ & $\begin{array}{c}72.5 \\
0.10 \\
14.8 \\
1.19 \\
0.04 \\
0.62 \\
2.87 \\
3.35 \\
4.51 \\
0.03\end{array}$ \\
\hline $\begin{array}{l}\mathrm{Ca}+\mathrm{Mg} \\
\mathrm{K} \\
\mathrm{Na}\end{array}$ & $\begin{array}{r}9.1 \\
39.7 \\
51.2\end{array}$ & $\begin{array}{l}45.7 \\
31.8 \\
22.5\end{array}$ & $\begin{array}{l}42.0 \\
24.9 \\
33.1\end{array}$ & $\begin{array}{l}30.3 \\
43.8 \\
25.9\end{array}$ & $\begin{array}{l}29.5 \\
42.9 \\
27.6\end{array}$ & $\begin{array}{l}23.7 \\
45.7 \\
30.7\end{array}$ & $\begin{array}{l}30.8 \\
39.7 \\
29.5\end{array}$ \\
\hline
\end{tabular}

Sample Descriotions:

33. Topopah Spring Member, Paintbrush Tuff. Vitric basal vitrophyre.

34. Topopah Spring Member, Paintbrush Tuff. Clinoptilolite-bearing nonwelded tuff in diagenetic Zone II.

35. Tuff of Calico Hills. Clinoptilolite-bearing nonwelded tuff in diagenetic Zone II.

36. Tuff of Calico Hills. Clinoptilolite-bearing nonwelded tuff in diagenetic Zone II.

37. Tuff of Calico Hills. Clinoptilolite-bearing nonwelded tuff in diagenetic Zone II.

38. Prow Pass Member, Crater Flat Tuff. Clinoptilolite-bearing nonwelded tuff in diagenetic Zone II.

39. Bull frog Member, Crater Flat Tuff. Clinoptilolite- and mordenite(?)-bearing nonwelded tuff in diagenetic Zone II. 
Dri11 Hole UE-25b\#1(H)

Sample

Number

Depth

40.

41.

42.

43.

44.

45.

46.

SiO2

(ft) 2832

2879

$3393 \quad 3602$

3767

3835

3963

$\mathrm{Ti} 02$

$\mathrm{A} 1203$
$\mathrm{Fe} 203$

73.0

67.3

67.1

66.3

68.6

69.3

69.6

0.12

0.25

0.37

0.53

0.48

0.47

14.3

12.8

13.8

0.34

12.9

1.82

2.65

3.66

3.25

3.21

13.8

$\mathrm{MnO}$

0.13

0.04

0.12

0.12

0.12

0.08

2.16

$\mathrm{MgO}$

0.69

1.30

0.83

0.77

1.01

0.03

$\mathrm{CaO}$

2.46

3.13

3.04

2.78

2.51

2.02

1.06

$\mathrm{Na} 2 \mathrm{O}$

$\mathrm{K} 20$

2.07

1.18

2.88

2.80

2.28

2.67

1.73

3.50

3.60

3.51

3.34

2.49

P205

0.03

0.09

0.11

0.18

0.16

0.17

3.18

LOI

Total

4.53

4.43

4.10

3.63

3.34

0.07

$\overline{98.3} \quad \overline{99.2}$

Cation Percent

Si

72.4

67.2

65.8

0.19

0.28

0.40

A1

15.1

$\mathrm{Fe}+3$

0.98

16.0

15.8

16.8

1.41

2.00

2.73

0.08

0.08

2.00

1.14

1.23

3.27

2.96

98.0

99.4

4.98

1.02

3.46

5.60

5.39

4.94

4.48

4.56

0.08

0.09

0.15

32.0

$\mathrm{Ca}+\mathrm{Mg}$

42.8

30.4

29.6

69.0

68.2

69.5

38.7

30.9

32.3

0.36

0.35

0.26

15.1

16.0

16.2

2.46

2.38

1.62

0.08

0.05

0.02

1.16

1.48

1.58

2.71

2.13

1.85

4.45

5.09

4.82

4.51

4.19

4.05

0.14

0.14

0.06

18.5

38.7

38.1

30.1

28.0

27.9

35.2

32.5

32.9

$34.7 \quad 39.5$

39.2

Sample Descriptions:

40. Bull frog Member, Crater Flat Tuff. Mordenite- and clinoptilolitebearing nonwelded tuff in diagenetic Zone II.

41. Bull frog Member, Crater Flat Tuff. Mordenite- and clinoptilolitebearing nonwelded tuff in diagenetic Zone II.

42. Tram Member, Crater Flat Tuff. Analcime- and calcite-bearing nonwelded tuff in diagenetic Zone III.

43. Tram Member, Crater Flat Tuff. Analcime- and calcite-bearing nonwelded tuff in diagenetic Zone III.

44. Tram Member, Crater Flat Tuff. Analcime-, calcite-, and kaolinite-bearing nonwelded tuff in diagenetic Zone III.

45. Tram Member, Crater Flat Tuff. Analcime- and calcite-bearing nonwelded tuff in diagenetic Zone III.

46. Tuff of Lithic Ridge. Analçime- and calcite-bearing nonwelded tuff in diagenetic Zone III. 
Dri11 Hole UE-25p\#1 Cuttings

\begin{tabular}{|c|c|c|c|c|c|c|c|c|}
\hline $\begin{array}{l}\text { Sample } \\
\text { Number }\end{array}$ & 47 . & 48. & 49. & 50. & 51. & 52. & 53. & 54. \\
\hline $\operatorname{Depth}(\mathrm{ft})$ & $\begin{array}{l}1700- \\
1710\end{array}$ & $\begin{array}{l}1730- \\
1740\end{array}$ & $\begin{array}{l}1790- \\
1800\end{array}$ & $\begin{array}{l}2280- \\
2290\end{array}$ & $\begin{array}{l}2650- \\
2660\end{array}$ & $\begin{array}{l}2760- \\
2770\end{array}$ & $\begin{array}{l}3430- \\
3440\end{array}$ & $\begin{array}{l}3560- \\
3570\end{array}$ \\
\hline $\begin{array}{l}\mathrm{SiO} 2 \\
\mathrm{TiO} 2 \\
\mathrm{Al203} \\
\mathrm{Fe} 203 \\
\mathrm{MnC} \\
\mathrm{MgO} \\
\mathrm{CaO} \\
\mathrm{Na2O} \\
\mathrm{K} 2 \mathrm{O} \\
\mathrm{P} 205 \\
\mathrm{LOI}\end{array}$ & $\begin{array}{c}73.2 \\
0.11 \\
12.3 \\
1.36 \\
0.05 \\
0.36 \\
2.37 \\
2.38 \\
2.70 \\
0.02 \\
6.83\end{array}$ & $\begin{array}{c}73.3 \\
0.12 \\
12.7 \\
1.43 \\
0.09 \\
0.30 \\
2.10 \\
2.40 \\
2.91 \\
0.02 \\
6.15\end{array}$ & $\begin{array}{c}72.6 \\
0.14 \\
12.4 \\
1.57 \\
0.06 \\
0.44 \\
2.17 \\
2.25 \\
2.28 \\
0.03 \\
5.34\end{array}$ & $\begin{array}{c}72.7 \\
.0 .17 \\
12.2 \\
1.44 \\
0.10 \\
0.40 \\
2.07 \\
2.25 \\
4.25 \\
0.03 \\
4.11\end{array}$ & $\begin{array}{c}71.5 \\
0.32 \\
12.9 \\
2.28 \\
0.11 \\
1.10 \\
1.73 \\
2.72 \\
4.43 \\
0.10 \\
2.74\end{array}$ & $\begin{array}{c}72.7 \\
0.28 \\
12.8 \\
2.29 \\
0.11 \\
0.92 \\
1.93 \\
2.00 \\
3.34 \\
0.03 \\
3.23\end{array}$ & $\begin{array}{c}74.3 \\
0.17 \\
12.6 \\
1.23 \\
0.05 \\
0.29 \\
0.81 \\
3.00 \\
5.22 \\
0.03 \\
1.99\end{array}$ & $\begin{array}{c}75.1 \\
0.1 \\
11.9 \\
1.34 \\
0.05 \\
0.50 \\
1.48 \\
1.77 \\
5.07 \\
0.03 \\
1.78\end{array}$ \\
\hline Total & 101.6 & 101.5 & 99.3 & 99.8 & 99.9 & 99.6 & 99.7 & 99.2 \\
\hline $\begin{array}{l}\mathrm{Si} \\
\mathrm{Ti} \\
\mathrm{Al} \\
\mathrm{Fe}+3 \\
\mathrm{Mn} \\
\mathrm{Mg} \\
\mathrm{Ca} \\
\mathrm{Na} \\
\mathrm{K} \\
\mathrm{P} \\
\mathrm{Ca}+\mathrm{Mg} \\
\mathrm{K} \\
\mathrm{Na}\end{array}$ & $\begin{array}{c}73.2 \\
0.08 \\
14.5 \\
1.02 \\
0.03 \\
0.54 \\
2.54 \\
4.62 \\
3.45 \\
0.02 \\
27.6 \\
30.9 \\
41.4\end{array}$ & $\begin{array}{c}72.9 \\
0.09 \\
14.9 \\
1.07 \\
0.06 \\
0.44 \\
2.24 \\
4.63 \\
3.69 \\
0.02 \\
24.4 \\
33.6 \\
42.1\end{array}$ & $\begin{array}{c}\quad C a \\
73.5 \\
0.11 \\
14.8 \\
1.20 \\
0.04 \\
0.66 \\
2.35 \\
4.41 \\
2.94 \\
0.03 \\
29.1 \\
28.4 \\
42.6\end{array}$ & $\begin{array}{c}\text { Perc } \\
72.0 \\
0.13 \\
14.3 \\
1.07 \\
0.07 \\
0.59 \\
2.20 \\
4.32 \\
5.37 \\
0.03 \\
22.3 \\
43.0 \\
34.6\end{array}$ & $\begin{array}{c}69.3 \\
0.23 \\
14.7 \\
1.66 \\
0.07 \\
1.59 \\
1.80 \\
5.11 \\
5.48 \\
0.08 \\
24.2 \\
39.2 \\
36.6\end{array}$ & $\begin{array}{c}71.7 \\
0.21 \\
14.9 \\
1.70 \\
0.07 \\
1.35 \\
2.04 \\
3.82 \\
4.20 \\
0.03 \\
29.7 \\
36.8 \\
33.5\end{array}$ & $\begin{array}{c}71.4 \\
0.12 \\
14.3 \\
0.89 \\
0.03 \\
0.42 \\
0.83 \\
5.59 \\
6.40 \\
0.02 \\
9.4 \\
48.3 \\
42.2\end{array}$ & $\begin{array}{c}73.2 \\
0.13 \\
13.7 \\
0.98 \\
0.03 \\
0.73 \\
1.55 \\
3.35 \\
6.30 \\
0.02 \\
19.1 \\
52.9 \\
28.1\end{array}$ \\
\hline
\end{tabular}

Sample Descriptions:

47. Prow Pass Member, Crater Flat Tuff. Clinoptilolite-bearing nonwelded tuff in diagenetic Zone II.

48. Prow Pass Member, Crater Flat Tuff. Clinoptilolite-bearing nonwelded tuff in diagenetic Zone II.

49. Prow Pass Member, Crater Flat Tuff. Clinoptilolite-bearing nonwelded tuff in diagenetic Zone II.

50. Bull frog Member. Crater Flat Tuff. Analcime-bearing nonwelded tuff in diagenetic Zone III.

51. Bull frog Member, Crater Flat Tuff. Analcime-bearing nonwelded tuff in diagenetic Zone III.

52. Bull frog Member, Crater Flat Tuff. Analcime-bearing nonwelded tuff in diagenetic Zone III.

53. Older Tuff Sequence. Authigenic albite-bearing nonwelded tuff in diagenetic Zone IV.

54. 0lder Tuff Sequence. Authigenic albite-bearing nonwelded tuff in diagenetic Zone IV. 
APPENDIX A (cont)

Drill Hole J-13

\begin{tabular}{|c|c|c|c|c|c|c|c|}
\hline $\begin{array}{l}\text { Sample } \\
\text { Number }\end{array}$ & 55. & 56. & 57. & 58. & 59. & 60. & 61. \\
\hline Depth (ft) & 1457 & 1519 & 1883 & 2001 & 21.33 & 2982 & 2999 \\
\hline $\begin{array}{l}\mathrm{SiO} 2 \\
\mathrm{Ti02} \\
\mathrm{A} 1203 \\
\mathrm{Fe} 203 \\
\mathrm{MnO} \\
\mathrm{MgO} \\
\mathrm{CaO} \\
\mathrm{Na} 20 \\
\mathrm{~K} 20 \\
\mathrm{P} 205 \\
\mathrm{LOI}\end{array}$ & $\begin{array}{c}69.6 \\
0.08 \\
10.6 \\
0.94 \\
0.05 \\
0.50 \\
1.96 \\
0.88 \\
3.55 \\
0.02 \\
10.14\end{array}$ & $\begin{array}{c}69.5 \\
0.08 \\
12.1 \\
1.01 \\
0.04 \\
0.39 \\
1.87 \\
1.22 \\
3.94 \\
0.02 \\
8.65\end{array}$ & $\begin{array}{c}75.2 \\
0.11 \\
12.6 \\
1.44 \\
0.10 \\
0.11 \\
0.68 \\
3.91 \\
4.39 \\
0.02 \\
0.77\end{array}$ & $\begin{array}{c}70.1 \\
0.19 \\
13.9 \\
1.67 \\
0.02 \\
0.14 \\
0.41 \\
5.55 \\
2.94 \\
0.02 \\
3.74\end{array}$ & $\begin{array}{c}75.3 \\
0.11 \\
12.6 \\
1.44 \\
0.09 \\
0.23 \\
0.75 \\
4.02 \\
4.38 \\
0.03 \\
0.30\end{array}$ & $\begin{array}{c}74.8 \\
0.16 \\
12.2 \\
1.41 \\
0.05 \\
0.21 \\
0.89 \\
3.70 \\
4.46 \\
0.02 \\
0.94\end{array}$ & $\begin{array}{c}70.0 \\
0.30 \\
12.4 \\
2.18 \\
0.10 \\
0.51 \\
2.52 \\
2.55 \\
4.18 \\
0.10 \\
3.81\end{array}$ \\
\hline Total & 98.3 & 98.9 & 99.3 & 98.7 & 99.3 & 98.8 & 98.6 \\
\hline $\begin{array}{l}\mathrm{Si} \\
\mathrm{Ti} \\
\mathrm{Al} \\
\mathrm{Fe}+3 \\
\mathrm{Mn} \\
\mathrm{Mg} \\
\mathrm{Ca} \\
\mathrm{Na} \\
\mathrm{K} \\
\mathrm{P} \\
\mathrm{Ca}+\mathrm{Mg} \\
\mathrm{K} \\
\mathrm{Na}\end{array}$ & $\begin{array}{c}75.7 \\
0.07 \\
13.6 \\
0.77 \\
0.04 \\
0.81 \\
2.28 \\
1.85 \\
4.92 \\
0.02 \\
31.3 \\
49.9 \\
18.8\end{array}$ & $\begin{array}{c}73.4 \\
0.06 \\
15.1 \\
0.80 \\
0.03 \\
0.61 \\
2.12 \\
2.50 \\
5.31 \\
0.02 \\
25.9 \\
50.4 \\
23.7\end{array}$ & $\begin{array}{c}\text { Cation } \\
71.4 \\
0.08 \\
14.1 \\
1.03 \\
0.07 \\
0.16 \\
0.69 \\
7.19 \\
5.31 \\
0.02 \\
6.3 \\
39.8 \\
53.9\end{array}$ & $\begin{array}{c}\text { Percent } \\
68.0 \\
0.14 \\
15.9 \\
1.22 \\
0.01 \\
0.20 \\
0.43 \\
10.44 \\
3.64 \\
0.0 ? \\
4.3 \\
24.7 \\
71.0\end{array}$ & $\begin{array}{c}71.1 \\
0.08 \\
14.0 \\
1.02 \\
0.06 \\
0.32 \\
0.76 \\
7.36 \\
5.27 \\
0.02 \\
7.9 \\
38.5 \\
53.6\end{array}$ & $\begin{array}{c}71.5 \\
0.12 \\
13.8 \\
1.02 \\
0.03 \\
0.30 \\
0.91 \\
6.86 \\
5.44 \\
0.02 \\
9.0 \\
40.3 \\
50.8\end{array}$ & $\begin{array}{c}69.8 \\
0.22 \\
14.5 \\
1.63 \\
0.07 \\
0.76 \\
2.69 \\
4.93 \\
5.31 \\
0.08 \\
25.2 \\
38.8 \\
36.0\end{array}$ \\
\hline
\end{tabular}

Sample Descriptions:

55. Tuff of Calico Hills. Clinoptilolite-bearing nonwelded tuff in Zone II.

56. Tuff of Calico Hills. Clinoptilolite-bearing nonwelded tuff in diagenetic Zone II.

57. Prow Pass Member, Crater Flat Tuff. Devitrified partially welded tuff.

58. Prow Pass Member, Crater Flat Tuff. Analcime-bearing nonwelded tuff in diagenetic Zone III.

59. Bull frog Member, Crater Flat Tuff. Devitrified partially welded tuff.

60. Tram Member, Crater Flat Tuff. Authigenic albite-bearing nonwelded tuff in diagenetic Zone IV.

61. Tram Member, Crater Flat Tuff. Analcime- and authigenic zlbite-bearing nonwelded tuff in diagenetic Zone IV. 


\section{APPENDIX A (cont)}

Outcrop Samples from Prow Pass, Northern Yucca Mountain ${ }^{\text {a }}$

Sample

Number

Field

Number

Si02

$\mathrm{Ti} 02$

A1 203

$\mathrm{Fe} 203$

$\mathrm{FeO}$

$\mathrm{MnO}$

$\mathrm{MgO}$

$\mathrm{CaO}$

$\mathrm{Na} 20$

$\mathrm{K} 20$

P205

LOI

Total

Si

Ti

A1

$\mathrm{Fe}+3$

$\mathrm{Fe}+2$

$\mathrm{Mn}$

$\mathrm{Mg}$

$\mathrm{Ca}$

$\mathrm{Na}$

K

$P$

$\mathrm{Ca}+\mathrm{Mg}$

$K$

$\mathrm{Na}$
62.

63.

$$
3-15-82-5
$$

69.5

0.08

12.3

0.75

0.07

0.04

0.10

1.08

0.68

6.57

0.01

8.42

99.7

Cation Percent

$$
72.6
$$

0.06

15.2

0.59

0.06

0.03

0.16

1.21

1.38

8.75

0.01

11.9

76.2

12.0
$3-15-82-9$

66.6

$0.1 \mathrm{~J}$

12.4

1.05

0.04

0.04

0.15

0.61

0.59

7.81

0.01

10.04

99.5

Sample Descriptions:

62. Tuff of Calico Hills. Clinoptilolite- and mordenite-bearing nonwelded tuff in Zone II. Nevada State Coordinates $786500 \mathrm{~N}, 551000 \mathrm{E}$.

63. Tuff of Calico Hills. Clinoptilo?ite- and mordenite-bearing nonwelded tuff in Zone II. Nevada State Coordinates 786700 N, 551400E.

a

These samples analyzed by atomic absorption spectrophotometry; J. Husler, analyst. 
APPENDIX B

COMPARISON OF ANALYSES DETERMINED BY X-RAY FLUORESCENCE (XRF), ATOMIC ABSORPTION (AA), AND EMISSION SPECTROPHOTOMETRY (ES) FOR DRILL CORE SAMPLES FROM YUCCA MOUNTAIN, NEVADA

Drill Hole USW GU-3 and USW G-3

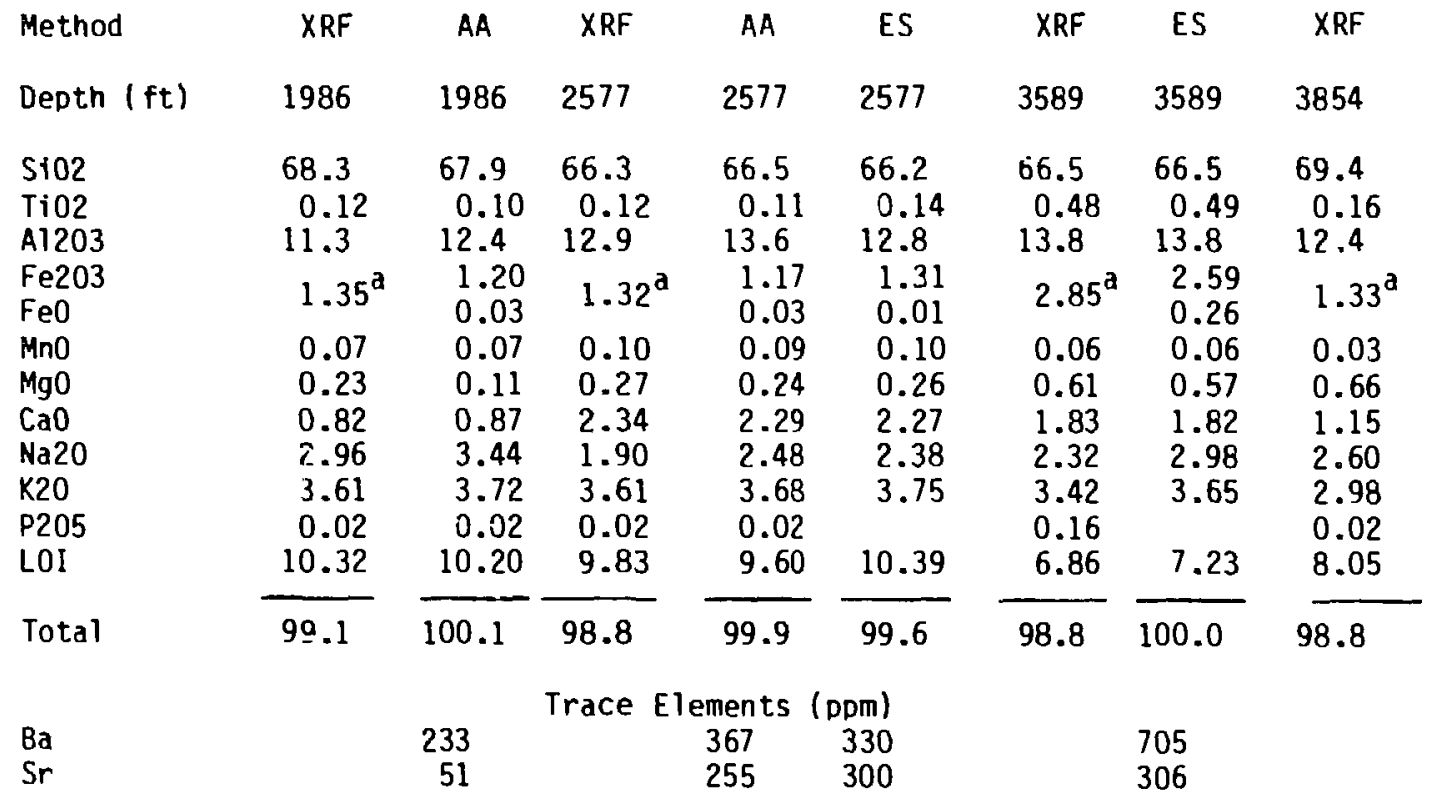

Drill Hole USW G-3

\begin{tabular}{|c|c|c|c|}
\hline Me thod & ES & XRF & ES \\
\hline Depth $(f t)$ & 3854 & 4869 & 4869 \\
\hline $\begin{array}{l}\text { SiO2 } \\
\text { Ti02 }\end{array}$ & $\begin{array}{c}69.2 \\
0.16\end{array}$ & $\begin{array}{c}71.0 \\
0.23\end{array}$ & $\begin{array}{r}71.7 \\
0.23\end{array}$ \\
\hline Al203 & 12.6 & 12.9 & 13.1 \\
\hline $\begin{array}{l}\mathrm{Fe} 203 \\
\mathrm{Fe} 0\end{array}$ & $\begin{array}{l}0.81 \\
0.46\end{array}$ & 1.58 & $\begin{array}{l}0.97 \\
0.57\end{array}$ \\
\hline MnO & 0.02 & 0.05 & 0.06 \\
\hline $\mathrm{MgO}$ & 0.49 & 0.50 & 0.50 \\
\hline CaO & 1.10 & 0.96 & 1.04 \\
\hline $\mathrm{Na} 20$ & 3.32 & 3.62 & 4.70 \\
\hline $\begin{array}{l}\text { K20 } \\
\text { P205 }\end{array}$ & 3.18 & $\begin{array}{l}2.57 \\
0.04\end{array}$ & 2.68 \\
\hline LOI & 8.40 & 4.11 & 4.26 \\
\hline Total & 99.8 & 97.5 & 99.9 \\
\hline Trac & $\begin{array}{r}\text { Eleme } \\
1130 \\
240\end{array}$ & $s(p p m)$ & $\begin{array}{l}520 \\
200\end{array}$ \\
\hline
\end{tabular}

${ }^{\mathrm{a}}$ Total iron, reported as $\mathrm{Fe}_{2} \mathrm{O}_{3}$. 
APPENDIX B (cont)

Drill Hole USW G-4

\begin{tabular}{|c|c|c|c|c|c|c|c|c|}
\hline Method & $X R F$ & $A A$ & ES & XRF & AA & ES & XRF & ES \\
\hline Depth $(\mathrm{ft})$ & 1470 & 1470 & 1470 & 1544 & 1544 & 1544 & 2131 & 2131 \\
\hline $\mathrm{SiO}$ & 69.5 & 69.1 & 69.2 & 69.3 & 69.0 & 69.1 & 65.1 & 65.7 \\
\hline Ti02 & 0.07 & 0.09 & 0.08 & 0.07 & 0.07 & 0.06 & 0.11 & 0.12 \\
\hline A1 203 & 10.9 & 11.6 & 11.2 & 11.3 & 11.9 & 11.5 & 13.0 & 13.2 \\
\hline $\begin{array}{l}\mathrm{Fe} 203 \\
\mathrm{FeO}\end{array}$ & $0.90^{\mathrm{a}}$ & $\begin{array}{l}0.74 \\
0.01\end{array}$ & $\begin{array}{l}0.72 \\
0.07\end{array}$ & $0.96^{\mathrm{a}}$ & $\begin{array}{l}0.77 \\
0.02\end{array}$ & $\begin{array}{l}0.81 \\
0.04\end{array}$ & $1.33^{\mathrm{a}}$ & $\begin{array}{l}1.12 \\
0.12\end{array}$ \\
\hline Mn0 & 0.05 & 0.05 & 0.05 & 0.04 & 0.03 & 0.03 & 0.02 & 0.02 \\
\hline Mg0 & 0.05 & 0.04 & 0.03 & 0.06 & 0.04 & 0.03 & 0.17 & 0.15 \\
\hline $\mathrm{CaO}$ & 0.89 & 0.88 & 0.88 & 0.84 & 0.68 & 0.75 & 2.62 & 2.63 \\
\hline $\begin{array}{l}\mathrm{Na20} \\
\text { K20 }\end{array}$ & $\begin{array}{l}2.18 \\
4.31\end{array}$ & $\begin{array}{l}2.54 \\
4.50\end{array}$ & $\begin{array}{l}2.53 \\
4.40\end{array}$ & $\begin{array}{l}2.38 \\
4.00\end{array}$ & $\begin{array}{l}2.87 \\
4.13\end{array}$ & $\begin{array}{l}2.82 \\
4.10\end{array}$ & 1.96 & $\begin{array}{l}2.46 \\
3.52\end{array}$ \\
\hline P205 & 0.02 & 0.01 & & 0.02 & 0.01 & & 0.01 & \\
\hline LOI & 10.10 & 10.15 & 10.82 & 9.90 & 9.79 & 10.56 & 10.56 & 11.08 \\
\hline Total & 98.9 & 99.7 & 100.0 & 98.9 & 99.4 & 99.8 & 98.3 & 100.1 \\
\hline & & & Trace & Elements & $(\mathrm{ppm})$ & & & \\
\hline $\begin{array}{l}\mathrm{Ba} \\
\mathrm{Sr}\end{array}$ & & $\begin{array}{l}90 \\
25\end{array}$ & $\begin{array}{l}80 \\
30\end{array}$ & & $\begin{array}{l}81 \\
25\end{array}$ & $\begin{array}{l}60 \\
30\end{array}$ & & $\begin{array}{r}170 \\
64\end{array}$ \\
\hline
\end{tabular}

Drill Hole USW G-4

$\begin{array}{lllllll}\text { Method XRF } & \text { AA } & \text { XRF } & \text { AA } & \text { ES } & \text { XRF } & \text { AA }\end{array}$

$\begin{array}{llllllll}\text { Depth (ft) } & 2226 & 2226 & 2716 & 2716 & 2716 & 2792 & 2792\end{array}$

\begin{tabular}{|c|c|c|c|c|c|c|c|}
\hline $\begin{array}{l}\text { Si02 } \\
\text { Ti02 } \\
\text { A1203 }\end{array}$ & $\begin{array}{c}66.4 \\
0.14 \\
13.1\end{array}$ & $\begin{array}{c}66.7 \\
0.17 \\
13.4\end{array}$ & $\begin{array}{c}67.7 \\
0.13 \\
12.2\end{array}$ & $\begin{array}{c}67.8 \\
0.12 \\
13.0\end{array}$ & $\begin{array}{c}67.8 \\
0.13 \\
12.5\end{array}$ & $\begin{array}{c}68.3 \\
0.16 \\
11.6\end{array}$ & $\begin{array}{c}67.9 \\
0.15 \\
12.4\end{array}$ \\
\hline $\begin{array}{l}\mathrm{Fe} 203 \\
\mathrm{Fe} 0\end{array}$ & $1.45^{\mathrm{a}}$ & $\begin{array}{l}1.33 \\
0.00\end{array}$ & $1.24^{\mathrm{a}}$ & $\begin{array}{l}0.98 \\
0.07\end{array}$ & $\begin{array}{l}1.29 \\
0.14\end{array}$ & $1.32^{\mathrm{a}}$ & $\begin{array}{l}1.04 \\
0.13\end{array}$ \\
\hline MnO & 0.05 & 0.04 & 0.04 & 0.04 & 0.04 & 0.03 & 0.03 \\
\hline MgO & 0.25 & 0.22 & 0.20 & 0.11 & 0.19 & 0.24 & 0.18 \\
\hline $\mathrm{CaO}$ & 2.43 & 2.46 & 2.57 & 2.60 & 2.70 & 2.94 & 2.93 \\
\hline $\mathrm{Na} 20$ & 1.65 & 2.15 & 2.18 & 2.81 & 2.82 & 2.03 & 2.68 \\
\hline $\mathrm{K} 20$ & 3.78 & 3.92 & 2.40 & 2.47 & 2.55 & 1.63 & 1.68 \\
\hline P205 & 0.01 & 0.02 & 0.01 & 0.02 & & 0.02 & 0.03 \\
\hline LOI & 9.40 & 9.27 & 10.11 & 10.08 & 10.20 & 10.98 & 10.93 \\
\hline Total & 98.7 & 99.7 & 98.8 & 100.2 & 100.4 & 99.3 & 100.1 \\
\hline & \multicolumn{7}{|c|}{ Trace Elements (ppm) } \\
\hline $\mathrm{Ba}$ & & 269 & & 349 & 350 & & 564 \\
\hline Sr & & 153 & & 102 & 122 & & 170 \\
\hline
\end{tabular}




\section{APPENDIX B (cont)}

\section{Drill Hole J-13}

\begin{tabular}{|c|c|c|c|c|c|c|c|}
\hline Method & XRF & $A A$ & XRF & $A A$ & ES & XRF & $\mathrm{AA}$ \\
\hline Depth ( ft) & 1457 & 1457 & 1519 & 1519 & 1519 & 2001 & 2001 \\
\hline $\begin{array}{l}\mathrm{SiO} 2 \\
\mathrm{TiO} \\
\mathrm{Al} 203 \\
\mathrm{Fe} 203 \\
\mathrm{FeO}\end{array}$ & $\begin{array}{c}69.6 \\
0.08 \\
10.6 \\
0.94^{\mathrm{a}}\end{array}$ & $\begin{array}{c}70.3 \\
0.08 \\
11.3 \\
0.70 \\
0.08\end{array}$ & $\begin{array}{c}69.5 \\
0.08 \\
12.1 \\
1.01^{\mathrm{a}}\end{array}$ & $\begin{array}{c}70.0 \\
0.09 \\
12.8 \\
0.83 \\
0.04\end{array}$ & $\begin{array}{c}70.0 \\
0.08 \\
12.2 \\
0.74 \\
0.14\end{array}$ & $\begin{array}{c}70.1 \\
0.19 \\
13.9 \\
1.67^{\mathrm{a}}\end{array}$ & $\begin{array}{c}70.5 \\
0.19 \\
14.8 \\
1.53 \\
0.03\end{array}$ \\
\hline $\begin{array}{l}\mathrm{MnO} \\
\mathrm{MgO} \\
\mathrm{CaO} \\
\mathrm{Na} 20 \\
\mathrm{~K} 20 \\
\mathrm{P} 205 \\
\mathrm{LOI}\end{array}$ & $\begin{array}{r}0.05 \\
0.50 \\
1.96 \\
0.77 \\
3.55 \\
0.02 \\
10.14\end{array}$ & $\begin{array}{r}0.06 \\
0.52 \\
1.93 \\
0.88 \\
3.68 \\
0.01 \\
10.20\end{array}$ & $\begin{array}{l}0.04 \\
0.39 \\
1.87 \\
1.04 \\
3.94 \\
0.02 \\
8.65\end{array}$ & $\begin{array}{l}0.05 \\
0.38 \\
1.86 \\
1.26 \\
4.14 \\
0.01 \\
8.58\end{array}$ & $\begin{array}{l}0.04 \\
0.37 \\
1.86 \\
1.18 \\
4.13\end{array}$ & $\begin{array}{l}0.02 \\
0.14 \\
0.41 \\
4.88 \\
2.94 \\
0.02 \\
3.74\end{array}$ & $\begin{array}{l}0.02 \\
0.18 \\
0.38 \\
5.55 \\
3.02 \\
0.02 \\
3.92\end{array}$ \\
\hline Total & 98.3 & 99.7 & 98.7 & 100.1 & 100.0 & 98.0 & 100.5 \\
\hline $\begin{array}{l}\text { Ba } \\
\text { Sr }\end{array}$ & & $\begin{array}{l}72 \\
17\end{array}$ & & $\begin{array}{l}\text { Eleme } \\
134 \\
51\end{array}$ & $\begin{array}{c}(\mathrm{ppm}) \\
120 \\
60\end{array}$ & & $\begin{array}{r}340 \\
68\end{array}$ \\
\hline
\end{tabular}

\begin{tabular}{lcc}
\multicolumn{3}{l}{ Drill Hole UE-25b/1(H) } \\
\cline { 1 - 2 } Method & XRF & ES \\
Depth (ft) & 2879 & 2879 \\
& & \\
Si02 & 67.3 & 66.9 \\
Ti02 & 0.25 & 0.26 \\
A1203 & 13.2 & 13.2 \\
Fe203 & 1.82 & 1.48 \\
Fe0 & 0.04 & 0.24 \\
Mn0 & 1.30 & 1.32 \\
Mg0 & 3.13 & 3.24 \\
Ca0 & 1.05 & 1.18 \\
Na20 & 3.75 & 3.90 \\
K20 & 0.09 & \\
P205 & 7.24 & 7.86 \\
L0I & & \\
Total & 99.1 & 99.7 \\
& & \\
Trace Elements & (ppm) & \\
Ba & & 1040 \\
Sr & & 1060
\end{tabular}




\section{APPENDIX C}

MAJOR- AND TRACE-ELEMENT CONCENTRATIONS DETERMINED BY NEUTRON ACTIVATION ANALYSIS IN WHOLE-ROCK SAMPLES FROM DRILL CORE IN DRILL HOLES USW G-1, USW G-2, USW GU-3, AND FROM TUFF OF CALICO HILLS SAMPLES COLLECTED IN OUTCROP AT PROW PASS, NORTHERN YUCCA MOUNTAIN

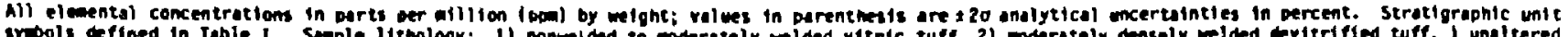

vitrophre, 4l sectice- ind seollte- Ych vitroghrtc tuff, 5)

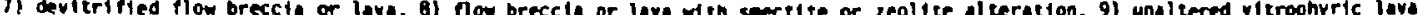

USW G-1

\begin{tabular}{|c|c|c|c|c|c|c|c|c|c|c|c|c|c|c|}
\hline $\begin{array}{l}\text { Depth } \\
(\mathrm{ft})\end{array}$ & 1191 & 1240 & 1286 & 1319 & 1341 & 1351 & 1392 & 1436 & 1492 & 1561 & 1639 & 1693 & 1748 & 1714 \\
\hline Unte & TDE & Tpt & Tot & Tot & Tot & TDE & Tpt & The & Tht & The & The & The & The & The \\
\hline $\begin{array}{l}\text { Sample } \\
\text { Lithology }\end{array}$ & $y$ & 2 & 4 & $\cdot 3$ & 3 & 1 & 1 & 6 & 6 & 6 & 6 & 6 & 6 & 6 \\
\hline A! & 68100 & 68400 & 87300 & 66700 & 65800 & 68500 & 63300 & 60800 & 61100 & 61100 & 66100 & $62900^{n}$ & 52300 & 67000 \\
\hline Au & $<0.12$ & $<0.12$ & $<0.12$ & $<0.12$ & $<0.12$ & 0.12 & $<0.12$ & & $<0.12$ & $<0.36$ & 0.12 & $\infty .12$ & 0.12 & 0.12 \\
\hline Es & $<360$ & $<380$ & $<290$ & $<400$ & $<380$ & $<390$ & 4350 & & $<300$ & $<390$ & $<330$ & 230 & 160 & 650 \\
\hline Ca & 2800 & 3800 & 16100 & 4000 & 4100 & 5000 & 6200 & 5000 & 3900 & 4900 & 3900 & 4000 & 4800 & 9100 \\
\hline ce & 74 & 74 & 120 & 74 & 71 & 99 & $n$ & 42 & 46 & 85 & 49 & 94 & 45 & 85 \\
\hline $\mathrm{Cl}$ & $<210$ & $<240$ & $<170$ & 570 & 460 & 280 & 290 & & $<230$ & $<240$ & $<220$ & $<180$ & $<220$ & $<240$ \\
\hline Co & $<1.6$ & $<0.6\rangle$ & $<2.1$ & $<0.66$ & $\times 1.8$ & 42.4 & $<0.60$ & & $<0.66$ & $<2.4$ & $<0.74$ & $<1.8$ & 2.1 & 2.0 \\
\hline $\mathrm{Cr}$ & $<13$ & $1.0(60)$ & $<13$ & $<13$ & [13 & $<16$ & $<16$ & $1.2(99)$ & $\{13$ & $: 16$ & $1.0(60)$ & $<13$ & 413 & $<16$ \\
\hline Cs & 4.5 & $3.75(8)$ & 8.5 & $5.8(11)$ & 1.6 & 10.0 & $11.3(9)$ & $8.6(21)$ & $6.7(10)$ & 6.9 & $5.1(10)$ & 6.0 & 5.1 & 4.9 \\
\hline Dy & 4.7 & 4.9 & 5.2 & 5.2 & 5.0 & 4.1 & 6.0 & 3.2 & 4.0 & 5.0 & 3.9 & 6.4 & 1.5 & 4.4 \\
\hline $\mathbb{E} u$ & & $0.30(15)$ & & $0.21(25)$ & & & $0.30(17)$ & $0.11(73)$ & $0.26(17)$ & & $0.22117)$ & & & \\
\hline Fe & 6300 & $6800(10)$ & 8900 & $6300(24)$ & 6200 & 6800 & $7200(20)$ & $5800(13)$ & $5700(23)$ & 6000 & 6900 (11) & 6000 & 6500 & 9900 \\
\hline$H$ & 4.3 & 5.3 & 1.5 & 4.3 & 4.3 & 4.3 & 6.4 & $4.5(22)$ & 4.3 & 3.2 & 5.3 & 3.2 & 3.2 & 3.2 \\
\hline$x$ & 37100 & 36400 & 9030 & 33500 & 32200 & 31100 & 33000 & & 31300 & 34500 & 30600 & 36800 & 23700 & 27300 \\
\hline La & 35 & 41 & 52 & 30 & 32 & 47 & 30 & 24 & 27 & 33 & 31 & 4 & 27 & 37 \\
\hline Lu & 0.37 & 0.48 & 0.61 & 0.37 & 0.37 & 0.37 & 0.48 & & 0.37 & 0.60 & 0.24 & 0.48 & 0.11 & 0.48 \\
\hline M & $<5800$ & 420 & 5600 & 17300 & $<6300$ & 26400 & $<5900$ & $<180$ & $<5900$ & $<6700$ & $<180$ & 44700 & $<5700$ & $<6600$ \\
\hline Mn & 490 & 490 & 500 & 470 & 190 & 520 & 540 & 330 & 196 & 270 & 250 & 104 & 126 & 620 \\
\hline Wa & 22800 & 25900 & 10100 & 26000 & 26100 & 24000 & 20000 & 22900 & 21500 & 22100 & 22500 & 19800 & 19100 & 22400 \\
\hline to & 195 & 184 & $<52$ & 154 & 160 & 187 & 176 & & 193 & 157 & 157 & 194 & 166 & 131 \\
\hline is & 41.5 & $<1.5$ & 4.3 & $<4.3$ & $<1.5$ & $<4.3$ & 4.3 & & $<4.3$ & 4.3 & $<4.3$ & $<1.5$ & 41.5 & 4.3 \\
\hline ce & 2.3 & $2.42(9)$ & 3.4 & $2.34(11)$ & 2.2 & 2.3 & $2.84(9)$ & & $2.33(9)$ & 2.4 & $2.48(9)$ & 2.4 & 2.1 & 2.6 \\
\hline 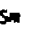 & 5.6 & $6.0(20)$ & $<8.2$ & $<6.7$ & 5.2 & 4.9 & 47.1 & $3.6\{22\}$ & $\ll 5.8$ & 5.8 & $5.6(22)$ & 8.1 & 44.6 & 45.5 \\
\hline 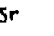 & 679 & 43 & 1650 & 4760 & 4720 & $\$ 720$ & $<660$ & 43 & $<550$ & $<720$ & 54 & $C 420$ & $<510$ & 1800 \\
\hline a & $<1.7$ & $3.1(50)$ & 2.2 & $<1.5$ & $1.1(100)$ & $<5$ & $2.1(48)$ & & $2.55(31)$ & 45 & C1.4 & $1.1(100)$ & $<1.7$ & $<1.7$ \\
\hline$\Delta$ & $<2.3$ & 41.6 & $<2.3$ & 41.6 & $<2.3$ & 2.3 & 4.7 & & 41.7 & $<2.3$ & $<1.7$ & $<2.3$ & $<2.3$ & $<2.3$ \\
\hline n & 24.7 & 25.2 & 35.1 & 22.8 & 24.4 & 27.0 & 27.7 & 22 & 24.9 & 28.0 & 22.8 & 29.2 & 19.5 & 18.1 \\
\hline 1 & $<1700$ & 600 & 860 & $<1900$ & $<1800$ & $<1800$ & 41600 & 450 & $<1400$ & $\times 1800$ & 430 & 410 & $<1300$ & 980 \\
\hline & 4.18 & 4.50 & 1.37 & 4.70 & 4.52 & 4.05 & 3.46 & $4.7(21)$ & 3.69 & 3.87 & 4.01 & 4.48 & 4.28 & 4.59 \\
\hline & $<18$ & $<19$ & $<16$ & 42 & $<19$ & 220 & $<19$ & & $<17$ & $\checkmark 18$ & $<17$ & $<! 3$ & $<15$ & $<21$ \\
\hline$b$ & 2.9 & $3.5(2 y)$ & 3.3 & 3.3 & 2.3 & 2.8 & 3.4 & $1.7(35)$ & 2.3 & 4.5 & $2.6(31)$ & 3.5 & 1.3 & 2.6 \\
\hline$\eta$ & & $37(53)$ & & $37(69)$ & & & $47(48)$ & & $35(69)$ & & $26(99)$ & & & \\
\hline
\end{tabular}




\section{APPENDIX C (cont)}

USW G-1

\begin{tabular}{|c|c|c|c|c|c|c|c|c|c|c|c|c|c|c|}
\hline $\begin{array}{l}\text { Depth } \\
(\mathrm{ft})\end{array}$ & 1820 & 1942 & 1983 & 2041 & 2083 & 2136 & 2173 & 2233 & 2247 & 2291 & 2318 & 2436 & 2486 & 2555 \\
\hline Unit & $T C D$ & $T C D$ & $\operatorname{Tcp}$ & $T C p$ & TCD & $T C D$ & ret & $T c b$ & $T C b$ & $T C D$ & ICb & $T C b$ & $T C D$ & $T C D$ \\
\hline $\begin{array}{l}\text { Samplo: } \\
\text { Lithol ogy }\end{array}$ & 6 & 2 & 2 & 6 & 6 & 6 & 6 & 6 & 6 & 6 & 6 & 2 & 2 & 6 \\
\hline Al. & 66400 & 72700 & 70800 & 65600 & 62700 & 64100 & 73000 & 70300 & 72000 & 67700 & 93600 & 69600 & 72200 & 66700 \\
\hline$A u$ & $<0.12$ & $<0.12$ & & $<0.12$ & $<0.12$ & $<0.12$ & 50.17 & $\therefore 0.12$ & 0.12 & $<0.12$ & $<0.12$ & $<0.12$ & $<0.12$ & $<0.12$ \\
\hline Ba & $<430$ & $<640$ & & $<320$ & 191 & $<430$ & 500 & 560 & 540 & 440 & 700 & 410 & 350 & 420 \\
\hline $\mathrm{Ca}$ & 7000 & $<5200$ & 3400 & 6100 & 5100 & $\$ 100$ & 6400 & 7200 & 7700 & 15000 & 7600 & $<3500$ & 4400 & 9000 \\
\hline $\mathrm{Ce}$ & 87 & 122 & 77 & 66 & 88 & 79 & 138 & 114 & 139 & 109 & 127 & 93 & 98 & 89 \\
\hline $\mathrm{Cl}$ & $<260$ & $<380$ & & $<250$ & $<220$ & $<270$ & $<240$ & $<260$ & $<280$ & $<300$ & $\measuredangle 290$ & $<290$ & $<270$ & $<230$ \\
\hline $\mathrm{CO}$ & 2.0 & $<2.0$ & & $<2.0$ & $<2.0$ & $<2.2$ & $<2.8$ & & $<2.2$ & $<2.8$ & $<0.63$ & $<2.0$ & $<0.60$ & $<2.2$ \\
\hline $\mathrm{Cr}$ & $<18$ & $<15$ & 4.5 & $<15$ & $<15$ & $<18$ & $<18$ & 3.4 & $<15$ & $<15$ & $<15$ & 215 & 3.6 & $<15$ \\
\hline $\mathrm{Cs}$ & 7.0 & 1.6 & 4.0 & 5.1 & 5.4 & 5.4 & 3.6 & 3.7 & 5.3 & 3.4 & $0.66(44)$ & 3.2 & 4.6 & 5.9 \\
\hline Or & 4.7 & 7.9 & 6.6 & 4.1 & 4.4 & 2.7 & 4.6 & 4.8 & 4.0 & 6.9 & 4.8 & 5.1 & 5.3 & 5.3 \\
\hline Eu & & & 0.33 & & & & & 0.51 & & & $0.68(13)$ & & 0.52 & \\
\hline $\mathrm{Fe}$ & 9200 & 8500 & 9500 & 9600 & 8100 & 9400 & 13200 & 9700 & 10400 & 8900 & 11600 & 8900 & 9900 & 7800 \\
\hline Hf & 6.4 & 6.4 & 7.5 & 6.4 & 5.4 & 6.4 & 6.4 & 7.2 & 6.4 & 5.4 & 10.2 & 5.4 & 7.3 & 4.3 \\
\hline$k$ & 29900 & 36400 & & 30400 & 27900 & 26700 & 46900 & 41900 & 36600 & 17700 & 50400 & 37800 & 34000 & 19900 \\
\hline La & 68 & 51 & 51 & 38 & 38 & 34 & 83 & 59 & 70 & 77 & 71 & 48 & 58 & 38 \\
\hline Lu & 0.38 & 0.63 & & 0.38 & 0.51 & 0.25 & 0.38 & 0.45 & 0.38 & 0.51 & 0.36 & 0.63 & 0.63 & 0.51 \\
\hline $\mathrm{Mg}$ & $<6900$ & $<9300$ & 180 & $<6700$ & $<6300$ & $<7200$ & $<6200$ & $<180$ & $<7300$ & $<7600$ & $<7300$ & $<8100$ & $<180$ & $<7400$ \\
\hline Mn & 440 & 1890 & 260 & 125 & 138 & 420 & 274 & 490 & 1040 & 610 & 283 & 840 & 620 & 320 \\
\hline Ma & 22000 & 28600 & 24800 & 25300 & 24300 & 24500 & 20600 & 21000 & 21800 & 25700 & 25900 & 25400 & 29700 & 28000 \\
\hline $\mathrm{RD}$ & 155 & 134 & 148 & 156 & 137 & 159 & 170 & 152 & 190 & 81 & 118 & 187 & 174 & 97 \\
\hline Sb & $<4.3$ & 4.3 & & $<1.5$ & $<1.5$ & $<4.3$ & $<4.3$ & $<4.3$ & $<4.3$ & $<4.3$ & $<4.3$ & $<4.3$ & $<4.3$ & $<1.5$ \\
\hline Sc & 1.7 & 1.8 & & 1.9 & 1.4 & 1.8 & 2.8 & 1.09 & 1.2 & 1.2 & 1.36 & 1.0 & 1.90 & 0.9 \\
\hline Sin & 10.3 & 7.7 & 6.6 & 6.1 & 6.5 & 5.5 & 7.6 & 7.6 & 8.8 & 8.7 & 8.3 & 7.4 & 6.3 & 5.2 \\
\hline$S r$ & $<770$ & $<1300$ & 48 & $<580$ & $<600$ & $<780$ & $<620$ & 120 & $<940$ & $<860$ & $<690$ & 41000 & 100 & $<720$ \\
\hline Ta & $<5$ & $<5$ & & $<5$ & $<5$ & $<5$ & $<5$ & $<3.2$ & $<5$ & $<5$ & $3.0(38)$ & $<1.7$ & 2.2 & $<1.7$ \\
\hline To & $<2.3$ & $<2.3$ & & $<2.3$ & $<2.3$ & $<2.3$ & $<2.3$ & $<2.0$ & $<2.3$ & $<2.3$ & $<1.7$ & $<2.3$ & $<1.5$ & $<2.3$ \\
\hline Th & 23.1 & 21.9 & 22 & 21.3 & 20.5 & 22.2 & 19.2 & 22.1 & 25.5 & 22.7 & 29.3 & 21.9 & 22.5 & 18.6 \\
\hline TI & $<1900$ & $<3100$ & 840 & $<1500$ & 700 & $<1500$ & 1400 & 980 & $<2300$ & 1100 & 1340 & $<2500$ & 880 & $<1800$ \\
\hline u & 4.33 & 4.57 & 3.1 & 2.75 & 3.48 & 2.65 & 2.17 & 3.20 & 3.07 & 3.68 & 4.08 & 4.86 & 4.09 & 3.71 \\
\hline$v$ & $<21$ & $<35$ & & $<18$ & $<16$ & $<21$ & $<18$ & $<22$ & $<25$ & $<24$ & $<22$ & $<26$ & $<22$ & $<21$ \\
\hline Yb & 3.4 & 3.8 & 4.5 & 2.3 & 2.7 & 2.4 & 2.7 & 3.2 & 3.7 & 3.7 & 2.4 & 4.6 & 4.4 & 3.2 \\
\hline & & & & & & & & $<120$ & & & $79(34)$ & & $76(31)$ & \\
\hline
\end{tabular}




\section{APPENDIX C (cont)}

USW G-1

\begin{tabular}{|c|c|c|c|c|c|c|c|c|c|c|c|c|c|}
\hline $\begin{array}{l}\text { Oepth } \\
\text { (fi) }\end{array}$ & 2601 & 2641.5 & 2869 & 2966 & 3001 & 3053 & 3137 & 3197 & 3258 & 3321 & 3372 & 3433 & 3501 \\
\hline Unit & $T C b$ & $T c t$ & Ict & Tct & Tct & Ict & Tct & Tct & Ict & Ict & ICL & ict & Tct \\
\hline $\begin{array}{l}\text { Sample } \\
\text { Lithology }\end{array}$ & 4 & 6 & 2 & 2 & 2 & 6 & 5 & 5 & 5 & 5 & 5 & 5 & 5 \\
\hline A) & 65000 & 73500 & 69900 & 75000 & 69000 & 69200 & 81500 & 69200 & 71700 & 70500 & 77300 & 81800 & 73000 \\
\hline $\mathrm{Au}$ & $<0.12$ & $<0.36$ & $<0.12$ & $<0.12$ & $<0.12$ & 0.12 & $<0.12$ & $<0.12$ & $<0.12$ & $<0.12$ & $<0.36$ & $<0.36$ & $<0.12$ \\
\hline$B a$ & 4.0 & 1050 & 570 & 810 & 570 & 1050 & 770 & $8 B O$ & 900 & 830 & 770 & 900 & 750 \\
\hline $\mathrm{Ca}$ & 8200 & 8400 & 5200 & $8 B 00$ & 6300 & 11700 & 14600 & 9400 & 15000 & 6700 & 12200 & 14900 & 9200 \\
\hline $\mathrm{Ce}$ & 87 & 255 & 103 & 140 & 117 & 147 & 105 & 96 & 101 & 90 & 101 & 99 & 105 \\
\hline $\mathrm{Cl}$ & $<300$ & $<220$ & $<260$ & $<230$ & $<250$ & $<250$ & $<220$ & $<190$ & $<230$ & $<180$ & 130 & $<190$ & $<210$ \\
\hline Co & +2.0 & $1.7(26)$ & 1.9 & 2.5 & 1.67 & 3.3 & 7.4 & 4.8 & 4.0 & 3.9 & 6.6 & 6.4 & 7.2 \\
\hline$C r$ & $<12$ & $<21$ & $<12$ & $<15$ & 3.0 & $<15$ & 27 & 9 & 4.9 & 415 & 11 & 23 & 15 \\
\hline Cs & 9.2 & $2.9(18)$ & 6.4 & 3.1 & 1.86 & 8.6 & 7.0 & 4.9 & 4.0 & 7.3 & $11.4(13)$ & 10.4 & 8.0 \\
\hline Dy & 3.3 & 5.6 & 3.0 & 5.4 & 3.9 & 3.6 & 5.9 & 3.3 & 3.9 & 3.5 & 3.3 & 4.6 & 3.9 \\
\hline Eu & & $1.01(12)$ & & & 0.59 & & & & 1.11 & & $1.33(12)$ & & 1.18 \\
\hline $\mathrm{Fe}$ & 10200 & 14000 & 9700 & 12300 & 9100 & 15700 & 24900 & 20300 & $1 d 100$ & 14700 & 25000 & 29300 & 25100 \\
\hline HF & 5.4 & 8.7 & 5.4 & 5.4 & 5.9 & 5.4 & 5.4 & 5.4 & 6.0 & 4.3 & 6.2 & 6.4 & 5.8 \\
\hline$k$ & 15400 & 48500 & 34900 & 42100 & 39700 & 28800 & 27800 & 21200 & 27100 & 30900 & 29400 & 25700 & 28100 \\
\hline La & 46 & 143 & 61 & 76 & 64 & 74 & 56 & 55 & 55 & 40 & 66 & 52 & 58 \\
\hline Lu & 0.38 & 0.38 & 0.38 & 0.51 & 0.38 & 0.38 & 0.51 & 0.25 & 0.38 & 0.38 & $0.3 \mathrm{~B}$ & 0.51 & 0.38 \\
\hline $\mathrm{Mg}$ & $<7500$ & $<6100$ & $<6700$ & $<5800$ & 1200 & $<6800$ & 4500 & 4300 & 3400 & 4200 & 4900 & 6600 & 5800 \\
\hline$M n$ & $2 ? 8$ & 360 & 940 & 410 & 500 & 580 & 490 & 253 & 430 & 193 & 510 & 284 & 500 \\
\hline $\mathrm{Na}$ & 34100 & 18600 & 24200 & 23000 & 21600 & 25300 & 24000 & 22300 & 21700 & 19300 & 22000 & 20300 & 16400 \\
\hline$R b$ & 145 & 163 & 145 & 170 & 99 & 101 & 122 & 125 & 118 & 133 & 111 & 107 & 130 \\
\hline So & $<1.5$ & $<4.3$ & $<1.5$ & $<4.3$ & $<1.5$ & $<4.3$ & $<4.3$ & $<4.3$ & $<4.3$ & $<4.3$ & $<4.3$ & $<4.3$ & $<4.3$ \\
\hline Sc & 1.1 & 2.57 & 2.2 & 3.2 & 2.43 & 2.7 & 6.3 & 4.7 & 4.31 & 3.8 & 6.72 & 9.3 & 6.94 \\
\hline Sni & 6.2 & 12.8 & 6.1 & 8.8 & 5.6 & B.5 & 10.0 & 6.4 & 5.6 & 5.7 & 6.7 & 7.6 & 5.4 \\
\hline$S r$ & $<700$ & $<70$ & $<820$ & $<610$ & 130 & $<810$ & 450 & 270 & 320 & $<520$ & $<640$ & 460 & 250 \\
\hline Ta & 1.1 & $<2.6$ & 41.7 & $<1.7$ & 1.1 & $<5$ & $<5$ & $<1.7$ & $<1.4$ & 1.1 & $<2.9$ & $<5$ & $<2.8$ \\
\hline To & $<2.3$ & $0.65(53)$ & $<2.3$ & $<? .3$ & $<1.6$ & $<2.3$ & $<2.3$ & $<2.3$ & $0.77(47)$ & $<2.3$ & $<1.9$ & $<2.3$ & $<1.9$ \\
\hline Th & 22.3 & 25.4 & 20.0 & 25.1 & 23.7 & 20.0 & 17.4 & 18.5 & 18.9 & 20.1 & 19.5 & 17.5 & 17.2 \\
\hline TI & $<1700$ & 1700 & $<2000$ & 1600 & 1140 & 1900 & 3200 & 2160 & 2120 & 1440 & 2660 & 3700 & 2800 \\
\hline$U$ & 3.22 & 4.22 & 4.01 & 4.25 & 4.14 & 3.76 & 2.52 & 1.51 & 3.38 & 3.12 & 3.17 & 3.02 & 3.16 \\
\hline$v$ & $<21$ & 13 & $<22$ & 16 & $<19$ & 22 & 57 & 33 & 37 & 22 & 52 & 71 & 61 \\
\hline Yb & 2.2 & 3.8 & 2.4 & 2.8 & 3.0 & 2.5 & 3.6 & 2.5 & 2.7 & 2.7 & 2.7 & 2.5 & 2.5 \\
\hline $2 n$ & & $46(46)$ & & & $<110$ & & & & $47(34)$ & & $92(28)$ & & $62(36)$ \\
\hline
\end{tabular}




\section{APPENDIX C (cont)}

\begin{tabular}{|c|c|c|c|c|c|c|c|c|c|c|c|c|c|}
\hline \multirow[b]{2}{*}{$\begin{array}{l}\text { Denth } \\
(\mathrm{ft})\end{array}$} & \multicolumn{13}{|c|}{ USH G-I } \\
\hline & 3549 & 3598 & 3659 & 3706 & 3755 & 3810 & 3850 & 3914 & 3954 & 3997 & 4052 & 4095 & 4208 \\
\hline Untt & ict & Tfb & T fb & $T f D$ & $T \mathrm{fb}$ & ifo & $I f b$ & T fo & TIr & $\pi r$ & Tir & IIr & $I 1 r$ \\
\hline $\begin{array}{l}\text { Sample } \\
\text { LItnol agy }\end{array}$ & 4 & 7 & 8 & 8 & $B$ & 8 & B & 8 & 5 & 6 & 6 & 6 & 6 \\
\hline A) & 79300 & 78500 & $84100(5)$ & 96500 & 81300 & 95500 & 844J0 & 101000 & 80600 & 78800 & 76400 & 76200 & 72600 \\
\hline $\mathrm{Au}$ & 40.36 & $<0.36$ & $0.0030(90)$ & $<0.36$ & $<0.36$ & $<0.36$ & $c 0.36$ & $<0.36$ & $<0.12$ & $<0.36$ & $<0.12$ & $<0.12$ & $<0.12$ \\
\hline Ba & 390 & 1380 & $1380(5)$ & 1160 & 2700 & 910 & 1330 & 1680 & 1760 & 1370 & 1040 & 970 & 960 \\
\hline $\mathrm{Ca}$ & 3500 & 21400 & $21900(8)$ & 25500 & 25200 & 32200 & 29600 & 31800 & 9800 & 9900 & 12300 & 7400 & 9200 \\
\hline Ce & 110 & 130 & $129(5)$ & 147 & 137 & 156 & 146 & 162 & 161 & 154 & 125 & 125 & 110 \\
\hline $\mathrm{Cl}$ & $<260$ & $<210$ & $<200$ & $<190$ & $<270$ & $<230$ & $<280$ & $<270$ & $<240$ & $\varangle 260$ & $<310$ & $<250$ & $<260$ \\
\hline Co & $0.76(47)$ & 3.2 & $4.1(6)$ & 5.9 & 4.8 & $7.1(10)$ & 6.0 & 7.2 & 2.4 & 2.7 & 2.4 & 3.0 & $3.9(11)$ \\
\hline$C r$ & $<21$ & $<19$ & $2.6(45)$ & $<19$ & $<19$ & $5.4(63)$ & $<22$ & $<22$ & $<19$ & $<19$ & $<16$ & $<13$ & $4.8(33)$ \\
\hline Cs & $48(13)$ & 3.9 & $2.9(9)$ & 3.4 & 3.9 & $4.2(10)$ & 7.4 & 2.3 & 10.6 & 10.4 & 5.3 & 6.5 & $3.04(11)$ \\
\hline Dy & 5.8 & 4.9 & $5.2(15)$ & 5.6 & 5.5 & 6.3 & 4.1 & 5.8 & 5.4 & 5.2 & 4.8 & 5.5 & 4.5 \\
\hline $\mathrm{Eu}$ & $0.61(15)$ & & $2.06(5)$ & & & $2.84(10)$ & & & & & & & $1.20(11)$ \\
\hline $\mathrm{Fe}$ & 11100 & 20300 & $21000(5)$ & 27600 & 27200 & $47800(6)$ & 28200 & 39800 & 14600 & 18700 & 14700 & 13100 & $16500(8)$ \\
\hline Hf & 6.4 & 8.6 & $7.5(5)$ & 9.7 & 7.5 & 10.2 & 8.6 & 10.7 & 8.6 & 7.5 & 6.4 & 6.4 & 6.8 \\
\hline$k$ & 22500 & 24700 & $33600(15)$ & 27600 & 27400 & 3900 & 11900 & 9700 & 41100 & 28700 & 25100 & 28400 & 30200 \\
\hline La & 52 & 73 & $65(7)$ & 79 & 73 & 80 & 74 & 85 & 94 & 19 & 71 & 64 & 63 \\
\hline Lu & 0.51 & 0.49 & $0.41(6)$ & 0.37 & 0.48 & 0.37 & 0.48 & 0.48 & 0.48 & 0.60 & 0.37 & 0.37 & 0.37 \\
\hline $\mathrm{Mg}$ & $<7500$ & $<5500$ & $2700\langle 45\rangle$ & 9300 & 4600 & 15400 & 6000 & 9300 & 5300 & $<7100$ & $<7600$ & 3500 & 3300 \\
\hline Mn & 122 & 310 & $515(6)$ & 193 & 660 & 340 & 1040 & 720 & 270 & 410 & 650 & 400 & 400 \\
\hline $\mathrm{Na}$ & 31800 & 25100 & $26300(4)$ & 21900 & 25200 & 24700 & 26400 & 29400 & 18400 & 32400 & 30300 & 32500 & 25600 \\
\hline$R D$ & 118 & 129 & $114(6)$ & 170 & 79 & $<92$ & 65 & 47 & 177 & 103 & 118 & 119 & 136 \\
\hline Sb & $<4.3$ & $<4.3$ & $0.25(51)$ & $<4.3$ & $<4.3$ & $<4.3$ & $<4.3$ & $<4.3$ & 4.3 & 44.3 & $<4.3$ & $<1.5$ & $<4.3$ \\
\hline Sc & 2.57 & 8.8 & $8.1(6)$ & 10.1 & 9.0 & $11.5(8)$ & 10.6 & 11.9 & 2.7 & 2.9 & 2.7 & 2.8 & $4.1(8)$ \\
\hline Sm & 7.4 & 8.1 & $10.2(7)$ & 9.1 & 8.9 & $9.1(20)$ & 9.6 & 9.7 & 7.7 & 9.1 & 4.6 & 3.4 & $4.8(21)$ \\
\hline Sr & $<710$ & $<520$ & $570(33)$ & 670 & 560 & 870 & 750 & 800 & 410 & $<740$ & $<810$ & $<620$ & 230 \\
\hline $\mathrm{Td}$ & $<3.4$ & $<5$ & $0.81(13)$ & $<5$ & $<5$ & $=3.0$ & 5 & $<5$ & $<5$ & $<5$ & $<5$ & $<1.7$ & $2.9(45)$ \\
\hline TO & $<1.9$ & $<2.3$ & $1.05(11)$ & $<2.3$ & $<2.3$ & 41.7 & $<2.3$ & $<2.3$ & $<2.3$ & $<2.3$ & $<2.3$ & 2.3 & $<1.8$ \\
\hline Th & 28.5 & 14.9 & $15.3(4)$ & 18.6 & 15.3 & 17.8 & 15.5 & 19.6 & 18.5 & 17.3 & 19.0 & 18.9 & 17.6 \\
\hline Ti & $<1800$ & 4300 & $4400(11)$ & 5500 & 4100 & 5500 & 4500 & 5500 & 2310 & 2220 & 1420 & 1400 & 2070 \\
\hline$u$ & 4.07 & 2.66 & $2.88(6)$ & 4.51 & 2.82 & 3.43 & 4.08 & 3.69 & 4.21 & 3.27 & 2.97 & 4.71 & 2.85 \\
\hline$v$ & $<19$ & 37 & $40(12)$ & 52 & 43 & 53 & 47 & 60 & 16 & 30 & 22 & 22 & 39 \\
\hline YD & 3.7 & 3.3 & $2.70(5)$ & 2.2 & 4.1 & $3.1(32)$ & 4.1 & 4.5 & 3.9 & 3.4 & 2.9 & 3.2 & $2.4(33)$ \\
\hline $2 n$ & $<92$ & & $114(14)$ & & & $116(25)$ & & & & & & & $89(27)$ \\
\hline $\mathrm{Zr}$ & & & $275(19)$ & & & & & & & & & & \\
\hline
\end{tabular}


USW 6-1

\begin{tabular}{|c|c|c|c|c|c|c|c|c|c|c|c|}
\hline \multirow[b]{2}{*}{$\begin{array}{l}\text { Oepth } \\
(\mathrm{ft})\end{array}$} & \\
\hline & 4296 & 4401 & 4451 & 4504 & 4612 & 4652 & 4700 & 4750 & 4805 & 4848 & 4913 \\
\hline Unt t & $\mathrm{Il} r$ & Tlr & Tlr & $\operatorname{Tlr}$ & $T / r$ & $T I r$ & $\operatorname{Tlr}$ & $\pi r$ & $\operatorname{IIr}$ & $\operatorname{lr}$ & $\mathrm{Tlr}$ \\
\hline $\begin{array}{l}\text { Samole } \\
\text { Lithology }\end{array}$ & 6 & 5 & 6 & 6 & 6 & 6 & 6 & 6 & 6 & 6 & 6 \\
\hline A) & 14300 & 77500 & 68700 & 70600 & 73000 & 73400 & 71300 & 70500 & 73300 & 72100 & 67900 \\
\hline Au & $<0.12$ & $<0.12$ & $<0.12$ & $<0.12$ & 0.12 & $<0.12$ & $<0.12$ & $<0.12$ & $<0.12$ & $<0.12$ & $<0.12$ \\
\hline $\mathrm{Ba}$ & 780 & 770 & 850 & 690 & B10 & 750 & 760 & 850 & 930 & 840 & 720 \\
\hline $\mathrm{Ca}$ & 7400 & 9700 & 8800 & 7600 & 7100 & 7000 & 6000 & 6500 & 9700 & 7100 & 7600 \\
\hline $\mathrm{Ce}$ & 110 & 99 & 98 & 88 & 106 & 109 & 107 & 101 & 126 & 124 & 92 \\
\hline $\mathrm{Cl}$ & $<240$ & $<220$ & $<230$ & $<240$ & $<220$ & $<210$ & $<190$ & $<230$ & $<220$ & $<200$ & $<180$ \\
\hline Co & 2.3 & 2.6 & 1.5 & 2.3 & 3.3 & 2.8 & $2.7(13)$ & 2.9 & 2.3 & 2.9 & 2.1 \\
\hline $\mathrm{Cr}$ & $<16$ & $<13$ & $<13$ & $<13$ & $<16$ & $<16$ & $4.2(38)$ & 13 & $<16$ & $<16$ & $<13$ \\
\hline Cs & 4.6 & 3.2 & 5.7 & 4.7 & 4.7 & 4.3 & $3.67(10)$ & 4.1 & 4.1 & 4.6 & 3.8 \\
\hline $0 y$ & 3.8 & 3.5 & 3.8 & 3.8 & 3.9 & 3.6 & 3.7 & 3.7 & 4.3 & 4.6 & 3.9 \\
\hline Eu & & & & & & & $0.81(11)$ & & & & \\
\hline $\mathrm{Fe}$ & 12900 & 12100 & 9600 & 12000 & 15000 & 13500 & $12100(8)$ & 14000 & 12100 & 13200 & 11200 \\
\hline Hf & 5.4 & 6.4 & 5.4 & 5.4 & 5.4 & 5.4 & 6.0 & 6.4 & 5.4 & 6.4 & 5.4 \\
\hline$k$ & 33800 & 34100 & 26500 & 31900 & 31700 & 32300 & 35600 & 33600 & 34000 & 35300 & 38100 \\
\hline La & 54 & 57 & 54 & 54 & 54 & 63 & 56 & 56 & 63 & 73 & 53 \\
\hline Lu & 0.49 & 0.37 & 0.37 & 0.38 & 0.37 & 0.37 & 0.37 & 0.37 & 0.37 & 0.37 & 0.48 \\
\hline $\mathrm{Mg}$ & 5100 & 3900 & $<5900$ & 3700 & 5300 & $<5400$ & 3300 & 3600 & 4300 & 3600 & 4000 \\
\hline$M_{n}$ & 520 & 380 & 320 & 350 & 340 & 390 & 300 & 330 & 410 & 320 & 380 \\
\hline $\mathrm{Na}$ & 26700 & 26000 & 31100 & 24400 & 24300 & 22000 & 21400 & 21600 & 21400 & 21800 & 14200 \\
\hline $\mathrm{Rb}$ & 129 & 125 & 117 & 113 & 115 & 155 & 171 & 146 & 135 & 145 & 168 \\
\hline Sb & $<4.3$ & $<1.5$ & $<1.5$ & $<1.5$ & $<4.3$ & $<4.3$ & $<1.5$ & $<1.5$ & $<4.3$ & $<4.3$ & $<1.5$ \\
\hline Sc & 2.7 & 2.7 & 2.0 & 2.7 & 3.0 & 3.1 & $2.75(9)$ & 2.7 & 2.3 & 3.0 & 2.0 \\
\hline $5 m$ & 4.0 & 3.8 & 3.1 & 3.4 & 4.5 & 4.1 & $4.5(22)$ & 4.0 & 5.3 & 4.4 & 3.0 \\
\hline$S r$ & $<690$ & $<620$ & $<580$ & $<610$ & 300 & $<600$ & 220 & $<600$ & $<670$ & $<560$ & $<540$ \\
\hline Ta & $<1.7$ & $<1.7$ & $<1.7$ & $1.1(100)$ & $<1.7$ & 2.2 & $1.1(100)$ & $<1.7$ & $<1.7$ & $1.1(100)$ & $<1.7$ \\
\hline To & $<2.3$ & $<2.3$ & $<2.3$ & $<2.2$ & $<2.3$ & $<2.3$ & $<1.7$ & $<2.3$ & $<2.3$ & $<2.3$ & $<2.3$ \\
\hline Th & 19.4 & 17.8 & 17.4 & 17.6 & 16.7 & 20.0 & 18.7 & 18.1 & 19.9 & 21.5 & 19.9 \\
\hline $\mathrm{Ti}$ & 1220 & 1590 & 1130 & 1710 & 1840 & 1360 & 1560 & 1510 & 1340 & 1210 & 1540 \\
\hline$U$ & 2.61 & 2.95 & 3.07 & 1.69 & 2.57 & 2.80 & 3.06 & 2.89 & 2.67 & 2.99 & 2.93 \\
\hline$v$ & 16 & 21 & 16 & 19 & 29 & 17 & 20 & 17 & 18 & 18 & 13 \\
\hline Yo & 2.7 & 2.7 & 2.1 & 2.9 & 2.6 & 3.1 & $2.2(36)$ & 2.3 & 3.8 & 2.8 & 3.6 \\
\hline $2 \pi$ & & & & & & & $49(43)$ & & & & \\
\hline $\mathrm{Zr}$ & & & & & & & $260(62)$ & & & & \\
\hline
\end{tabular}


APPENDIX $C$ (cont)

USW G-1

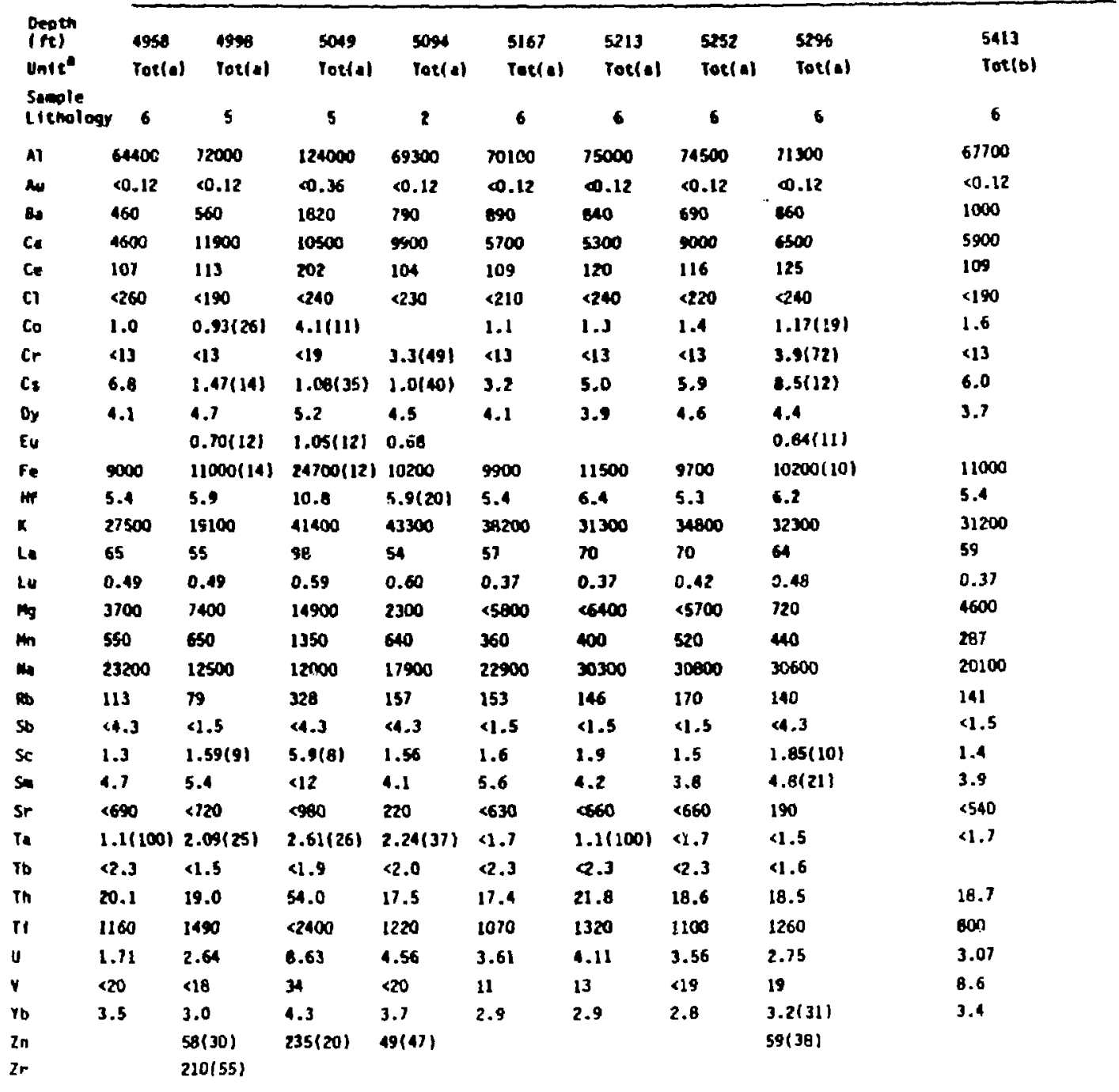

- Letters in parentheses indicate subunits with the sequence of older tuffs and lavas tdentifled in drill hole uSW G-1 by Soengler et 11 . (1901). Thus far, these subunits have not been correlated to equivalent units in other Yucca Hountain orfll holes. 


\begin{tabular}{|c|c|c|c|c|c|c|c|c|c|c|c|c|}
\hline \multirow[b]{2}{*}{$\begin{array}{l}\text { Depth } \\
(\mathrm{ft})\end{array}$} & \multicolumn{12}{|c|}{ USA G-1 } \\
\hline & $\begin{array}{l}5458 \\
\operatorname{Tot}(c)\end{array}$ & $\begin{array}{l}5499 \\
\operatorname{Tot}(c)\end{array}$ & $\begin{array}{l}5560 \\
\operatorname{Tot}(c)\end{array}$ & $\begin{array}{l}5596 \\
\operatorname{Tot}(c)\end{array}$ & $\begin{array}{l}5637 \\
\text { Tot(c) }\end{array}$ & $\begin{array}{l}5699 \\
\operatorname{Iot}(c)\end{array}$ & $\begin{array}{l}5747 \\
\text { Tot(c) }\end{array}$ & $\begin{array}{l}5803 \\
\operatorname{Tot}(c)\end{array}$ & $\begin{array}{l}5848 \\
\operatorname{Tot}(c)\end{array}$ & $\begin{array}{l}5898 \\
\operatorname{Tot}(c)\end{array}$ & $\begin{array}{l}5980 \\
\operatorname{Tot}(c)\end{array}$ & $\begin{array}{l}5980 \\
\text { Tot(c) }\end{array}$ \\
\hline $\begin{array}{l}\text { Sample } \\
\text { Li thology }\end{array}$ & 5 & 6 & 5 & 6 & 6 & 6 & 6 & 2 & 2 & 2 & 6 & 5 \\
\hline AI & 83500 & 72800 & 81200 & 82700 & 17900 & 88500 & 85000 & 85900 & 84800 & 112000 & 85900 & 88400 \\
\hline Au & $<0.12$ & $<0.12$ & $<0.12$ & $<0.12$ & $<0.12$ & $<0.12$ & $<0.12$ & $<0.12$ & $<0.12$ & $<0.36$ & $<0.12$ & $<.12$ \\
\hline Ba & 1960 & 1210 & 3120 & 1740 & 1450 & 1570 & 1710 & 1570 & 1950 & 1130 & 1530 & 1800 \\
\hline Ca & 14400 & 12100 & 21000 & 18500 & 15200 & 21200 & 17000 & 27500 & 14600 & 20000 & 19100 & 22100 \\
\hline Ce & 125 & 115 & 118 & i01 & 119 & 117 & 134 & 119 & 121 & 148 & 113 & 121 \\
\hline Cl & $<200$ & $<260$ & $<230$ & $\$ 260$ & 250 & $<740$ & $<240$ & $<300$ & $<270$ & $<250$ & $<270$ & $<300$ \\
\hline co & $2.35(12)$ & 2.3 & 4.0 & 3.7 & $3.6(11)$ & 6.0 & 5.8 & $4.4(10)$ & 6.8 & 5.2 & $8.2(10)$ & $7.3(10)$ \\
\hline Cr & $<16$ & $<13$ & $<16$ & $<13$ & $5.5(40)$ & $<16$ & $<13$ & $<16$ & $<16$ & $<22$ & $<16$ & 8 \\
\hline Cs & $1.9(9)$ & 9.7 & 2.2 & 6.4 & 9.2 & 3.8 & 6.0 & $1.24(28)$ & 1.8 & 9.9 & $10.4(10)$ & $1.48(30)$ \\
\hline Dy & 6.0 & 2.6 & 3.2 & 1.9 & 3.1 & 2.1 & 3.7 & 3.5 & 3.0 & 4.2 & 4.1 & 4.2 \\
\hline Eu & $1.21(12)$ & & & & 1.28 & & & $1.41(12)$ & & & $1.40(12)$ & $1.75(12)$ \\
\hline Fe & $19200(12)$ & 14100 & 19600 & 18600 & 18200 & 23200 & 23200 & $20300(12)$ & 24200 & 23900 & $24800(13)$ & $28400(12)$ \\
\hline$H$ & 11.9 & 6.4 & 6.4 & 6.4 & B.o & 7.5 & 7.5 & & 7.5 & 8.6 & 7.7 & \\
\hline$x$ & 20900 & 25700 & 22400 & 23500 & 25200 & 24900 & 25400 & 17900 & 22200 & 31400 & 17700 & 23400 \\
\hline t. & 75 & 74 & 60 & 63 & 69 & 78 & 60 & 83 & 65 & 96 & 66 & 71 \\
\hline tu & 0.48 & 0.25 & 0.38 & 0.25 & 0.33 & 0.25 & 0.37 & 0.25 & 0.37 & 0.38 & 0.38 & 0.37 \\
\hline m & 11300 & 5400 & 6100 & 5900 & 5200 & 5700 & 7100 & 2900 & 9300 & 9300 & 7800 & 10300 \\
\hline Mn & 510 & 420 & 690 & 630 & 517 & 640 & 550 & 630 & 550 & 590 & 780 & 560 \\
\hline Ma & 18900 & 27000 & 20800 & 31600 & 33700 & 29800 & 28600 & 32300 & 33000 & 19500 & 33100 & 31200 \\
\hline Ro & 145 & 120 & 118 & 124 & 125 & 133 & 145 & 113 & 134 & 318 & 128 & 136 \\
\hline so & $<4.3$ & $<4.3$ & $<4.3$ & $<4.3$ & $<1.5$ & 4.3 & $<4.3$ & $<4.3$ & $<4.3$ & $<4.3$ & $<4.3$ & $<4.3$ \\
\hline Sc & $3.06(8)$ & 2.3 & 3.0 & 2.7 & 3.0 & 4.4 & 4.5 & $3.64(8)$ & 4.2 & 4.3 & $5.8(8)$ & $6.6(8)$ \\
\hline son & 6.6 & 4.0 & 5.0 & 3.9 & 4.5 & 4.9 & 4.9 & 4.7 & 5.5 & 5.7 & 5.4 & 5.9 \\
\hline Sr & 650 & 40 & 1420 & $<750$ & 360 & 860 & 600 & 630 & 560 & 580 & 610 & 710 \\
\hline Ta & $1.9(38)$ & $<1.7$ & $<1.7$ & $<1.7$ & $<1.5$ & $<5$ & $<1.7$ & $<1.5$ & $<1.7$ & $<5$ & $1.1(100)$ & $<2.8$ \\
\hline Tb & $<1.6$ & $<2.3$ & $<2.3$ & $<2.3$ & $<1.5$ & $<2.3$ & $<2.3$ & $<1.8$ & $<2.3$ & $<2.3$ & $<1.8$ & $<1.8$ \\
\hline Th & 21.0 & 17.4 & 17.7 & 13.4 & 17.2 & 14.7 & 17.1 & 14.7 & 13.9 & 24.0 & 15.7 & 14.5 \\
\hline TH & 2020 & 1620 & 2860 & 1280 & 2200 & 2290 & 2440 & 2950 & 2960 & 3090 & 2640 & 3140 \\
\hline$u$ & 3.24 & 2.27 & 2.16 & 2.11 & 2.88 & 2.39 & 2.56 & 2.52 & 3.06 & 1.57 & 2.10 & 2.50 \\
\hline$v$ & 18 & 19 & 33 & 30 & 33 & 45 & 40 & 39 & 47 & 53 & 60 & 56 \\
\hline Yo & 3.2 & 2.3 & 2.9 & 2.4 & 2.3 & 2.4 & 2.3 & 2.2 & 2.4 & 3.9 & 3.0 & 2.3 \\
\hline $2 n$ & $99(26)$ & & & & $63(69)$ & & & $57(35)$ & & & $<35$ & \\
\hline
\end{tabular}

- Letters in parentheses indicate subuntes the sequence of older tuffs and lavas Identified in drill mole USW G-1 by Spengler Letters in parentheses indicate subunits with the sequence of older cuffs and lavas idenciried hucca Mountain drill holes. 
APPENDIX C (cont)

\begin{tabular}{|c|c|c|c|c|c|c|c|c|}
\hline \multirow[b]{2}{*}{ Depth } & \multicolumn{3}{|c|}{ USH G-2 } & \multicolumn{2}{|c|}{ USW EU-3 } & \multicolumn{3}{|c|}{$\begin{array}{l}\text { Tulf of Caifeo } \\
\text { Prow Pass. northern vuces Hountion }\end{array}$} \\
\hline & 547 & 675 & 1691 & 1322 & 1498 & $3-15-82-5$ & $3-15-82-9$ & RUG2a-1 \\
\hline Unte & $T p D$ & TDD & TDt & Int & The & Tht & Tht & Int \\
\hline $\begin{array}{l}\text { Samole } \\
\text { Lithology }\end{array}$ & 1 & 5 & 6 & 1 & 1 & 6 & 6 & 9 \\
\hline AI & 75000 & 85000 & 62000 & 68000 & 66000 & 65000 & 61000 & 63000 \\
\hline Au & $<0.02$ & $<0.02$ & $0.06(15)$ & $<0.02$ & $<0.02$ & $<0.01$ & $<0.01$ & $<0.02$ \\
\hline Ba & 1750 & 2150 & $<140$ & $<200$ & 235 & 330 & 160 & 355 \\
\hline ca & 9000 & 16000 & 16000 & 3200 & 6000 & 8000 & 4600 & 5200 \\
\hline Ce & 161 & 185 & 35 & 76 & 106 & 53 & 73 & 81 \\
\hline Cl & 260 & $<110$ & $<90$ & 580 & 840 & $<70$ & $<60$ & 330 \\
\hline Co & & & & & & & & \\
\hline Cr & $<2$ & 4 & $<2$ & $<2$ & 6 & $<2$ & 4.3 & $<2$ \\
\hline cs & 4.5 & 6.8 & 11.1 & 6.2 & 3.7 & 6.6 & 3.8 & 5.5 \\
\hline Dy & 6.2 & 8.3 & 3.5 & 5.2 & 7.5 & 4.2 & 3.6 & 4.7 \\
\hline Eu & 1.50 & 1.92 & 0.24 & 0.24 & 0.24 & 0.25 & 0.27 & 0.33 \\
\hline Fe & 10400 & 12100 & 6100 & 6100 & 8500 & 5700 & 7500 & 6500 \\
\hline Hf & 8.3 & 9.1 & 4.2 & 4.6 & 6.2 & 3.6 & 4.1 & 3.2 \\
\hline $\boldsymbol{x}$ & 34020 & 21500 & 29500 & 44000 & 40500 & 55000 & 66500 & 45000 \\
\hline La & 87 & 116 & 23.9 & 43 & 68 & 30.3 & 37 & 44 \\
\hline Lu & 0.50 & 0.49 & 0.17 & 0.5 & 0.8 & 0.30 & 0.24 & 0.67 \\
\hline $\mathbf{H g}$ & 8000 & 9000 & $<2000$ & $<3000$ & 3000 & $<1000$ & 2000 & $<2000$ \\
\hline Mn & 620 & 680 & 141 & 520 & 560 & 290 & 260 & 380 \\
\hline Ma & 26150 & 10000 & 8300 & 27000 & 25350 & 4600 & 3805 & 17450 \\
\hline$R D$ & 141 & 87 & 161 & 188 & 160 & 220 & 196 & 206 \\
\hline so & $<0.8$ & $<1.0$ & & & $<0.5$ & $<0.4$ & $<0.4$ & $<0.6$ \\
\hline se & 4.2 & 4.8 & 2.3 & 2.3 & 1.8 & 2.5 & 2.8 & 2.5 \\
\hline$s m$ & 10.1 & 12.2 & 4.6 & $<7$ & 9.5 & 5.0 & 5.2 & 5.2 \\
\hline sr & 290 & 1600 & $<100$ & $<300$ & $<300$ & $<200$ & $<200$ & $<200$ \\
\hline TA & & & & & & & & \\
\hline To & 0.5 & 0.7 & 0.29 & 0.5 & 0.7 & 0.19 & 0.34 & 0.5 \\
\hline Th & 22.3 & 26 & 24 & 24.3 & 21.3 & 23.1 & 20.9 & 26.0 \\
\hline TI & 2100 & 2100 & $<600$ & $<700$ & 1000 & $<500$ & 800 & $<500$ \\
\hline v & 3.2 & 1.54 & 2.44 & 4.6 & 4.2 & 3.02 & 2.61 & 4.6 \\
\hline v & 12 & 21 & $<6$ & $\Leftrightarrow$ & $<8$ & $<5$ & $<6$ & $<6$ \\
\hline Yo & 3.8 & 4.4 & $<2.4$ & 2.9 & 3.4 & 2.3 & 1.8 & 2.3 \\
\hline $2 n$ & & & & & & & & \\
\hline $2 r$ & 430 & 380 & $<300$ & 260 & $<300$ & $<100$ & $<100$ & $\ll 100$ \\
\hline
\end{tabular}

Awerada State Coordinates (ft) for sample locations: 3-15-62-5-786500H, 551000E: 3-15-82-9×786700N. 551400E, RHG2a-1 =787400N, $553250 E$. 


\section{ANALYTICAL VALUES FOR NBS STANDARDS \\ BY AUTOMATED NEUTRON ACTIVATION ANALYSIS}

Values are from Tables 1 and 2 of Garcia et al. (1982). Analyses were under conditions identical to those for values of Appendix C. Values provided by the NBS in parentheses are uncertified. Standard deviations are based on 24 analyses of SRM 1632a and 50 analyses of SRM 1633a.

\begin{tabular}{|c|c|c|c|c|c|c|}
\hline \multirow[b]{2}{*}{ Element } & \multicolumn{3}{|c|}{ SRM 1632a Coal } & \multicolumn{3}{|c|}{ SRM 1633a Coal Fly Ash } \\
\hline & $\begin{array}{c}\text { Average } \\
(\mathrm{ppm})\end{array}$ & $\begin{array}{l} \pm 2 \sigma \\
(\%)\end{array}$ & $\begin{array}{c}\text { NBS } \\
(p p m)\end{array}$ & $\begin{array}{c}\text { Average } \\
(\mathrm{ppm})\end{array}$ & $\begin{array}{l} \pm 2 \sigma \\
(\%)\end{array}$ & $\begin{array}{c}\text { NBS } \\
(\mathrm{ppm})\end{array}$ \\
\hline Al & 30700 & 8.2 & $(30700)$ & 142000 & 4.4 & $(140000)$ \\
\hline $\mathrm{Ba}$ & 150 & 34 & & 1500 & 12 & $(1500)$ \\
\hline $\mathrm{Ca}$ & 2700 & 13 & & 11200 & 14 & 11100 \\
\hline $\begin{array}{l}\text { Ce } \\
\mathrm{Cl}\end{array}$ & $\begin{array}{r}31.1 \\
897\end{array}$ & $\begin{array}{c}22 \\
5.2\end{array}$ & $(30)$ & 183 & 22 & $(180)$ \\
\hline Co & 6.6 & 34 & $(6.8)$ & 46.2 & 7.6 & $(46)$ \\
\hline $\mathrm{Cr}$ & 36 & 34 & 34 & 197 & 13 & 196 \\
\hline Cs & 2.4 & 70 & $(2.4)$ & 10.6 & 21 & $(11)$ \\
\hline Dy & 2.56 & 20 & & & & \\
\hline Eu & 0.51 & 32 & $(0.54)$ & 2.98 & 22 & \\
\hline $\mathrm{Fe}$ & 11200 & 16 & 11100 & 95000 & 6.4 & 94000 \\
\hline $\mathrm{HF}$ & 1.8 & 36 & $(1.6)$ & 7.78 & 22 & $(7.6)$ \\
\hline K & 4300 & 30 & & 18400 & 15 & 18800 \\
\hline La & 15 & 34 & & 100 & 46 & \\
\hline Lu & 0.18 & 74 & & 0.93 & 21 & \\
\hline $\mathrm{Mg}$ & 1400 & 32 & & 4500 & 22 & 4550 \\
\hline Mn & 33.7 & 7.2 & 28 & 191 & 4.5 & $(190)$ \\
\hline $\mathrm{Na}$ & 884 & 7.2 & & 1720 & 5.6 & 1700 \\
\hline $\mathrm{Rb}$ & & . & & 130 & 40 & 131 \\
\hline $\mathrm{Sb}$ & & & & 7.8 & 40 & $(7)$ \\
\hline Sc & 6.56 & 7.0 & $(6.3)$ & 40.6 & 6.3 & (40) \\
\hline Sm & 2.5 & 28 & & 20 & 44 & \\
\hline$S r$ & & & & 819 & 13 & 830 \\
\hline $\mathrm{Ta}$ & & & & 2.0 & 46 & \\
\hline Tb & & & & 2.3 & 58 & \\
\hline Th & 4.8 & 24 & 4.5 & 24.8 & 13 & 24.7 \\
\hline $\mathrm{Ti}$ & 1720 & 20 & $(1750)$ & 8060 & 9.2 & $(8000)$ \\
\hline U & 1.28 & 12 & 1.28 & 10.2 & 4.2 & 10.2 \\
\hline V & 46.9 & 11 & 44 & 301 & 5.2 & $(300)$ \\
\hline$Y b$ & & & & 10 & 36 & \\
\hline $\mathrm{Zn}$ & & & & 220 & 46 & 220 \\
\hline & & & & & & \\
\hline
\end{tabular}


GLASS COMPOSITIONS DETERMINED BY ELECTRON MICROPROBE FOR TUFFS OF YUCCA MOUNTAIN, NEVADA

Drill Hole USW G-2

\begin{tabular}{|c|c|c|c|c|c|c|c|c|}
\hline $\begin{array}{l}\text { Depth } \\
\text { (ft) } \\
\text { Unit }\end{array}$ & 331 & 331 & 331 & 331 & 331 & 331 & 331 & 331 \\
\hline $\begin{array}{l}\mathrm{SiO} 2 \\
\mathrm{TiO} 2 \\
\mathrm{Al} 203 \\
\mathrm{Fe} 203 \\
\mathrm{MgO} \\
\mathrm{CaO} \\
\mathrm{BaO} \\
\mathrm{Na2O} \\
\mathrm{K} 20\end{array}$ & $\begin{array}{c}73.7 \\
0.14 \\
12.1 \\
0.91 \\
0.00 \\
0.28 \\
0.00 \\
4.26 \\
4.64\end{array}$ & $\begin{array}{c}72.5 \\
0.18 \\
12.3 \\
0.88 \\
0.00 \\
0.26 \\
0.00 \\
4.56 \\
4.43\end{array}$ & $\begin{array}{c}72.9 \\
0.15 \\
11.8 \\
0.89 \\
0.00 \\
0.25 \\
0.00 \\
4.45 \\
4.53\end{array}$ & $\begin{array}{c}72.4 \\
0.16 \\
12.2 \\
0.88 \\
0.00 \\
0.26 \\
0.00 \\
4.62 \\
4.34\end{array}$ & $\begin{array}{c}72.2 \\
0.13 \\
12.1 \\
0.69 \\
0.00 \\
0.26 \\
0.00 \\
4.68 \\
4.35\end{array}$ & $\begin{array}{c}73.3 \\
0.10 \\
12.1 \\
0.81 \\
0.00 \\
0.27 \\
0.00 \\
4.55 \\
4.37\end{array}$ & $\begin{array}{c}73.0 \\
0.13 \\
11.9 \\
0.84 \\
0.00 \\
0.24 \\
0.00 \\
4.59 \\
4.00\end{array}$ & $\begin{array}{c}73.1 \\
0.10 \\
11.3 \\
0.65 \\
0.00 \\
0.23 \\
0.00 \\
4.52 \\
3.92\end{array}$ \\
\hline Total & 96.0 & 95.1 & 95.0 & 94.9 & 94.4 & 95.5 & 94.8 & 93.8 \\
\hline \multicolumn{9}{|c|}{ Cation Percent } \\
\hline $\begin{array}{l}\mathrm{Si} \\
\mathrm{Ti} \\
\mathrm{Al} \\
\mathrm{Fe} \\
\mathrm{Mg} \\
\mathrm{Ca} \\
\mathrm{Ba} \\
\mathrm{Na} \\
\mathrm{K}\end{array}$ & $\begin{array}{c}71.4 \\
0.10 \\
13.8 \\
0.66 \\
0.00 \\
0.29 \\
0.00 \\
8.01 \\
5.74\end{array}$ & $\begin{array}{c}70.7 \\
0.13 \\
14.1 \\
0.65 \\
0.00 \\
0.27 \\
0.00 \\
8.62 \\
5.51\end{array}$ & $\begin{array}{c}71.3 \\
0.11 \\
13.6 \\
0.65 \\
0.00 \\
0.26 \\
0.00 \\
8.44 \\
5.65\end{array}$ & $\begin{array}{c}70.8 \\
0.12 \\
14.0 \\
0.65 \\
0.00 \\
0.27 \\
0.00 \\
8.75 \\
5.41\end{array}$ & $\begin{array}{c}70.8 \\
0.10 \\
14.0 \\
0.51 \\
0.00 \\
0.27 \\
0.00 \\
8.90 \\
5.44\end{array}$ & $\begin{array}{c}71.2 \\
0.07 \\
13.8 \\
0.59 \\
0.00 \\
0.28 \\
0.00 \\
8.57 \\
5.42\end{array}$ & $\begin{array}{c}71.5 \\
0.10 \\
13.8 \\
0.62 \\
0.00 \\
0.25 \\
0.00 \\
8.72 \\
5.00\end{array}$ & $\begin{array}{c}72.4 \\
0.07 \\
13.2 \\
0.48 \\
0.00 \\
0.24 \\
0.00 \\
8.68 \\
4.95\end{array}$ \\
\hline \multicolumn{9}{|c|}{$\mathrm{Si} /(\mathrm{Al}+\mathrm{Fe})$} \\
\hline \multicolumn{9}{|c|}{$(\mathrm{A})+\mathrm{Fe}) /(2 \mathrm{Mg}+2 \mathrm{Ca}+2 \mathrm{Ba}+\mathrm{Na}+\mathrm{K})$} \\
\hline $\begin{array}{l}\mathrm{K} \\
\mathrm{Na} \\
\mathrm{Ca}+\mathrm{Mg}\end{array}$ & $\begin{array}{r}40.9 \\
57.0 \\
2.1\end{array}$ & $\begin{array}{r}38.3 \\
59.9 \\
1.9\end{array}$ & $\begin{array}{c}\text { Mo1\% Exc } \\
39.4 \\
58.8 \\
1.8\end{array}$ & $\begin{array}{l}\text { ngeable } \\
37.5 \\
60.6 \\
1.9\end{array}$ & $\begin{array}{c}\text { Cations } \\
37.2 \\
60.9 \\
1.9\end{array}$ & $\begin{array}{r}38.0 \\
60.1 \\
2.0\end{array}$ & $\begin{array}{r}35.8 \\
62.4 \\
1.8\end{array}$ & $\begin{array}{r}35.7 \\
62.5 \\
1.8\end{array}$ \\
\hline $\begin{array}{l}\mathrm{SiO} 02 \\
\mathrm{~T} 102 \\
\mathrm{~A} 1203 \\
\mathrm{Fe} 203 \\
\mathrm{MgO} \\
\mathrm{CaO} \\
\mathrm{BaO} \\
\mathrm{Na} 20 \\
\mathrm{~K} 20\end{array}$ & $\begin{array}{c}76.8 \\
0.15 \\
12.6 \\
0.95 \\
0.00 \\
0.29 \\
0.00 \\
4.44 \\
4.83\end{array}$ & $\begin{array}{c}76.2 \\
0.19 \\
12.9 \\
0.93 \\
0.00 \\
0.27 \\
0.00 \\
4.80 \\
4.66\end{array}$ & $\begin{array}{c}\text { oxides } \operatorname{Re} \\
76.8 \\
0.16 \\
12.4 \\
0.94 \\
0.00 \\
0.26 \\
0.00 \\
4.69 \\
4.77\end{array}$ & $\begin{array}{c}\text { ?culate } \\
76.4 \\
0.17 \\
12.8 \\
0.93 \\
0.00 \\
0.27 \\
0.00 \\
4.87 \\
4.58\end{array}$ & $\begin{array}{c}\text { to } 100 \% \\
76.5 \\
0.14 \\
12.8 \\
0.73 \\
0.00 \\
0.28 \\
0.00 \\
4.96 \\
4.61\end{array}$ & $\begin{array}{c}76.8 \\
0.10 \\
12.6 \\
0.85 \\
0.00 \\
0.28 \\
0.00 \\
4.77 \\
4.58\end{array}$ & $\begin{array}{c}77.1 \\
0.14 \\
12.6 \\
0.89 \\
0.00 \\
0.25 \\
0.00 \\
4.84 \\
4.22\end{array}$ & $\begin{array}{c}77.9 \\
0.11 \\
12.1 \\
0.69 \\
0.00 \\
0.25 \\
0.00 \\
4.82 \\
4.18\end{array}$ \\
\hline
\end{tabular}

$\overline{a \text { Unit symbols }}$ defined in Table I. 
APPENDIX E (cont)

Drill Hole USW G-2

\begin{tabular}{|c|c|c|c|c|c|c|c|c|}
\hline $\begin{array}{l}\text { Depth } \\
\text { (ft) } \\
\text { Unit }\end{array}$ & $\begin{array}{l}331 \\
\text { Tpy }\end{array}$ & $\begin{array}{r}358 \\
\text { Tb }\end{array}$ & 358 & 358 & 358 & 358 & $\begin{array}{l}547 \\
T D D\end{array}$ & $\begin{array}{l}547 \\
\text { TDD }\end{array}$ \\
\hline $\begin{array}{l}\mathrm{SiO} 2 \\
\mathrm{TiO} 2 \\
\mathrm{Al} 203 \\
\mathrm{Fe} 203 \\
\mathrm{MgO} \\
\mathrm{CaO} \\
\mathrm{BaO} \\
\mathrm{Na} 20 \\
\mathrm{~K} 20\end{array}$ & $\begin{array}{c}72.0 \\
0.16 \\
12.2 \\
0.91 \\
0.00 \\
0.25 \\
0.00 \\
4.43 \\
4.23\end{array}$ & $\begin{array}{c}72.0 \\
0.16 \\
11.7 \\
0.83 \\
0.00 \\
0.19 \\
0.00 \\
3.99 \\
4.22\end{array}$ & $\begin{array}{c}72.5 \\
0.13 \\
11.6 \\
0.77 \\
0.00 \\
0.18 \\
0.00 \\
4.33 \\
4.22\end{array}$ & $\begin{array}{c}74.1 \\
0.10 \\
11.8 \\
0.62 \\
0.00 \\
0.21 \\
0.00 \\
4.40 \\
3.99\end{array}$ & $\begin{array}{c}73.2 \\
0.13 \\
11.9 \\
0.66 \\
0.00 \\
0.24 \\
0.00 \\
4.54 \\
3.95\end{array}$ & $\begin{array}{c}73.4 \\
0.11 \\
11.9 \\
0.81 \\
0.00 \\
0.22 \\
0.00 \\
4.38 \\
4.06\end{array}$ & $\begin{array}{c}71.1 \\
0.13 \\
11.8 \\
0.85 \\
0.00 \\
0.56 \\
0.00 \\
3.24 \\
4.71\end{array}$ & $\begin{array}{c}73.0 \\
0.09 \\
11.9 \\
0.73 \\
0.00 \\
0.54 \\
0.00 \\
3.36 \\
4.84\end{array}$ \\
\hline Total & 94.2 & 93.1 & 93.7 & 95.2 & 94.6 & 94.8 & 92.3 & 94.5 \\
\hline $\begin{array}{l}\mathrm{Si} \\
\mathrm{Ti} \\
\mathrm{Al} \\
\mathrm{Fe} \\
\mathrm{Mg} \\
\mathrm{Ca} \\
\mathrm{Ba} \\
\mathrm{Na} \\
\mathrm{K}\end{array}$ & $\begin{array}{c}71.0 \\
0.12 \\
14.2 \\
0.68 \\
0.00 \\
0.26 \\
0.00 \\
8.47 \\
5.32\end{array}$ & $\begin{array}{c}72.1 \\
0.12 \\
13.8 \\
0.63 \\
0.00 \\
0.20 \\
0.00 \\
7.75 \\
5.39\end{array}$ & $\begin{array}{c}\text { Ca } \\
71.9 \\
0.10 \\
13.6 \\
0.57 \\
0.00 \\
0.19 \\
0.00 \\
8.33 \\
5.34\end{array}$ & $\begin{array}{c}\text { on Perc } \\
72.3 \\
0.07 \\
13.6 \\
0.46 \\
0.00 \\
0.22 \\
0.00 \\
8.33 \\
4.97\end{array}$ & $\begin{array}{l}\mathrm{t} \\
71.8 \\
0.10 \\
13.7 \\
0.49 \\
0.00 \\
0.25 \\
0.00 \\
8.64 \\
4.94\end{array}$ & $\begin{array}{c}72.0 \\
0.08 \\
13.7 \\
0.60 \\
0.00 \\
0.23 \\
0.00 \\
8.33 \\
5.08\end{array}$ & $\begin{array}{c}72.1 \\
0.10 \\
14.1 \\
0.65 \\
0.00 \\
0.61 \\
0.00 \\
6.37 \\
6.10\end{array}$ & $\begin{array}{c}72.3 \\
0.07 \\
14.0 \\
0.54 \\
0.00 \\
0.57 \\
0.00 \\
6.45 \\
6.12\end{array}$ \\
\hline \multicolumn{9}{|c|}{$\mathrm{Si} /(\mathrm{A})+\mathrm{Fe})$} \\
\hline \multicolumn{9}{|c|}{$(\mathrm{A}]+\mathrm{Fe}) /(2 \mathrm{Mg}+2 \mathrm{C} a+2 \mathrm{Ba}+\mathrm{Na}+\mathrm{K})$} \\
\hline $\begin{array}{l}\mathrm{K} \\
\mathrm{Na} \\
\mathrm{Ca}+\mathrm{Mg}\end{array}$ & $\begin{array}{r}37.9 \\
60.3 \\
1.9\end{array}$ & $\begin{array}{r}40.4 \\
58.1 \\
1.5\end{array}$ & $\begin{array}{c}\text { Mol\% Exc } \\
38.5 \\
60.1 \\
1.4\end{array}$ & $\begin{array}{c}\text { ngeabl } \\
36.8 \\
61.6 \\
1.6\end{array}$ & $\begin{array}{c}\text { ations } \\
35.7 \\
62.4 \\
1.8\end{array}$ & $\begin{array}{r}37.2 \\
61.1 \\
1.7\end{array}$ & $\begin{array}{r}46 . \dot{6} \\
48.7 \\
4.7\end{array}$ & $\begin{array}{r}46.5 \\
49.1 \\
4.4\end{array}$ \\
\hline $\begin{array}{l}\mathrm{SiO} 2 \\
\mathrm{TiO} 2 \\
\mathrm{Al} 203 \\
\mathrm{Fe} 203 \\
\mathrm{MgO} \\
\mathrm{CaO} \\
\mathrm{BaO} \\
\mathrm{Na} 20 \\
\mathrm{~K} 20\end{array}$ & $\begin{array}{c}76.4 \\
0.17 \\
12.9 \\
0.97 \\
0.00 \\
0.27 \\
0.00 \\
4.70 \\
4.49\end{array}$ & $\begin{array}{c}77.4 \\
0.17 \\
12.5 \\
0.89 \\
0.00 \\
0.20 \\
0.00 \\
4.29 \\
4.53\end{array}$ & $\begin{array}{c}0 \times i d e s \\
77.3 \\
0.14 \\
12.4 \\
0.82 \\
0.00 \\
0.19 \\
0.00 \\
4.62 \\
4.50\end{array}$ & $\begin{array}{c}\text { alcula } \\
77.8 \\
0.11 \\
12.4 \\
0.65 \\
0.00 \\
0.22 \\
0.00 \\
4.62 \\
4.19\end{array}$ & $\begin{array}{c}\text { to } 100 \% \\
77.4 \\
0.14 \\
12.5 \\
0.70 \\
0.00 \\
0.25 \\
0.00 \\
4.80 \\
4.17\end{array}$ & $\begin{array}{c}77.4 \\
0.12 \\
12.5 \\
0.85 \\
0.00 \\
0.23 \\
0.00 \\
4.62 \\
4.28\end{array}$ & $\begin{array}{c}77.0 \\
0.14 \\
12.7 \\
0.92 \\
0.00 \\
0.61 \\
0.00 \\
3.51 \\
5.10\end{array}$ & $\begin{array}{c}77.2 \\
0.10 \\
12.6 \\
0.77 \\
0.00 \\
0.57 \\
0.00 \\
3.56 \\
5.12\end{array}$ \\
\hline
\end{tabular}




\section{APPENDIX E (cont)}

Drill Hole USW G-2

\begin{tabular}{|c|c|}
\hline $\begin{array}{l}\text { Depth } \\
\text { (ft) } \\
\text { Unit }\end{array}$ & $\begin{array}{l}547 \\
T p p\end{array}$ \\
\hline $\begin{array}{l}\mathrm{SiO22} \\
\mathrm{Ti02} \\
\mathrm{Al} 203 \\
\mathrm{Fe} 203 \\
\mathrm{MgO} \\
\mathrm{CaO} \\
\mathrm{BaO} \\
\mathrm{Na2O} \\
\mathrm{K} 20\end{array}$ & $\begin{array}{c}72.2 \\
0.09 \\
12.2 \\
0.76 \\
0.00 \\
0.51 \\
0.00 \\
3.67 \\
5.07\end{array}$ \\
\hline Total & 94.5 \\
\hline $\begin{array}{l}\text { Cation } \\
\mathrm{Si} \\
\mathrm{Ti} \\
\mathrm{Al} \\
\mathrm{Fe} \\
\mathrm{Mg} \\
\mathrm{Ca} \\
\mathrm{Ba} \\
\mathrm{Na} \\
\mathrm{K}\end{array}$ & $\begin{array}{c}\text { Percent } \\
71.3 \\
0.07 \\
14.1 \\
0.56 \\
0.00 \\
0.54 \\
0.00 \\
7.02 \\
6.39\end{array}$ \\
\hline $\mathrm{Si} / \mathrm{A}$ & $\begin{array}{l}(\mathrm{A} 1+\mathrm{Fe}) \\
4.84\end{array}$ \\
\hline$(\mathrm{A})+\mathrm{Fe})$ & $\begin{array}{l}\mathrm{H} /(2 \mathrm{Mg}+2 \mathrm{Ca}+2 \mathrm{Ba}+\mathrm{Na}+\mathrm{K}) \\
\quad 1.02\end{array}$ \\
\hline $\begin{array}{l}\text { Mol\% Ex } \\
\mathrm{K} \\
\mathrm{Na} \\
\mathrm{Ca}+\mathrm{Mg}\end{array}$ & $\begin{array}{l}\text { xchangeable Cations } \\
45.8 \\
50.4 \\
3.9\end{array}$ \\
\hline $\begin{array}{l}\text { Oxides } \\
\text { Si02 } \\
\text { Ti02 } \\
\text { Al203 } \\
\text { Fe203 } \\
\text { MgO } \\
\mathrm{CaO} \\
\mathrm{BaO} \\
\mathrm{Na} 20 \\
\mathrm{~K} 2 \mathrm{O}\end{array}$ & $\begin{array}{l}\text { Recalculated to } 100 \% \\
76.4 \\
0.10 \\
12.9 \\
0.80 \\
0.00 \\
0.54 \\
0.00 \\
3.89 \\
5.37\end{array}$ \\
\hline
\end{tabular}


APPENDIX E (cont)

Drill Hole USW GU-3

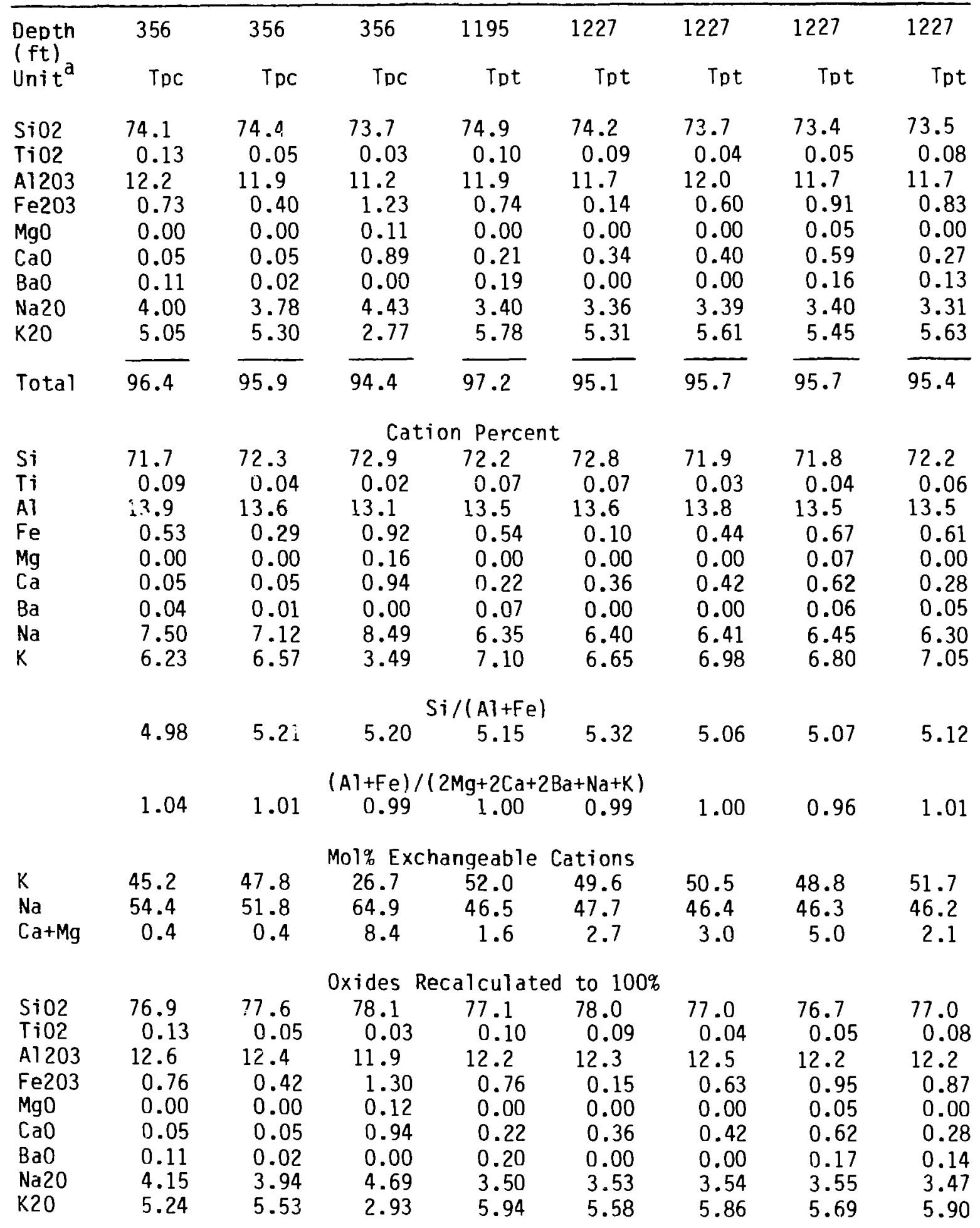


Drill Hole USW GU-3

\begin{tabular}{|c|c|c|c|c|c|c|c|c|}
\hline $\begin{array}{l}\text { Depth } \\
(\mathrm{ft}) \\
\text { Unit }^{\mathrm{a}}\end{array}$ & $\begin{array}{r}1227 \\
\text { Tpt }\end{array}$ & $\begin{array}{r}1227 \\
\text { Tot }\end{array}$ & $\begin{array}{r}1227 \\
\text { Tpt }\end{array}$ & $\begin{array}{r}1227 \\
\text { Tpt }\end{array}$ & $\begin{array}{r}1227 \\
\text { Tpt }\end{array}$ & $\begin{array}{r}1303 \\
\text { Tpt }\end{array}$ & $\begin{array}{r}1303 \\
\text { Tpt }\end{array}$ & $\begin{array}{r}1303 \\
\text { Tpt }\end{array}$ \\
\hline $\begin{array}{l}\mathrm{SiO} 2 \\
\mathrm{TiO} 2 \\
\mathrm{~A} 1203 \\
\mathrm{Fe} 203 \\
\mathrm{MgO} \\
\mathrm{CaO} \\
\mathrm{BaO} \\
\mathrm{Na} 20 \\
\mathrm{~K} 20\end{array}$ & $\begin{array}{c}74.1 \\
0.11 \\
11.8 \\
0.73 \\
0.00 \\
0.24 \\
0.07 \\
3.41 \\
5.55\end{array}$ & $\begin{array}{c}74.4 \\
0.09 \\
11.5 \\
0.81 \\
0.00 \\
0.22 \\
0.04 \\
3.27 \\
5.58\end{array}$ & $\begin{array}{c}74.0 \\
0.08 \\
11.8 \\
0.95 \\
0.00 \\
0.37 \\
0.00 \\
3.24 \\
5.50\end{array}$ & $\begin{array}{l}73.8 \\
0.07 \\
11.7 \\
0.46 \\
0.01 \\
0.52 \\
0.13 \\
3.13 \\
5.72\end{array}$ & $\begin{array}{c}74.2 \\
0.04 \\
11.7 \\
0.20 \\
0.00 \\
0.31 \\
0.00 \\
3.47 \\
5.58\end{array}$ & $\begin{array}{c}74.6 \\
0.09 \\
11.9 \\
0.77 \\
0.02 \\
0.29 \\
0.00 \\
3.42 \\
5.59\end{array}$ & $\begin{array}{c}75.4 \\
0.01 \\
11.6 \\
0.38 \\
0.00 \\
0.36 \\
0.00 \\
3.32 \\
5.50\end{array}$ & $\begin{array}{c}73.4 \\
0.04 \\
12.0 \\
0.69 \\
0.04 \\
0.32 \\
0.12 \\
3.39 \\
5.49\end{array}$ \\
\hline Total & 96.0 & 96.0 & 95.9 & 95.5 & 95.5 & 96.7 & 96.5 & 95.5 \\
\hline $\begin{array}{l}\mathrm{Si} \\
\mathrm{Ti} \\
\mathrm{Al} \\
\mathrm{Fe} \\
\mathrm{Mg} \\
\mathrm{Ca} \\
\mathrm{Ba} \\
\mathrm{Na} \\
\mathrm{Ka}\end{array}$ & $\begin{array}{c}72.2 \\
0.08 \\
13.6 \\
0.54 \\
0.00 \\
0.25 \\
0.03 \\
6.44 \\
6.90\end{array}$ & $\begin{array}{c}72.7 \\
0.07 \\
13.3 \\
0.60 \\
0.00 \\
0.23 \\
0.02 \\
6.19 \\
6.95\end{array}$ & $\begin{array}{c}72.2^{C a} \\
0.06 \\
13.6 \\
0.70 \\
0.00 \\
0.39 \\
0.00 \\
6.14 \\
6.85\end{array}$ & $\begin{array}{c}\text { on Per } \\
72.4 \\
0.05 \\
13.5 \\
0.34 \\
0.01 \\
0.55 \\
0.05 \\
5.95 \\
7.16\end{array}$ & $\begin{array}{c}72.5 \\
0.03 \\
13.5 \\
0.15 \\
0.00 \\
0.32 \\
0.00 \\
6.57 \\
6.95\end{array}$ & $\begin{array}{c}72.2 \\
0.07 \\
13.5 \\
0.56 \\
0.03 \\
0.30 \\
0.00 \\
6.41 \\
6.90\end{array}$ & $\begin{array}{c}73.1 \\
0.01 \\
13.2 \\
0.28 \\
0.00 \\
0.37 \\
0.00 \\
6.24 \\
6.80\end{array}$ & $\begin{array}{c}71.9 \\
0.03 \\
13.8 \\
0.51 \\
0.06 \\
0.34 \\
0.05 \\
6.44 \\
6.86\end{array}$ \\
\hline \multicolumn{9}{|c|}{$\mathrm{Si} /(\mathrm{Al}+\mathrm{Fe})$} \\
\hline \multicolumn{9}{|c|}{$(\mathrm{A} 1+\mathrm{Fe}) /(2 \mathrm{Mg}+2 \mathrm{Ca}+2 \mathrm{Ba}+\mathrm{Na}+\mathrm{K})$} \\
\hline $\begin{array}{l}\mathrm{K} \\
\mathrm{Na} \\
\mathrm{Ca}+\mathrm{Mg}\end{array}$ & $\begin{array}{r}50.8 \\
47.4 \\
1.8\end{array}$ & $\begin{array}{r}52.0 \\
46.3 \\
1.7\end{array}$ & $\begin{array}{c}101 \% \text { Exc } \\
51.2 \\
45.9 \\
2.9\end{array}$ & $\begin{array}{c}\text { ngeabl } \\
52.4 \\
43.5 \\
4.1\end{array}$ & $\begin{array}{c}\text { ations } \\
50.2 \\
47.5 \\
2.3\end{array}$ & $\begin{array}{r}50.6 \\
47.0 \\
2.4\end{array}$ & $\begin{array}{r}50.7 \\
46.5 \\
2.8\end{array}$ & $\begin{array}{r}50.1 \\
47.0 \\
2.9\end{array}$ \\
\hline $\begin{array}{l}\mathrm{SiO} 02 \\
\mathrm{TiO} 02 \\
\mathrm{~A} 1203 \\
\mathrm{Fe} 203 \\
\mathrm{MgO} \\
\mathrm{CaO} \\
\mathrm{BaO} \\
\mathrm{Na} 20 \\
\mathrm{~K} 20\end{array}$ & $\begin{array}{c}77.2 \\
0.11 \\
12.3 \\
0.76 \\
0.00 \\
0.25 \\
0.07 \\
3.55 \\
5.78\end{array}$ & $\begin{array}{c}77.5 \\
0.09 \\
12.0 \\
0.84 \\
0.00 \\
0.23 \\
0.14 \\
3.41 \\
5.81\end{array}$ & $\begin{array}{c}\text { Xides } \\
77.1 \\
0.08 \\
12.3 \\
0.99 \\
0.00 \\
0.39 \\
0.00 \\
3.38 \\
5.73\end{array}$ & $\begin{array}{c}\text { alcula } \\
77.3 \\
0.07 \\
12.2 \\
0.48 \\
0.01 \\
0.54 \\
0.14 \\
3.28 \\
5.99\end{array}$ & $\begin{array}{c}\text { to } 100 \\
77.7 \\
0.04 \\
12.3 \\
0.21 \\
0.00 \\
0.32 \\
0.00 \\
3.63 \\
5.84\end{array}$ & $\begin{array}{c}77.2 \\
0.09 \\
12.3 \\
0.80 \\
0.02 \\
0.30 \\
0.00 \\
3.54 \\
5.78\end{array}$ & $\begin{array}{c}78.1 \\
0.01 \\
12.0 \\
0.39 \\
0.00 \\
0.37 \\
0.00 \\
3.44 \\
5.70\end{array}$ & $\begin{array}{c}76.9 \\
0.04 \\
12.5 \\
0.72 \\
0.04 \\
0.34 \\
0.13 \\
3.55 \\
5.75\end{array}$ \\
\hline
\end{tabular}


APPENDIX E (cont)

Drill Hole USW GU-3

\begin{tabular}{|c|c|c|c|c|c|c|c|c|}
\hline $\begin{array}{l}\text { Depth } \\
\text { (ft) } \\
\text { Unit }\end{array}$ & $\begin{array}{r}1303 \\
\text { Tpt }\end{array}$ & $\begin{array}{r}1303 \\
\text { Tpt }\end{array}$ & $\begin{array}{r}1303 \\
\text { Tot }\end{array}$ & $\begin{array}{r}1394 \\
\text { Tpt }\end{array}$ & $\begin{array}{r}1394 \\
\text { Tpt }\end{array}$ & $\begin{array}{r}1394 \\
\text { Tpt }\end{array}$ & $\begin{array}{r}1537 \\
\text { Tht }\end{array}$ & $\begin{array}{r}1537 \\
\text { Tnt }\end{array}$ \\
\hline $\begin{array}{l}\mathrm{SiO} 2 \\
\mathrm{Ti02} \\
\mathrm{Al} 2.03 \\
\mathrm{Fe} 203 \\
\mathrm{MgO} \\
\mathrm{CaO} \\
\mathrm{BaO} \\
\mathrm{Na} 20 \\
\mathrm{~K} 20\end{array}$ & $\begin{array}{c}74.1 \\
0.04 \\
12.1 \\
0.66 \\
0.00 \\
0.34 \\
0.00 \\
3.58 \\
5.66\end{array}$ & $\begin{array}{c}74.4 \\
0.07 \\
11.9 \\
0.55 \\
0.00 \\
0.29 \\
0.12 \\
3.44 \\
5.66\end{array}$ & $\begin{array}{c}74.1 \\
0.03 \\
11.7 \\
0.54 \\
0.00 \\
0.36 \\
0.00 \\
3.20 \\
5.68\end{array}$ & $\begin{array}{c}73.9 \\
0.00 \\
11.8 \\
0.85 \\
0.02 \\
0.43 \\
0.00 \\
3.49 \\
4.93\end{array}$ & $\begin{array}{c}72.4 \\
0.05 \\
11.7 \\
0.80 \\
0.02 \\
0.43 \\
0.00 \\
3.41 \\
4.89\end{array}$ & $\begin{array}{c}73.6 \\
0.05 \\
12.0 \\
0.78 \\
0.00 \\
0.43 \\
0.06 \\
3.69 \\
4.80\end{array}$ & $\begin{array}{c}74.5 \\
0.01 \\
12.1 \\
0.63 \\
0.00 \\
0.60 \\
0.75 \\
3.21 \\
4.92\end{array}$ & $\begin{array}{c}73.9 \\
0.07 \\
11.8 \\
0.71 \\
0.00 \\
0.57 \\
0.00 \\
3.06 \\
5.13\end{array}$ \\
\hline Total & 96.5 & 96.5 & 95.6 & 95.4 & 93.7 & 95.4 & 96.7 & 95.3 \\
\hline $\begin{array}{l}\mathrm{Si} \\
\mathrm{Ti} \\
\mathrm{Al} \\
\mathrm{Fe} \\
\mathrm{Mg} \\
\mathrm{Ca} \\
\mathrm{Ba} \\
\mathrm{Na} \\
\mathrm{K}\end{array}$ & $\begin{array}{c}71.6 \\
0.03 \\
13.8 \\
0.48 \\
0.00 \\
0.35 \\
0.00 \\
6.71 \\
6.98\end{array}$ & $\begin{array}{c}72.1 \\
0.05 \\
13.6 \\
0.40 \\
0.00 \\
0.30 \\
0.05 \\
6.46 \\
7.00\end{array}$ & $\begin{array}{c}\text { Cat } \\
72.6 \\
0.02 \\
13.5 \\
0.40 \\
0.00 \\
0.38 \\
0.00 \\
6.07 \\
7.09\end{array}$ & $\begin{array}{c}\text { n Perc } \\
72.4 \\
0.00 \\
13.6 \\
0.63 \\
0.03 \\
0.45 \\
0.00 \\
6.64 \\
6.17\end{array}$ & $\begin{array}{c}72.3 \\
0.04 \\
13.7 \\
0.60 \\
0.03 \\
0.46 \\
0.00 \\
6.60 \\
6.23\end{array}$ & $\begin{array}{c}72.1 \\
0.04 \\
13.8 \\
0.57 \\
0.00 \\
0.45 \\
0.02 \\
7.01 \\
6.00\end{array}$ & $\begin{array}{c}72.5 \\
0.01 \\
13.9 \\
0.46 \\
0.00 \\
0.63 \\
0.29 \\
6.06 \\
6.11\end{array}$ & $\begin{array}{c}72.8 \\
0.05 \\
13.7 \\
0.53 \\
0.00 \\
0.60 \\
0.00 \\
5.84 \\
6.44\end{array}$ \\
\hline \multicolumn{9}{|c|}{$\mathrm{Si} /(\mathrm{A} l+\mathrm{Fe})$} \\
\hline \multicolumn{9}{|c|}{$(\mathrm{A} 1+\mathrm{Fe}) /(2 \mathrm{Mg}+2 \mathrm{Ca}+2 \mathrm{Ba}+\mathrm{Na}+\mathrm{K})$} \\
\hline $\begin{array}{l}\mathrm{K} \\
\mathrm{Na} \\
\mathrm{Ca}+\mathrm{Mg}\end{array}$ & $\begin{array}{r}49.7 \\
47.8 \\
2.5\end{array}$ & $\begin{array}{r}50.8 \\
47.0 \\
2.2\end{array}$ & $\begin{array}{c}\text { Mol\% Ex } \\
52.4 \\
44.8 \\
2.8\end{array}$ & $\begin{array}{c}\text { ngeabi } 1 \\
46.4 \\
50.0 \\
3.6\end{array}$ & $\begin{array}{c}\text { Cations } \\
46.8 \\
49.6 \\
3.7\end{array}$ & $\begin{array}{r}44.6 \\
52.1 \\
3.4\end{array}$ & $\begin{array}{r}47.8 \\
47.4 \\
4.9\end{array}$ & $\begin{array}{r}50.0 \\
45.3 \\
4.7\end{array}$ \\
\hline $\begin{array}{l}\mathrm{SiO} 2 \\
\mathrm{TiO} 2 \\
11203 \\
\mathrm{Fe} 203 \\
\mathrm{MgO} \\
\mathrm{CaO} \\
\mathrm{BaO} \\
\mathrm{Na} 20 \\
\mathrm{~K} 20\end{array}$ & $\begin{array}{c}76.8 \\
0.04 \\
12.6 \\
0.68 \\
0.00 \\
0.35 \\
0.00 \\
3.71 \\
5.86\end{array}$ & $\begin{array}{c}77.1 \\
0.07 \\
12.4 \\
0.57 \\
0.00 \\
0.30 \\
0.12 \\
3.56 \\
5.87\end{array}$ & $\begin{array}{c}0 \times i d e s \\
77.5 \\
0.03 \\
12.2 \\
0.56 \\
0.00 \\
0.38 \\
0.00 \\
3.35 \\
5.94\end{array}$ & $\begin{array}{c}\text { alcula } \\
77.4 \\
0.00 \\
12.4 \\
0.89 \\
0.02 \\
0.45 \\
0.00 \\
3.66 \\
5.17\end{array}$ & $\begin{array}{c}\text { to } 100 \\
77.3 \\
0.05 \\
12.4 \\
0.85 \\
0.02 \\
0.46 \\
0.00 \\
3.64 \\
5.22\end{array}$ & $\begin{array}{c}77.2 \\
0.05 \\
12.5 \\
0.82 \\
0.00 \\
0.45 \\
0.06 \\
3.87 \\
5.03\end{array}$ & $\begin{array}{c}77.0 \\
0.01 \\
12.5 \\
0.65 \\
0.00 \\
0.62 \\
0.78 \\
3.32 \\
5.09\end{array}$ & $\begin{array}{c}77.6 \\
0.07 \\
12.4 \\
0.74 \\
0.00 \\
0.60 \\
0.00 \\
3.21 \\
5.38\end{array}$ \\
\hline
\end{tabular}


APPENUIX E (cont)

Drill Hole USW GU-3

\begin{tabular}{|c|c|c|c|c|}
\hline $\begin{array}{l}\text { Depth } \\
\text { (ft) } \\
\text { Unit }\end{array}$ & $\begin{array}{r}1537 \\
\text { Tht }\end{array}$ & Tht & 1537 & $\begin{array}{l}1571 \\
\text { Tip }\end{array}$ \\
\hline $\begin{array}{l}\mathrm{SiO} 2 \\
\mathrm{TiO} 02 \\
\mathrm{~A} 1203 \\
\mathrm{Fe} 203 \\
\mathrm{MgO} \\
\mathrm{CaO} \\
\mathrm{BaO} \\
\mathrm{Na20} \\
\mathrm{K} 20\end{array}$ & $\begin{array}{c}73.9 \\
0.07 \\
11.8 \\
0.55 \\
0.00 \\
0.60 \\
0.00 \\
3.13 \\
5.00\end{array}$ & $\begin{array}{c}73.1 \\
0.04 \\
12.1 \\
0.58 \\
0.00 \\
0.52 \\
0.00 \\
3.38 \\
5.05\end{array}$ & $\begin{array}{c}73.9 \\
0.07 \\
11.8 \\
0.71 \\
0.00 \\
0.57 \\
0.00 \\
3.06 \\
5.13\end{array}$ & $\begin{array}{c}74.6 \\
0.05 \\
11.9 \\
0.75 \\
0.00 \\
0.40 \\
1.57 \\
3.35 \\
4.86\end{array}$ \\
\hline Total & 95.1 & 94.8 & 95.3 & 97.5 \\
\hline $\begin{array}{l}\mathrm{Si} \\
\mathrm{Ti} \\
\mathrm{Al} \\
\mathrm{Fa} \\
\mathrm{Mg} \\
\mathrm{Ca} \\
\mathrm{Ba} \\
\mathrm{Na} \\
\mathrm{K}\end{array}$ & $\begin{array}{c}\quad{ }^{C a} \\
72.9^{-} \\
0.05 \\
13.7 \\
0.41 \\
0.00 \\
0.63 \\
0.00 \\
5.98 \\
6.29\end{array}$ & $\begin{array}{c}\text { on Perc } \\
72.1 \\
0.03 \\
14.0 \\
0.43 \\
0.00 \\
0.55 \\
0.00 \\
6.46 \\
6.35\end{array}$ & $\begin{array}{c}\text { t } \\
72.8 \\
0.05 \\
13.7 \\
0.53 \\
0.00 \\
0.60 \\
0.00 \\
5.84 \\
6.44\end{array}$ & $\begin{array}{c}72.5 \\
0.04 \\
13.6 \\
0.55 \\
0.00 \\
0.42 \\
0.60 \\
6.31 \\
6.02\end{array}$ \\
\hline \multicolumn{5}{|c|}{$\mathrm{Si} /(\mathrm{Al}+\mathrm{Fe})$} \\
\hline & $\begin{array}{c}(\mathrm{A}]+\mathrm{Fe}) / \\
1.04\end{array}$ & $\begin{array}{c}\mathrm{Mg}+2 \mathrm{Cat} \\
1.04\end{array}$ & $\begin{array}{c}(3 a+N a+K) \\
1.06\end{array}$ & 0.98 \\
\hline $\begin{array}{l}\mathrm{K} \\
\mathrm{Na} \\
\mathrm{Ca}+\mathrm{Mg}\end{array}$ & $\begin{array}{c}\text { Mol\% Exc } \\
48.7 \\
46.4 \\
4.9\end{array}$ & $\begin{array}{c}\text { ngeable } \\
47.5 \\
48.4 \\
4.1\end{array}$ & $\begin{array}{c}\text { Cations } \\
50.0 \\
45.3 \\
4.7\end{array}$ & $\begin{array}{r}47.2 \\
49.5 \\
3.3\end{array}$ \\
\hline $\begin{array}{l}\mathrm{SiO} 02 \\
\mathrm{TiO} 2 \\
\mathrm{~A} 1203 \\
\mathrm{Fe} 203 \\
\mathrm{MgO} \\
\mathrm{CaO} \\
\mathrm{BaO} \\
\mathrm{Na} 20 \\
\mathrm{~K} 20\end{array}$ & $\begin{array}{c}\text { Oxides Re } \\
77.7 \\
0.07 \\
12.4 \\
0.58 \\
0.00 \\
0.63 \\
0.00 \\
3.29 \\
5.26\end{array}$ & $\begin{array}{c}\text { 1culate } \\
77.1 \\
0.04 \\
12.7 \\
0.51 \\
0.00 \\
0.55 \\
0.00 \\
3.57 \\
5.33\end{array}$ & $\begin{array}{c}\text { to } 100 \% \\
77.6 \\
0.07 \\
12.4 \\
0.74 \\
0.00 \\
0.60 \\
0.00 \\
3.21 \\
5.38\end{array}$ & $\begin{array}{c}76.5 \\
0.05 \\
12.2 \\
0.77 \\
0.00 \\
0.41 \\
1.61 \\
3.44 \\
4.99\end{array}$ \\
\hline
\end{tabular}


APPENDIX E (cont)

Drill Hole USW G-4

\begin{tabular}{|c|c|c|c|}
\hline $\begin{array}{l}\text { Depth } \\
(\mathrm{ft}) \\
\text { Unit }\end{array}$ & $\begin{array}{r}1432 \\
\text { Tht }\end{array}$ & $\begin{array}{r}1432 \\
\text { Tht }\end{array}$ & $\begin{array}{r}1432 \\
\text { Tht }\end{array}$ \\
\hline $\begin{array}{l}\mathrm{SiO} 02 \\
\mathrm{Ti} 02 \\
\mathrm{Al} 203 \\
\mathrm{Fe} 203 \\
\mathrm{MgO} \\
\mathrm{CaO} \\
\mathrm{BaO} \\
\mathrm{Na} 20 \\
\mathrm{~K} 20\end{array}$ & $\begin{array}{c}73.7 \\
0.11 \\
11.9 \\
0.69 \\
0.02 \\
0.49 \\
0.00 \\
3.38 \\
4.98\end{array}$ & $\begin{array}{c}74.3 \\
0.06 \\
11.9 \\
0.74 \\
0.07 \\
0.53 \\
0.18 \\
3.35 \\
5.01\end{array}$ & $\begin{array}{c}73.8 \\
0.07 \\
11.7 \\
0.79 \\
0.05 \\
0.44 \\
0.00 \\
3.33 \\
4.99\end{array}$ \\
\hline Total & 95.3 & 96.2 & 95.2 \\
\hline $\begin{array}{l}\mathrm{Si} \\
\mathrm{Ti} \\
\mathrm{Al} \\
\mathrm{Fe} \\
\mathrm{Mg} \\
\mathrm{CA} \\
\mathrm{BA} \\
\mathrm{NA} \\
\mathrm{K}\end{array}$ & $\begin{array}{c}\text { Cation } \\
72.4 \\
0.08 \\
13.8 \\
0.51 \\
0.03 \\
0.52 \\
0.00 \\
6.43 \\
6.24\end{array}$ & $\begin{array}{c}\text { Percent } \\
72.4 \\
0.04 \\
13.7 \\
0.54 \\
0.10 \\
0.55 \\
0.07 \\
6.33 \\
6.23\end{array}$ & $\begin{array}{c}72.6 \\
0.05 \\
13.6 \\
0.59 \\
0.07 \\
0.46 \\
0.00 \\
6.35 \\
6.27\end{array}$ \\
\hline \multicolumn{4}{|c|}{$\mathrm{Si} /(\mathrm{Al}+\mathrm{Fe})$} \\
\hline \multicolumn{3}{|c|}{$(\mathrm{Al}+\mathrm{Fe}) /(2 \mathrm{Mg}+2 \mathrm{Ca}+2 \mathrm{Ba}+\mathrm{Na}+\mathrm{K})$} & $\begin{array}{r}(a+K) \\
1.04\end{array}$ \\
\hline $\begin{array}{l}\mathrm{Mol} \% \\
\mathrm{Ka} \\
\mathrm{Na}+\mathrm{Mg}\end{array}$ & $\begin{array}{c}\text { Exchang } \\
47.2 \\
48.7 \\
4.1\end{array}$ & $\begin{array}{c}\text { eable Cat } \\
47.1 \\
47.9 \\
5.0\end{array}$ & $\begin{array}{l}\text { ions } \\
47.6 \\
48.3 \\
4.1\end{array}$ \\
\hline $\begin{array}{l}\text { Oxide } \\
\text { Si02 } \\
\text { Ti02 } \\
\text { A1203 } \\
\text { Fe203 } \\
\text { MgO } \\
\text { CaO } \\
\text { BaO } \\
\text { Na20 } \\
\text { K2O }\end{array}$ & $\begin{array}{c}\text { Recalc } \\
77.3 \\
0.12 \\
12.5 \\
0.72 \\
0.02 \\
0.51 \\
0.00 \\
3.55 \\
5.22\end{array}$ & $\begin{array}{c}11 \text { ated to } \\
77.3 \\
0.06 \\
12.4 \\
0.77 \\
0.07 \\
0.55 \\
0.19 \\
3.48 \\
5.21\end{array}$ & $\begin{array}{c}100 \% \\
77.5 \\
0.07 \\
12.3 \\
0.83 \\
0.05 \\
0.46 \\
0.00 \\
3.50 \\
5.24\end{array}$ \\
\hline
\end{tabular}


APPENDIX E (cont)

Drill Hole USW H-4

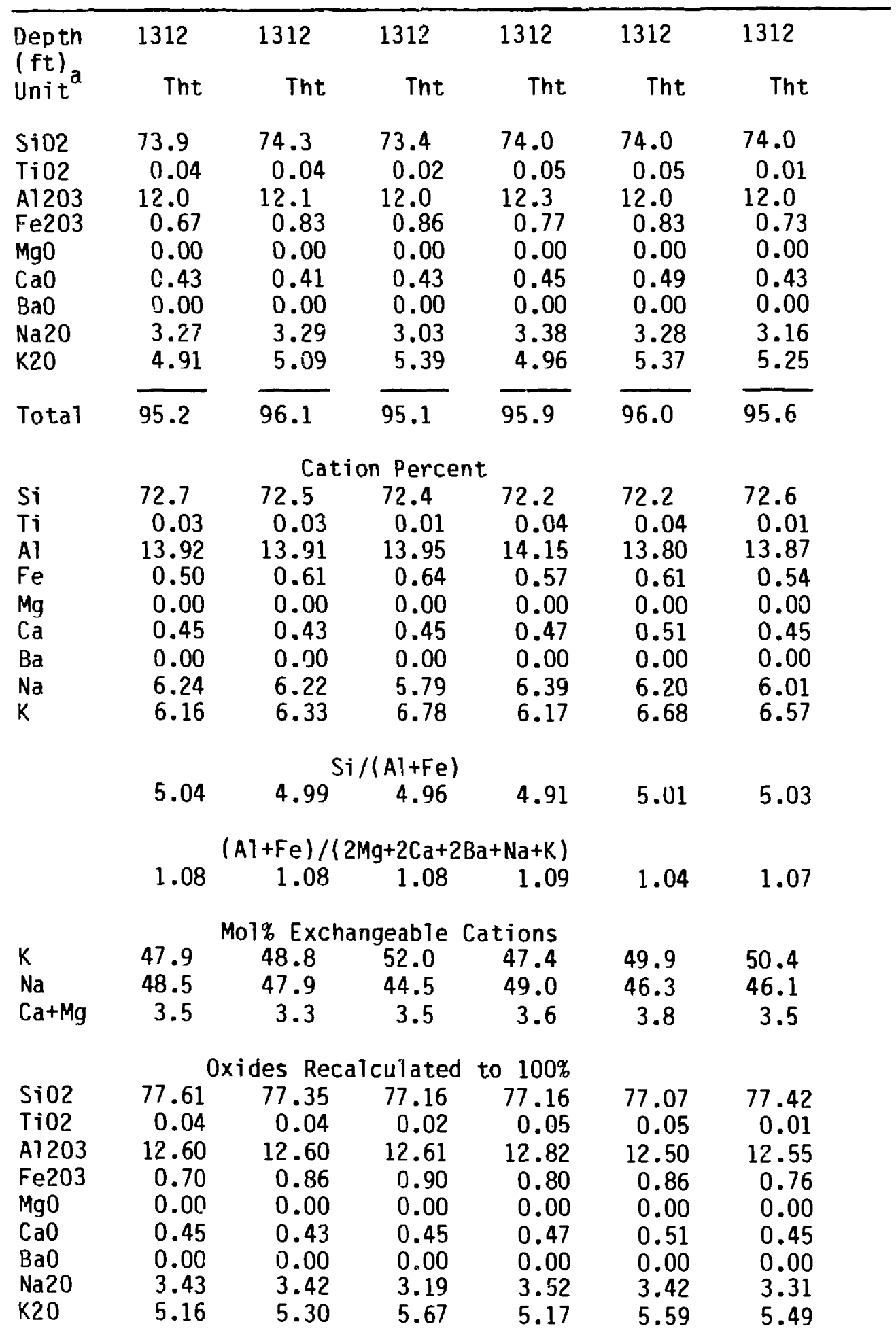


Drill Hole USW H-5

\begin{tabular}{|c|c|c|c|c|c|c|c|c|}
\hline $\begin{array}{l}\text { Depth } \\
(\mathrm{ft})^{\mathrm{a}} \\
\text { Unit }\end{array}$ & $\begin{array}{l}450 \\
T D C\end{array}$ & $\begin{array}{l}450 \\
T p c\end{array}$ & $\begin{array}{l}450 \\
T p c\end{array}$ & $\begin{array}{l}450 \\
T p c\end{array}$ & $\begin{array}{r}1610 \\
\text { Tpt }\end{array}$ & $\begin{array}{r}1762 \\
\text { Tht }\end{array}$ & $\begin{array}{r}1762 \\
\text { Tht }\end{array}$ & $\begin{array}{r}1762 \\
\text { Tht }\end{array}$ \\
\hline $\begin{array}{l}\mathrm{SiO} 2 \\
\mathrm{TiO} 02 \\
\mathrm{Al} 203 \\
\mathrm{Fe} 203 \\
\mathrm{MgO} \\
\mathrm{CaO} \\
\mathrm{BaO} \\
\mathrm{Na} 20 \\
\mathrm{~K} 20\end{array}$ & $\begin{array}{c}74.6 \\
0.09 \\
11.9 \\
0.71 \\
0.00 \\
0.14 \\
0.04 \\
4.21 \\
4.90\end{array}$ & $\begin{array}{c}73.6 \\
0.10 \\
11.8 \\
0.77 \\
0.00 \\
0.18 \\
0.11 \\
4.28 \\
4.66\end{array}$ & $\begin{array}{c}72.3 \\
0.09 \\
11.8 \\
0.62 \\
0.00 \\
0.20 \\
0.20 \\
4.24 \\
4.65\end{array}$ & $\begin{array}{c}73.7 \\
0.05 \\
11.6 \\
0.83 \\
0.01 \\
0.20 \\
0.20 \\
4.34 \\
4.64\end{array}$ & $\begin{array}{c}74.3 \\
0.03 \\
12.0 \\
0.73 \\
0.02 \\
0.45 \\
0.06 \\
3.42 \\
5.05\end{array}$ & $\begin{array}{c}73.7 \\
0.05 \\
11.8 \\
0.76 \\
0.00 \\
0.49 \\
0.00 \\
3.32 \\
4.96\end{array}$ & $\begin{array}{c}73.7 \\
0.05 \\
11.7 \\
0.64 \\
0.00 \\
0.48 \\
0.14 \\
3.52 \\
4.96\end{array}$ & $\begin{array}{c}72.8 \\
0.07 \\
12.0 \\
0.66 \\
0.00 \\
0.48 \\
0.00 \\
3.47 \\
4.75\end{array}$ \\
\hline Total & 96.6 & 95.5 & 94.1 & 95.6 & 96.1 & 95.1 & 95.2 & 94.2 \\
\hline $\begin{array}{l}\mathrm{Si} \\
\mathrm{Ti} \\
\mathrm{Al} \\
\mathrm{Fe} \\
\mathrm{Mg} \\
\mathrm{Ca} \\
\mathrm{Ba} \\
\mathrm{Na} \\
\mathrm{K}\end{array}$ & $\begin{array}{c}71.9 \\
0.07 \\
13.5 \\
0.51 \\
0.00 \\
0.14 \\
0.02 \\
7.86 \\
6.02\end{array}$ & $\begin{array}{c}71.7 \\
0.07 \\
13.5 \\
0.56 \\
0.00 \\
0.19 \\
0.04 \\
8.08 \\
5.79\end{array}$ & $\begin{array}{c} \\
71.4 \\
0.07 \\
13.7 \\
0.46 \\
0.00 \\
0.21 \\
0.08 \\
8.12 \\
5.86\end{array}$ & $\begin{array}{c}\text { ation Pe } \\
71.8 \\
0.04 \\
13.3 \\
0.61 \\
0.01 \\
0.21 \\
0.08 \\
8.20 \\
5.77\end{array}$ & $\begin{array}{c}\text { ent } \\
72.4 \\
0.02 \\
13.8 \\
0.54 \\
0.03 \\
0.47 \\
0.02 \\
6.46 \\
6.28\end{array}$ & $\begin{array}{c}72.6 \\
0.04 \\
13.7 \\
0.56 \\
0.00 \\
0.52 \\
0.00 \\
6.34 \\
6.23\end{array}$ & $\begin{array}{c}72.4 \\
0.04 \\
13.6 \\
0.47 \\
0.00 \\
0.51 \\
0.05 \\
6.71 \\
6.22\end{array}$ & $\begin{array}{c}72.2 \\
0.05 \\
14.0 \\
0.49 \\
0.00 \\
0.51 \\
0.00 \\
6.67 \\
6.01\end{array}$ \\
\hline \multicolumn{9}{|c|}{$\mathrm{Si} /(\mathrm{Al}+\mathrm{Fe})$} \\
\hline \multicolumn{9}{|c|}{$(\mathrm{A})+\mathrm{Fe}) /(2 \mathrm{Mg}+2 \mathrm{Ca}+2 \mathrm{Ba}+\mathrm{Na}+\mathrm{K})$} \\
\hline $\begin{array}{l}\mathrm{K} \\
\mathrm{Na} \\
\mathrm{Ca}+\mathrm{Mg}\end{array}$ & $\begin{array}{r}42.9 \\
56.0 \\
1.0\end{array}$ & $\begin{array}{r}41.2 \\
57.5 \\
1.3\end{array}$ & $\begin{array}{l}\text { Mo1\% E } \\
41.3 \\
57.2 \\
1.5\end{array}$ & $\begin{array}{c}\text { changeab } \\
40.6 \\
57.8 \\
1.6\end{array}$ & $\begin{array}{l}\text { Catior } \\
47.4 \\
48.8 \\
3.8\end{array}$ & $\begin{array}{r}47.6 \\
48.4 \\
4.0\end{array}$ & $\begin{array}{r}46.3 \\
49.9 \\
3.8\end{array}$ & $\begin{array}{r}45.6 \\
50.6 \\
3.9\end{array}$ \\
\hline $\begin{array}{l}\mathrm{SiO} 02 \\
\mathrm{TiD2} \\
\mathrm{A} 1203 \\
\mathrm{Fe} 203 \\
\mathrm{MgO} \\
\mathrm{CaO} \\
\mathrm{BaO} \\
\mathrm{Na} 20 \\
\mathrm{~K} 20\end{array}$ & $\begin{array}{c}77.2 \\
0.09 \\
12.3 \\
0.74 \\
0.00 \\
0.14 \\
0.04 \\
4.36 \\
5.07\end{array}$ & $\begin{array}{c}77.1 \\
0.10 \\
12.4 \\
0.81 \\
0.00 \\
0.19 \\
0.12 \\
4.48 \\
4.88\end{array}$ & $\begin{array}{c}\text { 0xides } \\
76.8 \\
0.10 \\
12.5 \\
0.66 \\
0.00 \\
0.21 \\
0.21 \\
4.51 \\
4.94\end{array}$ & $\begin{array}{c}\text { Recalcul } \\
77.1 \\
0.05 \\
12.1 \\
0.87 \\
0.01 \\
0.21 \\
0.21 \\
4.54 \\
4.86\end{array}$ & $\begin{array}{c}\text { ced to } \\
77.3 \\
0.03 \\
12.5 \\
0.76 \\
0.02 \\
0.47 \\
0.06 \\
3.56 \\
5.26\end{array}$ & $\begin{array}{l}\% \\
77.5 \\
0.05 \\
12.4 \\
0.80 \\
0.00 \\
0.52 \\
0.00 \\
3.49 \\
5.22\end{array}$ & $\begin{array}{c}77.4 \\
0.05 \\
12.3 \\
0.67 \\
0.00 \\
0.50 \\
0.15 \\
3.70 \\
5.21\end{array}$ & $\begin{array}{c}77.3 \\
0.07 \\
12.7 \\
0.70 \\
0.00 \\
0.51 \\
0.00 \\
3.68 \\
5.04\end{array}$ \\
\hline
\end{tabular}


APPENDIX E (cont)

Orill Hole USW H-5

\begin{tabular}{|c|c|c|c|c|c|c|}
\hline $\begin{array}{l}\text { Depth } \\
(\mathrm{ft}) \\
\text { Unit }\end{array}$ & $\begin{array}{l}1762 \\
\text { Tht }\end{array}$ & Tht & Tht & TCD & TCD & Tcp \\
\hline $\begin{array}{l}\mathrm{SiO} 2 \\
\mathrm{TiO} 2 \\
\mathrm{~A} 1203 \\
\mathrm{Fe} 203 \\
\mathrm{MgO} \\
\mathrm{CaO} \\
\mathrm{BaO} \\
\mathrm{Na2O} \\
\mathrm{K} 2 \mathrm{O}\end{array}$ & $\begin{array}{c}73.0 \\
0.02 \\
12.0 \\
0.71 \\
0.00 \\
0.45 \\
0.00 \\
3.41 \\
4.90\end{array}$ & $\begin{array}{c}74.4 \\
0.02 \\
11.8 \\
0.69 \\
0.00 \\
0.53 \\
0.23 \\
3.30 \\
5.13\end{array}$ & $\begin{array}{c}74.6 \\
0.00 \\
11.7 \\
0.47 \\
0.00 \\
0.52 \\
0.00 \\
3.48 \\
5.18\end{array}$ & $\begin{array}{c}72.7 \\
0.03 \\
11.7 \\
0.79 \\
0.00 \\
0.46 \\
0.00 \\
3.04 \\
5.02\end{array}$ & $\begin{array}{c}72.5 \\
0.04 \\
11.7 \\
0.80 \\
0.00 \\
0.39 \\
0.00 \\
2.92 \\
5.15\end{array}$ & $\begin{array}{c}72.3 \\
0.05 \\
11.8 \\
0.84 \\
0.00 \\
0.38 \\
0.00 \\
2.99 \\
5.00\end{array}$ \\
\hline Total & 94.5 & 96.1 & 95.9 & 93.7 & 93.5 & 93.4 \\
\hline $\begin{array}{l}\mathrm{Si} \\
\mathrm{Ti} \\
\mathrm{Al} \\
\mathrm{Fe} \\
\mathrm{Mg} \\
\mathrm{Ca} \\
\mathrm{Ba} \\
\mathrm{Na} \\
\mathrm{K}\end{array}$ & $\begin{array}{r}72.2 \\
0.01 \\
14.00 \\
0.53 \\
0.00 \\
0.48 \\
0.00 \\
6.54 \\
6.19\end{array}$ & $\begin{array}{c}\text { Cat } \\
72.6 \\
0.01 \\
13.58 \\
0.51 \\
0.00 \\
0.55 \\
0.09 \\
6.25 \\
6.39\end{array}$ & $\begin{array}{c}\text { Perce } \\
72.7 \\
0.00 \\
13.44 \\
0.34 \\
0.00 \\
0.54 \\
0.00 \\
6.57 \\
6.44\end{array}$ & $\begin{array}{c}72.8 \\
0.02 \\
13.81 \\
0.60 \\
0.00 \\
0.49 \\
0.00 \\
5.90 \\
6.41\end{array}$ & $\begin{array}{r}72.8 \\
0.03 \\
13.85 \\
0.60 \\
0.00 \\
0.42 \\
0.00 \\
5.69 \\
6.60\end{array}$ & $\begin{array}{r}72.7 \\
0.04 \\
13.99 \\
0.64 \\
0.00 \\
0.41 \\
0.00 \\
5.83 \\
6.41\end{array}$ \\
\hline \multicolumn{7}{|c|}{$\mathrm{Si} /(\mathrm{A} I+\mathrm{Fe})$} \\
\hline & 1.06 & $\begin{array}{c}1+\mathrm{Fe}) /( \\
1.01\end{array}$ & $\begin{array}{r}g+2 \mathrm{Ca}+2 \\
0.98\end{array}$ & $\begin{array}{r}(+N a+K) \\
1.08\end{array}$ & 1.10 & 1.12 \\
\hline $\begin{array}{l}\mathrm{K} \\
\mathrm{Na} \\
\mathrm{Ca}+\mathrm{Mg}\end{array}$ & $\begin{array}{r}46.8 \\
49.5 \\
3.6\end{array}$ & $\begin{array}{c}1 \% \text { Exch } \\
48.4 \\
47.4 \\
4.2\end{array}$ & $\begin{array}{c}\text { geable } \\
47.5 \\
48.5 \\
4.0\end{array}$ & $\begin{array}{r}\text { tions } \\
50.1 \\
46.1 \\
3.9\end{array}$ & $\begin{array}{r}51.9 \\
44.8 \\
3.3\end{array}$ & $\begin{array}{r}50.7 \\
46.1 \\
3.2\end{array}$ \\
\hline $\begin{array}{l}\mathrm{SiO2} \\
\mathrm{TiO} 2 \\
\mathrm{~A} 1203 \\
\mathrm{Fe} 203 \\
\mathrm{MgO} \\
\mathrm{CaO} \\
\mathrm{BaO} \\
\mathrm{Na} 20 \\
\mathrm{~K} 20\end{array}$ & $\begin{array}{r} \\
77.26 \\
0.02 \\
12.70 \\
0.75 \\
0.00 \\
0.48 \\
0.00 \\
3.61 \\
5.19\end{array}$ & $\begin{array}{c}\text { des } \operatorname{Rec} \\
77.42 \\
0.02 \\
12.28 \\
0.72 \\
0.00 \\
0.55 \\
0.24 \\
3.43 \\
5.34\end{array}$ & $\begin{array}{r}\text { culated } \\
77.75 \\
0.00 \\
12.19 \\
0.49 \\
0.00 \\
0.54 \\
0.00 \\
3.63 \\
5.40\end{array}$ & $\begin{array}{r}100 \% \\
77.55 \\
0.03 \\
12.48 \\
0.84 \\
0.00 \\
0.49 \\
0.00 \\
3.24 \\
5.36\end{array}$ & $\begin{array}{r}77.54 \\
0.04 \\
12.51 \\
0.86 \\
0.00 \\
0.42 \\
0.00 \\
3.12 \\
5.51\end{array}$ & $\begin{array}{l}0.00 \\
0.00 \\
0.00 \\
0.00 \\
0.00 \\
0.00 \\
0.00 \\
0.00 \\
0.00\end{array}$ \\
\hline
\end{tabular}


APPENDIX E (cont)

Drill Hole UE-25a\#1

\begin{tabular}{|c|c|c|c|c|c|c|c|c|}
\hline $\begin{array}{l}\text { Depth } \\
(\mathrm{ft})\end{array}$ & 226 & 226 & 251 & 251 & 251 & 251 & 251 & $27 T$ \\
\hline Unit ${ }^{a}$ & $\mathrm{Tpc}$ & $\mathrm{Tpc}$ & Tb & Tb & Tb & Tb & TD & Tb \\
\hline $\begin{array}{l}\mathrm{SiO} 02 \\
\mathrm{TiO} 02 \\
\mathrm{Al} 203 \\
\mathrm{Fe} 203 \\
\mathrm{MgO} \\
\mathrm{CaO} \\
\mathrm{BaO} \\
\mathrm{Na} 20 \\
\mathrm{~K} 20\end{array}$ & $\begin{array}{c}73.7 \\
0.16 \\
12.6 \\
0.77 \\
0.05 \\
0.42 \\
0.00 \\
4.05 \\
5.24\end{array}$ & $\begin{array}{c}73.9 \\
0.21 \\
12.6 \\
0.75 \\
0.02 \\
0.47 \\
0.00 \\
4.04 \\
5.43\end{array}$ & $\begin{array}{c}72.5 \\
0.14 \\
11.3 \\
0.71 \\
0.00 \\
0.47 \\
0.00 \\
2.79 \\
5.58\end{array}$ & $\begin{array}{c}72.5 \\
0.07 \\
11.5 \\
0.81 \\
0.00 \\
0.43 \\
0.00 \\
3.24 \\
5.06\end{array}$ & $\begin{array}{c}73.1 \\
0.10 \\
11.4 \\
0.67 \\
0.02 \\
0.39 \\
0.00 \\
3.19 \\
5.42\end{array}$ & $\begin{array}{c}68.4 \\
0.00 \\
14.3 \\
1.76 \\
0.18 \\
0.75 \\
0.06 \\
3.89 \\
5.72\end{array}$ & $\begin{array}{c}73.0 \\
0.08 \\
11.3 \\
0.69 \\
0.00 \\
0.38 \\
0.00 \\
3.33 \\
5.05\end{array}$ & $\begin{array}{c}70.5 \\
0.00 \\
13.4 \\
0.92 \\
0.05 \\
0.43 \\
0.07 \\
3.48 \\
5.92\end{array}$ \\
\hline lotal & 97.0 & 97.5 & 93.5 & 93.6 & 94.3 & 95.0 & 93.8 & 94.7 \\
\hline $\begin{array}{l}\mathrm{Si} \\
\mathrm{Ti} \\
\mathrm{Al} \\
\mathrm{Fe} \\
\mathrm{Mg} \\
\mathrm{Ca} \\
\mathrm{Ba} \\
\mathrm{Na} \\
\mathrm{K}\end{array}$ & $\begin{array}{c}70.6 \\
0.12 \\
14.3 \\
0.56 \\
0.07 \\
0.43 \\
0.00 \\
7.53 \\
6.41\end{array}$ & $\begin{array}{c}70.5 \\
0.15 \\
14.2 \\
0.54 \\
0.03 \\
0.48 \\
0.00 \\
7.47 \\
6.61\end{array}$ & $\begin{array}{c}72.9 \\
0.11 \\
13.4 \\
0.54 \\
0.00 \\
0.51 \\
0.00 \\
5.44 \\
7.15\end{array}$ & $\begin{array}{c}\text { ion Per } \\
72.5 \\
0.05 \\
13.6 \\
0.61 \\
0.00 \\
0.46 \\
0.00 \\
6.29 \\
6.46\end{array}$ & $\begin{array}{c}n \mathrm{t} \\
72.6 \\
0.07 \\
13.3 \\
0.50 \\
0.03 \\
0.42 \\
0.00 \\
6.15 \\
6.87\end{array}$ & $\begin{array}{c}66.7 \\
0.00 \\
16.5 \\
1.29 \\
0.26 \\
0.78 \\
0.02 \\
7.36 \\
7.12\end{array}$ & $\begin{array}{c}72.9 \\
0.06 \\
13.3 \\
0.52 \\
0.00 \\
0.41 \\
0.00 \\
6.45 \\
6.43\end{array}$ & $\begin{array}{c}69.2 \\
0.00 \\
15.5 \\
0.68 \\
0.07 \\
0.45 \\
0.03 \\
6.63 \\
7.42\end{array}$ \\
\hline \multicolumn{9}{|c|}{$\mathrm{Si} /(\mathrm{A})+\mathrm{Fe})$} \\
\hline & 0.99 & 0.98 & $\begin{array}{c}(A 1+F e) / \\
1.02\end{array}$ & $\begin{array}{r}\mathrm{Mg}+2 \mathrm{Ca}+ \\
1.04\end{array}$ & $\begin{array}{c}3 a+N a+K) \\
0.99\end{array}$ & 1.07 & 1.01 & 1.07 \\
\hline $\begin{array}{l}\mathrm{K} \\
\mathrm{Na} \\
\mathrm{Ca}+\mathrm{Mg}\end{array}$ & $\begin{array}{r}44.4 \\
52.1 \\
3.5\end{array}$ & $\begin{array}{r}45.3 \\
51.2 \\
3.5\end{array}$ & $\begin{array}{c}\text { Mo1\% Exc } \\
54.6 \\
41.5 \\
3.9\end{array}$ & $\begin{array}{c}\text { ngeable } \\
48.9 \\
47.6 \\
3.5\end{array}$ & $\begin{array}{c}\text { ations } \\
51.0 \\
45.7 \\
3.3\end{array}$ & $\begin{array}{r}45.9 \\
47.4 \\
6.7\end{array}$ & $\begin{array}{r}48.4 \\
48.5 \\
3.1\end{array}$ & $\begin{array}{r}50.9 \\
45.5 \\
3.6\end{array}$ \\
\hline $\begin{array}{l}\mathrm{SiO} 02 \\
\mathrm{Ti02} \\
\mathrm{A} 1203 \\
\mathrm{Fe} 203 \\
\mathrm{MgO} \\
\mathrm{CaO} \\
\mathrm{Ba0} \\
\mathrm{Na} 20 \\
\mathrm{~K} 20\end{array}$ & $\begin{array}{c}76.0 \\
0.16 \\
13.0 \\
0.79 \\
0.05 \\
0.43 \\
0.00 \\
4.18 \\
5.40\end{array}$ & $\begin{array}{c}75.8 \\
0.22 \\
13.0 \\
0.77 \\
0.02 \\
0.48 \\
0.00 \\
4.14 \\
5.57\end{array}$ & $\begin{array}{c}\text { Oxides } \operatorname{Re} \\
77.5 \\
0.15 \\
12.1 \\
0.76 \\
0.00 \\
0.50 \\
0.00 \\
2.98 \\
5.97\end{array}$ & $\begin{array}{c}\text { lculate } \\
77.4 \\
0.07 \\
12.3 \\
0.87 \\
0.00 \\
0.46 \\
0.00 \\
3.46 \\
5.41\end{array}$ & $\begin{array}{c}\text { to } 100 \% \\
77.5 \\
0.11 \\
12.1 \\
0.71 \\
0.02 \\
0.41 \\
0.00 \\
3.38 \\
5.75\end{array}$ & $\begin{array}{c}71.9 \\
0.00 \\
15.1 \\
1.85 \\
0.19 \\
0.79 \\
0.06 \\
4.09 \\
6.02\end{array}$ & $\begin{array}{c}77.8 \\
0.09 \\
12.0 \\
0.74 \\
0.00 \\
0.41 \\
0.00 \\
3.55 \\
5.38\end{array}$ & $\begin{array}{c}74.4 \\
0.00 \\
14.1 \\
0.97 \\
0.05 \\
0.45 \\
0.07 \\
3.67 \\
6.25\end{array}$ \\
\hline
\end{tabular}


APPENDIX E (cont)

Drill Hole UE-25a\#1

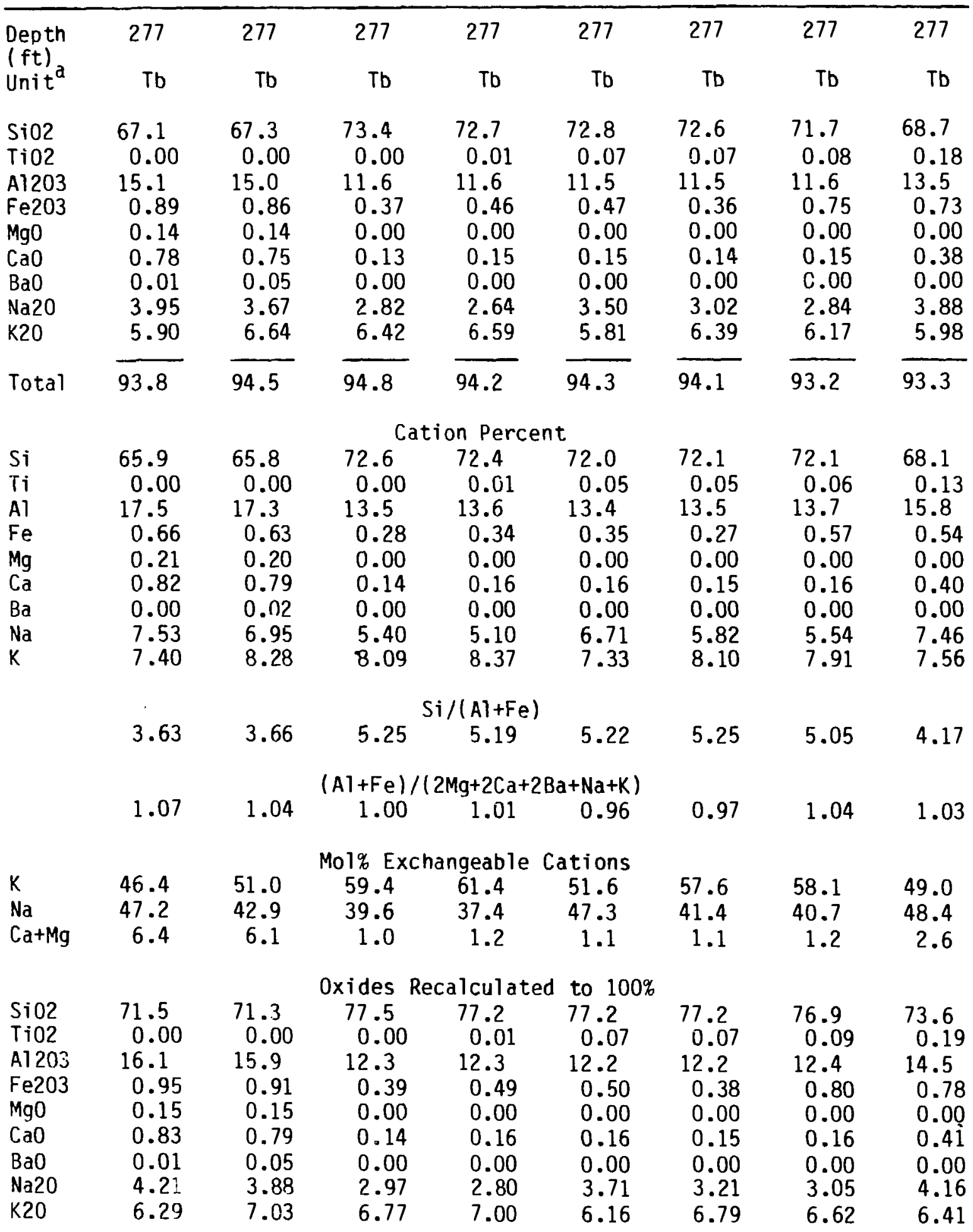


APPENOIX E (cont)

Drill Hole UE-25a\#1

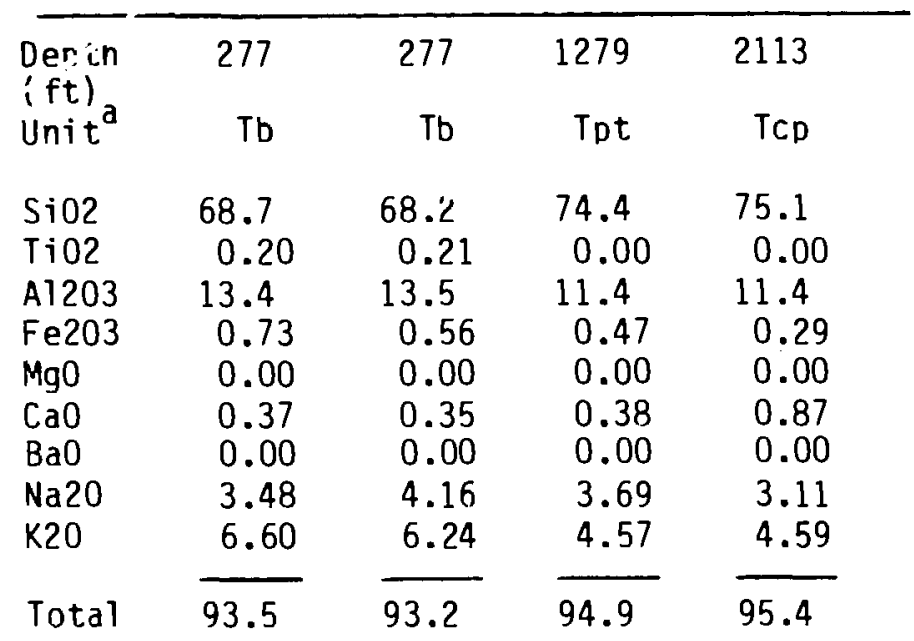

Cation Percent

\begin{tabular}{|c|c|c|c|c|}
\hline $\mathrm{Si}$ & 68.2 & 67.4 & 73.2 & 73.9 \\
\hline $\mathrm{Ti}$ & 0.15 & 0.16 & 0.00 & 0.00 \\
\hline Al & 15.7 & 15.8 & 13.3 & 13.2 \\
\hline $\mathrm{Fe}$ & 0.54 & 0.42 & 0.35 & 0.21 \\
\hline $\mathrm{Mg}$ & 0.00 & 0.00 & 0.00 & 0.00 \\
\hline $\mathrm{Ca}$ & 0.39 & 0.37 & 0.40 & 0.92 \\
\hline $\mathrm{Ba}$ & 0.00 & 0.00 & 0.00 & 0.00 \\
\hline $\mathrm{Na}$ & 6.69 & 7.98 & 7.04 & 5.93 \\
\hline $\mathrm{K}$ & 8.35 & 7.88 & 5.74 & 5.76 \\
\hline \multicolumn{5}{|c|}{$\mathrm{Si} /(\mathrm{Al}+\mathrm{Fe})$} \\
\hline & 4.19 & 4.17 & 5.38 & 5.50 \\
\hline \multicolumn{5}{|c|}{$(\mathrm{Al}+\mathrm{Fe}) /(2 \mathrm{Mg}+2 \mathrm{Ca}+2 \mathrm{Ba}+\mathrm{Na}+\mathrm{K})$} \\
\hline & 1.03 & 0.98 & 1.00 & 0.99 \\
\hline \multicolumn{5}{|c|}{ Mol\% Exchangeable Cations } \\
\hline K & 54.1 & 48.5 & 43.5 & 45.7 \\
\hline $\mathrm{Na}$ & 43.4 & 49.2 & 53.4 & 47.0 \\
\hline $\mathrm{Ca}+\mathrm{Mg}$ & 2.5 & 2.3 & 3.0 & 7.3 \\
\hline \multicolumn{5}{|c|}{ 0xides Recalculated to $100 \%$} \\
\hline Si02 & 73.5 & 73.1 & 78.4 & 78.8 \\
\hline $\mathrm{TiO}$ & 0.21 & 0.23 & 0.00 & 0.00 \\
\hline A1203 & 14.4 & 14.5 & 12.0 & 11.9 \\
\hline $\mathrm{Fe} 203$ & 0.78 & 0.60 & 0.50 & 0.30 \\
\hline Mq0 & 0.00 & 0.00 & 0.00 & 0.00 \\
\hline $\mathrm{CaO}$ & 0.40 & 0.38 & 0.40 & 0.91 \\
\hline Ba0 & 0.00 & 0.00 & 0.00 & 0.00 \\
\hline $\mathrm{Na20}$ & 3.72 & 4.46 & 3.89 & 3.26 \\
\hline K20 & 7.06 & 6.69 & 4.81 & 4.81 \\
\hline
\end{tabular}


APPENDIX E (cont)

Drill Hole J-13

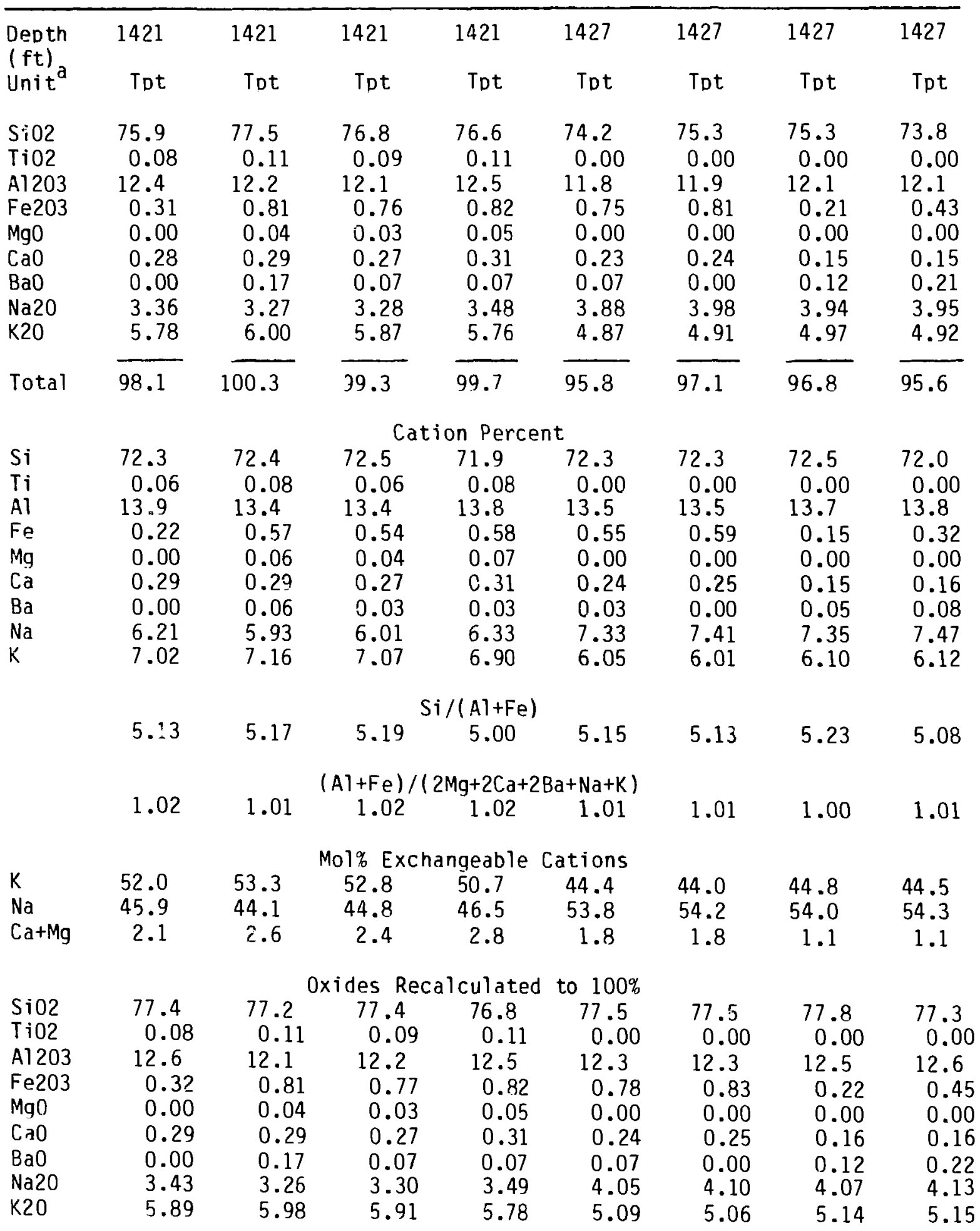


APPENDIX E (cont)

Drill Hole J-13

\begin{tabular}{|c|c|c|c|c|c|}
\hline $\begin{array}{l}\text { Depth } \\
(f t) \\
\text { Unit }\end{array}$ & Tht & 1507 & 1507 & Tht & Tht \\
\hline $\begin{array}{l}\mathrm{SiO} 2 \\
\mathrm{TiO} 2 \\
\mathrm{~A} 1203 \\
\mathrm{Fe} 203 \\
\mathrm{MgO} \\
\mathrm{CaO} \\
\mathrm{BaO} \\
\mathrm{Na} 20 \\
\mathrm{~K} 20\end{array}$ & $\begin{array}{c}74.2 \\
0.00 \\
12.0 \\
0.73 \\
0.00 \\
0.40 \\
0.00 \\
3.33 \\
5.78\end{array}$ & $\begin{array}{c}74.4 \\
0.00 \\
12.1 \\
0.73 \\
0.00 \\
0.42 \\
0.02 \\
3.37 \\
5.73\end{array}$ & $\begin{array}{c}74.9 \\
0.00 \\
12.0 \\
0.83 \\
0.00 \\
0.35 \\
0.00 \\
3.36 \\
5.86\end{array}$ & $\begin{array}{c}74.3 \\
0.08 \\
11.9 \\
0.58 \\
0.04 \\
0.46 \\
0.09 \\
3.21 \\
4.98\end{array}$ & $\begin{array}{c}72.3 \\
0.08 \\
11.6 \\
0.74 \\
0.05 \\
0.45 \\
0.00 \\
3.25 \\
5.01\end{array}$ \\
\hline Total & 96.5 & 96.7 & 97.3 & 95.7 & 93.5 \\
\hline $\begin{array}{l}\mathrm{Si} \\
\mathrm{Ti} \\
\mathrm{Al} \\
\mathrm{Fe} \\
\mathrm{Mg} \\
\mathrm{Ca} \\
\mathrm{Ba} \\
\mathrm{Na} \\
\mathrm{K}\end{array}$ & $\begin{array}{c}71.9 \\
0.00 \\
13.7 \\
0.53 \\
0.00 \\
0.42 \\
0.00 \\
6.26 \\
7.15\end{array}$ & $\begin{array}{c}\text { Cation } \\
71.9 \\
0.00 \\
13.8 \\
0.53 \\
0.00 \\
0.44 \\
0.01 \\
6.32 \\
7.07\end{array}$ & $\begin{array}{c}\text { cent } \\
72.0 \\
0.00 \\
13.6 \\
0.60 \\
0.00 \\
0.36 \\
0.00 \\
6.26 \\
7.18\end{array}$ & $\begin{array}{c}72.9 \\
0.06 \\
13.8 \\
0.43 \\
0.06 \\
0.48 \\
0.03 \\
6.10 \\
6.23\end{array}$ & $\begin{array}{c}72.4 \\
0.06 \\
13.7 \\
0.56 \\
0.07 \\
0.48 \\
0.00 \\
6.31 \\
6.40\end{array}$ \\
\hline & $\begin{array}{r}5.05 \\
(\mathrm{~A})+ \\
1.00\end{array}$ & $\begin{array}{r}\mathrm{Si} / 1 \\
5.03 \\
\\
/ 12 \mathrm{Mg} \\
1.00\end{array}$ & $\begin{array}{r}\text { Fe) } \\
5.06 \\
a+2 B a+ \\
1.00\end{array}$ & $\begin{array}{r}5.13 \\
+K) \quad 1.05\end{array}$ & 5.07 \\
\hline $\begin{array}{l}\mathrm{K} \\
\mathrm{Na} \\
\mathrm{Ca}+\mathrm{Mg}\end{array}$ & $\begin{array}{c}\text { Mol } \\
51.7 \\
45.3 \\
3.0\end{array}$ & $\begin{array}{c}\text { Exchan } \\
51.1 \\
45.7 \\
3.1\end{array}$ & $\begin{array}{c}\text { ble Ca } \\
52.0 \\
45.3 \\
2.6\end{array}$ & $\begin{array}{l}\text { ons } \\
48.4 \\
47.4 \\
4.2\end{array}$ & $\begin{array}{r}48.2 \\
47.6 \\
4.2\end{array}$ \\
\hline $\begin{array}{l}\mathrm{SiO} 2 \\
\mathrm{TiO} 2 \\
\mathrm{~A} 1203 \\
\mathrm{Fe} 203 \\
\mathrm{MgO} \\
\mathrm{CaO} \\
\mathrm{BaO} \\
\mathrm{Na} 20 \\
\mathrm{~K} 20\end{array}$ & $\begin{array}{c}0 \times i c \\
76.9 \\
0.00 \\
12.4 \\
0.76 \\
0.00 \\
0.41 \\
0.00 \\
3.45 \\
5.99\end{array}$ & $\begin{array}{c}\text { Recal } \\
76.9 \\
0.00 \\
12.5 \\
0.75 \\
0.00 \\
0.43 \\
0.02 \\
3.49 \\
5.93\end{array}$ & $\begin{array}{c}\text { ated } t \\
76.9 \\
0.00 \\
12.4 \\
0.85 \\
0.00 \\
0.36 \\
0.00 \\
3.45 \\
6.02\end{array}$ & $\begin{array}{c}100 \% \\
77.7 \\
0.08 \\
12.4 \\
0.61 \\
0.04 \\
0.48 \\
0.09 \\
3.35 \\
5.20\end{array}$ & $\begin{array}{c}77.3 \\
0.09 \\
12.4 \\
0.79 \\
0.05 \\
0.48 \\
0.00 \\
3.48 \\
5.36\end{array}$ \\
\hline
\end{tabular}


Outcrop at Prow Pass, Northern Yucca Mountain.

Sample Number: 3-15-82-11; Nevada State Coordinates ( $\mathrm{ft}$ ) 786550N, 551400E

Partially welded tuff near base of Topopah Spring Member, Paintbrush Tuff

\begin{tabular}{|c|c|c|c|c|c|c|c|c|}
\hline $\begin{array}{l}\mathrm{SiO} 2 \\
\mathrm{TiO} 2 \\
\mathrm{Al} 203 \\
\mathrm{Fe} 203 \\
\mathrm{HgO} \\
\mathrm{CaO} \\
\mathrm{BaC} \\
\mathrm{Na} 20 \\
\mathrm{~K} 2 \mathrm{O}\end{array}$ & $\begin{array}{c}72.4 \\
0.02 \\
11.6 \\
0.54 \\
0.00 \\
0.40 \\
0.00 \\
3.52 \\
4.73\end{array}$ & $\begin{array}{c}72.7 \\
0.05 \\
.1 .6 \\
0.46 \\
0.00 \\
0.41 \\
0.00 \\
3.53 \\
4.77\end{array}$ & $\begin{array}{c}74.6 \\
0.05 \\
11.5 \\
0.35 \\
0.00 \\
0.43 \\
0.00 \\
3.46 \\
4.44\end{array}$ & $\begin{array}{c}73.8 \\
0.03 \\
11.8 \\
0.44 \\
0.00 \\
0.45 \\
0.00 \\
3.58 \\
4.58\end{array}$ & $\begin{array}{c}72.6 \\
0.03 \\
11.5 \\
0.47 \\
0.00 \\
0.40 \\
0.00 \\
3.33 \\
4.70\end{array}$ & $\begin{array}{c}72.7 \\
0.00 \\
11.6 \\
0.66 \\
0.00 \\
0.45 \\
0.00 \\
3.54 \\
4.79\end{array}$ & $\begin{array}{c}72.7 \\
0.00 \\
-1.7 \\
0.52 \\
0.01 \\
0.42 \\
0.00 \\
3.68 \\
4.75\end{array}$ & $\begin{array}{c}72.3 \\
0.03 \\
11.7 \\
0.61 \\
0.00 \\
0.41 \\
0.00 \\
3.52 \\
4.82\end{array}$ \\
\hline Total & 93.1 & 93.5 & 94.9 & 94.6 & 93.0 & 93.7 & 93.8 & 93.3 \\
\hline $\begin{array}{l}\mathrm{Si} \\
\mathrm{Ti} \\
\mathrm{Al} \\
\mathrm{Fe} \\
\mathrm{Mg} \\
\mathrm{Ca} \\
\mathrm{Ea} \\
\mathrm{Na} \\
\mathrm{K}\end{array}$ & $\begin{array}{c}72.6 \\
0.02 \\
13.7 \\
0.41 \\
0.00 \\
0.43 \\
0.00 \\
6.85 \\
6.05\end{array}$ & $\begin{array}{c}72.6 \\
0.04 \\
13.5 \\
0.35 \\
0.00 \\
0.44 \\
0.00 \\
6.84 \\
6.08\end{array}$ & $\begin{array}{c}{ }^{C a} \\
73.6 \\
0.04 \\
13.4 \\
0.26 \\
0.00 \\
0.45 \\
0.00 \\
6.62 \\
5.59\end{array}$ & $\begin{array}{c}\text { on Perc } \\
72.9 \\
0.02 \\
13.7 \\
0.33 \\
0.00 \\
0.48 \\
0.00 \\
6.85 \\
5.77\end{array}$ & $\begin{array}{c}\mathrm{t} \\
73.1 \\
0.02 \\
13.6 \\
0.36 \\
0.00 \\
0.43 \\
0.00 \\
6.49 \\
6.03\end{array}$ & $\begin{array}{c}72.5 \\
0.00 \\
13.6 \\
0.50 \\
0.00 \\
0.48 \\
0.00 \\
6.85 \\
6.10\end{array}$ & $\begin{array}{c}72.3 \\
0.00 \\
13.7 \\
0.39 \\
0.01 \\
0.45 \\
0.00 \\
7.10 \\
6.03\end{array}$ & $\begin{array}{c}72.3 \\
0.02 \\
13.8 \\
0.46 \\
0.00 \\
0.44 \\
0.00 \\
6.83 \\
6.15\end{array}$ \\
\hline \multicolumn{9}{|c|}{$\mathrm{Si} /(\mathrm{Al}+\mathrm{Fe})$} \\
\hline & 1.02 & 1.01 & $\begin{array}{c}A 1+F e) / \\
1.04\end{array}$ & $\begin{array}{r}\mathrm{Mg}+2 \mathrm{Ca} \\
1.03\end{array}$ & $\begin{array}{c}a+N a+K) \\
1.04\end{array}$ & 1.01 & 1.00 & 1.03 \\
\hline $\begin{array}{l}\mathrm{K} \\
\mathrm{Na} \\
\mathrm{Ca}+\mathrm{Mg}\end{array}$ & $\begin{array}{r}45.4 \\
51.4 \\
3.2\end{array}$ & $\begin{array}{r}45.5 \\
51.2 \\
3.3\end{array}$ & $\begin{array}{c}01 \% \text { Exo } \\
44.1 \\
52.3 \\
3.6\end{array}$ & $\begin{array}{c}\text { ngeable } \\
44.0 \\
52.3 \\
3.6\end{array}$ & $\begin{array}{c}\text { ations } \\
46.5 \\
50.1 \\
3.3\end{array}$ & $\begin{array}{r}45.4 \\
51.0 \\
3.6\end{array}$ & $\begin{array}{r}44.4 \\
52.2 \\
3.4\end{array}$ & $\begin{array}{r}45.8 \\
50.9 \\
3.3\end{array}$ \\
\hline $\begin{array}{l}\mathrm{SiO} 2 \\
\mathrm{TiO} 2 \\
\mathrm{~A} 1203 \\
\mathrm{Fe} 203 \\
\mathrm{MgO} \\
\mathrm{CaO} \\
\mathrm{BaO} \\
\mathrm{Na} 20 \\
\mathrm{~K} 20\end{array}$ & $\begin{array}{c}77.7 \\
0.02 \\
12.4 \\
0.58 \\
0.00 \\
0.43 \\
0.00 \\
3.78 \\
5.08\end{array}$ & $\begin{array}{c}77.8 \\
0.05 \\
12.3 \\
0.49 \\
0.00 \\
0.44 \\
0.00 \\
3.78 \\
5.10\end{array}$ & $\begin{array}{c}\text { ides } \operatorname{Re} \\
78.7 \\
0.05 \\
12.1 \\
0.37 \\
0.00 \\
0.45 \\
0.00 \\
3.65 \\
4.68\end{array}$ & $\begin{array}{c}\text { lculat } \\
78.0 \\
0.03 \\
12.4 \\
0.46 \\
0.00 \\
0.48 \\
0.00 \\
3.78 \\
4.84\end{array}$ & $\begin{array}{c}\text { to } 100 \% \\
78.1 \\
0.03 \\
12.3 \\
0.51 \\
0.00 \\
0.43 \\
0.00 \\
3.58 \\
5.05\end{array}$ & $\begin{array}{c}77.6 \\
0.00 \\
12.3 \\
0.70 \\
0.00 \\
0.48 \\
0.00 \\
3.78 \\
5.11\end{array}$ & $\begin{array}{c}77.5 \\
0.00 \\
12.5 \\
0.55 \\
0.01 \\
0.45 \\
0.00 \\
3.92 \\
5.06\end{array}$ & $\begin{array}{c}77.4 \\
0.03 \\
12.5 \\
0.65 \\
0.00 \\
0.44 \\
0.00 \\
3.77 \\
5.16\end{array}$ \\
\hline
\end{tabular}




\section{APPENDIX E (cont)}

Outcrop at Prow Pass, Northerr Yucca Mounta in

Sample Number: $3-15-82-11$

Partially welcien tuff near base of Topopah Spring Member, Haintbrush Tuff

$\begin{array}{lccc}\text { Si02 } & 70.9 & 72.3 & 71.6 \\ \text { Ti02 } & 0.04 & 0.04 & 0.00 \\ \text { A1203 } & 11.8 & 11.9 & 11.6 \\ \mathrm{Fe} 203 & 0.69 & 0.35 & 0.63 \\ \text { Mg0 } & 0.00 & 0.00 & 0.00 \\ \mathrm{Ca0} & 0.43 & 0.39 & 0.48 \\ \mathrm{Ba0} & 0.00 & 0.00 & 0.00 \\ \mathrm{Na20} & 3.38 & 3.48 & 3.31 \\ \text { K20 } & 4.74 & \frac{4.72}{93.2} & \frac{4.72}{92.3} \\ \text { Total } & \frac{42.0}{92} & \end{array}$

Cation Percent

$\begin{array}{lccc}\mathrm{Si} & 72.1 & 72.4 & 72.5 \\ \mathrm{Ti} & 0.03 & 0.03 & 0.00 \\ \mathrm{Al} & 14.1 & 14.1 & 13.9 \\ \mathrm{Fe} & 0.53 & 0.26 & 0.48 \\ \mathrm{Mg} & 0.00 & 0.00 & 0.00 \\ \mathrm{Ca} & 0.47 & 0.42 & 0.52 \\ \mathrm{Ba} & 0.00 & 0.00 & 0.00 \\ \mathrm{Na} & 6.66 & 6.76 & 6.50 \\ \mathrm{~K} & 6.14 & 6.03 & 6.10\end{array}$

$\mathrm{Si} /(\mathrm{Al}+\mathrm{Fe})$
4.92
5.04
5.04

$(\mathrm{Al}+\mathrm{Fe}) /(2 \mathrm{Mg}+2 \mathrm{Ca}+2 \mathrm{Ba}+\mathrm{Na}+\mathrm{K})$ $\begin{array}{lll}1.07 & 1.05 \quad 1.06\end{array}$

Mo1\% Exchangeable Cations

$\begin{array}{lrrr}\mathrm{K} & 46.3 & 45.7 & 46.5 \\ \mathrm{Na} & 50.2 & 51.2 & 49.5 \\ \mathrm{Ca}+\mathrm{Mg} & 3.5 & 3.2 & 4.0\end{array}$

\begin{tabular}{lccc}
\multicolumn{1}{c}{ Oxides } & Recalculated to & $100 \%$ \\
Si02 & 77.1 & 77.5 & 77.5 \\
Ti02 & 0.04 & 0.04 & 0.00 \\
A1203 & 12.8 & 12.8 & 12.6 \\
Fe203 & 0.75 & 0.38 & 0.68 \\
Mg0 & 0.00 & 0.00 & 0.00 \\
Ca0 & 0.47 & 0.42 & 0.52 \\
Ba0 & 0.00 & 0.00 & 0.00 \\
Na20 & 3.67 & 3.74 & 3.58 \\
K20 & 5.15 & 5.07 & 5.11
\end{tabular}




\section{APPENDIX $F$}

CLINOPTILOLITE COMPOSITIONS DETERMINED BY ELECTRON MICROPROBE, YUCCA MOUNTAIN, NEVADA

\begin{tabular}{|c|c|c|c|c|c|c|c|c|}
\hline \multirow{3}{*}{$\begin{array}{l}\text { Depth } \\
(\mathrm{ft}) \\
\text { Unit }\end{array}$} & \multicolumn{8}{|c|}{ Drill Hole USW G-1 } \\
\hline & 1286 & 1436 & 1561 & 1561 & 1561 & 1639 & 1774 & 1774 \\
\hline & Tot & Tht & Tht & Tht & Tht & Tht & Tht & Tht \\
\hline $\begin{array}{l}\mathrm{i02} \\
\mathrm{TiO} 02 \\
\mathrm{Al} 203 \\
\mathrm{Fe} 203 \\
\mathrm{MgO} \\
\mathrm{CaO} \\
\mathrm{BaO} \\
\mathrm{Na20} \\
\mathrm{K} 20\end{array}$ & $\begin{array}{l}63.5 \\
.0 .00 \\
12.6 \\
0.00 \\
0.28 \\
5.09 \\
0.50 \\
0.87 \\
0.57\end{array}$ & $\begin{array}{c}68.9 \\
0.00 \\
12.4 \\
0.00 \\
0.00 \\
0.88 \\
0.00 \\
2.29 \\
5.89\end{array}$ & $\begin{array}{c}65.7 \\
0.00 \\
11.6 \\
0.02 \\
0.00 \\
0.62 \\
0.00 \\
2.67 \\
4.87\end{array}$ & $\begin{array}{c}69.5 \\
0.00 \\
11.8 \\
0.18 \\
0.00 \\
0.56 \\
0.00 \\
2.30 \\
5.64\end{array}$ & $\begin{array}{c}63.4 \\
0.00 \\
10.9 \\
0.00 \\
0.00 \\
0.57 \\
0.00 \\
2.29 \\
4.99\end{array}$ & $\begin{array}{c}66.5 \\
0.00 \\
11.5 \\
0.00 \\
0.02 \\
0.73 \\
0.09 \\
2.59 \\
4.56\end{array}$ & $\begin{array}{c}68.1 \\
0.00 \\
12.2 \\
0.00 \\
0.09 \\
1.11 \\
0.03 \\
2.84 \\
4.20\end{array}$ & $\begin{array}{c}63.8 \\
0.00 \\
11.9 \\
0.00 \\
0.12 \\
1.43 \\
0.12 \\
3.18 \\
2.25\end{array}$ \\
\hline Total & 32.9 & 90.4 & 85.4 & 90.1 & 82.1 & 86.1 & 88.5 & 82.8 \\
\hline $\begin{array}{l}\mathrm{Si} \\
\mathrm{Ti} \\
\mathrm{Al} \\
\mathrm{Fe} \\
\mathrm{Mg} \\
\mathrm{Ca} \\
\mathrm{Ba} \\
\mathrm{Na} \\
\mathrm{K}\end{array}$ & $\begin{array}{l}29.2 \\
0.00 \\
6.86 \\
0.00 \\
0.19 \\
2.51 \\
0.00 \\
0.78 \\
0.33\end{array}$ & $\begin{array}{l}\text { Unit- } \\
29.8 \\
0.00 \\
6.32 \\
0.00 \\
0.00 \\
0.41 \\
0.00 \\
1.92 \\
3.25\end{array}$ & $\begin{array}{c}\text { e11 Comp } \\
29.9 \\
0.00 \\
6.22 \\
0.01 \\
0.00 \\
0.30 \\
0.00 \\
2.29 \\
2.83\end{array}$ & $\begin{array}{c}\text { ition } B \\
30.0 \\
0.00 \\
6.03 \\
0.06 \\
0.00 \\
0.26 \\
0.00 \\
1.93 \\
3.11\end{array}$ & $\begin{array}{c}\text { sed on } 7 \\
30.0 \\
0.00 \\
6.09 \\
0.00 \\
0.00 \\
0.29 \\
0.00 \\
2.10 \\
3.01\end{array}$ & $\begin{array}{l}(0) \\
30.0 \\
0.00 \\
6.13 \\
0.00 \\
0.01 \\
0.35 \\
0.02 \\
2.26 \\
2.62\end{array}$ & $\begin{array}{l}29.8 \\
0.00 \\
6.28 \\
0.00 \\
0.06 \\
0.52 \\
0.01 \\
2.41 \\
2.35\end{array}$ & $\begin{array}{l}29.7 \\
0.00 \\
6.50 \\
0.00 \\
0.08 \\
0.71 \\
0.02 \\
2.87 \\
1.33\end{array}$ \\
\hline & 4.26 & 4.71 & $4.80^{\circ}$ & $\begin{array}{c}(A 1+F e) \\
4.93\end{array}$ & 4.93 & 4.89 & 4.74 & 4.57 \\
\hline & 1.05 & 1.06 & $\begin{array}{c}(\mathrm{A} 1+\mathrm{Fe}) \\
1.09\end{array}$ & $\begin{array}{c}\mathrm{Mg}+2 \mathrm{Ca}+ \\
1.10\end{array}$ & $\begin{array}{r}\mathrm{Ba}+\mathrm{Na}+\mathrm{K}) \\
1.07\end{array}$ & 1.09 & 1.06 & 1.11 \\
\hline $\begin{array}{l}\mathrm{K} \\
\mathrm{Na} \\
\mathrm{Ca}+\mathrm{Mg}\end{array}$ & $\begin{array}{r}8.8 \\
20.4 \\
70.9\end{array}$ & $\begin{array}{r}58.3 \\
34.4 \\
7.3\end{array}$ & $\begin{array}{c}\text { Mol\% Exc } \\
52.1 \\
42.3 \\
5.6\end{array}$ & $\begin{array}{c}\text { ngeable } \\
58.7 \\
36.4 \\
4.9\end{array}$ & $\begin{array}{c}\text { Cations } \\
55.8 \\
38.9 \\
5.3\end{array}$ & $\begin{array}{r}49.9 \\
43.1 \\
7.0\end{array}$ & $\begin{array}{l}44.0 \\
45.2 \\
10.9\end{array}$ & $\begin{array}{l}26.7 \\
57.4 \\
15.9\end{array}$ \\
\hline
\end{tabular}

a Unit symbols defined in Table I. 


\section{APPENEIX F (cont)}

Drill Hole USW G-1

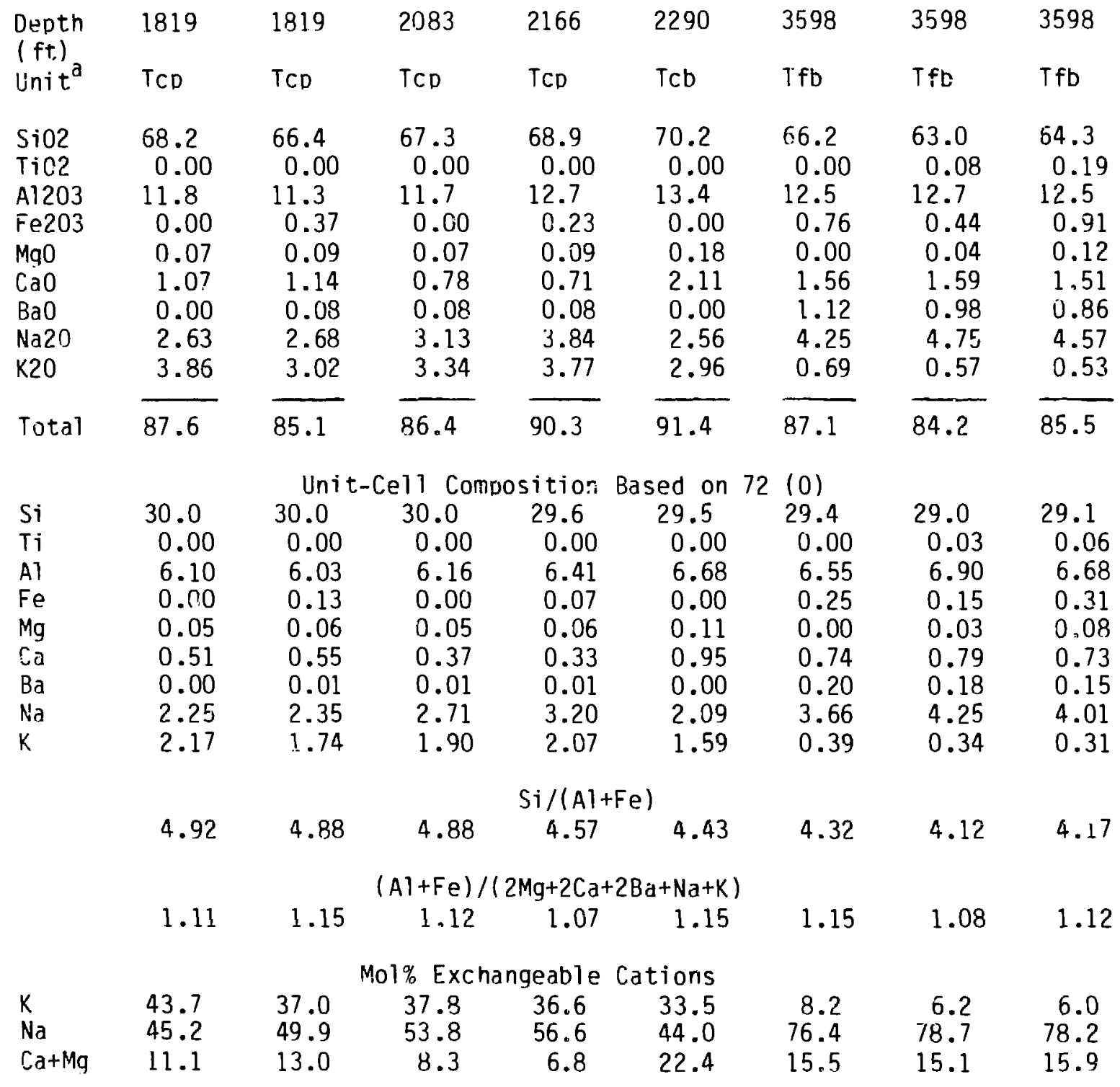




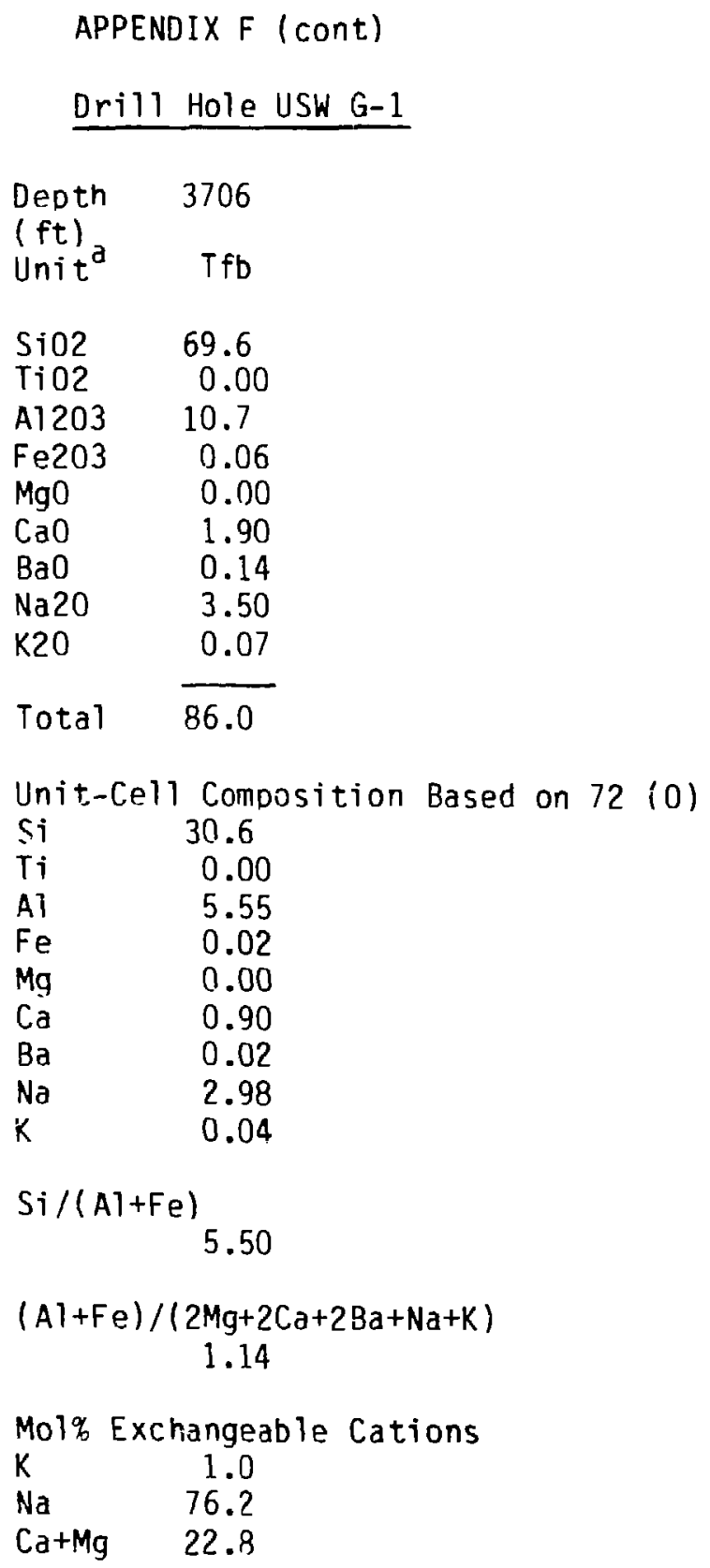


Dri11 Hole USW G-2

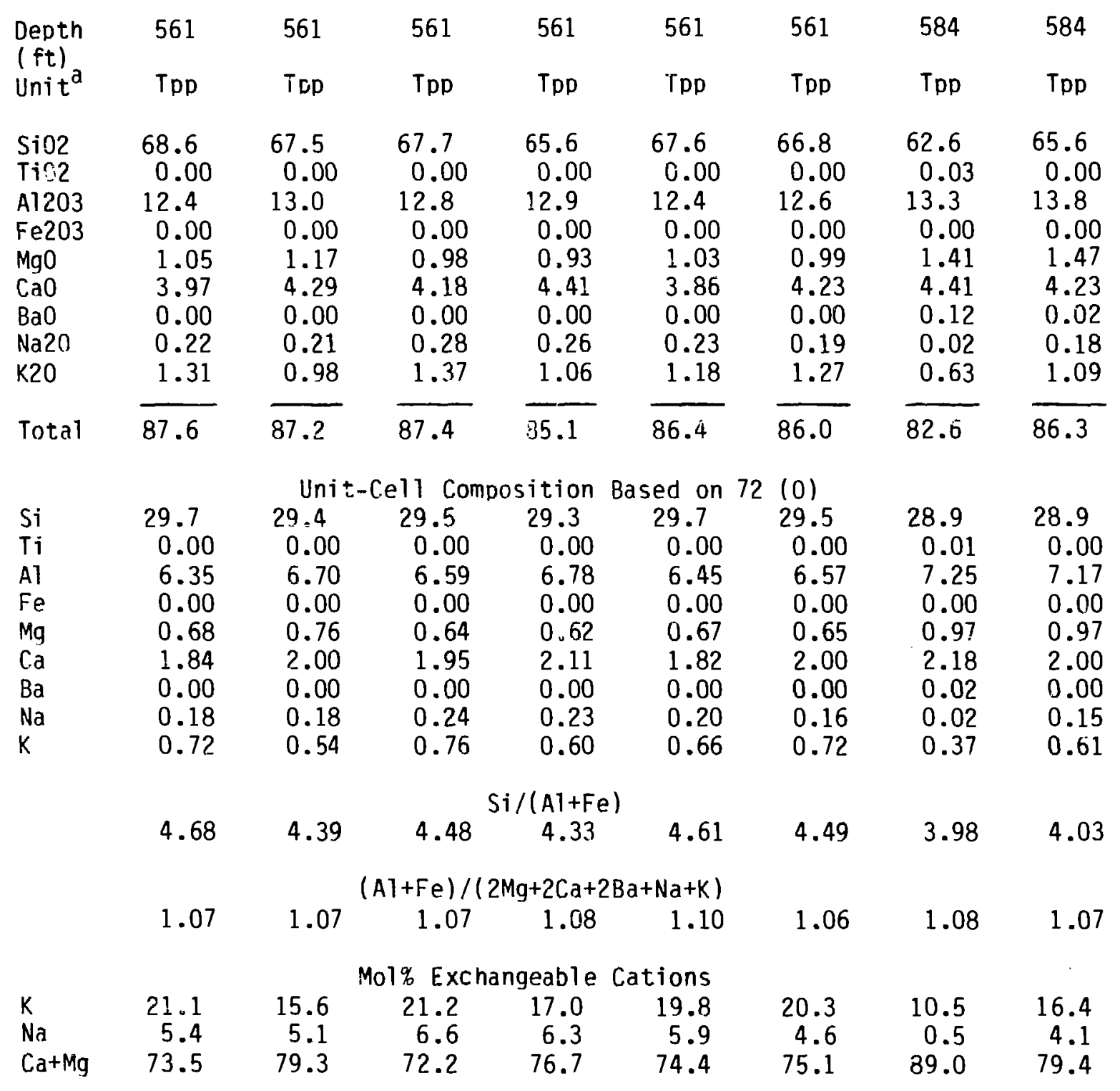




\section{APPENDIX $F$ (cont)}

\section{Orill Hole USW G-2}

\begin{tabular}{|c|c|c|c|c|c|c|c|c|}
\hline $\begin{array}{l}\text { Depth } \\
(\mathrm{ft}) \\
\text { Unit }\end{array}$ & $\begin{array}{l}581 \\
T p D\end{array}$ & $\begin{array}{l}584 \\
T p D\end{array}$ & $\begin{array}{l}584 \\
T p p\end{array}$ & $T p D$ & $T p D$ & TpD & $T D p$ & 657 \\
\hline $\begin{array}{l}\mathrm{SiO} 2 \\
\mathrm{i} i 02 \\
\mathrm{~A} ? 203 \\
\mathrm{Fe} 203 \\
\mathrm{MgO} \\
\mathrm{CaO} \\
\mathrm{BaO} \\
\mathrm{Na2O} \\
\mathrm{K} 2 \mathrm{O}\end{array}$ & $\begin{array}{c}67.0 \\
0.07 \\
13.6 \\
0.00 \\
1.35 \\
4.16 \\
0.00 \\
0.03 \\
0.75\end{array}$ & $\begin{array}{c}62.1 \\
0.00 \\
13.6 \\
0.00 \\
1.53 \\
4.03 \\
0.00 \\
0.13 \\
0.97\end{array}$ & $\begin{array}{c}61.1 \\
0.00 \\
13.5 \\
0.00 \\
1.55 \\
3.98 \\
0.07 \\
0.14 \\
0.95\end{array}$ & $\begin{array}{c}61.6 \\
0.00 \\
14.0 \\
0.00 \\
1.75 \\
4.09 \\
0.00 \\
0.12 \\
0.59\end{array}$ & $\begin{array}{c}62.9 \\
0.00 \\
14.0 \\
0.00 \\
1.86 \\
4.08 \\
0.09 \\
0.10 \\
0.54\end{array}$ & $\begin{array}{c}67.5 \\
0.00 \\
13.3 \\
0.03 \\
0.98 \\
4.11 \\
0.51 \\
0.11 \\
1.28\end{array}$ & $\begin{array}{c}67.2 \\
0.00 \\
13.4 \\
0.33 \\
0.95 \\
4.07 \\
0.42 \\
0.25 \\
1.62\end{array}$ & $\begin{array}{c}65.4 \\
0.08 \\
13.3 \\
0.26 \\
0.97 \\
3.95 \\
0.22 \\
0.14 \\
1.68\end{array}$ \\
\hline Total & 86.9 & 82.3 & 81.3 & 82.2 & 83.5 & 87.8 & 88.2 & 86.0 \\
\hline $\begin{array}{l}\mathrm{Si} \\
\mathrm{Ti} \\
\mathrm{Al} \\
\mathrm{Fe} \\
\mathrm{Mg} \\
\mathrm{Ca} \\
\mathrm{Ba} \\
\mathrm{Na} \\
\mathrm{K}\end{array}$ & $\begin{array}{c}29.2 \\
0.00 \\
6.99 \\
0.00 \\
0.88 \\
1.95 \\
0.00 \\
0.03 \\
0.42\end{array}$ & $\begin{array}{c}\text { Unit. } \\
28.7 \\
0.00 \\
7.42 \\
0.00 \\
1.06 \\
2.00 \\
0.00 \\
0.12 \\
0.57\end{array}$ & $\begin{array}{c}11 \text { Comp } \\
28.7 \\
0.00 \\
7.45 \\
0.00 \\
1.08 \\
2.00 \\
0.01 \\
0.13 \\
0.57\end{array}$ & $\begin{array}{c}\text { ition } \\
28.5 \\
0.00 \\
7.66 \\
0.00 \\
1.21 \\
2.03 \\
0.00 \\
0.11 \\
0.35\end{array}$ & $\begin{array}{c}\text { ed on } 7 \\
28.6 \\
0.00 \\
7.53 \\
0.00 \\
1.26 \\
1.99 \\
0.02 \\
0.09 \\
0.31\end{array}$ & $\begin{array}{c}(0) \\
29.4 \\
0.00 \\
6.81 \\
0.01 \\
0.64 \\
1.92 \\
0.09 \\
0.09 \\
0.71\end{array}$ & $\begin{array}{c}29.2 \\
0.00 \\
6.86 \\
0.11 \\
0.62 \\
1.90 \\
0.07 \\
0.21 \\
0.90\end{array}$ & $\begin{array}{c}29.1 \\
0.03 \\
6.97 \\
0.09 \\
0.64 \\
1.88 \\
0.04 \\
0.12 \\
0.95\end{array}$ \\
\hline \multicolumn{9}{|c|}{$3.85 \begin{array}{c}\mathrm{Si} /(\mathrm{Al}+\mathrm{Fe}) \\
3.72\end{array}$} \\
\hline & 1.15 & 1.09 & $\begin{array}{c}A 1+F e) \\
1.08\end{array}$ & $\begin{array}{r}\mathrm{Mg}+2 \mathrm{Ca} \\
1.11\end{array}$ & $\begin{array}{c}\mathrm{a}+\mathrm{Na}+\mathrm{K}) \\
1.09\end{array}$ & 1.12 & 1.11 & 1.14 \\
\hline $\begin{array}{l}\mathrm{K} \\
\mathrm{Na} \\
\mathrm{Ca}+\mathrm{Mg}\end{array}$ & $\begin{array}{r}12.8 \\
0.8 \\
86.4\end{array}$ & $\begin{array}{r}15.3 \\
3.1 \\
81.6\end{array}$ & $\begin{array}{c}1 \% \text { Exch } \\
15.0 \\
3.4 \\
81.6\end{array}$ & $\begin{array}{c}\text { geable } \\
9.4 \\
2.9 \\
87.6\end{array}$ & $\begin{array}{r}\text { tions } \\
8.6 \\
2.4 \\
89.0\end{array}$ & $\begin{array}{r}21.2 \\
2.8 \\
76.1\end{array}$ & $\begin{array}{r}24.8 \\
5.8 \\
69.4\end{array}$ & $\begin{array}{r}26.5 \\
3.4 \\
70.2\end{array}$ \\
\hline
\end{tabular}




\section{APPENDIX $F$ (cont)}

\section{Drill Hole USW G-2}

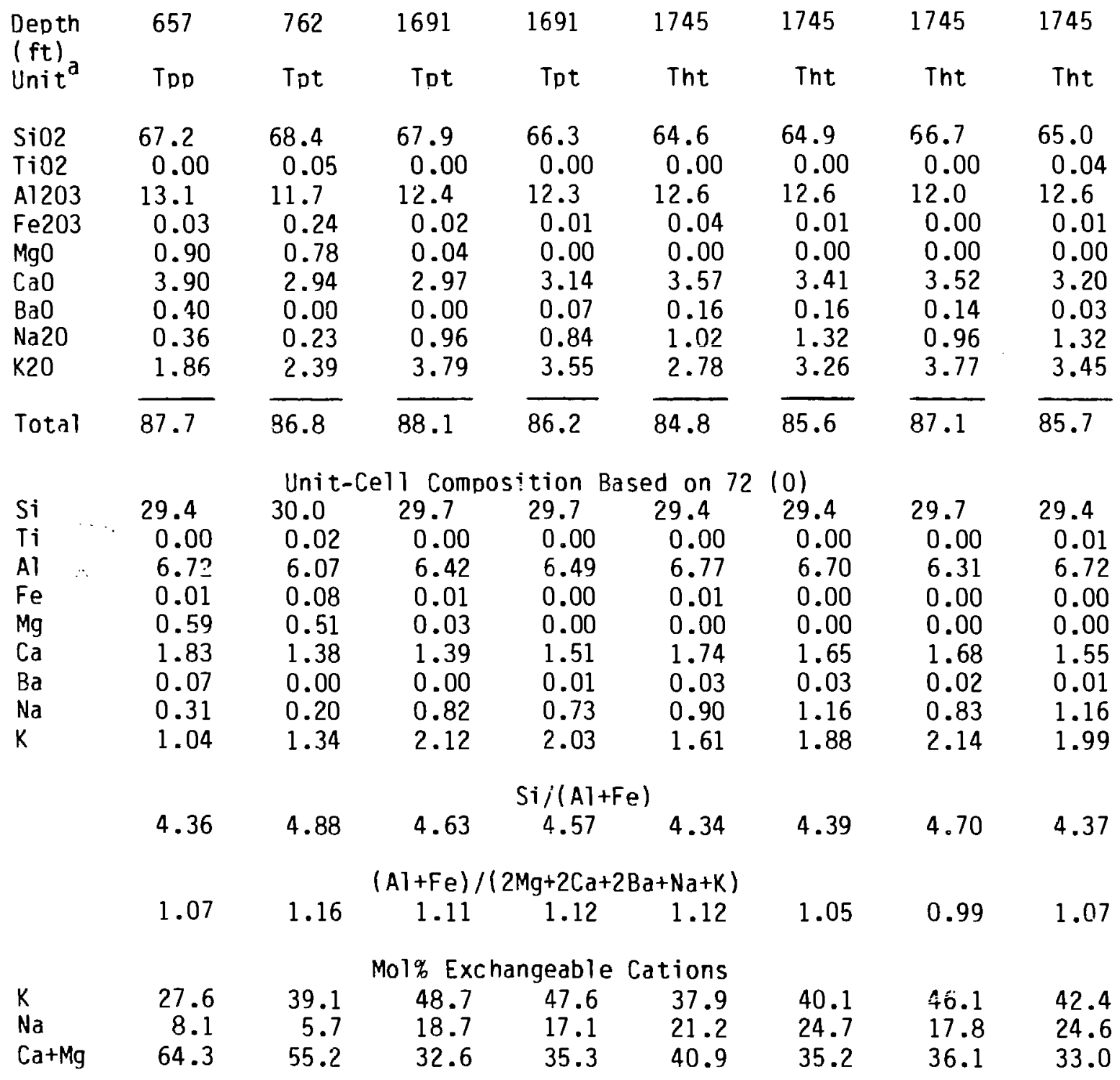




\section{APPENDIX $F$ (cont) \\ Drill Hole USW G-2}

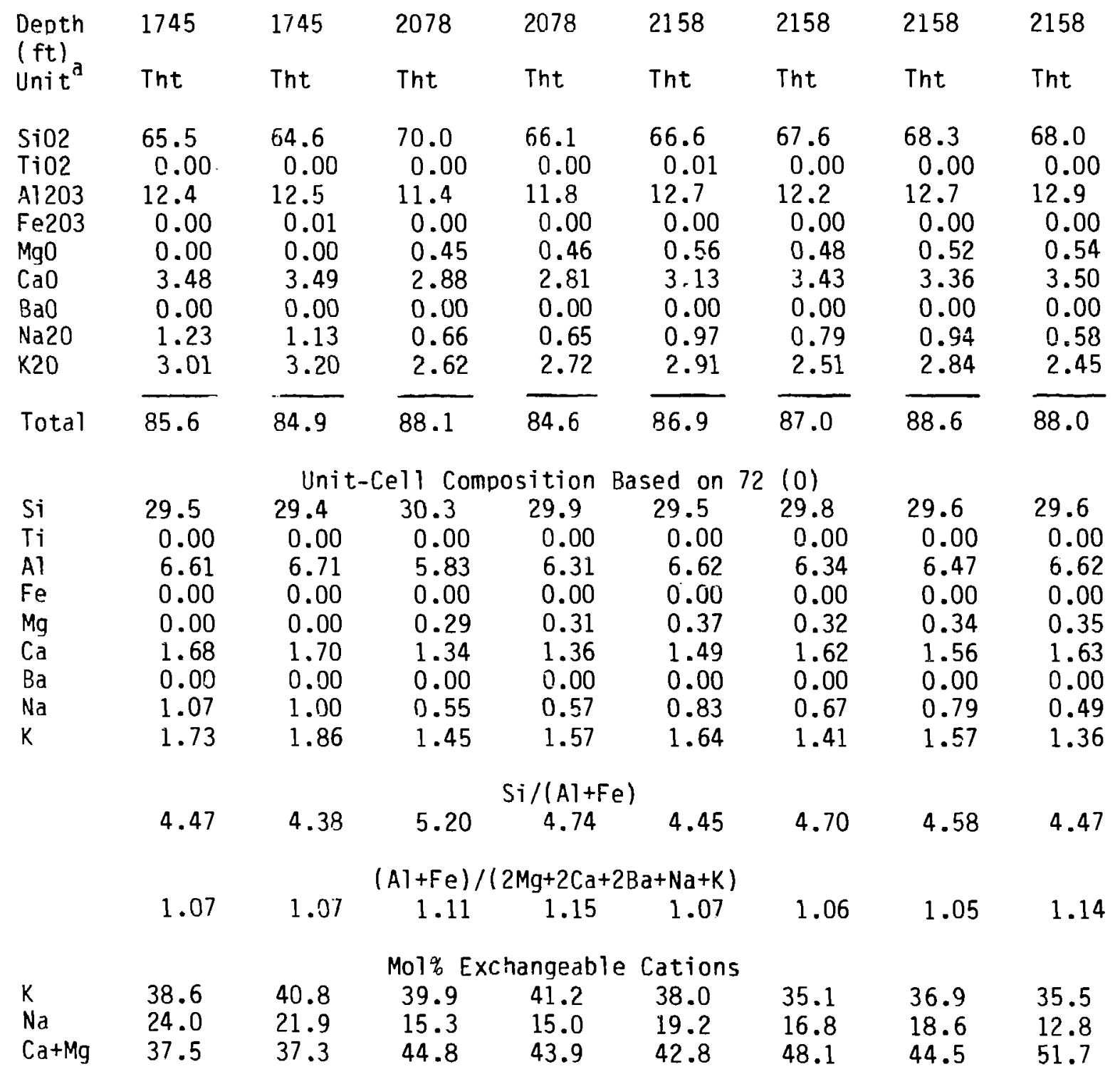




\section{APPENDIX F (cont) \\ Drill Hole USW G-2}

\begin{tabular}{|c|c|c|c|c|c|c|c|c|}
\hline $\begin{array}{l}\text { Depth } \\
\text { (ft) } \\
\text { Unit }\end{array}$ & 2158 & 2158 & 2325 & 2325 & 2430 & 2430 & 3067 & 3067 \\
\hline $\begin{array}{l}\mathrm{SiO} 2 \\
\mathrm{TiO} 02 \\
\mathrm{~A} 1203 \\
\mathrm{Fe} 203 \\
\mathrm{MgO} \\
\mathrm{CaO} \\
\mathrm{BaO} \\
\mathrm{Na} 20 \\
\mathrm{~K} 20\end{array}$ & $\begin{array}{c}68.6 \\
0.00 \\
11.4 \\
0.00 \\
0.41 \\
3.23 \\
0.00 \\
0.42 \\
2.23\end{array}$ & $\begin{array}{c}66.6 \\
0.00 \\
11.6 \\
0.00 \\
0.51 \\
3.23 \\
0.00 \\
0.77 \\
2.64\end{array}$ & $\begin{array}{c}69.4 \\
0.00 \\
12.0 \\
0.03 \\
0.30 \\
3.45 \\
0.00 \\
1.07 \\
2.36\end{array}$ & $\begin{array}{c}70.1 \\
0.00 \\
12.7 \\
0.03 \\
0.28 \\
3.53 \\
0.00 \\
0.95 \\
2.45\end{array}$ & $\begin{array}{c}70.2 \\
0.00 \\
11.6 \\
0.01 \\
0.36 \\
2.77 \\
0.15 \\
0.64 \\
2.81\end{array}$ & $\begin{array}{c}71.3 \\
0.00 \\
12.0 \\
0.00 \\
0.31 \\
3.08 \\
0.00 \\
0.72 \\
2.71\end{array}$ & $\begin{array}{c}74.1 \\
0.10 \\
11.4 \\
0.52 \\
0.00 \\
0.34 \\
0.11 \\
4.60 \\
3.04\end{array}$ & $\begin{array}{c}74.7 \\
0.10 \\
11.7 \\
0.73 \\
0.03 \\
0.32 \\
0.16 \\
4.66 \\
3.06\end{array}$ \\
\hline Total & 86.3 & 85.4 & 88.6 & 90.0 & 88.5 & 90.1 & 94.2 & 95.5 \\
\hline $\begin{array}{l}\mathrm{Si} \\
\mathrm{Ti} \\
\mathrm{Al} \\
\mathrm{Fe} \\
\mathrm{Mg} \\
\mathrm{Ca} \\
\mathrm{Ba} \\
\mathrm{Na} \\
\mathrm{K}\end{array}$ & $\begin{array}{l}30.2 \\
0.00 \\
5.94 \\
0.00 \\
0.27 \\
1.53 \\
0.00 \\
0.36 \\
1.25\end{array}$ & $\begin{array}{c}\text { Uni } \\
29.9 \\
0.00 \\
6.16 \\
0.00 \\
0.34 \\
1.55 \\
0.00 \\
0.67 \\
1.51\end{array}$ & $\begin{array}{c}\text { e11 Con } \\
30.0 \\
0.00 \\
6.11 \\
0.01 \\
0.19 \\
1.60 \\
0.00 \\
0.90 \\
1.30\end{array}$ & $\begin{array}{c}\text { sition } \\
29.8 \\
0.00 \\
6.35 \\
0.01 \\
0.18 \\
1.61 \\
0.00 \\
0.78 \\
1.33\end{array}$ & $\begin{array}{c}\text { sed on } \\
30.3 \\
0.00 \\
5.92 \\
0.00 \\
0.23 \\
1.28 \\
0.03 \\
0.54 \\
\vdots .55\end{array}$ & $\begin{array}{l}(0) \\
30.2 \\
0.00 \\
6.01 \\
0.00 \\
0.20 \\
1.40 \\
0.00 \\
0.59 \\
1.46\end{array}$ & $\begin{array}{l}30.3 \\
0.03 \\
5.50 \\
0.16 \\
0.00 \\
0.15 \\
0.02 \\
3.65 \\
1.59\end{array}$ & $\begin{array}{l}30.2 \\
0.03 \\
5.58 \\
0.22 \\
0.02 \\
0.14 \\
0.03 \\
3.66 \\
1.58\end{array}$ \\
\hline \multicolumn{9}{|c|}{$\mathrm{Si} /(\mathrm{Al}+\mathrm{Fe})$} \\
\hline \multicolumn{9}{|c|}{$(\mathrm{A}]+\mathrm{Fe}) /(2 \mathrm{Mg}+2 \mathrm{Ca}+2 \mathrm{Ba}+\mathrm{Na}+\mathrm{K})$} \\
\hline $\begin{array}{l}\mathrm{K} \\
\mathrm{Na} \\
\mathrm{Ca}+\mathrm{Mg}\end{array}$ & $\begin{array}{l}36.8 \\
10.5 \\
52.7\end{array}$ & $\begin{array}{l}37.1 \\
16.4 \\
46.5\end{array}$ & $\begin{array}{c}\text { Mo1\% E } \\
32.6 \\
22.5 \\
44.9\end{array}$ & $\begin{array}{c}\text { angeab } \\
34.1 \\
20.1 \\
45.8\end{array}$ & $\begin{array}{c}\text { Cations } \\
43.0 \\
14.9 \\
42.1\end{array}$ & $\begin{array}{l}40.1 \\
16.2 \\
43.7\end{array}$ & $\begin{array}{r}29.5 \\
67.8 \\
2.8\end{array}$ & $\begin{array}{r}29.3 \\
67.8 \\
2.9\end{array}$ \\
\hline
\end{tabular}




\section{APPENDIX $F$ (cont)}

Drill Hole USW G-2

\begin{tabular}{|c|c|c|c|c|c|c|c|c|}
\hline $\begin{array}{l}\text { Depth } \\
\text { (ft) } \\
\text { Unit }^{\mathrm{a}}\end{array}$ & 3067 & 3067 & 3067 & 3067 & 3192 & 3192 & 3192 & 3192 \\
\hline $\begin{array}{l}\mathrm{SiO} 2 \\
\mathrm{TiO} 2 \\
\mathrm{Al} 203 \\
\mathrm{Fe} 203 \\
\mathrm{MgO} \\
\mathrm{CaO} \\
\mathrm{BaO} \\
\mathrm{Na} 20 \\
\mathrm{~K} 20 \\
\text { Total }\end{array}$ & $\begin{array}{c}74.5 \\
0.07 \\
11.6 \\
0.80 \\
0.00 \\
0.29 \\
0.08 \\
4.48 \\
\frac{2.86}{94.7}\end{array}$ & $\begin{array}{c}73.4 \\
0.10 \\
11.6 \\
0.18 \\
0.00 \\
0.29 \\
0.11 \\
4.74 \\
3.17 \\
93.6\end{array}$ & $\begin{array}{c}74.3 \\
0.09 \\
11.7 \\
0.71 \\
0.00 \\
0.34 \\
0.18 \\
4.77 \\
\frac{2.87}{95.0}\end{array}$ & $\begin{array}{c}69.7 \\
0.00 \\
10.5 \\
0.00 \\
0.00 \\
2.70 \\
0.18 \\
2.48 \\
\frac{0.51}{86.1}\end{array}$ & $\begin{array}{c}63.2 \\
0.00 \\
12.2 \\
0.05 \\
0.00 \\
2.06 \\
0.00 \\
3.20 \\
1.85 \\
82.6\end{array}$ & $\begin{array}{c}64.2 \\
0.03 \\
12.5 \\
0.18 \\
0.02 \\
2.25 \\
0.00 \\
3.26 \\
\frac{2.15}{84.6}\end{array}$ & $\begin{array}{c}63.7 \\
0.03 \\
12.2 \\
0.11 \\
0.00 \\
2.05 \\
0.00 \\
2.51 \\
\frac{1.61}{83.3}\end{array}$ & $\begin{array}{c}65.0 \\
0.00 \\
12.5 \\
0.11 \\
0.02 \\
1.91 \\
0.00 \\
3.61 \\
\frac{1.77}{84.9}\end{array}$ \\
\hline $\begin{array}{l}\mathrm{Si} \\
\mathrm{Ti} \\
\mathrm{Al} \\
\mathrm{Fe} \\
\mathrm{Mg} \\
\mathrm{Ca} \\
\mathrm{Ba} \\
\mathrm{Na} \\
\mathrm{K}\end{array}$ & $\begin{array}{l}30.3 \\
0.02 \\
5.56 \\
0.24 \\
0.00 \\
0.13 \\
0.01 \\
3.53 \\
1.48\end{array}$ & $\begin{array}{c}\text { Un } \\
30.3 \\
0.03 \\
5.64 \\
0.06 \\
0.00 \\
0.13 \\
0.02 \\
3.79 \\
1.67\end{array}$ & $\begin{array}{c}\text { Cel1 C } \\
30.2 \\
0.03 \\
5.61 \\
0.22 \\
0.00 \\
0.15 \\
0.03 \\
3.76 \\
1.49\end{array}$ & $\begin{array}{c}\text { osition } \\
30.7 \\
0.00 \\
5.45 \\
0.00 \\
0.00 \\
1.27 \\
0.03 \\
2.12 \\
0.29\end{array}$ & $\begin{array}{c}\text { 3ased on } \\
29.4 \\
0.00 \\
6.70 \\
0.02 \\
0.00 \\
1.03 \\
0.00 \\
2.89 \\
1.10\end{array}$ & $\begin{array}{c}72(0) \\
29.3 \\
0.01 \\
6.73 \\
0.06 \\
0.01 \\
1.10 \\
0 .(00 \\
2.89 \\
1.25\end{array}$ & $\begin{array}{l}29.4 \\
0.01 \\
6.64 \\
0.04 \\
0.00 \\
1.01 \\
0.00 \\
3.23 \\
0.95\end{array}$ & $\begin{array}{l}29.4 \\
0.00 \\
6.68 \\
0.04 \\
0.01 \\
0.93 \\
0.00 \\
3.17 \\
1.02\end{array}$ \\
\hline \multicolumn{9}{|c|}{$\mathrm{Si} /(\mathrm{Al}+\mathrm{Fe})$} \\
\hline & 1.10 & 0.99 & $\begin{array}{r}(A)+F e \\
1.04\end{array}$ & $\begin{array}{c}2 \mathrm{Mg}+2 \mathrm{Ca} \\
\quad 1.09\end{array}$ & $\begin{aligned} 2 E & \approx+N a+K \\
& 1.11\end{aligned}$ & 1.07 & 1.08 & 1.11 \\
\hline $\begin{array}{l}\mathrm{K} \\
\mathrm{Na} \\
\mathrm{Ca}+\mathrm{Mg}\end{array}$ & $\begin{array}{r}28.9 \\
68.7 \\
2.5\end{array}$ & $\begin{array}{r}29.9 \\
67.8 \\
2.3\end{array}$ & $\begin{array}{r}\text { Mol\% } \\
27.6 \\
69.7 \\
2.7\end{array}$ & $\begin{array}{c}\text { hangeab } \\
7.8 \\
57.6 \\
34.6\end{array}$ & $\begin{array}{l}\text { Catio } \\
21.9 \\
57.6 \\
20.5\end{array}$ & $\begin{array}{l}23.8 \\
54.9 \\
21.2\end{array}$ & $\begin{array}{l}18.3 \\
62.2 \\
19.5\end{array}$ & $\begin{array}{l}19.9 \\
61.8 \\
18.3\end{array}$ \\
\hline
\end{tabular}


Drill Hole USW G-2

\begin{tabular}{|c|c|c|c|c|c|c|}
\hline \multirow{2}{*}{$\begin{array}{l}\text { Depth } \\
\text { (ft) } \\
\text { Unit }\end{array}$} & 3192 & 3192 & 3192 & 3192 & 3250 & 3250 \\
\hline & $T C D$ & TCD & TCD & TCD & TCD & $T C D$ \\
\hline $\begin{array}{l}\mathrm{SiO} 02 \\
\mathrm{Ti} 02 \\
\mathrm{~A} 1203 \\
\mathrm{Fe} 203 \\
\mathrm{MgO} \\
\mathrm{Ca} 0 \\
\mathrm{BaO} \\
\mathrm{Na} 20 \\
\mathrm{~K} 20\end{array}$ & $\begin{array}{c}63.0 \\
0.05 \\
12.8 \\
0.54 \\
0.05 \\
2.16 \\
0.17 \\
3.33 \\
1.73\end{array}$ & $\begin{array}{c}66.0 \\
0.04 \\
12.6 \\
0.17 \\
0.00 \\
2.09 \\
0.00 \\
3.26 \\
2.00\end{array}$ & $\begin{array}{c}66.2 \\
0.00 \\
12.8 \\
0.17 \\
0.05 \\
2.43 \\
0.05 \\
3.08 \\
1.99\end{array}$ & $\begin{array}{c}65.4 \\
0.00 \\
12.2 \\
0.29 \\
0.00 \\
1.72 \\
0.00 \\
3.32 \\
2.32\end{array}$ & $\begin{array}{c}66.9 \\
0.00 \\
11.6 \\
0.08 \\
0.00 \\
2.33 \\
0.02 \\
3.73 \\
0.04\end{array}$ & $\begin{array}{c}68.2 \\
0.00 \\
12.0 \\
0.13 \\
0.02 \\
2.37 \\
0.04 \\
4.20 \\
0.13\end{array}$ \\
\hline Total & 83.8 & 86.2 & 86.8 & 85.3 & 84.7 & 87.1 \\
\hline $\begin{array}{l}\mathrm{Si} \\
\mathrm{Ti} \\
\mathrm{Al} \\
\mathrm{Fe} \\
\mathrm{Mg} \\
\mathrm{Ca} \\
\mathrm{Ba} \\
\mathrm{Na} \\
\mathrm{Ka}\end{array}$ & $\begin{array}{c}\text { Un } \\
29.1 \\
0.02 \\
6.96 \\
0.19 \\
0.03 \\
1.07 \\
0.03 \\
2.98 \\
1.02\end{array}$ & $\begin{array}{c}\text { Cell C } \\
29.5 \\
0.01 \\
6.63 \\
0.06 \\
0.00 \\
1.00 \\
0.00 \\
2.82 \\
1.14\end{array}$ & $\begin{array}{c}\text { osition } \\
29.4 \\
0.00 \\
6.70 \\
0.06 \\
0.03 \\
1.16 \\
0.01 \\
2.65 \\
1.13\end{array}$ & $\begin{array}{c}\text { ased on } \\
29.6 \\
0.00 \\
6.50 \\
0.10 \\
0.00 \\
0.83 \\
0.00 \\
2.91 \\
1.34\end{array}$ & $\begin{array}{c}70) \\
30.0 \\
0.00 \\
6.13 \\
0.03 \\
0.00 \\
1.12 \\
0.00 \\
3.24 \\
0.02\end{array}$ & $\begin{array}{c}29.8 \\
0.00 \\
6.19 \\
0.04 \\
0.01 \\
1.11 \\
0.01 \\
3.56 \\
0.07\end{array}$ \\
\hline \multicolumn{6}{|c|}{$\mathrm{Si} /(\mathrm{Al}+\mathrm{Fe})$} & 4.79 \\
\hline & 1.14 & $\begin{array}{r}1 \mathrm{~A} \\
1.12\end{array}$ & $\begin{array}{c}\text { e) } /(2 \mathrm{Mg} \\
1.09\end{array}$ & $\begin{array}{r}2 \mathrm{Ca}+2 \mathrm{Ba}+ \\
1.12\end{array}$ & $\begin{array}{l}a+k) \\
1.12\end{array}$ & 1.06 \\
\hline $\begin{array}{l}\mathrm{K} \\
\mathrm{Na} \\
\mathrm{Ca}+\mathrm{Mg}\end{array}$ & $\begin{array}{l}20.0 \\
58.4 \\
21.6\end{array}$ & $\begin{array}{l}\text { Mol } \\
23.0 \\
56.9 \\
20.2\end{array}$ & $\begin{array}{l}\text { xchange } \\
22.7 \\
53.4 \\
23.9\end{array}$ & $\begin{array}{c}\text { ble Cati } \\
26.3 \\
57.3 \\
16.4\end{array}$ & ns $\begin{array}{r}0.5 \\
74.0 \\
25.5\end{array}$ & $\begin{array}{r}1.5 \\
74.9 \\
23.6\end{array}$ \\
\hline
\end{tabular}




\section{APPENDIX $F$ (cont) \\ Drill Hole USW G-3}

\begin{tabular}{|c|c|c|c|c|c|c|c|c|}
\hline $\begin{array}{l}\text { Depth } \\
\text { (ft) } \\
\text { Unit }^{a}\end{array}$ & 1195 & 1195 & 1195 & 1195 & 1195 & 1195 & 1874 & 1874 \\
\hline $\begin{array}{l}\mathrm{SiO} 2 \\
\mathrm{TiO} 02 \\
\mathrm{~A} 1203 \\
\mathrm{Fe} 203 \\
\mathrm{MgO} \\
\mathrm{CaO} \\
\mathrm{BaO} \\
\mathrm{Na} 20 \\
\mathrm{~K} 20\end{array}$ & $\begin{array}{c}64.7 \\
0.03 \\
13.2 \\
0.30 \\
0.91 \\
4.28 \\
0.04 \\
0.70 \\
0.36\end{array}$ & $\begin{array}{c}67.0 \\
0.04 \\
12.3 \\
0.25 \\
0.72 \\
4.00 \\
0.13 \\
0.77 \\
0.78\end{array}$ & $\begin{array}{c}62.6 \\
0.02 \\
12.4 \\
0.00 \\
0.85 \\
4.03 \\
0.00 \\
0.54 \\
0.48\end{array}$ & $\begin{array}{c}64.9 \\
0.00 \\
12.1 \\
0.00 \\
0.79 \\
3.96 \\
0.01 \\
0.59 \\
0.64\end{array}$ & $\begin{array}{c}63.1 \\
0.05 \\
11.8 \\
0.83 \\
0.93 \\
3.85 \\
0.19 \\
0.48 \\
0.94\end{array}$ & $\begin{array}{c}70.0 \\
0.00 \\
12.4 \\
0.00 \\
0.56 \\
3.94 \\
0.00 \\
0.70 \\
1.19\end{array}$ & $\begin{array}{c}69.5 \\
0.00 \\
12.1 \\
0.04 \\
0.00 \\
1.62 \\
0.00 \\
1.46 \\
4.91\end{array}$ & $\begin{array}{c}66.5 \\
0.00 \\
11.2 \\
0.02 \\
0.00 \\
1.69 \\
0.00 \\
1.76 \\
3.95\end{array}$ \\
\hline Total & 84.5 & 86.0 & 80.9 & 82.9 & 32.2 & 88.8 & 89.7 & 85.1 \\
\hline $\begin{array}{l}\mathrm{Si} \\
\mathrm{Ti} \\
\mathrm{Al} \\
\mathrm{Fe} \\
\mathrm{Mg} \\
\mathrm{Ca} \\
\mathrm{Ba} \\
\mathrm{Na} \\
\mathrm{K}\end{array}$ & $\begin{array}{l}29.1 \\
0.01 \\
6.99 \\
0.10 \\
0.61 \\
2.06 \\
0.01 \\
0.61 \\
0.21\end{array}$ & $\begin{array}{c}\text { Un } \\
29.6 \\
0.01 \\
6.44 \\
0.08 \\
0.47 \\
1.90 \\
0.02 \\
0.66 \\
0.44\end{array}$ & $\begin{array}{c}\text { Cel1 C C } \\
29.4 \\
0.01 \\
6.84 \\
0.00 \\
0.59 \\
2.03 \\
0.00 \\
0.49 \\
0.29\end{array}$ & $\begin{array}{c}\text { sition } \\
29.7 \\
0.00 \\
6.51 \\
0.00 \\
0.54 \\
1.94 \\
0.00 \\
0.52 \\
0.37\end{array}$ & $\begin{array}{c}\text { Based on } \\
29.4 \\
0.02 \\
6.46 \\
0.29 \\
0.64 \\
1.92 \\
0.03 \\
0.43 \\
0.56\end{array}$ & $\begin{array}{c}72(0) \\
29.9 \\
0.00 \\
6.24 \\
0.00 \\
0.36 \\
1.80 \\
0.00 \\
0.58 \\
0.65\end{array}$ & $\begin{array}{c}30.0 \\
0.00 \\
6.18 \\
0.01 \\
0.00 \\
0.75 \\
0.00 \\
1.22 \\
2.70\end{array}$ & $\begin{array}{c}30.1 \\
0.00 \\
5.99 \\
0.01 \\
0.00 \\
0.82 \\
0.00 \\
1.55 \\
2.28\end{array}$ \\
\hline \multicolumn{9}{|c|}{$\mathrm{Si} /(\mathrm{Al}+\mathrm{Fe})$} \\
\hline & 1.15 & 1.11 & $\begin{array}{r}(\mathrm{A})+\mathrm{Fe} \\
1.14\end{array}$ & $\begin{array}{c}2 M g+2 C \\
1.11\end{array}$ & $\begin{array}{c}2 \mathrm{Ba}+\mathrm{Na}+\mathrm{K} \\
1.09\end{array}$ & 1.12 & 1.14 & 1.09 \\
\hline $\begin{array}{l}\mathrm{K} \\
\mathrm{Na} \\
\mathrm{Ca}+\mathrm{Mg}\end{array}$ & $\begin{array}{r}5.9 \\
17.5 \\
76.6\end{array}$ & $\begin{array}{l}12.7 \\
19.0 \\
68.3\end{array}$ & $\begin{array}{c}\text { Mol\% E } \\
8.5 \\
14.5 \\
77.1\end{array}$ & $\begin{array}{l}\text { angeab } \\
11.1 \\
15.5 \\
73.4\end{array}$ & $\begin{array}{c}\text { Cations } \\
15.7 \\
12.2 \\
72.1\end{array}$ & $\begin{array}{l}19.1 \\
17.1 \\
63.7\end{array}$ & $\begin{array}{l}57.8 \\
26.1 \\
16.0\end{array}$ & $\begin{array}{l}49.1 \\
33.3 \\
17.6\end{array}$ \\
\hline
\end{tabular}


APPENDIX $F$ (cont)

Drill Hole USH G-3

\begin{tabular}{|c|c|c|c|c|c|c|c|c|}
\hline $\begin{array}{l}\text { Dinth } \\
\text { it } \\
\text { Unic }\end{array}$ & 1874 & 1874 & 1874 & 1874 & 1874 & 1874 & 1874 & 1986 \\
\hline $\begin{array}{l}\mathrm{SiO} 2 \\
\mathrm{TiO} 2 \\
\mathrm{Al} 203 \\
\mathrm{Fe} 203 \\
\mathrm{MgO} \\
\mathrm{CaO} \\
\mathrm{BaO} \\
\mathrm{Na} 20 \\
\mathrm{~K} 20\end{array}$ & $\begin{array}{c}67.7 \\
0.00 \\
12.0 \\
0.05 \\
0.00 \\
1.65 \\
0.00 \\
1.58 \\
4.61\end{array}$ & $\begin{array}{c}67.3 \\
0.01 \\
12.0 \\
0.05 \\
0.00 \\
1.57 \\
0.00 \\
1.84 \\
5.04\end{array}$ & $\begin{array}{c}67.6 \\
0.04 \\
12.0 \\
0.09 \\
0.00 \\
1.84 \\
0.00 \\
1.64 \\
5.44\end{array}$ & $\begin{array}{c}65.9 \\
0.00 \\
11.5 \\
0.50 \\
0.00 \\
1.59 \\
0.00 \\
1.53 \\
4.61\end{array}$ & $\begin{array}{c}68.2 \\
0.00 \\
11.9 \\
0.00 \\
0.00 \\
1.66 \\
0.00 \\
1.68 \\
4.30\end{array}$ & $\begin{array}{c}68.2 \\
0.00 \\
11.9 \\
0.00 \\
0.00 \\
1.95 \\
0.00 \\
1.69 \\
5.20\end{array}$ & $\begin{array}{c}69.0 \\
0.03 \\
11.8 \\
0.03 \\
0.00 \\
1.59 \\
0.00 \\
1.52 \\
5.05\end{array}$ & $\begin{array}{c}64.2 \\
0.00 \\
11.4 \\
0.00 \\
0.00 \\
0.59 \\
0.00 \\
1.65 \\
6.51\end{array}$ \\
\hline Total & 87.6 & 87.8 & 88.6 & 85.2 & 87.8 & 88.9 & 89.0 & 84.4 \\
\hline $\begin{array}{l}\mathrm{Si} \\
\mathrm{Ti} \\
\mathrm{Al} \\
\mathrm{Fe} \\
\mathrm{Mg} \\
\mathrm{Ca} \\
\mathrm{Ba} \\
\mathrm{Na} \\
\mathrm{K}\end{array}$ & $\begin{array}{l}29.9 \\
0.00 \\
6.25 \\
0.02 \\
0.00 \\
0.78 \\
0.00 \\
1.35 \\
2.60\end{array}$ & $\begin{array}{l}\text { Un } \\
29.8 \\
0.00 \\
6.26 \\
0.02 \\
0.00 \\
0.75 \\
0.00 \\
1.58 \\
2.85\end{array}$ & $\begin{array}{c}\text { Cel1 Cc } \\
29.8 \\
0.01 \\
6.22 \\
0.03 \\
0.00 \\
0.87 \\
0.00 \\
1.40 \\
3.06\end{array}$ & $\begin{array}{c}\text { osition } \\
30.0 \\
0.00 \\
6.18 \\
0.00 \\
0.00 \\
0.77 \\
0.00 \\
1.35 \\
2.67\end{array}$ & $\begin{array}{c}\text { Based on } \\
30.0 \\
0.00 \\
6.19 \\
0.00 \\
0.00 \\
0.78 \\
0.00 \\
1.43 \\
2.41\end{array}$ & $\begin{array}{c}72(0) \\
29.9 \\
0.00 \\
6.14 \\
0.00 \\
0.00 \\
0.91 \\
0.00 \\
1.43 \\
2.90\end{array}$ & $\begin{array}{l}30.0 \\
0.01 \\
6.07 \\
0.01 \\
0.00 \\
0.74 \\
0.00 \\
1.28 \\
2.81\end{array}$ & $\begin{array}{l}29.8 \\
0.00 \\
6.24 \\
0.00 \\
0.00 \\
0.29 \\
0.00 \\
1.49 \\
3.86\end{array}$ \\
\hline \multicolumn{9}{|c|}{$\mathrm{Si} /(\mathrm{Al}+\mathrm{Fe})$} \\
\hline$(\mathrm{A}]+\mathrm{Fe}) /(2 \mathrm{Mg}+2 \mathrm{C} a+2 \mathrm{Ba}+\mathrm{Na}+\mathrm{K})$ & 1.14 & 1.06 & $\begin{array}{r}(A]+F e) \\
1.01\end{array}$ & $\begin{array}{c}\mathrm{Mg}+2 \mathrm{Ca}+ \\
1.11\end{array}$ & $\begin{array}{c}\mathrm{Ba}+\mathrm{Na}+\mathrm{K}) \\
1.15\end{array}$ & 1.00 & 1.09 & 1.05 \\
\hline $\begin{array}{l}\mathrm{K} \\
\mathrm{Na} \\
\mathrm{Ca}+\mathrm{Mg}\end{array}$ & $\begin{array}{l}54.9 \\
28.6 \\
16.5\end{array}$ & $\begin{array}{l}55.1 \\
30.5 \\
14.4\end{array}$ & $\begin{array}{c}\text { Mol\% E } \\
57.4 \\
26.3 \\
16.3\end{array}$ & $\begin{array}{c}\text { langeab } \\
55.7 \\
28.1 \\
16.1\end{array}$ & $\begin{array}{c}\text { Cations } \\
52.1 \\
31.0 \\
16.9\end{array}$ & $\begin{array}{l}55.3 \\
27.3 \\
17.4\end{array}$ & $\begin{array}{l}58.1 \\
26.6 \\
15.4\end{array}$ & $\begin{array}{r}68.4 \\
26.4 \\
5.2\end{array}$ \\
\hline
\end{tabular}


Orill Hole USW G-3

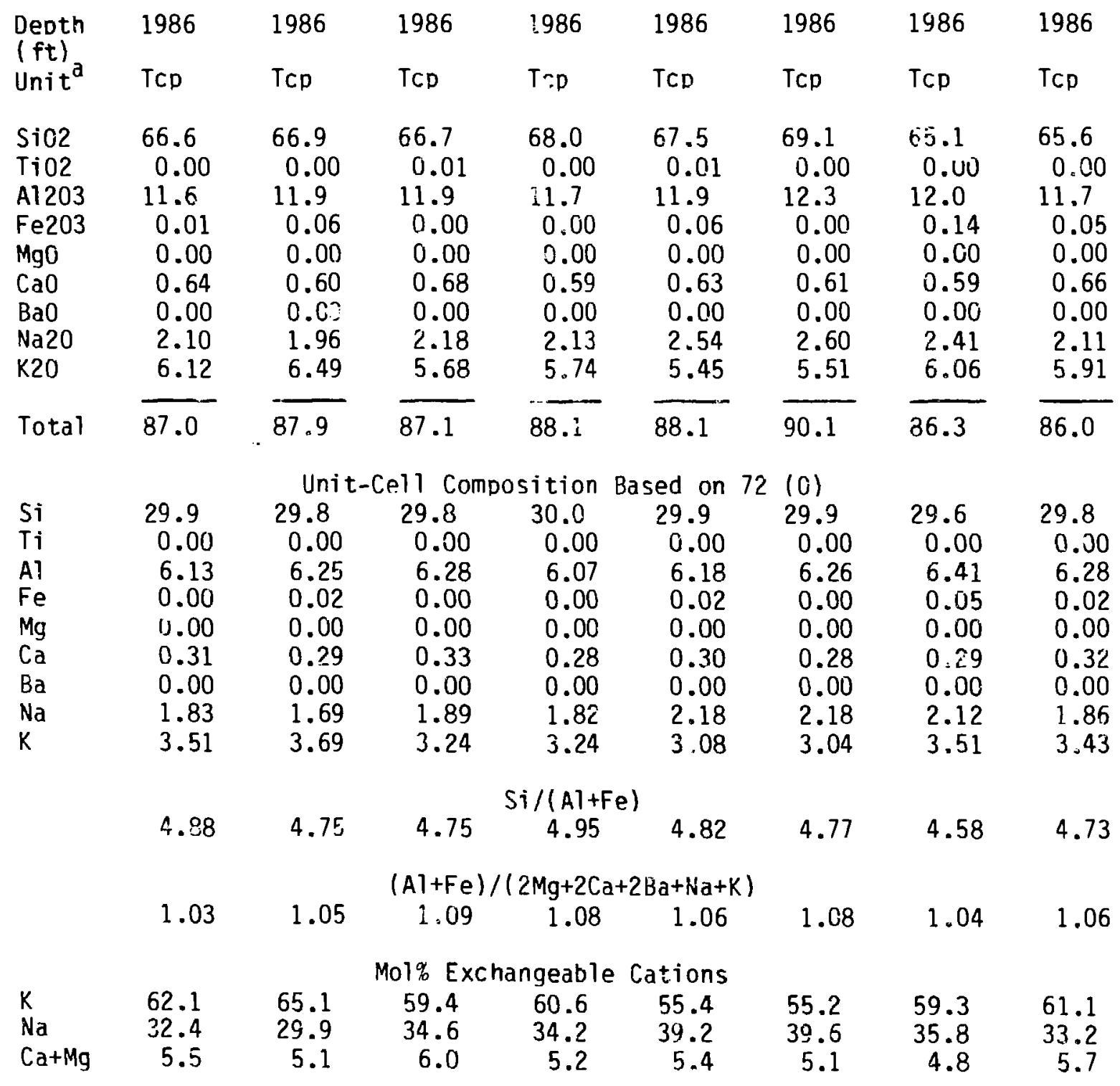




\section{APPENDIX $F$ (cont)}

Drill Hole USW G-3

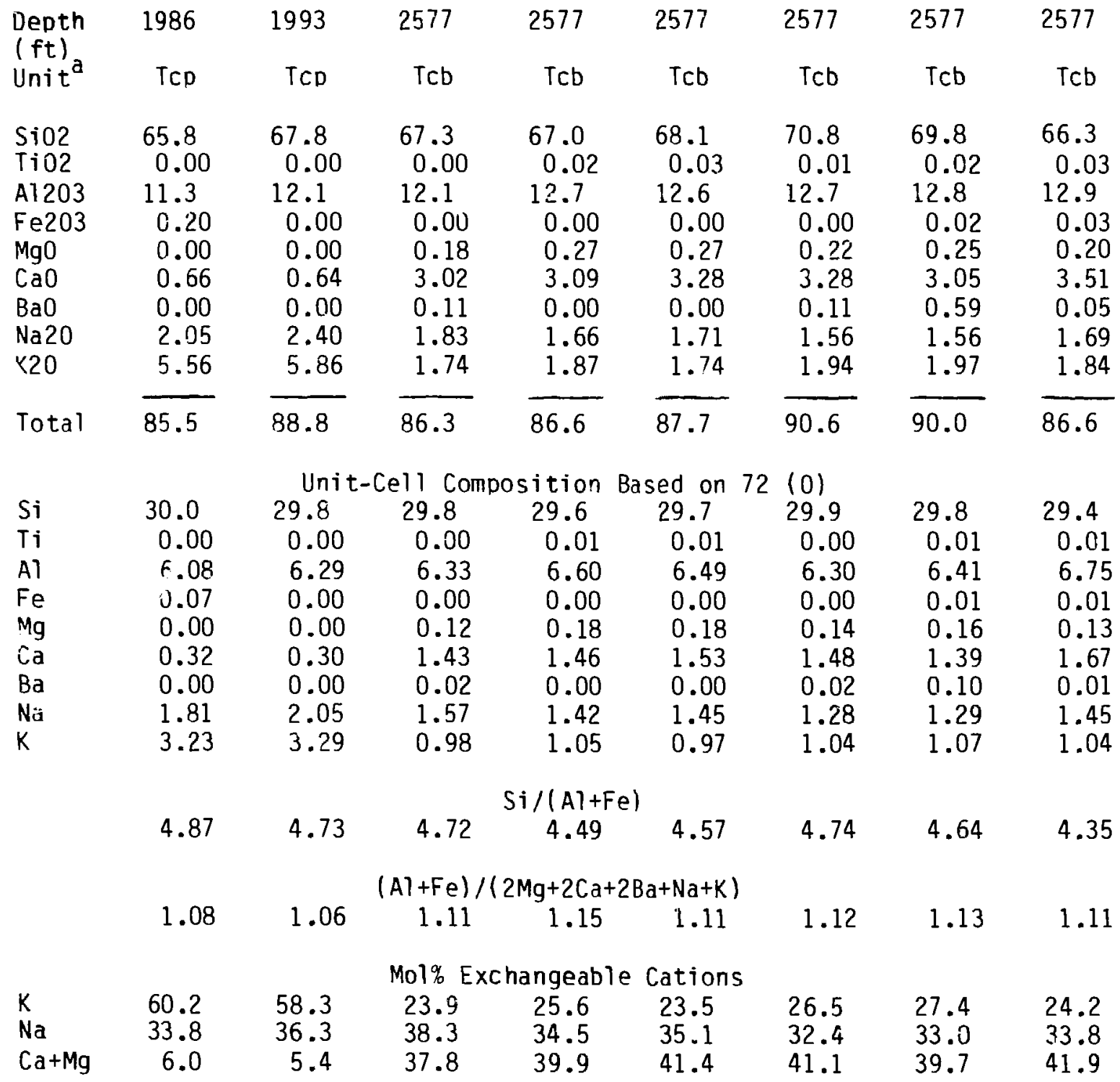




\section{APPENDIX $F$ (cont)}

Drill Hole USW G-3

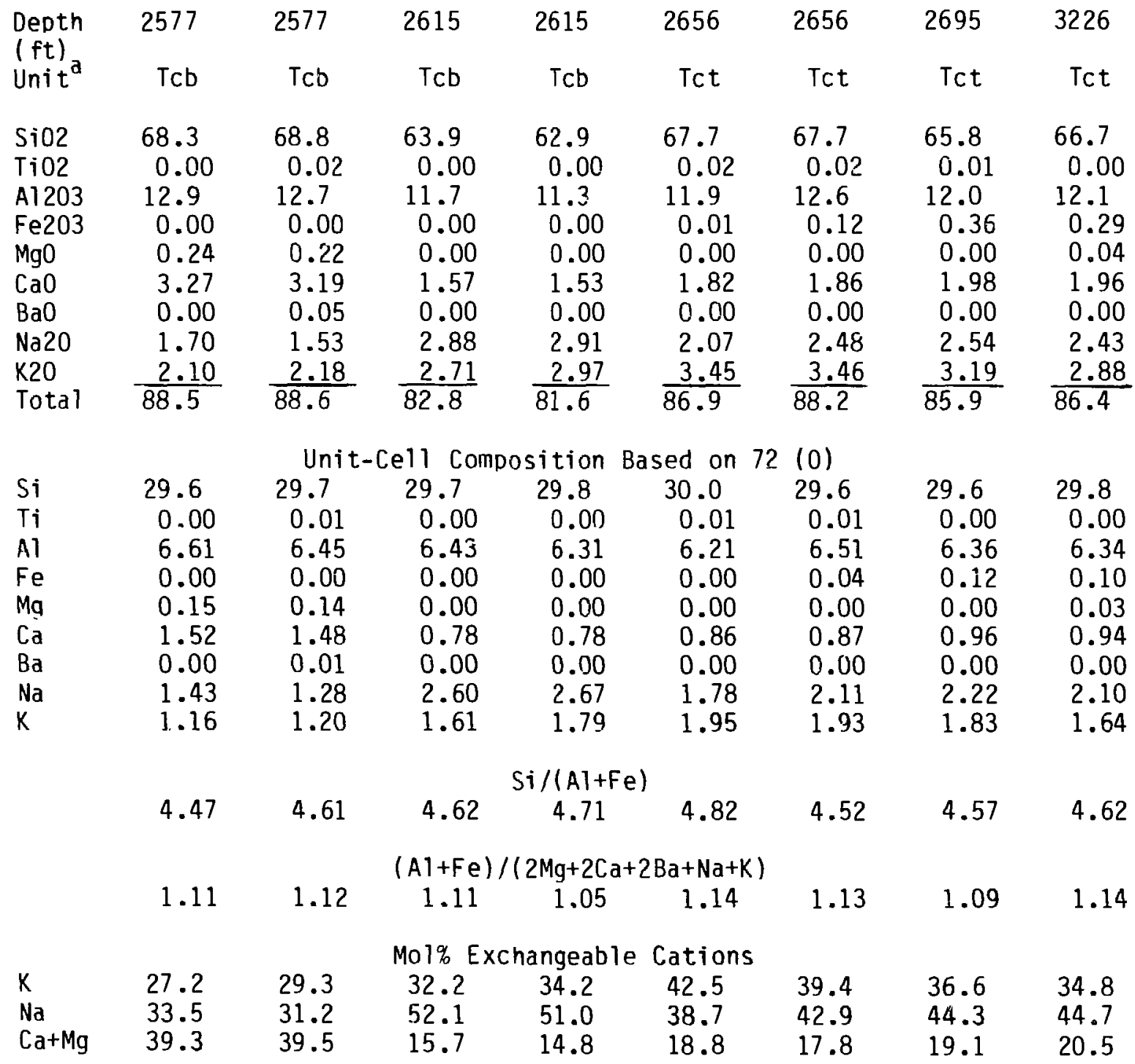




\section{APPENDIX $F$ (cont)}

Drill Hole USW G-3

\begin{tabular}{|c|c|c|c|c|c|c|c|c|}
\hline $\begin{array}{l}\text { Depth } \\
\text { (ft) } \\
\text { Unit }\end{array}$ & 3226 & 3475 & 3589 & 3589 & 3589 & $\begin{array}{r}3672 \\
\text { Tct }\end{array}$ & 3672 & 3672 \\
\hline $\begin{array}{l}\mathrm{Si02} \\
\mathrm{TiO} 2 \\
\mathrm{Al} 203 \\
\mathrm{Fe} 203 \\
\mathrm{MgO} \\
\mathrm{CaO} \\
\mathrm{BaO} \\
\mathrm{Na} 20 \\
\mathrm{~K} 20\end{array}$ & $\begin{array}{c}65.8 \\
0.00 \\
12.1 \\
0.68 \\
0.03 \\
1.91 \\
0.00 \\
2.78 \\
2.93\end{array}$ & $\begin{array}{c}66.9 \\
0.07 \\
12.3 \\
0.07 \\
0.30 \\
1.19 \\
0.07 \\
2.89 \\
3.57\end{array}$ & $\begin{array}{c}65.1 \\
0.00 \\
11.4 \\
0.51 \\
0.12 \\
0.79 \\
0.19 \\
2.23 \\
5.55\end{array}$ & $\begin{array}{c}67.0 \\
0.00 \\
11.4 \\
0.26 \\
0.00 \\
0.88 \\
0.13 \\
2.20 \\
4.47\end{array}$ & $\begin{array}{c}64.7 \\
0.00 \\
12.2 \\
0.00 \\
0.00 \\
1.19 \\
0.10 \\
2.42 \\
4.38\end{array}$ & $\begin{array}{c}64.8 \\
0.00 \\
12.3 \\
0.00 \\
0.00 \\
1.12 \\
0.13 \\
2.80 \\
3.85\end{array}$ & $\begin{array}{c}64.4 \\
0.00 \\
11.8 \\
0.22 \\
0.04 \\
1.07 \\
0.00 \\
2.98 \\
3.93\end{array}$ & $\begin{array}{c}64.7 \\
0.00 \\
12.7 \\
0.00 \\
0.02 \\
1.09 \\
0.32 \\
2.81 \\
4.46\end{array}$ \\
\hline Total & 86.3 & 87.3 & 85.9 & 86.3 & 85.0 & 84.9 & 84.5 & 86.1 \\
\hline \multicolumn{9}{|c|}{ 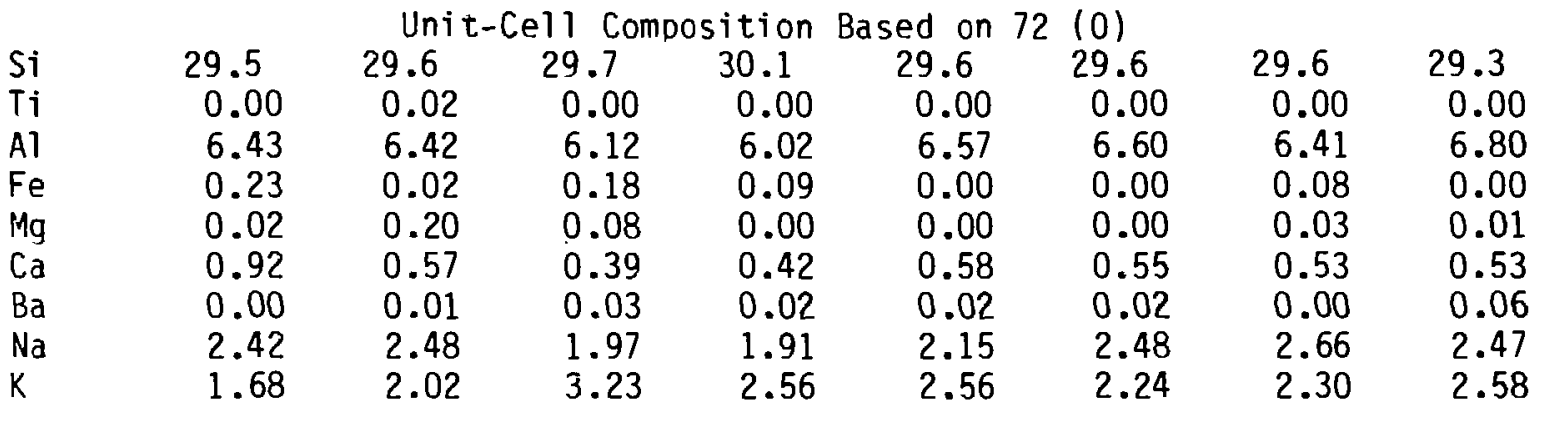 } \\
\hline & 4.44 & 4.60 & 4.72 & $\begin{array}{c}\mathrm{Si} /(\mathrm{Al}+\mathrm{F} \\
4.93\end{array}$ & 4.50 & 4.49 & 4.56 & 4.32 \\
\hline & 1.11 & 1.06 & $\begin{array}{c}(A]+F e) \\
1.01\end{array}$ & $\begin{array}{c}2 \mathrm{Mg}+2 \mathrm{Ca} \\
1.14\end{array}$ & $\begin{array}{c}-2 \mathrm{Ba}+\mathrm{Na}+\mathrm{K}) \\
1.11\end{array}$ & 1.12 & 1.07 & 1.09 \\
\hline $\begin{array}{l}\mathrm{K} \\
\mathrm{Na} \\
\mathrm{Ca}+\mathrm{Mg}\end{array}$ & $\begin{array}{l}33.3 \\
48.0 \\
18.6\end{array}$ & $\begin{array}{l}38.3 \\
47.2 \\
14.5\end{array}$ & $\begin{array}{c}\text { Mol\% Exc } \\
57.0 \\
34.8 \\
8.2\end{array}$ & $\begin{array}{c}\text { angeable } \\
52.3 \\
39.1 \\
8.6\end{array}$ & $\begin{array}{c}\text { Cations } \\
48.4 \\
40.6 \\
11.0\end{array}$ & $\begin{array}{l}42.6 \\
47.0 \\
10.4\end{array}$ & $\begin{array}{l}41.8 \\
48.2 \\
10.1\end{array}$ & $\begin{array}{r}46.1 \\
44.2 \\
9.7\end{array}$ \\
\hline
\end{tabular}


APPENDIX F (cont)

Dri11 Hole USW G-3

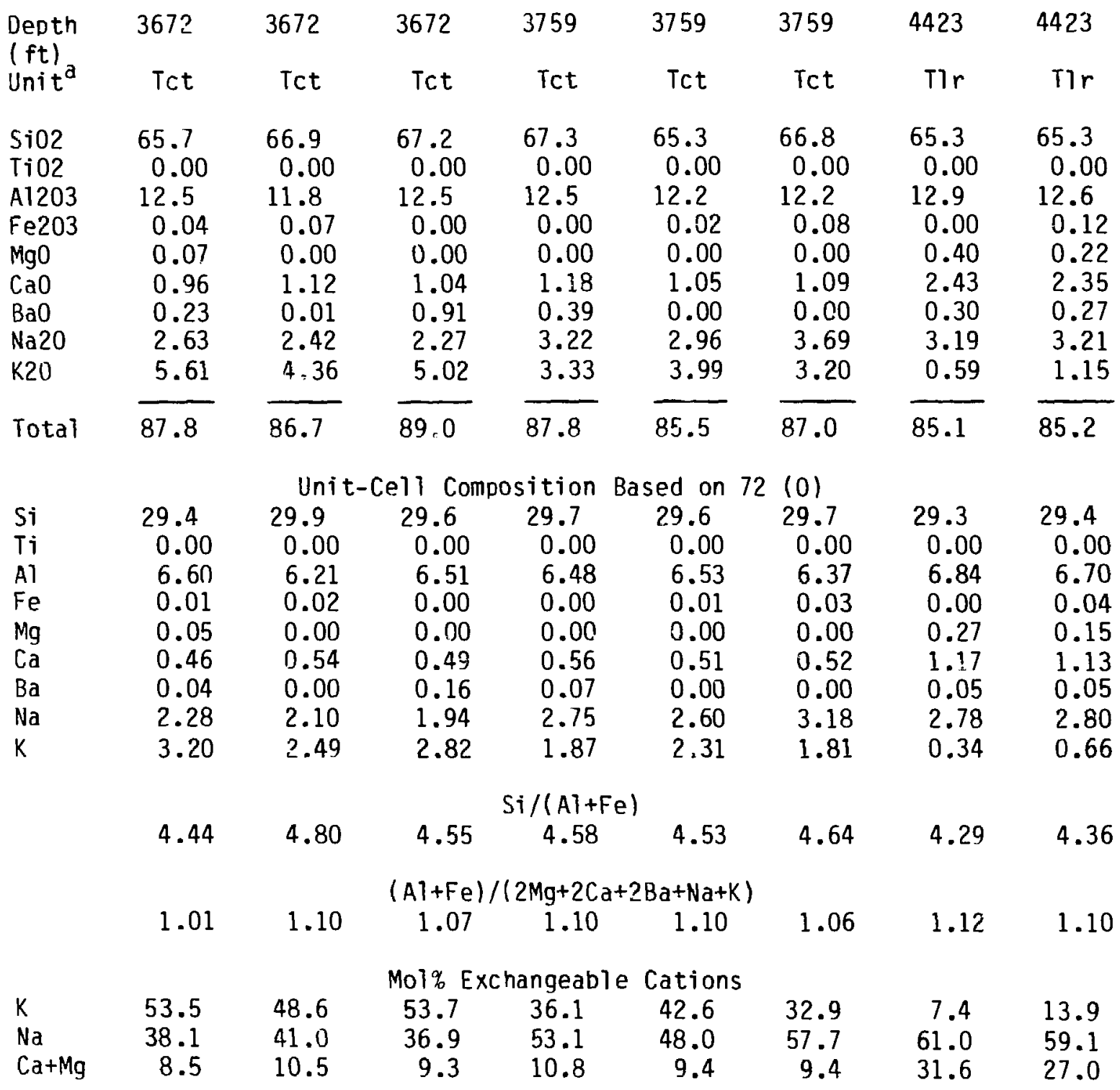




\section{APPENDIX $F$ (cont) \\ Drill Hole USW G-4}

\begin{tabular}{|c|c|c|c|c|c|c|c|c|}
\hline $\begin{array}{l}\text { Deoth } \\
\text { (ft) } \\
\text { Unit }\end{array}$ & 1432 & 1432 & 1470 & 1470 & $\begin{array}{r}1470 \\
\text { Tht }\end{array}$ & $\begin{array}{r}1470 \\
\text { Tht }\end{array}$ & 1470 & 1470 \\
\hline $\begin{array}{l}\mathrm{SiO} 02 \\
\mathrm{TiO} 2 \\
\mathrm{Al} 203 \\
\mathrm{Fe} 203 \\
\mathrm{MgO} \\
\mathrm{CaO} \\
\mathrm{BaO} \\
\mathrm{Na} 20 \\
\mathrm{~K} 20\end{array}$ & $\begin{array}{c}67.1 \\
0.00 \\
12.2 \\
0.00 \\
0.02 \\
1.53 \\
0.18 \\
2.34 \\
5.42\end{array}$ & $\begin{array}{c}67.4 \\
0.00 \\
12.4 \\
0.00 \\
0.00 \\
1.39 \\
1.03 \\
2.42 \\
4.93\end{array}$ & $\begin{array}{c}68.4 \\
0.00 \\
11.8 \\
0.00 \\
0.00 \\
0.84 \\
0.00 \\
2.50 \\
5.43\end{array}$ & $\begin{array}{c}66.0 \\
0.00 \\
12.3 \\
0.00 \\
0.00 \\
0.98 \\
0.00 \\
2.15 \\
5.41\end{array}$ & $\begin{array}{c}67.1 \\
0.00 \\
12.4 \\
0.00 \\
0.00 \\
1.06 \\
0.00 \\
2.55 \\
5.54\end{array}$ & $\begin{array}{c}67.8 \\
0.00 \\
12.0 \\
0.00 \\
0.00 \\
0.89 \\
0.00 \\
2.35 \\
5.68\end{array}$ & $\begin{array}{c}67.4 \\
0.00 \\
11.8 \\
0.00 \\
0.00 \\
0.87 \\
0.08 \\
2.13 \\
5.03\end{array}$ & $\begin{array}{c}67.1 \\
0.00 \\
12.4 \\
0.00 \\
0.00 \\
1.08 \\
0.00 \\
2.24 \\
4.97\end{array}$ \\
\hline Total & 88.8 & 89.5 & 88.9 & 86.8 & 88.6 & 88.7 & 87.3 & 87.8 \\
\hline $\begin{array}{l}\mathrm{Si} \\
\mathrm{Ti} \\
\mathrm{Al} \\
\mathrm{Fe} \\
\mathrm{Mg} \\
\mathrm{Ca} \\
\mathrm{Ba} \\
\mathrm{Na} \\
\mathrm{K}\end{array}$ & $\begin{array}{c}29.6 \\
0.00 \\
6.35 \\
0.00 \\
0.01 \\
0.72 \\
0.03 \\
2.00 \\
3.05\end{array}$ & $\begin{array}{l}\text { Uni } \\
29.6 \\
0.00 \\
6.40 \\
0.00 \\
0.00 \\
0.65 \\
0.18 \\
2.06 \\
2.76\end{array}$ & $\begin{array}{c}\text { Cel1 Co } \\
30.0 \\
0.00 \\
6.08 \\
0.00 \\
0.00 \\
0.39 \\
0.00 \\
2.12 \\
3.03\end{array}$ & $\begin{array}{c}\text { osition } \\
29.6 \\
0.00 \\
6.51 \\
0.00 \\
0.00 \\
0.47 \\
0.00 \\
1.87 \\
3.10\end{array}$ & $\begin{array}{c}\text { Based on } \\
29.6 \\
0.00 \\
6.45 \\
0.00 \\
0.00 \\
0.50 \\
0.00 \\
2.18 \\
3.12\end{array}$ & $\begin{array}{c}72(0) \\
29.8 \\
0.00 \\
6.23 \\
0.00 \\
0.00 \\
0.42 \\
0.00 \\
2.01 \\
3.19\end{array}$ & $\begin{array}{c}30.0 \\
0.00 \\
6.16 \\
0.00 \\
0.00 \\
0.41 \\
0.01 \\
1.84 \\
2.85\end{array}$ & $\begin{array}{c}29.7 \\
0.00 \\
6.49 \\
0.00 \\
0.00 \\
0.51 \\
0.00 \\
1.92 \\
2.81\end{array}$ \\
\hline \multicolumn{9}{|c|}{$\mathrm{Si} /(\mathrm{Al}+\mathrm{Fe})$} \\
\hline & 0.96 & 0.99 & $\begin{array}{c}(A 1+F e) \\
1.02\end{array}$ & $\begin{array}{c}2 \mathrm{Mg}+2 \mathrm{Ca} \\
1.10\end{array}$ & $\begin{array}{c}-2 \mathrm{Ba}+\mathrm{Na}+\mathrm{K} \\
1.02\end{array}$ & 1.03 & 1.11 & 1.13 \\
\hline $\begin{array}{l}\mathrm{K} \\
\mathrm{Na} \\
\mathrm{Ca}+\mathrm{Mg}\end{array}$ & $\begin{array}{l}52.7 \\
34.6 \\
12.7\end{array}$ & $\begin{array}{l}50.4 \\
37.6 \\
11.9\end{array}$ & $\begin{array}{c}\text { Mol\% Ex } \\
54.7 \\
38.2 \\
7.1\end{array}$ & $\begin{array}{c}\text { angeab } \\
56.9 \\
34.4 \\
8.7\end{array}$ & $\begin{array}{c}\text { Cations } \\
53.8 \\
37.6 \\
8.6\end{array}$ & $\begin{array}{r}56.8 \\
35.7 \\
7.5\end{array}$ & $\begin{array}{r}55.9 \\
36.0 \\
8.1\end{array}$ & $\begin{array}{r}53.5 \\
36.7 \\
9.8\end{array}$ \\
\hline
\end{tabular}




\section{APPENDIX F (cont)}

Drill Hole USW G-4

\begin{tabular}{|c|c|c|c|c|c|c|c|c|}
\hline $\begin{array}{l}\text { Depth } \\
(\mathrm{ft})\end{array}$ & 1544 & 1544 & 1544 & 1544 & 1544 & 1707 & 1707 & 1707 \\
\hline Unit ${ }^{a}$ & Tht & Tht & Tht & Tht & Tht & Tht & Trit & Tht \\
\hline SiO2 & 64.1 & 66.0 & 65.5 & 64.5 & 68.9 & 67.3 & 55.9 & 67.2 \\
\hline Ti02 & 0.00 & 0.00 & 0.01 & 0.02 & 0.02 & 0.00 & 0.00 & 0.00 \\
\hline Al203 & 12.7 & 12.8 & 12.5 & 12.0 & 13.0 & 12.8 & 13.1 & 12.3 \\
\hline $\mathrm{Fe} 203$ & 0.05 & 0.11 & 0.13 & 0.09 & 0.12 & 0.00 & 0.03 & 0.00 \\
\hline Mg0 & 0.00 & 0.00 & i). .02 & 0.00 & 0.02 & 0.01 & 0.05 & 0.02 \\
\hline $\mathrm{CaO}$ & 0.94 & 1.09 & 0.95 & 0.79 & 0.81 & 0.94 & 1.09 & 0.93 \\
\hline $\mathrm{BaO}$ & 0.09 & 0.06 & 0.11 & 0.05 & 0.10 & 0.02 & 0.10 & 0.05 \\
\hline $\mathrm{Na} 20$ & 2.88 & 3.01 & 3.08 & 3.10 & 3.25 & 2.67 & 2.67 & 2.61 \\
\hline K20 & 5.36 & 5.26 & 5.45 & 5.66 & 5.62 & 5.45 & 5.72 & 6.28 \\
\hline Total & 86.1 & 88.3 & 87.8 & 86.3 & 91.8 & 89.2 & 88.6 & 89.4 \\
\hline
\end{tabular}

Unit-Cell Composition Based on $72(0)$

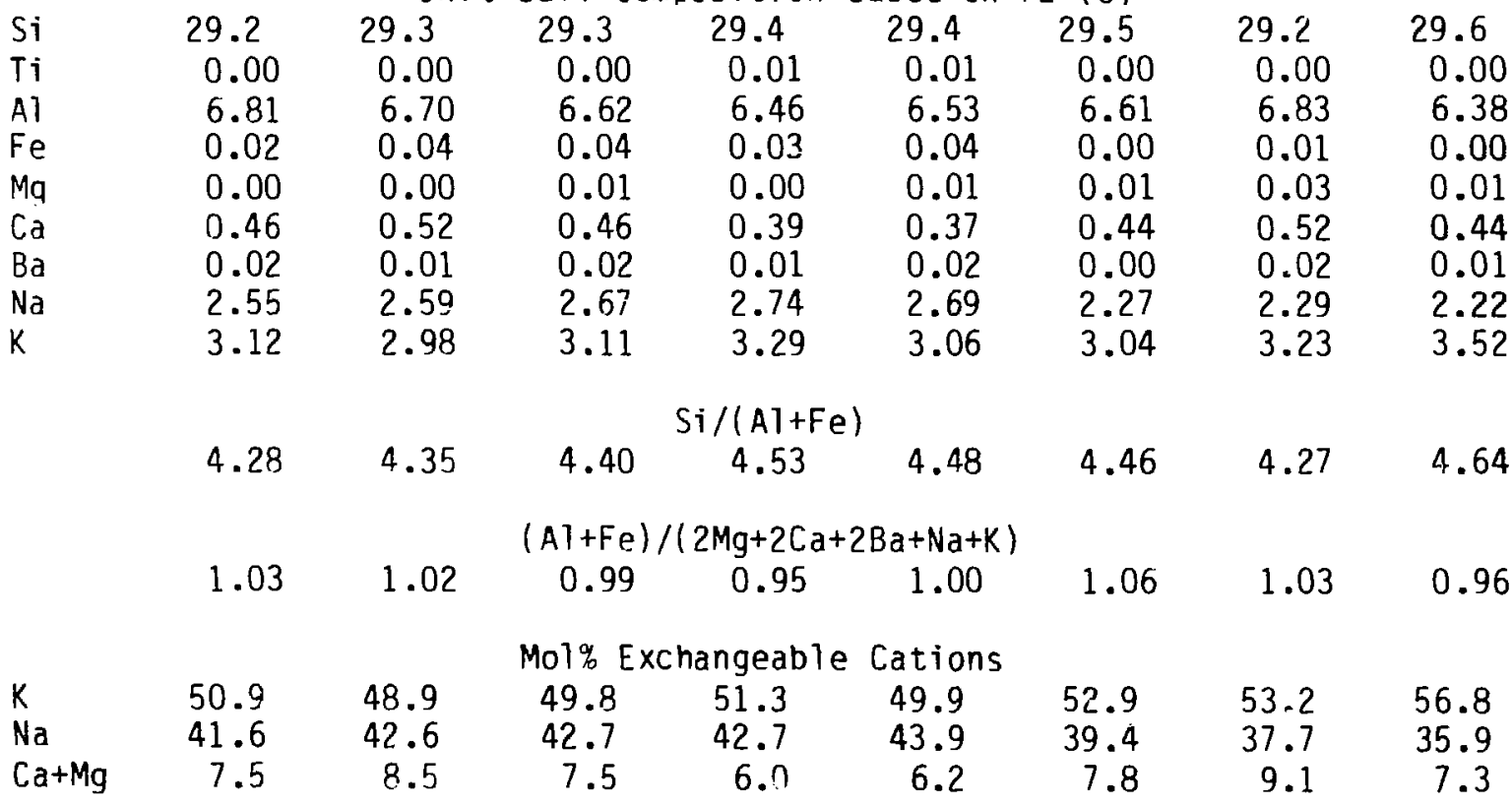


APPENDIX $F$ (cont)

Drill Hole USW G-4

\begin{tabular}{|c|c|c|c|c|c|c|c|c|}
\hline $\begin{array}{l}\text { Deoth } \\
\text { (ft) } \\
\text { Unit }^{\mathrm{a}}\end{array}$ & 1707 & 1707 & $17 \delta 2$ & 1762 & 1762 & 1762 & 1762 & 1762 \\
\hline $\begin{array}{l}\mathrm{Si02} \\
\mathrm{Ti02} \\
\mathrm{Al} 203 \\
\mathrm{Fe} 203 \\
\mathrm{Mg0} \\
\mathrm{Ca0} \\
\mathrm{Ba0} \\
\mathrm{Na} 20 \\
\mathrm{~K} 20\end{array}$ & $\begin{array}{c}64.4 \\
0.00 \\
12.7 \\
0.00 \\
0.05 \\
0.84 \\
0.05 \\
1.91 \\
6.59\end{array}$ & $\begin{array}{c}68.1 \\
0.00 \\
12.6 \\
0.00 \\
0.06 \\
1.05 \\
0.04 \\
2.42 \\
5.78\end{array}$ & $\begin{array}{c}69.3 \\
0.00 \\
12.9 \\
0.00 \\
0.07 \\
1.74 \\
0.00 \\
3.46 \\
3.86\end{array}$ & $\begin{array}{c}68.0 \\
0.00 \\
13.0 \\
0.00 \\
0.09 \\
1.60 \\
0.00 \\
2.71 \\
3.67\end{array}$ & $\begin{array}{c}68.8 \\
0.00 \\
13.4 \\
0.00 \\
0.08 \\
1.65 \\
0.21 \\
3.17 \\
3.87\end{array}$ & $\begin{array}{c}69.5 \\
0.00 \\
13.3 \\
0.04 \\
0.09 \\
1.73 \\
0.10 \\
3.33 \\
3.74\end{array}$ & $\begin{array}{c}67.0 \\
0.01 \\
13.4 \\
0.05 \\
0.12 \\
1.79 \\
0.16 \\
3.45 \\
3.77\end{array}$ & $\begin{array}{c}57.3 \\
0.02 \\
13.0 \\
0.00 \\
0.15 \\
1.65 \\
0.18 \\
3.19 \\
3.90\end{array}$ \\
\hline Total & 86.6 & 90.0 & 91.3 & 89.0 & 91.2 & 91.8 & 89.7 & 89.4 \\
\hline $\begin{array}{l}\mathrm{Si} \\
\mathrm{Ti} \\
\mathrm{Al} \\
\mathrm{Fe} \\
\mathrm{Mg} \\
\mathrm{Ca} \\
\mathrm{Ba} \\
\mathrm{Na} \\
\mathrm{K}\end{array}$ & $\begin{array}{c}29.3 \\
0.00 \\
6.83 \\
0.00 \\
0.03 \\
0.41 \\
0.01 \\
1.68 \\
3.82\end{array}$ & $\begin{array}{l}\text { Uni } \\
29.6 \\
0.00 \\
6.46 \\
0.00 \\
0.04 \\
0.49 \\
0.01 \\
2.04 \\
3.20\end{array}$ & $\begin{array}{c}\text { Ce11 Co } \\
29.5 \\
0.00 \\
6.47 \\
0.00 \\
0.04 \\
0.79 \\
0.00 \\
2.86 \\
2.10\end{array}$ & $\begin{array}{c}\text { osition } \\
29.5 \\
0.00 \\
6.65 \\
0.00 \\
0.06 \\
0.75 \\
0.00 \\
2.28 \\
2.03\end{array}$ & $\begin{array}{c}\text { Based on } \\
29.4 \\
0.00 \\
6.72 \\
0.00 \\
0.05 \\
0.75 \\
0.04 \\
2.62 \\
2.11\end{array}$ & $\begin{array}{c}72(0) \\
29.4 \\
0.00 \\
6.62 \\
0.01 \\
0.06 \\
0.78 \\
0.02 \\
2.73 \\
2.02\end{array}$ & $\begin{array}{c}29.1 \\
0.00 \\
6.85 \\
0.02 \\
0.08 \\
0.83 \\
0.03 \\
2.91 \\
2.09\end{array}$ & $\begin{array}{c}29.3 \\
0.01 \\
6.67 \\
0.00 \\
0.10 \\
0.77 \\
0.03 \\
2.70 \\
2.17\end{array}$ \\
\hline \multicolumn{9}{|c|}{$\mathrm{Si} /(\mathrm{Al}+\mathrm{Fe})$} \\
\hline \multicolumn{9}{|c|}{$(\mathrm{A} 1+\mathrm{Fe}) /(2 \mathrm{Mg}+2 \mathrm{Ca}+2 \mathrm{Ba}+\mathrm{Na}+\mathrm{K})$} \\
\hline $\begin{array}{l}\mathrm{K} \\
\mathrm{Na} \\
\mathrm{Ca}+\mathrm{Mg}\end{array}$ & $\begin{array}{r}64.3 \\
28.3 \\
7.4\end{array}$ & $\begin{array}{r}55.5 \\
35.3 \\
9.1\end{array}$ & $\begin{array}{l}\text { Mol\% Ex } \\
36.2 \\
49.3 \\
14.5\end{array}$ & $\begin{array}{l}\text { angeabl } \\
39.7 \\
44.6 \\
15.7\end{array}$ & $\begin{array}{c}\text { Cations } \\
38.1 \\
47.4 \\
14.5\end{array}$ & $\begin{array}{l}36.1 \\
48.9 \\
15.0\end{array}$ & $\begin{array}{l}35.4 \\
49.2 \\
15.4\end{array}$ & $\begin{array}{l}37.8 \\
47.0 \\
15.1\end{array}$ \\
\hline
\end{tabular}


APPENDIX F (cont;

Drill Hole USW G-4

\begin{tabular}{|c|c|c|c|c|c|c|c|c|}
\hline $\begin{array}{l}\text { Oepth } \\
(\mathrm{ft})^{\mathrm{a}} \\
\text { Unit }\end{array}$ & 1762 & 2132 & 2132 & 2132 & 2132 & 2132 & 2132 & 2132 \\
\hline $\begin{array}{l}\mathrm{SiO} 2 \\
\mathrm{TiO} 02 \\
\mathrm{~A} 1203 \\
\mathrm{Fe} 203 \\
\mathrm{HgO} \\
\mathrm{CaO} \\
\mathrm{BaO} \\
\mathrm{Na2O} \\
\mathrm{K} 2 \mathrm{O}\end{array}$ & $\begin{array}{c}66.0 \\
0.02 \\
13.2 \\
0.04 \\
0.09 \\
1.70 \\
0.24 \\
3.08 \\
4.29\end{array}$ & $\begin{array}{c}64.5 \\
0.00 \\
11.8 \\
0.00 \\
0.05 \\
3.45 \\
0.11 \\
1.02 \\
2.14\end{array}$ & $\begin{array}{c}65.5 \\
0.00 \\
11.7 \\
0.00 \\
0.04 \\
3.44 \\
0.00 \\
0.93 \\
2.36\end{array}$ & $\begin{array}{c}65.0 \\
0.00 \\
11.1 \\
0.07 \\
0.06 \\
3.50 \\
0.00 \\
1.35 \\
2.21\end{array}$ & $\begin{array}{c}65.6 \\
0.00 \\
12.1 \\
0.04 \\
0.05 \\
3.42 \\
0.16 \\
1.07 \\
2.31\end{array}$ & $\begin{array}{c}65.1 \\
0.00 \\
11.8 \\
0.00 \\
0.05 \\
3.58 \\
0.03 \\
0.92 \\
2.30\end{array}$ & $\begin{array}{c}65.1 \\
0.00 \\
12.3 \\
0.01 \\
0.03 \\
3.59 \\
0.00 \\
1.18 \\
1.69\end{array}$ & $\begin{array}{c}66.3 \\
0.00 \\
11.6 \\
0.00 \\
0.03 \\
3.63 \\
0.00 \\
1.11 \\
1.98\end{array}$ \\
\hline Total & 88.7 & 83.0 & 84.0 & 83.2 & 84.8 & 83.8 & 84.0 & 84.7 \\
\hline $\begin{array}{l}\mathrm{Si} \\
\mathrm{Ti} \\
\mathrm{Al} \\
\mathrm{Fe} \\
\mathrm{Mg} \\
\mathrm{Ca} \\
\mathrm{Ba} \\
\mathrm{Na} \\
\mathrm{K}\end{array}$ & $\begin{array}{c}29.1 \\
0.01 \\
6.88 \\
0.01 \\
0.06 \\
0.80 \\
0.04 \\
2.63 \\
2.41\end{array}$ & $\begin{array}{l}\text { Uni } \\
29.8 \\
0.00 \\
6.40 \\
0.00 \\
0.03 \\
1.71 \\
0.02 \\
0.91 \\
1.26\end{array}$ & $\begin{array}{c}\text { Ce11 Co } \\
29.9 \\
0.00 \\
6.28 \\
0.00 \\
0.03 \\
1.68 \\
0.00 \\
0.82 \\
1.37\end{array}$ & $\begin{array}{c}\text { ositior } \\
30.0 \\
0.00 \\
6.01 \\
0.02 \\
0.04 \\
1.73 \\
0.00 \\
1.21 \\
1.30\end{array}$ & $\begin{array}{c}\text { ased or } \\
29.7 \\
0.00 \\
6.47 \\
0.01 \\
0.03 \\
1.66 \\
0.03 \\
0.94 \\
1.33\end{array}$ & $\begin{array}{c}2(0) \\
29.8 \\
0.00 \\
6.38 \\
0.00 \\
0.03 \\
1.75 \\
0.01 \\
0.82 \\
1.34\end{array}$ & $\begin{array}{c}29.6 \\
0.00 \\
6.62 \\
0.00 \\
0.02 \\
1.75 \\
0.00 \\
1.04 \\
0.98\end{array}$ & $\begin{array}{l}29.9 \\
0.00 \\
6.20 \\
0.00 \\
0.02 \\
1.76 \\
0.00 \\
0.97 \\
1.14\end{array}$ \\
\hline \multicolumn{9}{|c|}{$\mathrm{Si} /(\mathrm{Al}+\mathrm{Fe})$} \\
\hline \multicolumn{9}{|c|}{$(\mathrm{A} l+\mathrm{Fe}) /(2 \mathrm{Mg}+2 \mathrm{Ca}+2 \mathrm{Ba}+\mathrm{Na}+\mathrm{K})$} \\
\hline $\begin{array}{l}\mathrm{K} \\
\mathrm{Na} \\
\mathrm{Ca}+\mathrm{Mg}\end{array}$ & $\begin{array}{l}40.8 \\
44.6 \\
14.6\end{array}$ & $\begin{array}{l}32.2 \\
23.3 \\
44.5\end{array}$ & $\begin{array}{c}\text { Mol\% E } \\
35.2 \\
21.1 \\
43.8\end{array}$ & $\begin{array}{l}\text { angeab } \\
30.4 \\
28.2 \\
41.4\end{array}$ & $\begin{array}{c}\text { Cation } \\
33.6 \\
23.7 \\
42.7\end{array}$ & $\begin{array}{l}34.0 \\
20.7 \\
45.3\end{array}$ & $\begin{array}{l}25.9 \\
27.4 \\
46.7\end{array}$ & $\begin{array}{l}29.3 \\
25.0 \\
45.7\end{array}$ \\
\hline
\end{tabular}


APPENDIX F (cont)

Drill Hole USW G-4

\begin{tabular}{|c|c|c|c|c|c|c|c|c|}
\hline $\begin{array}{l}\text { Depth } \\
(\mathrm{ft}) \\
\text { Unit }^{\mathrm{a}}\end{array}$ & 2132 & 2132 & 2132 & 2227 & 2227 & 2227 & 2227 & 2227 \\
\hline $\begin{array}{l}\mathrm{SiO} 2 \\
\mathrm{TiO} 2 \\
\mathrm{Al} 203 \\
\mathrm{Fe} 203 \\
\mathrm{MgO} \\
\mathrm{CaO} \\
\mathrm{BaO} \\
\mathrm{Na} 20 \\
\mathrm{~K} 20\end{array}$ & $\begin{array}{c}65.1 \\
0.00 \\
11.1 \\
0.04 \\
0.04 \\
3.59 \\
0.00 \\
0.97 \\
1.93\end{array}$ & $\begin{array}{c}65.4 \\
0.00 \\
12.0 \\
0.01 \\
0.02 \\
3.48 \\
0.00 \\
1.18 \\
2.05\end{array}$ & $\begin{array}{c}64.3 \\
0.00 \\
12.1 \\
0.06 \\
0.03 \\
3.78 \\
0.00 \\
1.33 \\
1.78\end{array}$ & $\begin{array}{c}64.0 \\
0.00 \\
12.1 \\
0.02 \\
0.11 \\
3.69 \\
0.00 \\
1.22 \\
1.42\end{array}$ & $\begin{array}{c}65.8 \\
0.00 \\
12.4 \\
0.00 \\
0.14 \\
3.92 \\
0.00 \\
0.84 \\
1.62\end{array}$ & $\begin{array}{c}64.1 \\
0.00 \\
12.1 \\
0.04 \\
0.11 \\
3.88 \\
0.02 \\
1.21 \\
1.14\end{array}$ & $\begin{array}{c}64.7 \\
0.00 \\
12.2 \\
0.02 \\
0.19 \\
4.02 \\
0.00 \\
1.15 \\
1.67\end{array}$ & $\begin{array}{c}65.8 \\
0.00 \\
12.3 \\
0.03 \\
0.10 \\
3.51 \\
0.00 \\
1.38 \\
1.85\end{array}$ \\
\hline Total & 82.8 & 84.2 & 83.4 & 82.5 & 84.6 & 82.6 & 84.0 & 84.9 \\
\hline $\begin{array}{l}\mathrm{Si} \\
\mathrm{Ti} \\
\mathrm{Al} \\
\mathrm{Fe} \\
\mathrm{Mg} \\
\mathrm{Ca} \\
\mathrm{Ba} \\
\mathrm{Na} \\
\mathrm{K}\end{array}$ & $\begin{array}{c}30.0 \\
0.00 \\
6.05 \\
0.01 \\
0.03 \\
1.77 \\
0.00 \\
0.87 \\
1.14\end{array}$ & $\begin{array}{c}\text { Uni } \\
29.7 \\
0.00 \\
6.45 \\
0.00 \\
0.01 \\
1.70 \\
0.00 \\
1.04 \\
1.19\end{array}$ & $\begin{array}{c}\text { Ce11 Co } \\
29.6 \\
0.00 \\
6.55 \\
0.02 \\
0.02 \\
1.86 \\
0.00 \\
1.19 \\
1.04\end{array}$ & $\begin{array}{c}\text { osition } \\
29.6 \\
0.00 \\
6.58 \\
0.01 \\
0.08 \\
1.83 \\
0.00 \\
1.09 \\
0.84\end{array}$ & $\begin{array}{c}\text { Based on } \\
29.7 \\
0.00 \\
6.57 \\
0.00 \\
0.09 \\
1.80 \\
0.00 \\
0.73 \\
0.93\end{array}$ & $\begin{array}{c}72(0) \\
29.6 \\
0.00 \\
6.59 \\
0.01 \\
0.08 \\
1.92 \\
0.00 \\
1.08 \\
0.67\end{array}$ & $\begin{array}{l}29.5 \\
0.00 \\
6.57 \\
0.01 \\
0.13 \\
1.96 \\
0.00 \\
1.02 \\
0.97\end{array}$ & $\begin{array}{l}29.7 \\
0.00 \\
6.51 \\
0.01 \\
0.07 \\
1.70 \\
0.00 \\
1.21 \\
1.06\end{array}$ \\
\hline \multicolumn{9}{|c|}{$\mathrm{Si} /(\mathrm{A} 1+\mathrm{Fe})$} \\
\hline \multicolumn{9}{|c|}{$(\mathrm{A} 1+\mathrm{Fe}) /(2 \mathrm{Mg}+2 \mathrm{Ca}+2 \mathrm{Ba}+\mathrm{Na}+\mathrm{K})$} \\
\hline $\begin{array}{l}\mathrm{K} \\
\mathrm{Na} \\
\mathrm{Ca}+\mathrm{Mg}\end{array}$ & $\begin{array}{l}29.8 \\
22.8 \\
47.4\end{array}$ & $\begin{array}{l}30.2 \\
26.4 \\
43.4\end{array}$ & $\begin{array}{c}\text { Mol\% Ex } \\
25.4 \\
28.8 \\
45.8\end{array}$ & $\begin{array}{c}\text { angeabl } \\
21.9 \\
28.5 \\
49.6\end{array}$ & $\begin{array}{c}\text { Cations } \\
25.5 \\
20.1 \\
54.4\end{array}$ & $\begin{array}{l}17.9 \\
28.9 \\
53.2\end{array}$ & $\begin{array}{l}23.8 \\
24.9 \\
51.3\end{array}$ & $\begin{array}{l}26.4 \\
29.9 \\
43.7\end{array}$ \\
\hline
\end{tabular}




\section{APPENDIX $F($ cont $)$}

Drill Hole USW G-4

\begin{tabular}{|c|c|c|c|c|c|c|c|c|}
\hline $\begin{array}{l}\text { Depth } \\
\text { (ft) } \\
\text { Unit }\end{array}$ & 2227 & 2227 & 2239 & 2239 & 2239 & 2239 & 2239 & 2239 \\
\hline $\begin{array}{l}\mathrm{SiO} 2 \\
\mathrm{TiO} 02 \\
\mathrm{Al} 203 \\
\mathrm{Fe} 203 \\
\mathrm{MgO} \\
\mathrm{CaO} \\
\mathrm{BaO} \\
\mathrm{Na2O} \\
\mathrm{K} 20\end{array}$ & $\begin{array}{c}65.5 \\
0.00 \\
11.7 \\
0.10 \\
0.19 \\
3.72 \\
0.16 \\
0.80 \\
1.33\end{array}$ & $\begin{array}{c}67.1 \\
0.00 \\
11.6 \\
0.01 \\
0.13 \\
3.38 \\
0.03 \\
1.02 \\
1.84\end{array}$ & $\begin{array}{c}66.3 \\
0.00 \\
12.0 \\
0.01 \\
0.37 \\
4.02 \\
0.29 \\
0.96 \\
1.43\end{array}$ & $\begin{array}{c}64.6 \\
0.00 \\
12.1 \\
0.05 \\
0.31 \\
4.09 \\
0.03 \\
0.52 \\
1.11\end{array}$ & $\begin{array}{c}65.7 \\
0.01 \\
12.2 \\
0.06 \\
0.37 \\
4.01 \\
0.10 \\
0.83 \\
1.68\end{array}$ & $\begin{array}{c}66.9 \\
0.02 \\
12.1 \\
0.12 \\
0.45 \\
3.89 \\
0.06 \\
0.39 \\
1.49\end{array}$ & $\begin{array}{c}65.4 \\
0.00 \\
10.9 \\
0.08 \\
0.45 \\
3.65 \\
0.00 \\
0.80 \\
1.91\end{array}$ & $\begin{array}{c}65.2 \\
0.00 \\
12.0 \\
0.09 \\
0.37 \\
3.97 \\
0.04 \\
0.50 \\
1.29\end{array}$ \\
\hline Total & 83.5 & 85.1 & 85.3 & 82.8 & 85.0 & 85.5 & 83.2 & 83.4 \\
\hline $\begin{array}{l}\mathrm{Si} \\
\mathrm{Ti} \\
\mathrm{Al} \\
\mathrm{Fe} \\
\mathrm{Mg} \\
\mathrm{Ca} \\
\mathrm{Ba} \\
\mathrm{Na} \\
\mathrm{K}\end{array}$ & $\begin{array}{l}29.9 \\
0.00 \\
6.31 \\
0.03 \\
0.13 \\
1.82 \\
0.03 \\
0.71 \\
0.77\end{array}$ & $\begin{array}{c}\text { Uni } \\
30.1 \\
0.00 \\
6.13 \\
0.00 \\
0.09 \\
1.62 \\
0.01 \\
0.89 \\
1.05\end{array}$ & $\begin{array}{c}\text { Cel1 Co } \\
29.7 \\
0.00 \\
6.34 \\
0.00 \\
0.25 \\
1.93 \\
0.05 \\
0.83 \\
0.82\end{array}$ & $\begin{array}{l}\text { ositior } \\
29.7 \\
0.00 \\
6.53 \\
0.02 \\
0.21 \\
2.01 \\
0.01 \\
0.46 \\
0.65\end{array}$ & $\begin{array}{c}\text { 3ased on } \\
29.6 \\
0.00 \\
6.49 \\
0.02 \\
0.25 \\
1.94 \\
0.02 \\
0.73 \\
0.97\end{array}$ & $\begin{array}{c}2(0) \\
29.8 \\
0.01 \\
6.36 \\
0.04 \\
0.30 \\
1.86 \\
0.01 \\
0.34 \\
0.85\end{array}$ & $\begin{array}{c}30.0 \\
0.00 \\
5.93 \\
0.03 \\
0.31 \\
1.80 \\
0.00 \\
0.71 \\
1.12\end{array}$ & $\begin{array}{l}29.8 \\
0.00 \\
6.43 \\
0.03 \\
0.25 \\
1.94 \\
0.01 \\
0.44 \\
0.75\end{array}$ \\
\hline & 1.17 & 1.14 & $\begin{array}{r}4.68 \\
(\mathrm{~A} 1+\mathrm{Fe}) \\
1.04\end{array}$ & $\begin{array}{r}2 \mathrm{Mg}+2 \mathrm{C} \\
1.17\end{array}$ & $\begin{array}{c}4.55 \\
B a+N a+K \\
1.07\end{array}$ & 4.66 & 5.04 & 4.60 \\
\hline $\begin{array}{l}\mathrm{K} \\
\mathrm{Na} \\
\mathrm{Ca}+\mathrm{Mg}\end{array}$ & $\begin{array}{l}22.6 \\
20.6 \\
56.8\end{array}$ & $\begin{array}{l}28.8 \\
24.3 \\
46.9\end{array}$ & $\begin{array}{c}\text { Mol\% E) } \\
21.4 \\
21.8 \\
56.9\end{array}$ & $\begin{array}{l}\text { angeab } \\
19.5 \\
13.9 \\
66.6\end{array}$ & $\begin{array}{c}\text { Cations } \\
24.9 \\
18.7 \\
56.4\end{array}$ & $\begin{array}{l}25.4 \\
10.1 \\
64.6\end{array}$ & $\begin{array}{l}28.4 \\
18.1 \\
53.5\end{array}$ & $\begin{array}{l}22.2 \\
13.1 \\
64.8\end{array}$ \\
\hline
\end{tabular}




\section{APPENDIX $F$ (cont)}

Drill Hole USW G-4

\begin{tabular}{|c|c|c|c|c|c|c|c|c|}
\hline $\begin{array}{l}\text { Depth } \\
\text { (ft) } \\
\text { Unit }\end{array}$ & 2239 & $\begin{array}{r}2738 \\
\text { Tcb }\end{array}$ & $\begin{array}{r}2738 \\
\text { Tcb }\end{array}$ & $\begin{array}{r}2738 \\
\text { Tcb }\end{array}$ & $\begin{array}{r}2738 \\
\text { Tcb }\end{array}$ & $\begin{array}{r}2738 \\
\text { Tcb }\end{array}$ & $\begin{array}{r}2738 \\
\text { Tcb }\end{array}$ & $\begin{array}{r}2738 \\
\text { Tcb }\end{array}$ \\
\hline $\begin{array}{l}\mathrm{SiO} 02 \\
\mathrm{Ti02} \\
\mathrm{Al} 203 \\
\mathrm{Fe} 203 \\
\mathrm{MgO} \\
\mathrm{CaO} \\
\mathrm{BaO} \\
\mathrm{Na} 20 \\
\mathrm{~K} 20\end{array}$ & $\begin{array}{c}66.6 \\
0.00 \\
11.9 \\
0.07 \\
0.37 \\
3.59 \\
0.00 \\
0.63 \\
1.71\end{array}$ & $\begin{array}{c}65.9 \\
0.00 \\
11.8 \\
0.00 \\
0.29 \\
4.32 \\
0.04 \\
0.80 \\
0.91\end{array}$ & $\begin{array}{c}64.9 \\
0.00 \\
12.6 \\
0.00 \\
0.30 \\
4.34 \\
0.20 \\
0.56 \\
1.10\end{array}$ & $\begin{array}{c}66.5 \\
0.00 \\
12.8 \\
0.00 \\
0.37 \\
4.49 \\
0.03 \\
0.87 \\
1.06\end{array}$ & $\begin{array}{c}65.8 \\
0.00 \\
12.4 \\
0.02 \\
0.24 \\
4.26 \\
0.00 \\
0.83 \\
1.02\end{array}$ & $\begin{array}{c}64.9 \\
0.00 \\
11.6 \\
0.00 \\
0.26 \\
4.14 \\
0.14 \\
1.10 \\
1.80\end{array}$ & $\begin{array}{c}66.9 \\
0.00 \\
12.4 \\
0.05 \\
0.27 \\
4.31 \\
0.17 \\
0.57 \\
1.17\end{array}$ & $\begin{array}{c}64.9 \\
0.00 \\
10.6 \\
0.00 \\
0.29 \\
4.15 \\
0.10 \\
0.58 \\
0.63\end{array}$ \\
\hline Total & 84.9 & 84.1 & 84.1 & 86.1 & 84.5 & 84.0 & 85.9 & 81.3 \\
\hline $\begin{array}{l}\mathrm{Si} \\
\mathrm{Ti} \\
\mathrm{Al} \\
\mathrm{Fe} \\
\mathrm{Mg} \\
\mathrm{Ca} \\
\mathrm{Ba} \\
\mathrm{Na} \\
\mathrm{K}\end{array}$ & $\begin{array}{c}29.9 \\
0.00 \\
6.32 \\
0.02 \\
0.25 \\
1.73 \\
0.00 \\
0.55 \\
0.98\end{array}$ & $\begin{array}{c}\text { Uni } \\
29.8 \\
0.00 \\
6.30 \\
0.00 \\
0.20 \\
2.09 \\
0.01 \\
0.70 \\
0.52\end{array}$ & $\begin{array}{c}\text { Cel1 Cc } 29.5 \\
0.00 \\
6.77 \\
0.00 \\
0.20 \\
2.11 \\
0.04 \\
0.49 \\
0.64\end{array}$ & $\begin{array}{c}\text { osition } \\
29.5 \\
0.00 \\
6.66 \\
0.00 \\
0.24 \\
2.13 \\
0.01 \\
0.75 \\
0.60\end{array}$ & $\begin{array}{c}\text { Based on } \\
29.6 \\
0.00 \\
6.57 \\
0.01 \\
0.16 \\
2.06 \\
0.00 \\
0.72 \\
0.59\end{array}$ & $\begin{array}{c}2(0) \\
29.7 \\
0.00 \\
6.26 \\
0.00 \\
0.18 \\
2.03 \\
0.03 \\
0.98 \\
1.05\end{array}$ & $\begin{array}{c}29.7 \\
0.00 \\
5.49 \\
0.02 \\
0.18 \\
2.05 \\
0.03 \\
0.49 \\
0.66\end{array}$ & $\begin{array}{c}30.2 \\
0.00 \\
5.85 \\
0.00 \\
0.20 \\
2.07 \\
0.02 \\
0.52 \\
0.37\end{array}$ \\
\hline \multicolumn{9}{|c|}{$\mathrm{Si} /(\mathrm{Al}+\mathrm{Fe})$} \\
\hline \multicolumn{9}{|c|}{$(A)+F e) /(2 M g+2 C a+2 B a+N a+K)$} \\
\hline $\begin{array}{l}\mathrm{K} \\
\mathrm{Na} \\
\mathrm{Ca}+\mathrm{Mg}\end{array}$ & $\begin{array}{l}28.0 \\
15.7 \\
56.4\end{array}$ & $\begin{array}{l}14.9 \\
20.0 \\
65.1\end{array}$ & $\begin{array}{c}\text { Mol\% E } \\
18.5 \\
14.3 \\
67.2\end{array}$ & $\begin{array}{c}\text { angeab } \\
16.1 \\
20.1 \\
63.8\end{array}$ & $\begin{array}{c}\text { Cation } \\
16.6 \\
20.5 \\
62.8\end{array}$ & $\begin{array}{l}24.8 \\
23.1 \\
52.1\end{array}$ & $\begin{array}{l}19.6 \\
14.5 \\
65.9\end{array}$ & $\begin{array}{l}11.8 \\
16.5 \\
71.7\end{array}$ \\
\hline
\end{tabular}




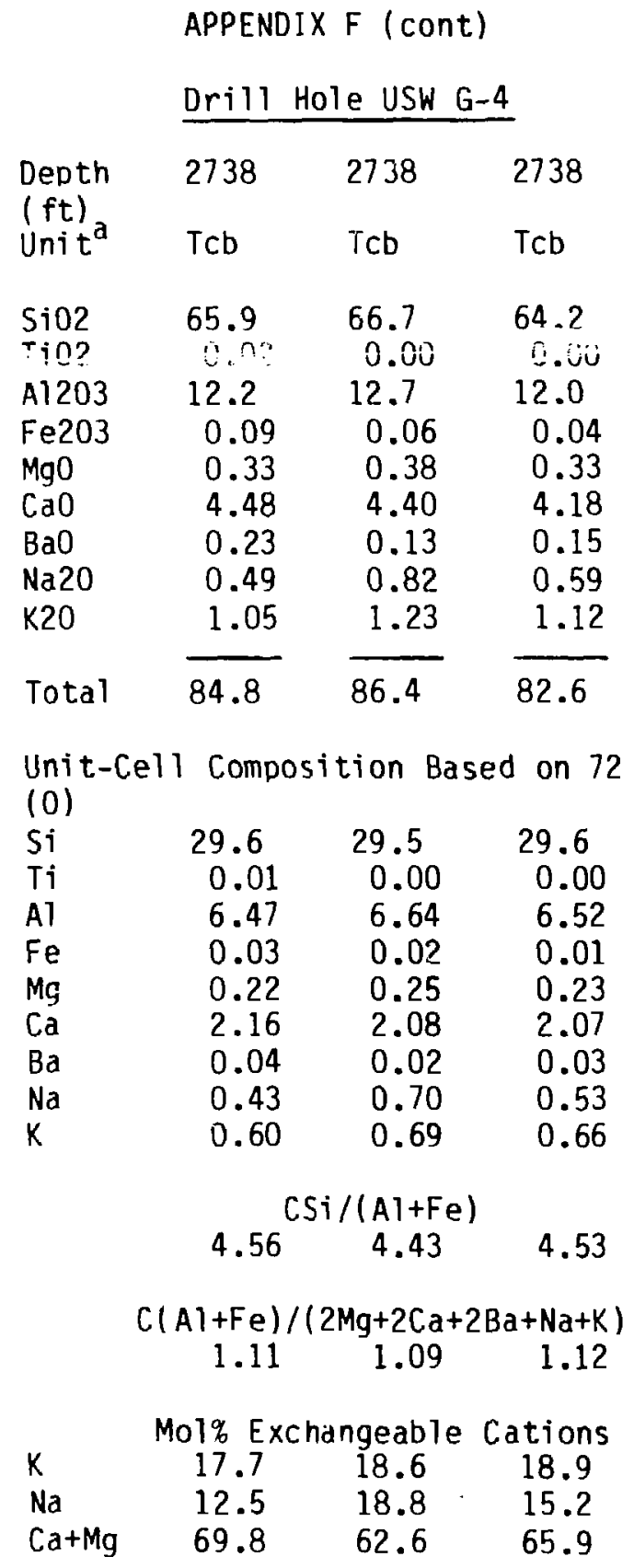




\section{APPENDIX $F$ (cont)}

Drill Hole USW H-3

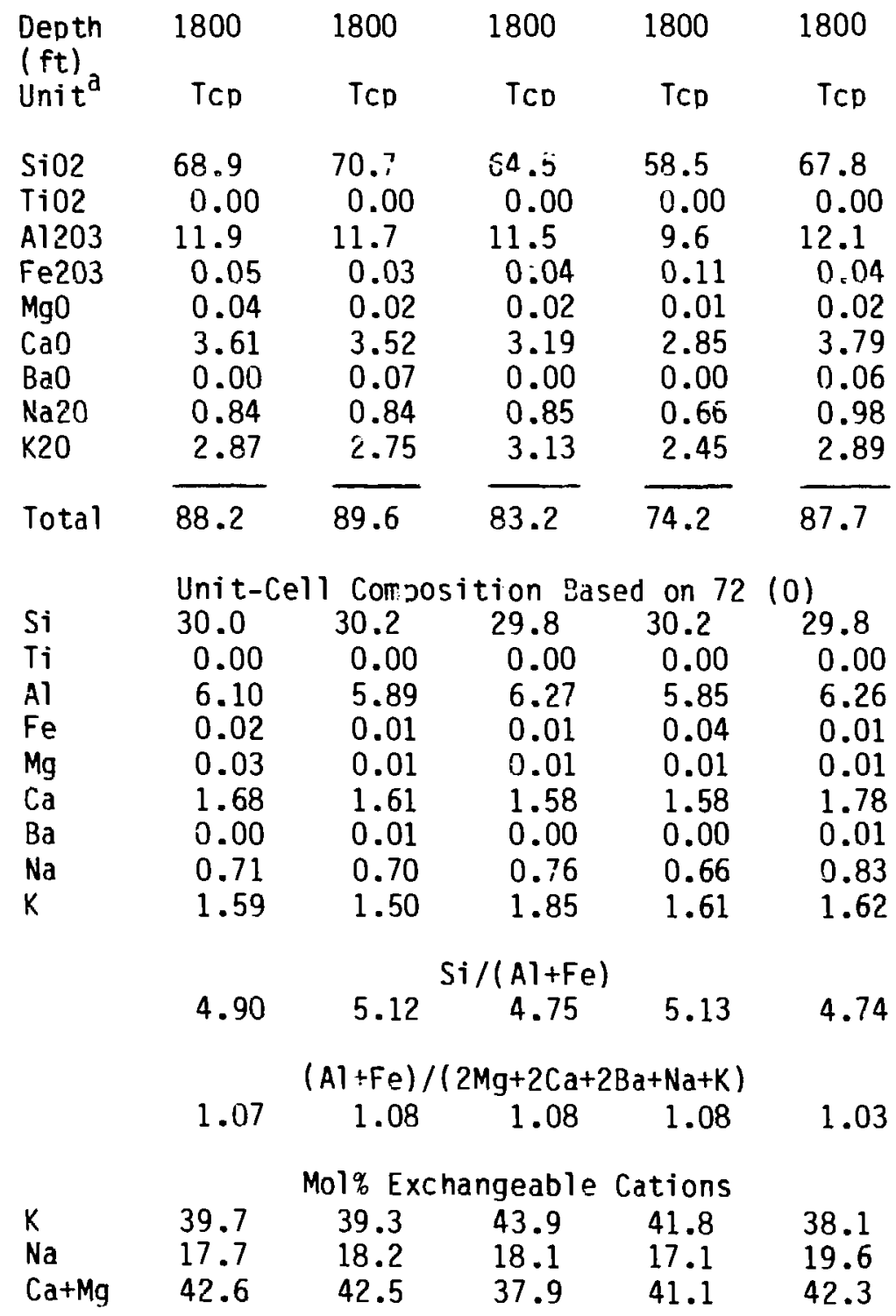




\section{APPENDIX $F$ (cont)}

\section{Drill Hole USW H-4}

\begin{tabular}{|c|c|c|c|c|c|c|c|c|}
\hline $\begin{array}{l}\text { Depth } \\
\text { (ft) } \\
\text { Unit }\end{array}$ & 1420 & 1420 & 1420 & 1420 & 1420 & 1420 & 1455 & 1455 \\
\hline $\begin{array}{l}\mathrm{SiO} 02 \\
\mathrm{Ti02} \\
\mathrm{Al} 203 \\
\mathrm{Fe} 203 \\
\mathrm{MgO} \\
\mathrm{CaO} \\
\mathrm{BaO} \\
\mathrm{Na} 20 \\
\mathrm{~K} 20\end{array}$ & $\begin{array}{c}63.0 \\
0.00 \\
9.2 \\
0.03 \\
0.08 \\
2.71 \\
0.00 \\
0.58 \\
2.75\end{array}$ & $\begin{array}{c}66.6 \\
0.00 \\
12.3 \\
0.01 \\
0.26 \\
3.37 \\
0.00 \\
0.55 \\
2.92\end{array}$ & $\begin{array}{c}67.4 \\
0.00 \\
12.2 \\
0.00 \\
0.25 \\
3.20 \\
0.00 \\
0.66 \\
3.10\end{array}$ & $\begin{array}{c}68.5 \\
0.00 \\
12.2 \\
0.05 \\
0.22 \\
3.21 \\
0.12 \\
0.63 \\
3.38\end{array}$ & $\begin{array}{c}68.0 \\
0.00 \\
12.2 \\
0.00 \\
0.23 \\
3.22 \\
0.00 \\
0.72 \\
2.93\end{array}$ & $\begin{array}{c}67.9 \\
0.00 \\
12.2 \\
0.00 \\
0.18 \\
3.24 \\
0.00 \\
0.68 \\
3.10\end{array}$ & $\begin{array}{c}62.6 \\
0.00 \\
11.2 \\
0.08 \\
0.61 \\
3.28 \\
0.00 \\
0.53 \\
1.82\end{array}$ & $\begin{array}{c}67.4 \\
0.00 \\
11.5 \\
0.00 \\
0.76 \\
3.59 \\
0.02 \\
0.43 \\
1.88\end{array}$ \\
\hline Total & 78.3 & 86.0 & 86.8 & 88.3 & 87.3 & 87.3 & 80.1 & 85.6 \\
\hline $\begin{array}{l}\mathrm{Si} \\
\mathrm{Ti} \\
\mathrm{Al} \\
\mathrm{Fe} \\
\mathrm{Mg} \\
\mathrm{Ca} \\
\mathrm{Ba} \\
\mathrm{Na} \\
\mathrm{K}\end{array}$ & $\begin{array}{c}30.7 \\
0.00 \\
5.29 \\
0.01 \\
0.06 \\
1.42 \\
0.00 \\
0.55 \\
1.71\end{array}$ & $\begin{array}{c}\text { Uni } \\
29.7 \\
0.00 \\
6.47 \\
0.00 \\
0.17 \\
1.61 \\
0.00 \\
0.48 \\
1.66\end{array}$ & $\begin{array}{c}\text { Ce } 11 \mathrm{Co} \\
29.8 \\
0.00 \\
6.36 \\
0.00 \\
0.16 \\
1.52 \\
0.00 \\
0.57 \\
1.75\end{array}$ & $\begin{array}{c}\text { ositior } \\
29.9 \\
0.00 \\
6.27 \\
0.02 \\
0.14 \\
1.50 \\
0.02 \\
0.53 \\
1.88\end{array}$ & $\begin{array}{c}\text { Based on } \\
29.9 \\
0.00 \\
6.32 \\
0.00 \\
0.15 \\
1.52 \\
0.00 \\
0.61 \\
1.64\end{array}$ & $\begin{array}{c}72(0) \\
29.9 \\
0.00 \\
6.32 \\
0.00 \\
0.12 \\
1.53 \\
0.00 \\
0.58 \\
1.74\end{array}$ & $\begin{array}{c}29.8 \\
0.00 \\
6.29 \\
0.03 \\
0.43 \\
1.67 \\
0.00 \\
0.49 \\
1.11\end{array}$ & $\begin{array}{c}30.0 \\
0.00 \\
6.04 \\
0.00 \\
0.50 \\
1.71 \\
0.00 \\
0.37 \\
1.07\end{array}$ \\
\hline \multicolumn{9}{|c|}{$\mathrm{Si} /(\mathrm{Al}+\mathrm{Fe})$} \\
\hline & \multicolumn{8}{|c|}{$(\mathrm{A} T+\mathrm{Fe}) /(2 \mathrm{Mg}+2 \mathrm{Ca}+2 \mathrm{Ba}+\mathrm{Na}+\mathrm{K})$} \\
\hline $\begin{array}{l}\mathrm{K} \\
\mathrm{Na} \\
\mathrm{Ca}+\mathrm{Mg}\end{array}$ & $\begin{array}{l}45.8 \\
14.7 \\
39.5\end{array}$ & $\begin{array}{l}42.4 \\
12.1 \\
45.5\end{array}$ & $\begin{array}{c}\text { Mol\% Ex } \\
43.8 \\
14.2 \\
42.1\end{array}$ & $\begin{array}{c}\text { nangeab } \\
46.4 \\
13.1 \\
40.5\end{array}$ & $\begin{array}{c}\text { Cations } \\
41.9 \\
15.6 \\
42.5\end{array}$ & $\begin{array}{l}43.9 \\
14.6 \\
41.5\end{array}$ & $\begin{array}{l}29.9 \\
13.2 \\
56.9\end{array}$ & $\begin{array}{l}29.2 \\
10.2 \\
60.6\end{array}$ \\
\hline
\end{tabular}




\section{APPENDIX $F$ (cont)}

Drill Hole USW H-4

\begin{tabular}{|c|c|c|c|c|c|c|c|c|}
\hline $\begin{array}{l}\text { Depth } \\
\text { ( ft) } \\
\text { Unit }\end{array}$ & $\begin{array}{r}1455 \\
\text { Tht }\end{array}$ & 1455 & $\begin{array}{r}1550 \\
\text { Tht }\end{array}$ & 1550 & 1550 & 1550 & 1550 & 1550 \\
\hline $\begin{array}{l}\mathrm{SiO} 02 \\
\mathrm{TiO} 02 \\
\mathrm{Al} 203 \\
\mathrm{Fe} 203 \\
\mathrm{MgO} \\
\mathrm{CaO} \\
\mathrm{BaO} \\
\mathrm{Na} 20 \\
\mathrm{~K} 20\end{array}$ & $\begin{array}{c}64.5 \\
0.00 \\
11.9 \\
0.00 \\
0.76 \\
3.53 \\
0.00 \\
0.42 \\
1.88\end{array}$ & $\begin{array}{c}66.4 \\
0.00 \\
12.2 \\
0.00 \\
0.77 \\
3.73 \\
0.03 \\
0.38 \\
1.40\end{array}$ & $\begin{array}{c}65.5 \\
0.00 \\
12.2 \\
0.00 \\
0.00 \\
2.20 \\
0.00 \\
1.54 \\
5.08\end{array}$ & $\begin{array}{c}63.9 \\
0.00 \\
11.9 \\
0.04 \\
0.00 \\
2.15 \\
0.09 \\
1.46 \\
4.61\end{array}$ & $\begin{array}{c}57.9 \\
0.00 \\
12.2 \\
0.03 \\
0.00 \\
2.26 \\
0.00 \\
1.79 \\
3.31\end{array}$ & $\begin{array}{c}64.9 \\
0.00 \\
11.7 \\
0.16 \\
0.00 \\
2.18 \\
0.00 \\
1.45 \\
4.52\end{array}$ & $\begin{array}{c}67.2 \\
0.00 \\
12.9 \\
0.02 \\
0.00 \\
2.44 \\
0.15 \\
1.30 \\
4.42\end{array}$ & $\begin{array}{c}68.8 \\
0.00 \\
12.9 \\
0.00 \\
0.00 \\
2.25 \\
0.15 \\
1.32 \\
4.54\end{array}$ \\
\hline Total & 83.0 & 84.9 & 87.5 & 84.2 & 77.5 & 84.9 & 88.4 & 90.0 \\
\hline $\begin{array}{l}\mathrm{Si} \\
\mathrm{Ti} \\
\mathrm{Al} \\
\mathrm{Fe} \\
\mathrm{Mg} \\
\mathrm{Ca} \\
\mathrm{Ba} \\
\mathrm{Na} \\
\mathrm{K}\end{array}$ & $\begin{array}{c}29.7 \\
0.00 \\
6.45 \\
0.00 \\
0.52 \\
1.74 \\
0.00 \\
0.37 \\
1.10\end{array}$ & $\begin{array}{c}\text { Uni } \\
29.7 \\
0.00 \\
6.44 \\
0.00 \\
0.51 \\
1.79 \\
0.01 \\
0.33 \\
0.80\end{array}$ & $\begin{array}{c}\text { Ce11 Co } \\
29.6 \\
0.00 \\
6.41 \\
0.00 \\
0.00 \\
1.05 \\
0.00 \\
1.33 \\
2.89\end{array}$ & $\begin{array}{c}\text { osition } \\
29.6 \\
0.00 \\
6.49 \\
0.01 \\
0.00 \\
1.07 \\
0.02 \\
1.31 \\
2.72\end{array}$ & $\begin{array}{c}\text { Based on } \\
29.0 \\
0.00 \\
7.21 \\
0.01 \\
0.00 \\
1.21 \\
0.00 \\
1.74 \\
2.12\end{array}$ & $\begin{array}{c}72(0) \\
29.7 \\
0.00 \\
6.31 \\
0.06 \\
0.00 \\
1.07 \\
0.00 \\
1.29 \\
2.64\end{array}$ & $\begin{array}{c}29.5 \\
0.00 \\
6.68 \\
0.01 \\
0.00 \\
1.15 \\
0.03 \\
1.11 \\
2.48\end{array}$ & $\begin{array}{c}29.7 \\
0.00 \\
6.55 \\
0.00 \\
0.00 \\
1.04 \\
0.03 \\
1.10 \\
2.50\end{array}$ \\
\hline \multicolumn{9}{|c|}{$\mathrm{Si} /(\mathrm{Al}+\mathrm{Fe})$} \\
\hline \multicolumn{9}{|c|}{$(\mathrm{Al}+\mathrm{Fe}) /(2 \mathrm{Mg}+2 \mathrm{Ca}+2 \mathrm{Ba}+\mathrm{Na}+\mathrm{K})$} \\
\hline & 1.08 & 1.12 & 1.01 & 1.05 & 1.15 & 1.05 & 1.13 & 1.14 \\
\hline $\begin{array}{l}\mathrm{K} \\
\mathrm{Na} \\
\mathrm{Ca}+\mathrm{Mg}\end{array}$ & $\begin{array}{l}29.5 \\
10.0 \\
60.5\end{array}$ & $\begin{array}{r}23.3 \\
9.6 \\
67.1\end{array}$ & $\begin{array}{l}\text { Mo1\% Ex } \\
54.8 \\
25.3 \\
19.9\end{array}$ & $\begin{array}{c}\text { angeab1 } \\
53.4 \\
25.7 \\
20.9\end{array}$ & $\begin{array}{c}\text { Cations } \\
41.7 \\
34.3 \\
23.9\end{array}$ & $\begin{array}{l}52.8 \\
25.8 \\
21.4\end{array}$ & $\begin{array}{l}52.3 \\
23.4 \\
24.3\end{array}$ & $\begin{array}{l}53.8 \\
23.8 \\
22.4\end{array}$ \\
\hline
\end{tabular}




\section{APPENDIX F (cont) \\ Drill Hole USW H-4}

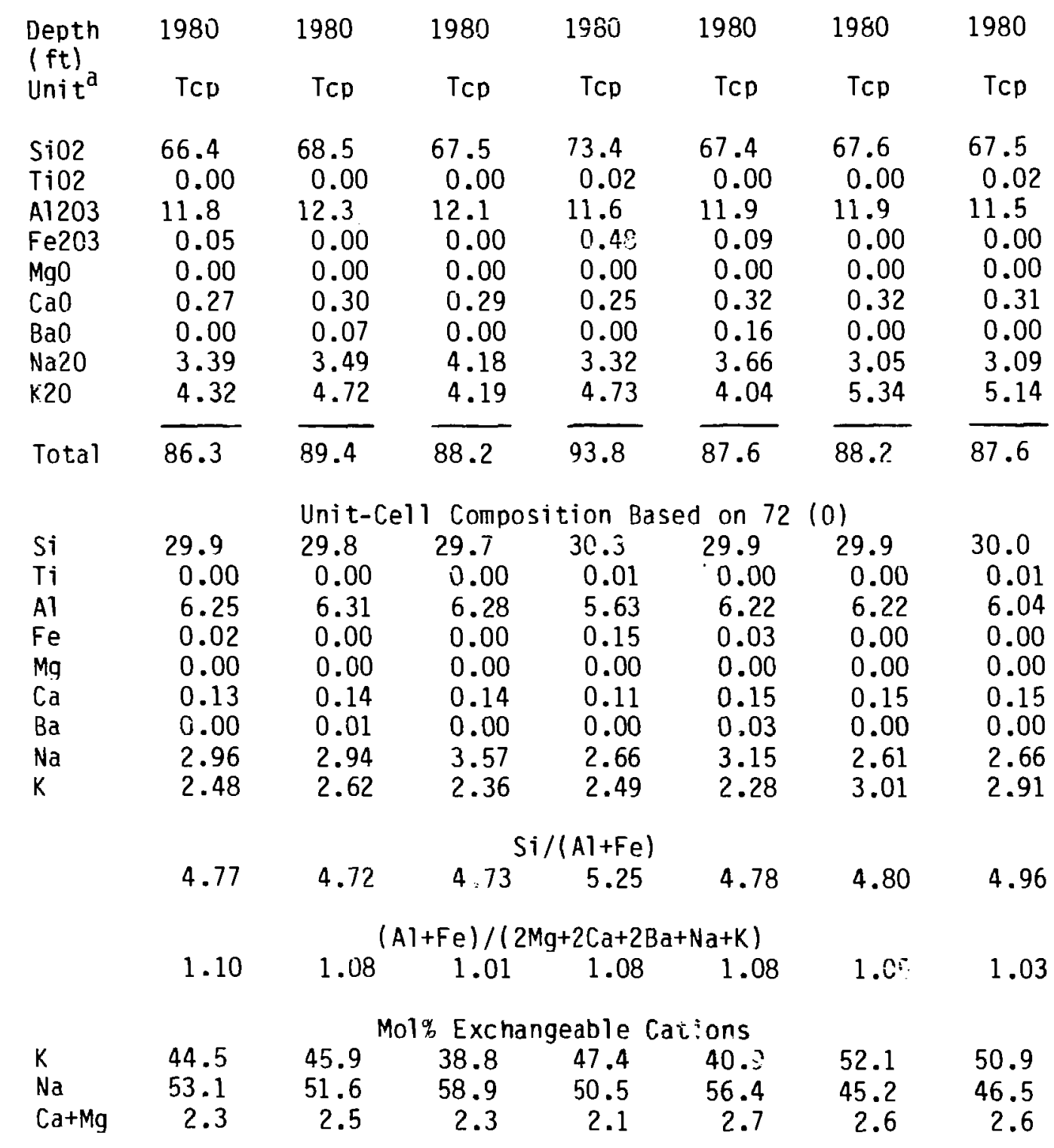


Drill Hole USW H-5

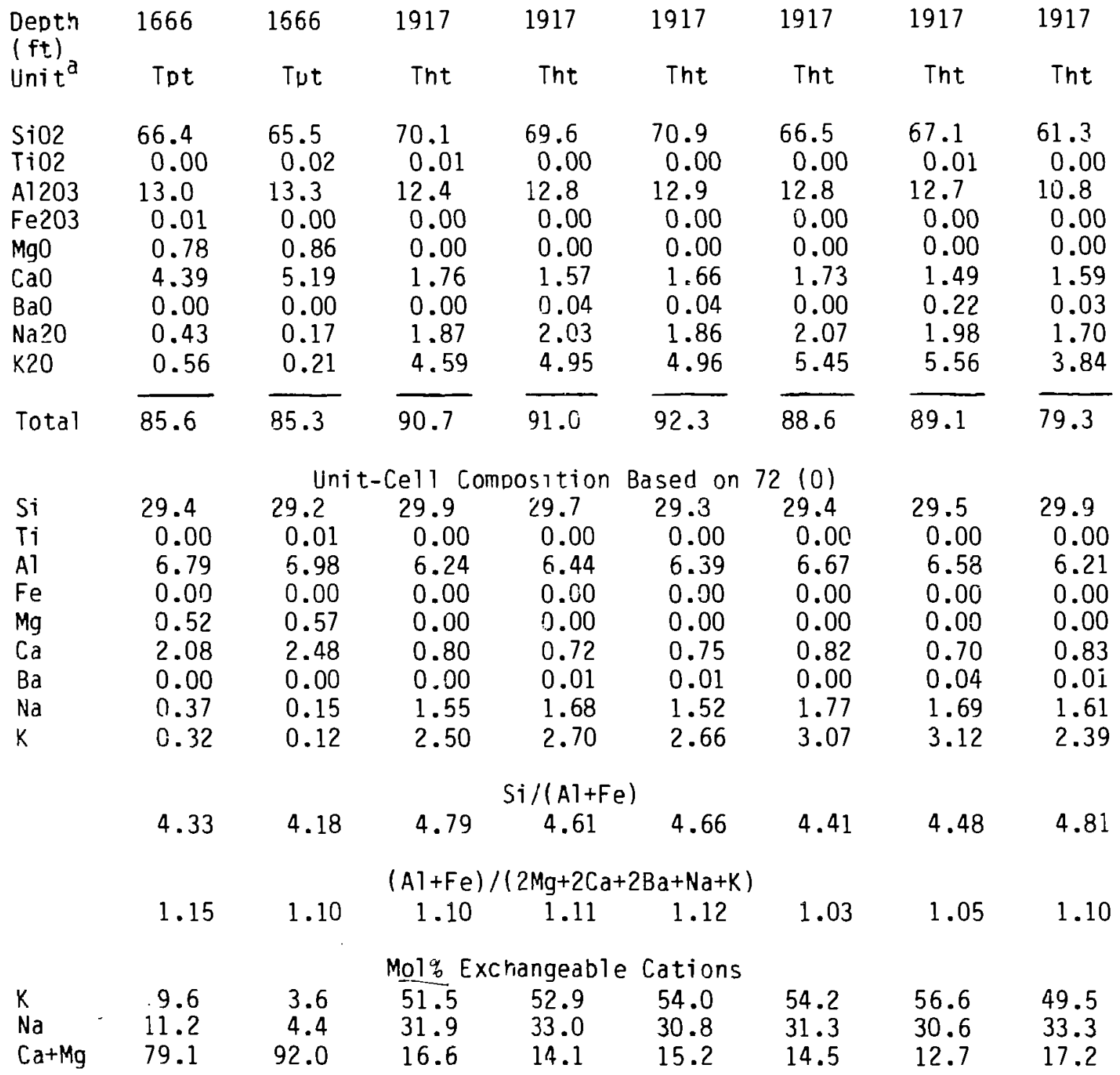


APPENDIX $F$ (cont)

Drill Hole USW H-5

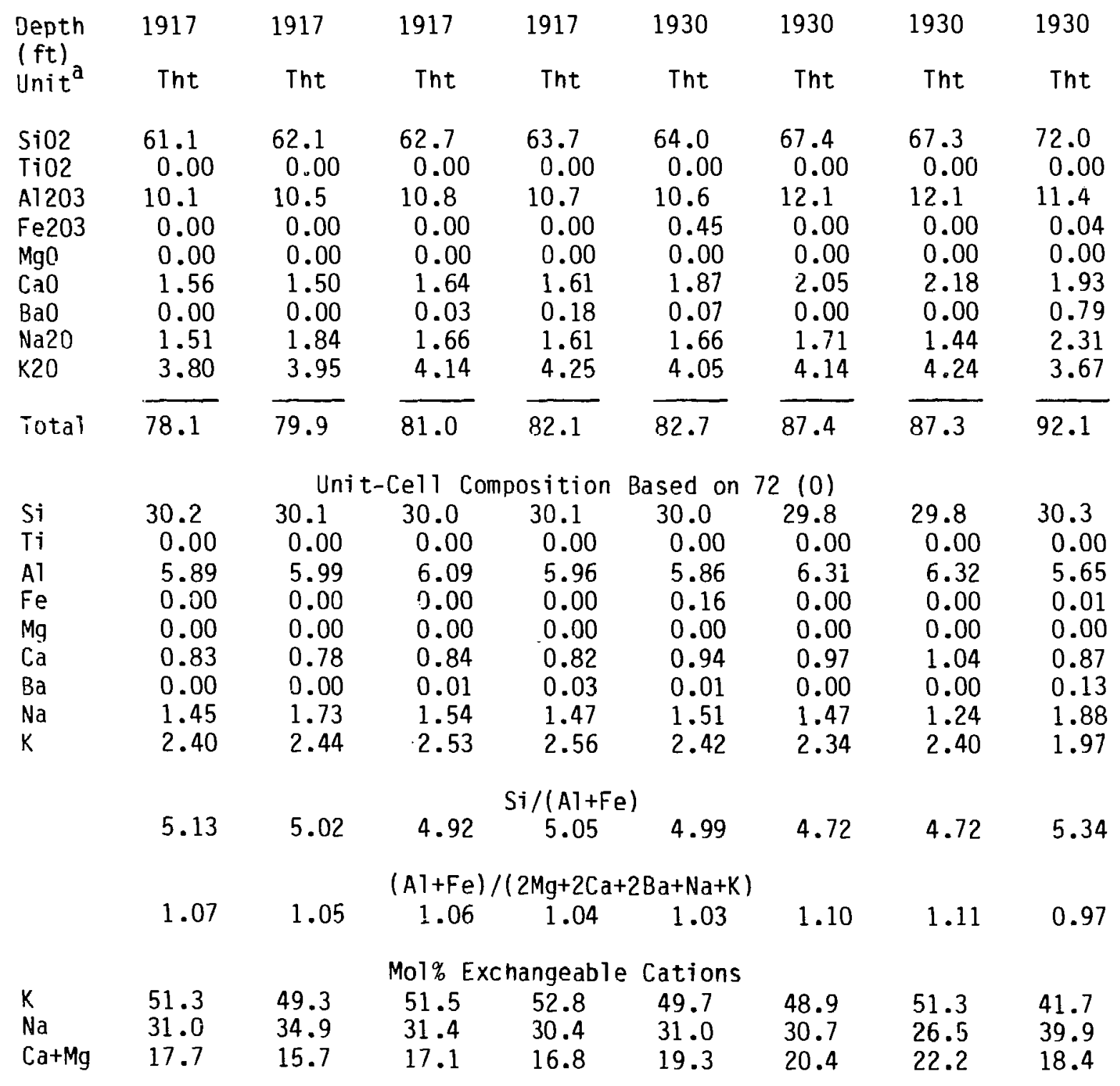


APPENDIX $F$ (cont)

Orill Hole USW H-5

$\begin{array}{lcccc}\begin{array}{l}\text { Depth } \\ \text { (ft) }\end{array} & 2200 & 2200 & 2200 & 2200 \\ \text { Unit } & \text { Tcp } & \text { Tco } & \text { Tcp } & \text { Tcp } \\ \text { Si02 } & 71.3 & 62.2 & 68.8 & 71.4 \\ \text { Ti02 } & 0.00 & 0.00 & 0.00 & 0.00 \\ \text { A1203 } & 11.0 & 12.4 & 11.0 & 12.4 \\ \text { Fe203 } & 0.00 & 0.01 & 0.00 & 0.00 \\ \text { Mg0 } & 0.04 & 0.00 & 0.00 & 0.00 \\ \text { Ca0 } & 1.31 & 1.34 & 1.55 & 1.62 \\ \text { Ba0 } & 0.01 & 0.00 & 0.00 & 0.23 \\ \text { Na20 } & 2.49 & 2.80 & 2.22 & 2.20 \\ \text { K20 } & 3.24 & 3.89 & 3.63 & 4.53 \\ \text { Total } & \frac{39.4}{82.6} & \frac{87.2}{92.4} & \frac{92.4}{}\end{array}$

Unit-Cell Composition Based on 72 (0)

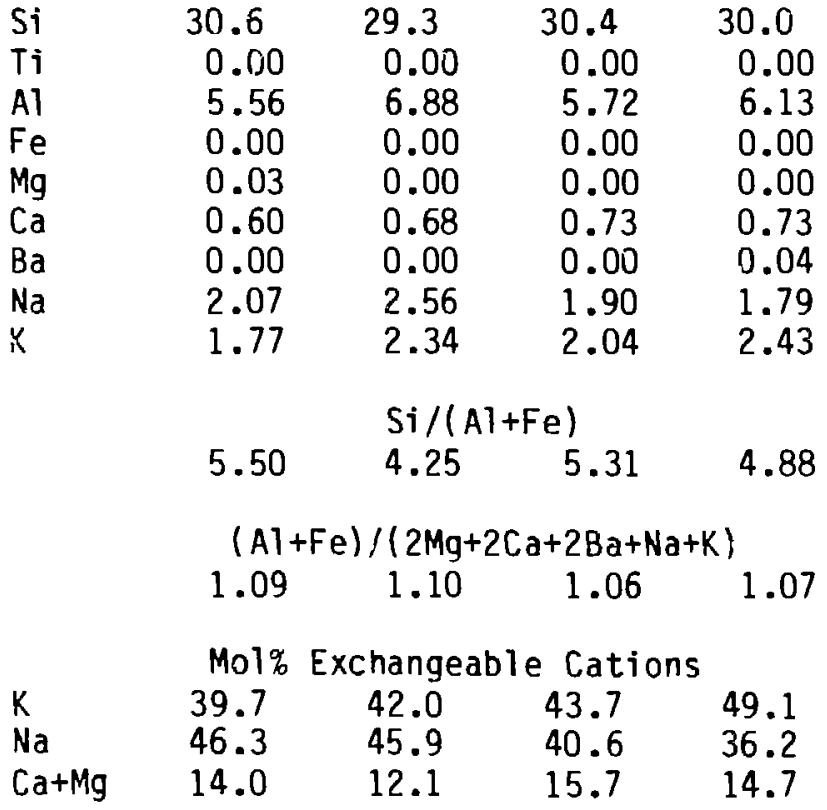




\section{APPENDIK $F$ (cont)}

Drill Hole UE-25a\#1

\begin{tabular}{|c|c|c|c|c|c|c|c|c|}
\hline $\begin{array}{l}\text { Depth } \\
\text { (ft) } \\
\text { Unit }\end{array}$ & 1279 & 1323 & 1323 & 1323 & 1323 & 1323 & 1323 & 1323 \\
\hline $\begin{array}{l}\mathrm{SiO} 02 \\
\mathrm{TiO} 02 \\
\mathrm{~A} 1203 \\
\mathrm{Fe} 203 \\
\mathrm{MgO} \\
\mathrm{CaO} \\
\mathrm{BaO} \\
\mathrm{Na} 20 \\
\mathrm{~K} 20\end{array}$ & $\begin{array}{c}55.7 \\
0.00 \\
14.1 \\
0.82 \\
3.30 \\
2.10 \\
0.00 \\
0.62 \\
0.85\end{array}$ & $\begin{array}{c}65.8 \\
0.00 \\
11.4 \\
0.00 \\
0.74 \\
3.87 \\
0.00 \\
0.50 \\
1.75\end{array}$ & $\begin{array}{c}68.9 \\
0.00 \\
12.0 \\
0.02 \\
0.62 \\
3.80 \\
0.13 \\
0.55 \\
2.02\end{array}$ & $\begin{array}{c}69.0 \\
0.00 \\
12.3 \\
0.08 \\
0.60 \\
3.85 \\
0.00 \\
0.52 \\
1.81\end{array}$ & $\begin{array}{c}68 . \bar{j} \\
0.00 \\
12.0 \\
0.02 \\
0.57 \\
3.92 \\
0.00 \\
0.56 \\
1.76\end{array}$ & $\begin{array}{c}68.7 \\
0.02 \\
12.3 \\
0.04 \\
0.53 \\
4.20 \\
0.06 \\
0.56 \\
1.43\end{array}$ & $\begin{array}{c}66.1 \\
0.03 \\
11.6 \\
0.02 \\
0.38 \\
4.01 \\
0.15 \\
0.67 \\
1.78\end{array}$ & $\begin{array}{c}66.4 \\
0.03 \\
12.0 \\
0.02 \\
0.67 \\
3.92 \\
0.20 \\
0.60 \\
1.80\end{array}$ \\
\hline Total & 77.4 & 84.1 & 88.1 & 88.1 & 87.6 & 87.8 & 84.7 & 85.6 \\
\hline $\begin{array}{l}\mathrm{Si} \\
\mathrm{Ti} \\
\mathrm{Al} \\
\mathrm{Fe} \\
\mathrm{Mg} \\
\mathrm{Ca} \\
\mathrm{Ba} \\
\mathrm{Na} \\
\mathrm{K}\end{array}$ & $\begin{array}{c}27.6 \\
0.00 \\
8.22 \\
0.31 \\
2.43 \\
1.11 \\
0.00 \\
0.59 \\
0.54\end{array}$ & $\begin{array}{l}\text { Uni } \\
29.9 \\
0.00 \\
6.09 \\
0.00 \\
0.50 \\
1.88 \\
0.00 \\
0.44 \\
1.01\end{array}$ & $\begin{array}{c}\text { Cel1 Cc } \\
29.9 \\
0.00 \\
6.14 \\
0.01 \\
0.40 \\
1.77 \\
0.02 \\
0.40 \\
1.12\end{array}$ & $\begin{array}{c}\text { ositior } \\
29.8 \\
0.00 \\
6.25 \\
0.03 \\
0.39 \\
1.79 \\
0.00 \\
0.44 \\
1.00\end{array}$ & $\begin{array}{c}\text { Based on } \\
29.9 \\
0.00 \\
6.17 \\
0.01 \\
0.37 \\
1.83 \\
0.00 \\
0.47 \\
0.98\end{array}$ & $\begin{array}{c}72(0) \\
29.8 \\
0.01 \\
6.28 \\
0.01 \\
0.34 \\
1.95 \\
0.01 \\
0.47 \\
0.79\end{array}$ & $\begin{array}{c}29.8 \\
0.01 \\
6.20 \\
0.01 \\
0.26 \\
1.94 \\
0.03 \\
0.59 \\
1.03\end{array}$ & $\begin{array}{c}29.7 \\
0.01 \\
6.30 \\
0.01 \\
0.45 \\
1.88 \\
0.04 \\
0.52 \\
1.03\end{array}$ \\
\hline & 3.23 & 4.91 & 4.87 & $\begin{array}{r}i /(A)+1 \\
4.76\end{array}$ & 4.84 & 4.74 & 4.81 & 4.71 \\
\hline \multicolumn{2}{|c|}{$(\mathrm{Al}+\mathrm{Fe}) /(2 \mathrm{Mg}+2 \mathrm{Ca}+2 \mathrm{Ba}+\mathrm{Na}+\mathrm{K})$} & & $\begin{array}{r}(A]+F e) \\
1.03\end{array}$ & $\begin{array}{r}\mathrm{Mg}+2 \mathrm{Ca} \\
1.09\end{array}$ & $\begin{array}{c}(\mathrm{Ba}+\mathrm{Na}+\mathrm{K}) \\
1.06\end{array}$ & 1.07 & 1.02 & 1.01 \\
\hline $\begin{array}{l}\mathrm{K} \\
\mathrm{Na} \\
\mathrm{Ca}+\mathrm{Mg}\end{array}$ & $\begin{array}{l}11.5 \\
12.7 \\
75.8\end{array}$ & $\begin{array}{l}26.4 \\
11.5 \\
62.1\end{array}$ & $\begin{array}{c}\text { Mol\% E } \\
29.8 \\
12.3 \\
57.8\end{array}$ & $\begin{array}{c}\text { langeab } \\
27.7 \\
12.1 \\
60.2\end{array}$ & $\begin{array}{c}\text { Cations } \\
26.8 \\
13.0 \\
60.3\end{array}$ & $\begin{array}{l}22.2 \\
13.2 \\
64.5\end{array}$ & $\begin{array}{l}26.9 \\
15.4 \\
57.7\end{array}$ & $\begin{array}{l}26.5 \\
13.4 \\
60.0\end{array}$ \\
\hline
\end{tabular}




\section{APPENDIX $F$ (cont)}

\section{Drill Hole UE-25a\#1}

\begin{tabular}{|c|c|c|c|c|c|c|c|c|}
\hline $\begin{array}{l}\text { Depth } \\
\text { (ft) } \\
\text { Unit }\end{array}$ & 1358 & 1358 & 1358 & 1381 & 1384 & 1384 & 1384 & 1384 \\
\hline $\begin{array}{l}\mathrm{SiO} 2 \\
\mathrm{TiO} 2 \\
\mathrm{Al} 203 \\
\mathrm{Fe} 203 \\
\mathrm{MgO} \\
\mathrm{CaO} \\
\mathrm{BaO} \\
\mathrm{Na} 20 \\
\mathrm{~K} 20\end{array}$ & $\begin{array}{c}67.7 \\
0.00 \\
11.2 \\
0.03 \\
0.31 \\
3.71 \\
0.07 \\
0.57 \\
1.79\end{array}$ & $\begin{array}{c}63.8 \\
0.00 \\
10.3 \\
0.23 \\
0.22 \\
3.07 \\
0.03 \\
0.56 \\
2.05\end{array}$ & $\begin{array}{c}66.7 \\
0.00 \\
11.2 \\
0.00 \\
0.30 \\
3.80 \\
0.10 \\
0.35 \\
1.78\end{array}$ & $\begin{array}{c}67.0 \\
0.03 \\
11.9 \\
0.00 \\
0.69 \\
3.74 \\
0.09 \\
0.48 \\
1.83\end{array}$ & $\begin{array}{c}65.9 \\
0.02 \\
11.8 \\
0.00 \\
0.68 \\
3.50 \\
0.12 \\
0.60 \\
2.23\end{array}$ & $\begin{array}{c}66.5 \\
0.02 \\
11.6 \\
0.00 \\
0.75 \\
3.73 \\
0.00 \\
0.51 \\
1.86\end{array}$ & $\begin{array}{c}65.4 \\
0.00 \\
11.7 \\
0.00 \\
0.68 \\
4.09 \\
0.08 \\
0.49 \\
1.50\end{array}$ & $\begin{array}{c}67.6 \\
0.04 \\
10.9 \\
0.00 \\
0.62 \\
3.96 \\
0.00 \\
0.50 \\
1.65\end{array}$ \\
\hline Total & 85.4 & 80.2 & 84.2 & 85.7 & 84.8 & 85.0 & 84.0 & 85.2 \\
\hline $\begin{array}{l}\mathrm{Si} \\
\mathrm{Ti} \\
\mathrm{Al} \\
\mathrm{Fe} \\
\mathrm{Mg} \\
\mathrm{Ca} \\
\mathrm{Ba} \\
\mathrm{Na} \\
\mathrm{K}\end{array}$ & $\begin{array}{c}30.2 \\
0.00 \\
5.91 \\
0.01 \\
0.21 \\
1.77 \\
0.01 \\
0.49 \\
1.02\end{array}$ & $\begin{array}{c}\text { Uni } \\
30.3 \\
0.00 \\
5.74 \\
0.08 \\
0.16 \\
1.56 \\
0.01 \\
0.52 \\
1.24\end{array}$ & $\begin{array}{c}\text { Cel 1 C } \\
30.2 \\
0.00 \\
5.98 \\
0.00 \\
0.20 \\
1.84 \\
0.02 \\
0.31 \\
1.03\end{array}$ & $\begin{array}{c}\text { ositiol } \\
29.8 \\
0.01 \\
5.22 \\
0.00 \\
0.46 \\
1.78 \\
0.02 \\
0.41 \\
1.04\end{array}$ & $\begin{array}{c}\text { Based } 0 \\
29.7 \\
0.01 \\
6.27 \\
0.00 \\
0.46 \\
1.69 \\
0.02 \\
0.53 \\
1.28\end{array}$ & $\begin{array}{c}72(0) \\
29.9 \\
0.01 \\
6.13 \\
0.00 \\
0.50 \\
1.80 \\
0.00 \\
0.44 \\
1.07\end{array}$ & $\begin{array}{c}29.7 \\
0.00 \\
6.25 \\
0.00 \\
0.46 \\
1.99 \\
0.01 \\
0.43 \\
0.92\end{array}$ & $\begin{array}{c}30.2 \\
0.01 \\
5.73 \\
0.00 \\
0.41 \\
1.90 \\
0.00 \\
0.43 \\
0.94\end{array}$ \\
\hline \multicolumn{9}{|c|}{$\mathrm{Si} /(\mathrm{A})+\mathrm{Fe})$} \\
\hline \multicolumn{9}{|c|}{$(\mathrm{A} 1+\mathrm{Fe}) /(2 \mathrm{Mg}+2 \mathrm{Ca}+2 \mathrm{Ba}+\mathrm{Na}+\mathrm{K})$} \\
\hline $\begin{array}{l}\mathrm{K} \\
\mathrm{Na} \\
\mathrm{Ca}+\mathrm{Mg}\end{array}$ & $\begin{array}{l}29.2 \\
14.1 \\
56.7\end{array}$ & $\begin{array}{l}35.7 \\
14.8 \\
49.4\end{array}$ & $\begin{array}{c}\text { Mo1\% E } \\
30.4 \\
9.1 \\
60.5\end{array}$ & $\begin{array}{c}\text { angeab } \\
28.1 \\
11.2 \\
60.7\end{array}$ & $\begin{array}{c}\text { Cation } \\
32.4 \\
13.3 \\
54.3\end{array}$ & $\begin{array}{l}28.0 \\
11.7 \\
60.3\end{array}$ & $\begin{array}{l}24.1 \\
11.4 \\
64.5\end{array}$ & $\begin{array}{l}25.5 \\
11.8 \\
62.7\end{array}$ \\
\hline
\end{tabular}




\section{APPENDIX $F$ (cont)}

Drill Hole UE-25a\#1

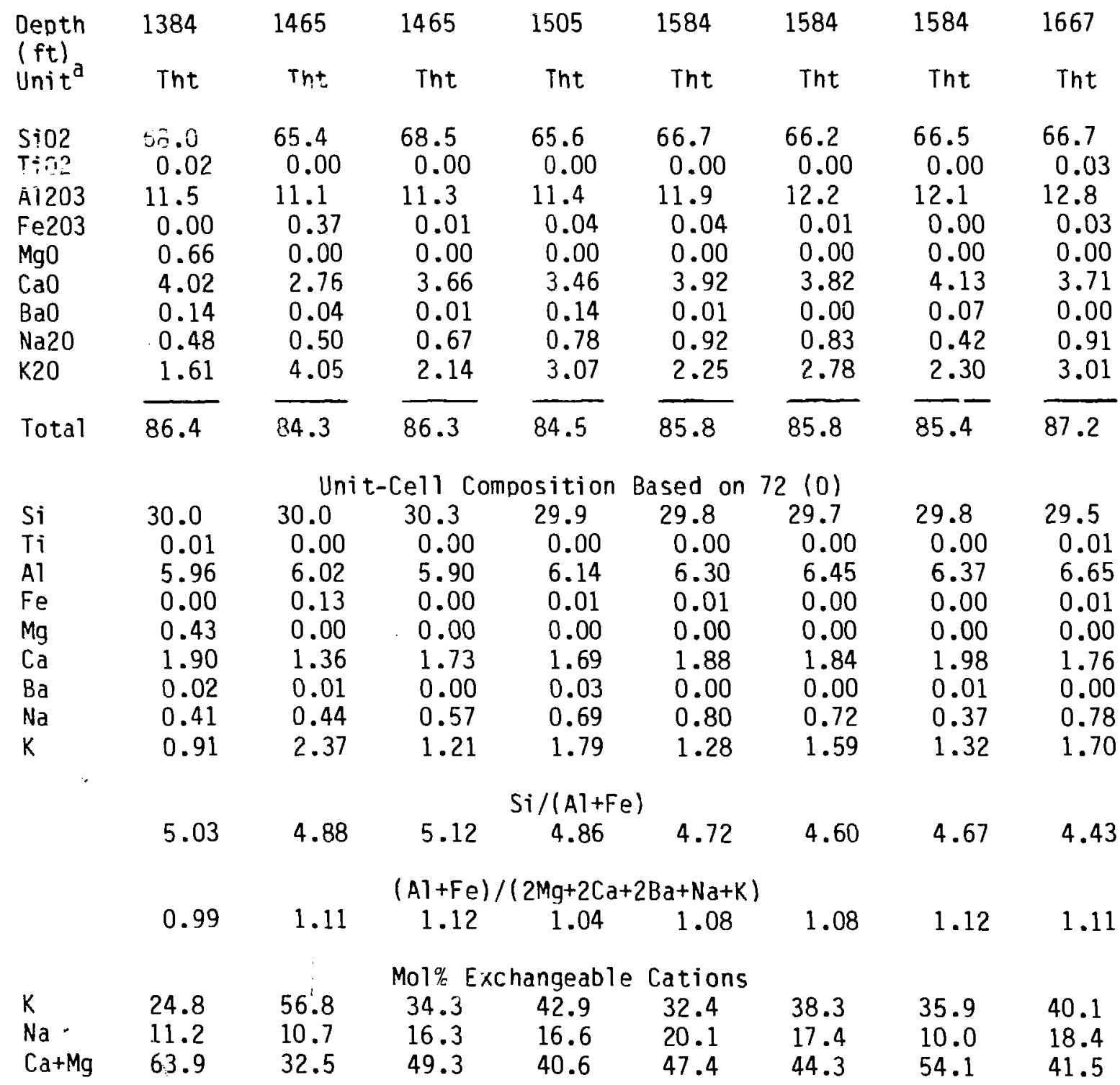




\section{APPENDIX F (cont)}

\section{Drill Hole UE-25a\#1}

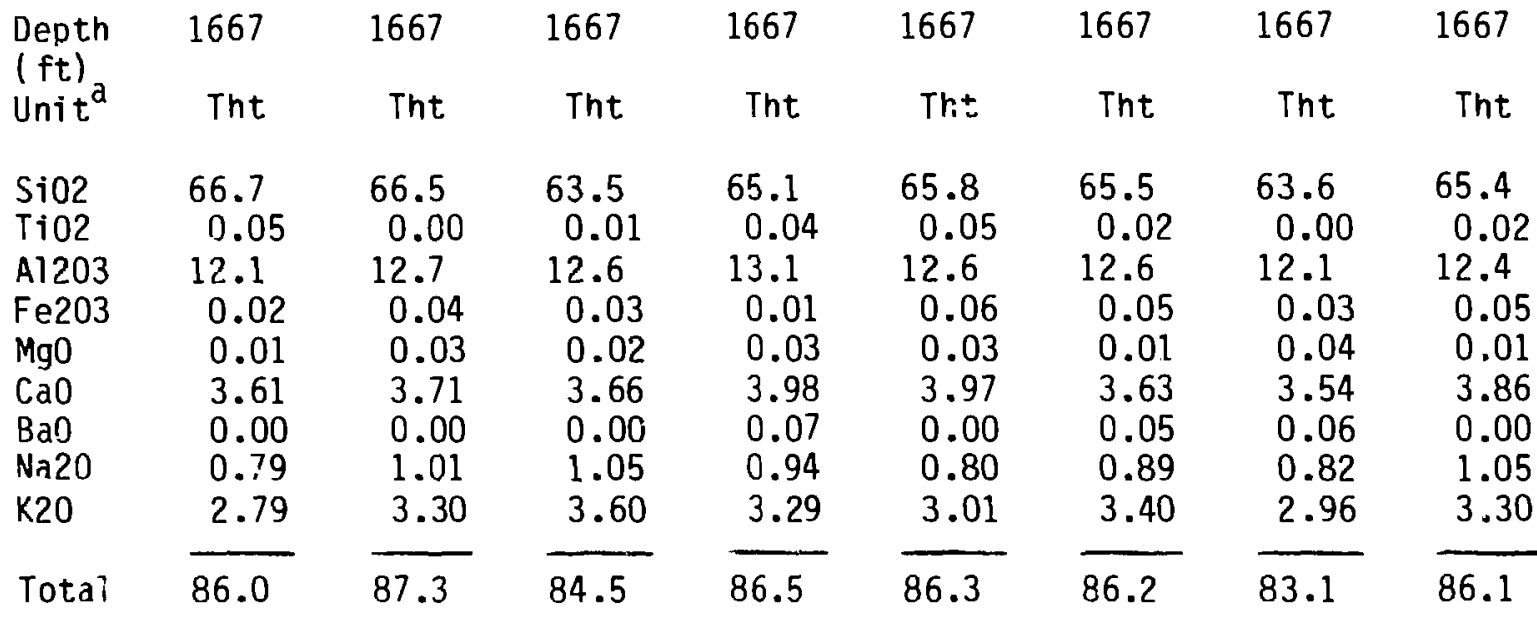

Si

$\mathrm{Ti}$

A1

$\mathrm{Fe}$

$\mathrm{Mg}$

Ca

$\mathrm{Ba}$

$\mathrm{Na}$

$\mathrm{K}$

$k$

$\mathrm{Na}$

$\mathrm{Ca}+\mathrm{Mg}$
Unit-Cell Composition Based on 72 (0) $\begin{array}{lllll}29.5 & 29.2 & 29.2 & 29.4 & 29.4\end{array}$

$$
29.8
$$$$
0.02
$$$$
0.00
$$$$
6.36
$$

6.62

0.00

0.01

0.02

6.65

6.90

0.02

0.01

0.00

0.02

0.02

1.91

1.80

0.01

1.90

0.82

0.94

1.88

0.00

0.69

1.72

0.01

6.68

0.02

0.01

1.75

0.01

0.77

1.95

29.5

0.00

6.59

0.01

0.03

1.76

0.01

0.74

1.75

29.4

0.01

6.57

0.02

1.59

1.86

2.11

$\mathrm{Si} /(\mathrm{Al}+\mathrm{Fe})$

4.68

4.44

$$
4.26
$$

$4.2{ }^{\prime}$

4.41

4.40

4.47

4.47

$(\mathrm{Al}+\mathrm{Fe}) /(2 \mathrm{Mg}+2 \mathrm{Ca}+2 \mathrm{Ba}+\mathrm{Na}+\mathrm{K})$

1.11

1.05

1.03

1.05

1.07

1.07

1.08

1.01

Mol\% Exchangeable Cations

$\begin{array}{lllllllll} & 39.7 & 41.3 & 43.4 & 40.6 & 39.6 & 43.5 & 41.0 & 40.5 \\ \mathrm{Na} & 17.1 & 19.2 & 19.2 & 17.6 & 16.0 & 17.3 & 17.2 & 19.6 \\ \mathrm{Ca}+\mathrm{Mg} & 43.3 & 39.5 & 37.3 & 41.7 & 44.4 & 39.2 & 41.8 & 39.9\end{array}$


APPENDIX $F$ (cont)

Drill Hole UE-25a\#1

\begin{tabular}{|c|c|c|c|c|c|c|c|c|}
\hline $\begin{array}{l}\text { Depth } \\
\text { (ft) } \\
\text { Unit }\end{array}$ & 1667 & 1667 & 1667 & $\begin{array}{l}1824 \\
\text { Tht }\end{array}$ & 1824 & 1824 & 1824 & $\begin{array}{r}2087 \\
\text { TCD }\end{array}$ \\
\hline $\begin{array}{l}\mathrm{SiO} 2 \\
\mathrm{TiO} 02 \\
\mathrm{Al} 203 \\
\mathrm{Fe} 203 \\
\mathrm{MgO} \\
\mathrm{CaO} \\
\mathrm{BaO} \\
\mathrm{Na} 20 \\
\mathrm{~K} 2 \mathrm{O}\end{array}$ & $\begin{array}{c}66.6 \\
0.05 \\
12.6 \\
0.06 \\
0.03 \\
3.97 \\
0.00 \\
0.80 \\
3.01\end{array}$ & $\begin{array}{c}69.2 \\
0.00 \\
13.3 \\
0.08 \\
0.04 \\
4.03 \\
0.04 \\
0.89 \\
2.82\end{array}$ & $\begin{array}{c}67.1 \\
0.00 \\
12.6 \\
0.06 \\
0.02 \\
3.84 \\
0.32 \\
1.02 \\
2.92\end{array}$ & $\begin{array}{c}62.5 \\
0.00 \\
12.6 \\
0.05 \\
0.08 \\
4.78 \\
0.00 \\
0.62 \\
1.00\end{array}$ & $\begin{array}{c}66.1 \\
0.00 \\
13.9 \\
0.03 \\
0.15 \\
5.43 \\
0.21 \\
1.13 \\
1.33\end{array}$ & $\begin{array}{c}68.8 \\
0.00 \\
13.4 \\
0.02 \\
0.14 \\
5.12 \\
0.19 \\
0.99 \\
1.44\end{array}$ & $\begin{array}{c}66.8 \\
0.02 \\
13.6 \\
0.00 \\
0.15 \\
4.96 \\
0.02 \\
1.22 \\
1.29\end{array}$ & $\begin{array}{c}65.3 \\
0.00 \\
11.6 \\
0.00 \\
0.52 \\
3.93 \\
0.00 \\
0.26 \\
1.40\end{array}$ \\
\hline Tot.al & 87.2 & 90.4 & 87.8 & 81.6 & 88.3 & 90.2 & 88.1 & 83.0 \\
\hline $\begin{array}{l}\mathrm{Si} \\
\mathrm{Ti} \\
\mathrm{Al} \\
\mathrm{Fe} \\
\mathrm{Mg} \\
\mathrm{Ca} \\
\mathrm{Ba} \\
\mathrm{Na} \\
\mathrm{K}\end{array}$ & $\begin{array}{c}29.5 \\
0.02 \\
6.59 \\
0.02 \\
0.02 \\
1.88 \\
0.00 \\
0.69 \\
1.70\end{array}$ & $\begin{array}{c}\text { Un } \\
29.5 \\
0.00 \\
6.68 \\
0.03 \\
0.03 \\
1.84 \\
0.01 \\
0.73 \\
1.53\end{array}$ & $\begin{array}{c}\text { Ce11 C } \\
29.5 \\
0.00 \\
6.52 \\
0.02 \\
0.01 \\
1.81 \\
0.06 \\
0.87 \\
1.64\end{array}$ & $\begin{array}{c}\text { position } \\
29.3 \\
0.00 \\
6.94 \\
0.02 \\
0.06 \\
2.40 \\
0.00 \\
0.56 \\
0.60\end{array}$ & $\begin{array}{c}\text { Based on } \\
28.9 \\
0.00 \\
7.17 \\
0.01 \\
0.10 \\
2.54 \\
0.04 \\
0.96 \\
0.74\end{array}$ & $\begin{array}{cc}72(0) \\
29.3 \\
0.00 \\
6.73 \\
0.01 \\
0.09 \\
2.34 \\
0.03 \\
0.82 \\
0.78\end{array}$ & $\begin{array}{c}29.1 \\
0.01 \\
6.98 \\
0.00 \\
0.10 \\
2.31 \\
0.00 \\
1.03 \\
0.72\end{array}$ & $\begin{array}{c}29.9 \\
0.00 \\
6.28 \\
0.00 \\
0.35 \\
1.93 \\
0.00 \\
0.23 \\
0.82\end{array}$ \\
\hline \multicolumn{9}{|c|}{$\begin{array}{c}\mathrm{Si} /(\mathrm{Al}+\mathrm{Fe}) \\
4.20\end{array}$} \\
\hline \multicolumn{9}{|c|}{$(\mathrm{A} 1+\mathrm{Fe}) /(2 \mathrm{Mg}+2 \mathrm{Ca}+2 \mathrm{Ba}+\mathrm{Na}+\mathrm{K})$} \\
\hline $\begin{array}{l}\mathrm{K} \\
\mathrm{Na} \\
\mathrm{Ca}+\mathrm{Mg}\end{array}$ & $\begin{array}{l}39.6 \\
16.0 \\
44.4\end{array}$ & $\begin{array}{l}37.1 \\
17.8 \\
45.1\end{array}$ & $\begin{array}{c}\text { Mo1\% E } \\
37.8 \\
20.1 \\
42.1\end{array}$ & $\begin{array}{l}\text { hangeab1 } \\
16.5 \\
15.6 \\
67.9\end{array}$ & $\begin{array}{c}\text { Cations } \\
17.1 \\
22.1 \\
60.8\end{array}$ & $\begin{array}{l}19.4 \\
20.3 \\
60.3\end{array}$ & $\begin{array}{l}17.2 \\
24.8 \\
58.0\end{array}$ & $\begin{array}{r}24.5 \\
6.9 \\
68.5\end{array}$ \\
\hline
\end{tabular}


APPENDIX $F$ (cont)

Drill Hole UE-25a\#1

\begin{tabular}{|c|c|c|c|c|c|c|c|c|}
\hline $\begin{array}{l}\text { Depth } \\
(\mathrm{ft}) \\
\text { Unit }\end{array}$ & 2087 & 2087 & 2087 & 2087 & 2113 & 2220 & 2220 & 2220 \\
\hline $\begin{array}{l}\mathrm{SiO} 2 \\
\mathrm{TiO} 2 \\
\mathrm{Al} 20 \\
\mathrm{Fe} 203 \\
\mathrm{MgO} \\
\mathrm{CaO} \\
\mathrm{BaO} \\
\mathrm{Na} 20 \\
\mathrm{~K} 2 \mathrm{O}\end{array}$ & $\begin{array}{c}64.1 \\
0.00 \\
12.0 \\
0.00 \\
0.71 \\
3.78 \\
0.05 \\
0.47 \\
1.54\end{array}$ & $\begin{array}{c}60.8 \\
0.00 \\
12.0 \\
0.05 \\
0.71 \\
3.92 \\
0.18 \\
0.59 \\
1.49\end{array}$ & $\begin{array}{c}66.3 \\
0.00 \\
12.0 \\
0.05 \\
0.68 \\
3.94 \\
0.05 \\
0.36 \\
1.29\end{array}$ & $\begin{array}{c}63.5 \\
0.02 \\
12.4 \\
0.02 \\
0.60 \\
4.09 \\
0.19 \\
0.70 \\
2.14\end{array}$ & $\begin{array}{c}75.1 \\
0.00 \\
11.4 \\
0.29 \\
0.00 \\
0.87 \\
0.00 \\
3.11 \\
4.59\end{array}$ & $\begin{array}{c}64.8 \\
0.00 \\
11.5 \\
0.00 \\
0.60 \\
3.70 \\
0.00 \\
0.41 \\
1.48\end{array}$ & $\begin{array}{c}65.4 \\
0.00 \\
11.7 \\
0.00 \\
0.61 \\
3.76 \\
0.00 \\
0.33 \\
1.33\end{array}$ & $\begin{array}{c}65.0 \\
0.00 \\
11.6 \\
0.00 \\
0.54 \\
3.87 \\
0.00 \\
0.35 \\
1.25\end{array}$ \\
\hline Total & 82.7 & 79.7 & 84.7 & 83.6 & 95.4 & 82.5 & 83.2 & 82.6 \\
\hline $\begin{array}{l}\mathrm{Si} \\
\mathrm{Ti} \\
\mathrm{Al} \\
\mathrm{Fe} \\
\mathrm{Mg} \\
\mathrm{Ca} \\
\mathrm{Ba} \\
\mathrm{Na} \\
\mathrm{K}\end{array}$ & $\begin{array}{c}29.6 \\
0.00 \\
5.52 \\
0.00 \\
0.49 \\
1.87 \\
0.01 \\
0.42 \\
0.91\end{array}$ & $\begin{array}{c}\text { Uni } \\
29.2 \\
0.00 \\
6.80 \\
0.02 \\
0.51 \\
2.02 \\
0.03 \\
0.55 \\
0.91\end{array}$ & $\begin{array}{c}\text { Ce11 Cc } \\
29.8 \\
0.00 \\
6.37 \\
0.02 \\
0.46 \\
1.90 \\
0.01 \\
0.31 \\
0.74\end{array}$ & $\begin{array}{c}\text { osition } \\
29.2 \\
0.01 \\
6.72 \\
0.01 \\
0.41 \\
2.02 \\
0.03 \\
0.63 \\
1.26\end{array}$ & $\begin{array}{c}\text { Based on } \\
3.4 \\
0.00 \\
0.61 \\
0.01 \\
0.00 \\
0.04 \\
0.00 \\
0.27 \\
0.26\end{array}$ & $\begin{array}{c}72(0) \\
29.9 \\
0.00 \\
6.25 \\
0.00 \\
0.41 \\
1.83 \\
0.00 \\
0.37 \\
0.87\end{array}$ & $\begin{array}{c}29.9 \\
0.00 \\
6.29 \\
0.00 \\
0.42 \\
1.84 \\
0.00 \\
0.29 \\
0.78\end{array}$ & $\begin{array}{l}29.9 \\
0.00 \\
6.29 \\
0.00 \\
0.37 \\
1.91 \\
0.00 \\
0.31 \\
0.73\end{array}$ \\
\hline \multicolumn{9}{|c|}{$\mathrm{Si} /(\mathrm{Al}+\mathrm{Fe})$} \\
\hline & 1.08 & 1.03 & $\begin{array}{c}(\mathrm{A})+\mathrm{Fe}) \\
1.11\end{array}$ & $\begin{array}{c}2 \mathrm{Mg}+2 \mathrm{C} \\
0.99\end{array}$ & $\begin{array}{c}2 \mathrm{Ba}+\mathrm{Na}+\mathrm{K} \\
0.99\end{array}$ & 1.09 & 1.13 & 1.12 \\
\hline $\begin{array}{l}\mathrm{K} \\
\mathrm{Na} \\
\mathrm{Ca}+\mathrm{Mg}\end{array}$ & $\begin{array}{l}24.6 \\
11.4 \\
64.0\end{array}$ & $\begin{array}{l}22.9 \\
13.8 \\
63.3\end{array}$ & $\begin{array}{c}\text { Mol\% E } \\
21.7 \\
9.2 \\
69.1\end{array}$ & $\begin{array}{c}\text { angeabl } \\
29.2 \\
14.5 \\
56.3\end{array}$ & $\begin{array}{c}\text { Cations } \\
45.7 \\
47.0 \\
7.3\end{array}$ & $\begin{array}{l}25.0 \\
10.5 \\
64.4\end{array}$ & $\begin{array}{r}23.3 \\
8.8 \\
67.9\end{array}$ & $\begin{array}{r}22.1 \\
9.4 \\
68.5\end{array}$ \\
\hline
\end{tabular}




\section{APPENDIX $F$ (cont)}

Drill Hole UE-25a\#1.

\begin{tabular}{|c|c|c|c|c|c|c|c|c|}
\hline $\begin{array}{l}\text { Depth } \\
(\mathrm{ft}) \\
\text { Unit }\end{array}$ & 2220 & 2220 & 2220 & 2220 & $\begin{array}{r}2220 \\
T C p\end{array}$ & 2304 & 2304 & 2304 \\
\hline $\begin{array}{l}\mathrm{SiO} 2 \\
\mathrm{Ti02} \\
\mathrm{A} 1203 \\
\mathrm{Fe} 203 \\
\mathrm{MgO} \\
\mathrm{CaO} \\
\mathrm{BaO} \\
\mathrm{Na} 20 \\
\mathrm{~K} 20\end{array}$ & $\begin{array}{c}66.0 \\
0.00 \\
11.8 \\
0.00 \\
0.56 \\
3.79 \\
0.00 \\
0.34 \\
1.33\end{array}$ & $\begin{array}{c}64.2 \\
0.00 \\
12.1 \\
0.02 \\
0.64 \\
3.87 \\
0.00 \\
0.31 \\
1.48\end{array}$ & $\begin{array}{c}63.3 \\
0.00 \\
11.5 \\
0.03 \\
0.55 \\
3.78 \\
0.00 \\
0.36 \\
1.40\end{array}$ & $\begin{array}{c}62.2 \\
0.00 \\
11.4 \\
0.06 \\
0.55 \\
3.72 \\
0.02 \\
4.35 \\
1.39\end{array}$ & $\begin{array}{c}63.4 \\
0.00 \\
11.4 \\
0.00 \\
0.56 \\
3.80 \\
0.07 \\
0.52 \\
1.42\end{array}$ & $\begin{array}{c}67.7 \\
0.00 \\
11.9 \\
0.00 \\
0.13 \\
4.19 \\
0.20 \\
0.94 \\
1.19\end{array}$ & $\begin{array}{c}67.4 \\
0.00 \\
12.2 \\
0.00 \\
0.15 \\
4.15 \\
0.14 \\
0.89 \\
1.45\end{array}$ & $\begin{array}{c}67.3 \\
0.02 \\
11.3 \\
0.05 \\
0.22 \\
4.20 \\
0.00 \\
0.99 \\
1.68\end{array}$ \\
\hline Total & 83.8 & 82.6 & 81.0 & 79.6 & 81.2 & 86.5 & 86.4 & 85.8 \\
\hline $\begin{array}{l}\mathrm{Si} \\
\mathrm{Ti} \\
\mathrm{Al} \\
\mathrm{Fe} \\
\mathrm{Mg} \\
\mathrm{Ca} \\
\mathrm{Ba} \\
\mathrm{Na} \\
\mathrm{K}\end{array}$ & $\begin{array}{c}29.9 \\
0.00 \\
6.29 \\
0.00 \\
0.38 \\
1.84 \\
0.00 \\
0.30 \\
0.77\end{array}$ & $\begin{array}{c}\text { Uni } \\
29.6 \\
0.00 \\
6.60 \\
0.01 \\
0.44 \\
1.91 \\
0.00 \\
0.28 \\
0.87\end{array}$ & $\begin{array}{c}\text { Ce11 C } \\
29.8 \\
0.00 \\
6.37 \\
0.01 \\
0.39 \\
1.90 \\
0.00 \\
0.33 \\
0.84\end{array}$ & $\begin{array}{c}\text { position } \\
29.7 \\
0.00 \\
6.40 \\
0.02 \\
0.39 \\
1.91 \\
0.00 \\
0.32 \\
0.85\end{array}$ & $\begin{array}{c}\text { Based on } \\
29.8 \\
0.00 \\
6.33 \\
0.00 \\
0.39 \\
1.91 \\
0.01 \\
0.47 \\
0.85\end{array}$ & $\begin{array}{c}72(0) \\
29.9 \\
0.00 \\
6.17 \\
0.00 \\
0 . v 9 \\
1.98 \\
\hat{u} . \bar{v} 3 \\
0.81 \\
0.84\end{array}$ & $\begin{array}{c}29.8 \\
0.00 \\
6.36 \\
0.00 \\
0.10 \\
1.96 \\
0.02 \\
0.76 \\
0.82\end{array}$ & $\begin{array}{c}30.0 \\
0.01 \\
5.95 \\
0.02 \\
0.15 \\
2.00 \\
0.00 \\
0.85 \\
0.95\end{array}$ \\
\hline & 4.76 & 4.48 & 4.57 & $\begin{array}{c}\mathrm{Si} /(\mathrm{A}]+\mathrm{F} \\
4.63\end{array}$ & 4.70 & 4.84 & 4.68 & 5.03 \\
\hline & 1.14 & 1.13 & $\begin{array}{r}(A T+F e) \\
1.11\end{array}$ & $\begin{array}{c}2 \mathrm{Mg}+2 \mathrm{Ca}+ \\
\quad 1.11\end{array}$ & $\begin{array}{c}(\mathrm{Ba}+\mathrm{Na}+\mathrm{K}) \\
1.06\end{array}$ & 1.05 & 1.11 & 0.98 \\
\hline $\begin{array}{l}\mathrm{K} \\
\mathrm{Na} \\
\mathrm{Ca}+\mathrm{Mg}\end{array}$ & $\begin{array}{r}23.4 \\
9.1 \\
67.5\end{array}$ & $\begin{array}{r}24.9 \\
7.9 \\
67.2\end{array}$ & $\begin{array}{c}101 \% \text { EX } \\
24.3 \\
9.5 \\
66.2\end{array}$ & $\begin{array}{c}\text { Ingeabl } \\
24.4 \\
9.4 \\
66.2\end{array}$ & $\begin{array}{c}\text { Cations } \\
23.4 \\
13.0 \\
63.5\end{array}$ & $\begin{array}{l}22.6 \\
21.7 \\
55.7\end{array}$ & $\begin{array}{l}22.4 \\
20.9 \\
56.6\end{array}$ & $\begin{array}{l}24.1 \\
21.6 \\
54.3\end{array}$ \\
\hline
\end{tabular}


Drill Hole UE-25a\#l

\begin{tabular}{|c|c|c|c|c|c|c|c|c|}
\hline $\begin{array}{l}\text { Depth } \\
\text { (ft) } \\
\text { Unit }\end{array}$ & $\begin{array}{r}2304 \\
\text { TCp }\end{array}$ & $\begin{array}{r}2304 \\
T C D\end{array}$ & 2304 & 2304 & $\begin{array}{r}2304 \\
\text { TCD }\end{array}$ & 2304 & 2304 & 2304 \\
\hline $\begin{array}{l}\mathrm{SiO} 02 \\
\mathrm{TiO} 02 \\
\mathrm{~A} 1203 \\
\mathrm{Fe} 2 \mathrm{C3} \\
\mathrm{MgO} \\
\mathrm{CaO} \\
\mathrm{BaO} \\
\mathrm{Na} 20 \\
\mathrm{~K} 20\end{array}$ & $\begin{array}{c}67.4 \\
0.00 \\
11.7 \\
0.03 \\
0.16 \\
4.46 \\
0.02 \\
0.99 \\
1.37\end{array}$ & $\begin{array}{c}64.2 \\
0.02 \\
11.9 \\
0.00 \\
0.19 \\
4.24 \\
0.07 \\
0.76 \\
1.60\end{array}$ & $\begin{array}{c}67.0 \\
0.00 \\
12.1 \\
0.01 \\
0.19 \\
4.31 \\
0.00 \\
0.77 \\
1.62\end{array}$ & $\begin{array}{c}64.9 \\
0.01 \\
12.1 \\
0.03 \\
0.21 \\
4.26 \\
0.00 \\
0.89 \\
1.69\end{array}$ & $\begin{array}{c}64.1 \\
0.01 \\
12.1 \\
0.00 \\
0.15 \\
4.07 \\
0.00 \\
0.78 \\
1.55\end{array}$ & $\begin{array}{c}66.4 \\
0.02 \\
12.5 \\
0.02 \\
0.17 \\
4.25 \\
0.03 \\
0.73 \\
1.64\end{array}$ & $\begin{array}{c}68.8 \\
0.01 \\
11.9 \\
0.07 \\
0.15 \\
4.19 \\
0.00 \\
1.08 \\
1.60\end{array}$ & $\begin{array}{c}68.1 \\
0.03 \\
12.1 \\
0.04 \\
0.17 \\
4.31 \\
0.00 \\
0.89 \\
1.60\end{array}$ \\
\hline Total & 86.2 & 83.0 & 86.9 & 84.1 & 82.7 & 85.7 & 87.8 & 87.3 \\
\hline $\begin{array}{l}\mathrm{Si} \\
\mathrm{Ti} \\
\mathrm{Al} \\
\mathrm{Fe} \\
\mathrm{Mg} \\
\mathrm{Ca} \\
\mathrm{Ba} \\
\mathrm{Na} \\
\mathrm{K}\end{array}$ & $\begin{array}{c}29.9 \\
0.00 \\
6.13 \\
0.01 \\
0.11 \\
2.12 \\
0.00 \\
0.85 \\
0.77\end{array}$ & $\begin{array}{c}\text { Unit } \\
29.6 \\
0.01 \\
6.47 \\
0.00 \\
0.13 \\
2.09 \\
0.01 \\
0.68 \\
0.94\end{array}$ & $\begin{array}{c}\text { Cel l Con } \\
29.8 \\
0.00 \\
6.24 \\
0.00 \\
0.12 \\
2.03 \\
0.00 \\
0.66 \\
0.91\end{array}$ & $\begin{array}{c}\text { sition } \\
29.6 \\
0.00 \\
6.51 \\
0.01 \\
0.14 \\
2.08 \\
0.00 \\
0.79 \\
0.98\end{array}$ & $\begin{array}{c}\text { ased on } \\
29.6 \\
0.00 \\
6.58 \\
0.00 \\
0.10 \\
2.02 \\
0.00 \\
0.70 \\
0.91\end{array}$ & $\begin{array}{c}(0) \\
29.6 \\
0.01 \\
6.57 \\
0.01 \\
0.11 \\
2.03 \\
0.01 \\
0.63 \\
0.93\end{array}$ & $\begin{array}{c}29.9 \\
0.00 \\
6.13 \\
0.02 \\
0.10 \\
1.95 \\
0.00 \\
0.91 \\
0.89\end{array}$ & $\begin{array}{c}29.8 \\
0.01 \\
6.26 \\
0.01 \\
0.11 \\
2.02 \\
0.00 \\
0.75 \\
0.89\end{array}$ \\
\hline \multicolumn{9}{|c|}{$4.78 \begin{array}{c}\mathrm{Si} /(\mathrm{Al}+\mathrm{Fe}) \\
4.53\end{array}$} \\
\hline & 1.01 & \multicolumn{4}{|c|}{$(A l+F e) /(2 M g+2 C a+2 B a+N a+K)$} & 1.12 & 1.04 & 1.06 \\
\hline $\begin{array}{l}\mathrm{K} \\
\mathrm{Na} \\
\mathrm{Ca}+\mathrm{Mg}\end{array}$ & $\begin{array}{l}20.1 \\
22.1 \\
57.8\end{array}$ & $\begin{array}{l}24.5 \\
17.7 \\
57.9\end{array}$ & $\begin{array}{c}\text { Mol\% Ex } \\
24.4 \\
17.6 \\
57.9\end{array}$ & $\begin{array}{r}\text { ngeabl } \\
24.6 \\
19.7 \\
55.7\end{array}$ & $\begin{array}{c}\text { Cations } \\
24.5 \\
18.7 \\
56.8\end{array}$ & $\begin{array}{l}25.2 \\
17.0 \\
57.8\end{array}$ & $\begin{array}{l}23.1 \\
23.7 \\
53.3\end{array}$ & $\begin{array}{l}23.6 \\
20.0 \\
56.4\end{array}$ \\
\hline
\end{tabular}




\section{APPENDIX $F($ cont $)$}

Drill Hole UE-25b\#1(H)

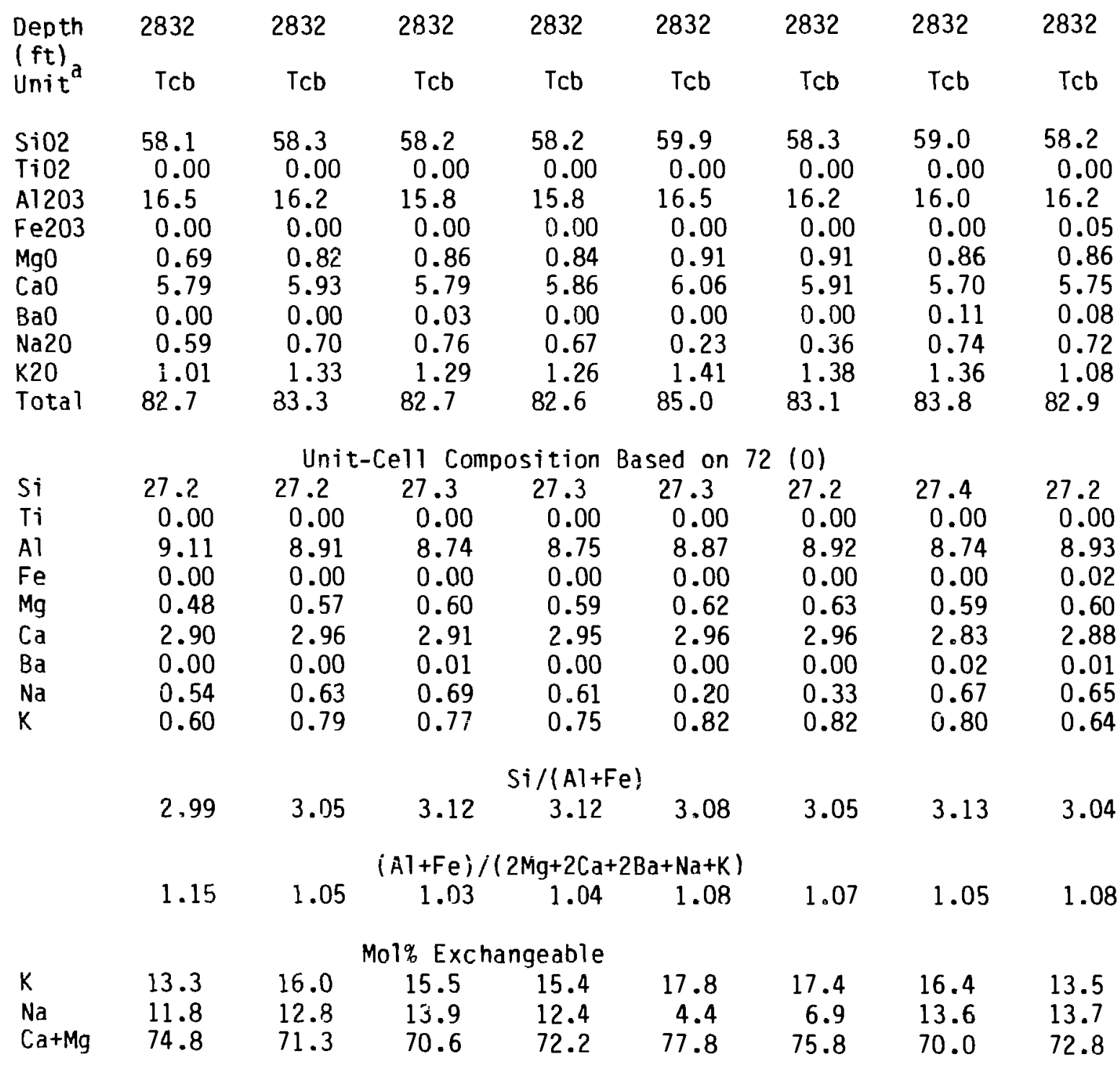


APPENOIX $F$ (cont)

Orill Hole UE-25b\#1(H)

\begin{tabular}{|c|c|c|c|c|c|c|c|c|}
\hline $\begin{array}{l}\text { Depth } \\
(\mathrm{ft}) \\
\text { Unit }\end{array}$ & 2832 & 2832 & 2832 & 2832 & 2832 & 2879 & 2879 & 2879 \\
\hline $\begin{array}{l}\mathrm{SiO} 2 \\
\mathrm{Ti02} \\
\mathrm{Al} 203 \\
\mathrm{Fe} 203 \\
\mathrm{Mgl} \\
\mathrm{Ca} \text { ? } \\
\mathrm{Ba} 5 \\
\mathrm{~N}=20 \\
\mathrm{~K} 2 \mathrm{O}\end{array}$ & $\begin{array}{c}55.5 \\
0.00 \\
16.2 \\
0.00 \\
0.62 \\
5.95 \\
0.15 \\
0.86 \\
1.28\end{array}$ & $\begin{array}{c}57.0 \\
0.03 \\
16.3 \\
0.04 \\
0.67 \\
5.88 \\
0.21 \\
0.59 \\
1.32\end{array}$ & $\begin{array}{c}57.8 \\
0.04 \\
15.1 \\
0.02 \\
0.86 \\
5.92 \\
0.07 \\
0.35 \\
1.30\end{array}$ & $\begin{array}{c}55.8 \\
0.00 \\
15.1 \\
0.05 \\
0.81 \\
5.74 \\
0.17 \\
0.49 \\
1.29\end{array}$ & $\begin{array}{c}56.7 \\
0.00 \\
15.0 \\
0.02 \\
0.79 \\
5.73 \\
0.19 \\
0.50 \\
1.37\end{array}$ & $\begin{array}{c}55.1 \\
0.00 \\
16.3 \\
0.00 \\
0.67 \\
6.07 \\
0.00 \\
0.24 \\
1.54\end{array}$ & $\begin{array}{c}56.5 \\
0.00 \\
16.5 \\
0.00 \\
0.45 \\
6.30 \\
0.00 \\
0.51 \\
1.49\end{array}$ & $\begin{array}{c}54.8 \\
0.00 \\
17.0 \\
0.00 \\
0.34 \\
6.31 \\
0.06 \\
0.66 \\
1.55\end{array}$ \\
\hline Total & 80.5 & 82.1 & 81.5 & 79.5 & 80.3 & 79.9 & 81.7 & 80.8 \\
\hline $\begin{array}{l}\mathrm{Si} \\
\mathrm{Ti} \\
\mathrm{Al} \\
\mathrm{Fe} \\
\mathrm{Mg} \\
\mathrm{Ca} \\
\mathrm{Ba} \\
\mathrm{Na} \\
\mathrm{K}\end{array}$ & $\begin{array}{l}26.9 \\
0.00 \\
9.24 \\
0.00 \\
0.45 \\
3.09 \\
0.03 \\
0.81 \\
0.79\end{array}$ & $\begin{array}{l}\text { Uni } \\
27.0 \\
0.01 \\
9.13 \\
0.01 \\
0.47 \\
2.99 \\
0.04 \\
0.54 \\
0.80\end{array}$ & $\begin{array}{c}\text { e11 Con } \\
27.5 \\
0.01 \\
8.47 \\
0.01 \\
0.61 \\
3.02 \\
0.01 \\
0.32 \\
0.79\end{array}$ & $\begin{array}{l}\text { ition } \\
27.3 \\
0.00 \\
8.73 \\
0.02 \\
0.59 \\
3.01 \\
0.03 \\
0.46 \\
0.81\end{array}$ & $\begin{array}{c}\text { sed on } \\
27.5 \\
0.00 \\
8.56 \\
0.01 \\
0.57 \\
2.97 \\
0.04 \\
0.47 \\
0.85\end{array}$ & $\begin{array}{l}(0) \\
26.8 \\
0.00 \\
9.37 \\
0.00 \\
0.49 \\
3.17 \\
0.00 \\
0.23 \\
0.96\end{array}$ & $\begin{array}{c}26.9 \\
0.00 \\
9.27 \\
0.00 \\
0.32 \\
3.22 \\
0.00 \\
0.47 \\
0.91\end{array}$ & $\begin{array}{l}26.5 \\
0.00 \\
9.73 \\
0.00 \\
0.25 \\
3.27 \\
0.01 \\
0.62 \\
0.96\end{array}$ \\
\hline \multicolumn{9}{|c|}{$\mathrm{Si} /(\mathrm{Al}+\mathrm{Fe})$} \\
\hline \multicolumn{9}{|c|}{$(\mathrm{A}]+\mathrm{Fe}) /(2 \mathrm{Mg}+2 \mathrm{Ca}+2 \mathrm{Ba}+\mathrm{Na}+\mathrm{K})$} \\
\hline \multicolumn{9}{|c|}{ Mol\% Exchangeable Cations } \\
\hline $\begin{array}{l}\mathrm{K} \\
\mathrm{Na} \\
\mathrm{Ca}+\mathrm{Mg}\end{array}$ & $\begin{array}{l}15.4 \\
15.7 \\
68.9\end{array}$ & $\begin{array}{l}16.6 \\
11.3 \\
72.1\end{array}$ & $\begin{array}{r}16.6 \\
6.8 \\
76.5\end{array}$ & $\begin{array}{r}16.5 \\
9.5 \\
73.9\end{array}$ & $\begin{array}{r}17.4 \\
9.7 \\
72.9\end{array}$ & $\begin{array}{r}19.8 \\
4.7 \\
75.5\end{array}$ & $\begin{array}{r}18.4 \\
9.6 \\
72.0\end{array}$ & $\begin{array}{l}18.8 \\
12.2 \\
69.1\end{array}$ \\
\hline
\end{tabular}




\section{APPENDIX $F$ (cont)}

Dri1l Hole UE-25b\#1(H)

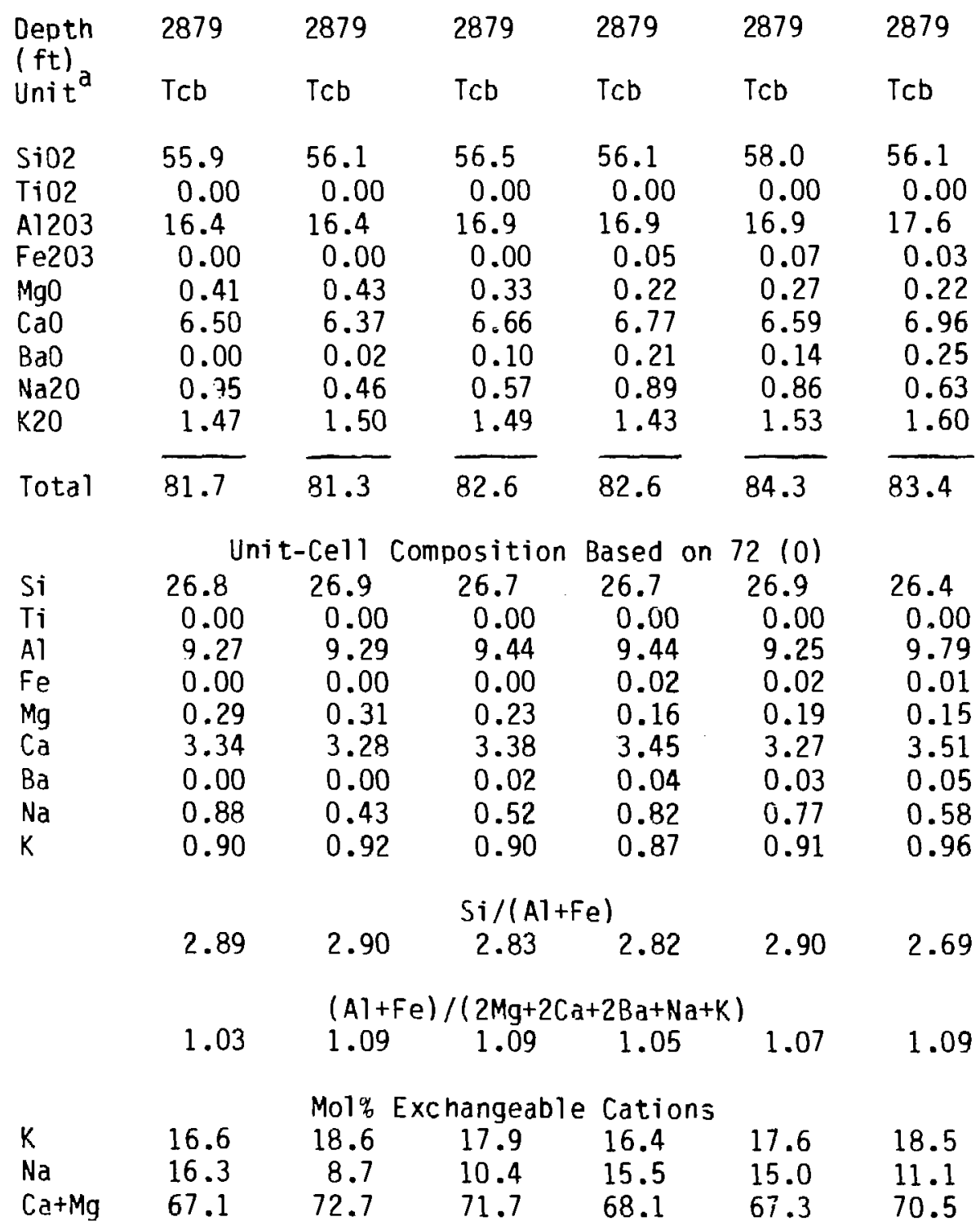


APPENDIX $\vdash$ (cont)

Drill Hole UE-25p\#1

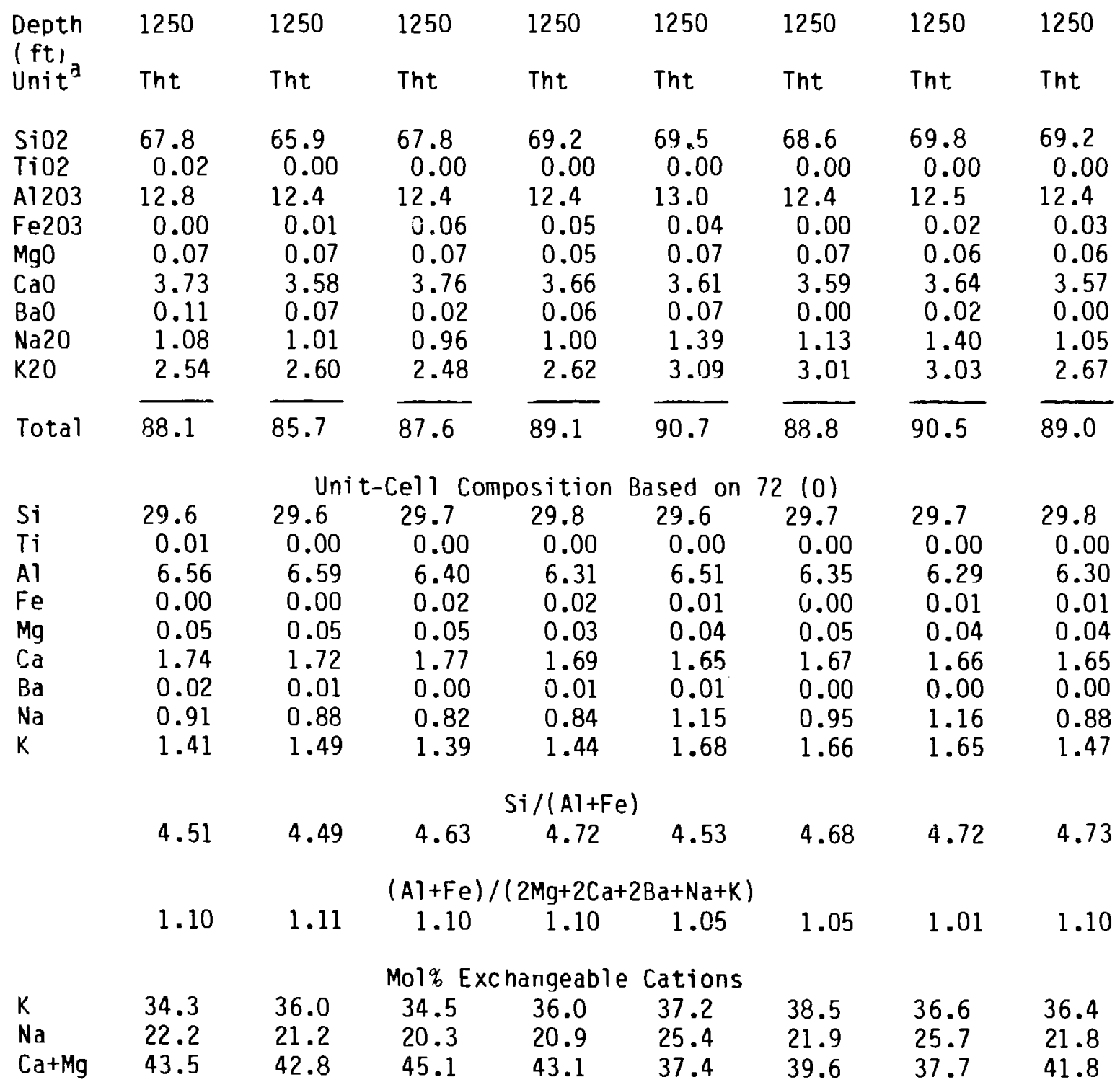




\section{APPENDIX $F$ (cont)}

Drill Hole UE-25p\#1

\begin{tabular}{|c|c|c|c|c|c|c|c|c|}
\hline Depth & 1250 & 1350 & 1350 & 1350 & 1400 & 1400 & 1400 & 1700 \\
\hline Unit ${ }^{a}$ & Tht & Tht & Tht & Tht & Tht & Tht & Tht & TCD \\
\hline $\begin{array}{l}\mathrm{SiO} 02 \\
\mathrm{Ti} 02 \\
\mathrm{Al} 203 \\
\mathrm{Fe} 203 \\
\mathrm{Mg0} \\
\mathrm{CaO} \\
\mathrm{BaO} \\
\mathrm{Na} 20 \\
\mathrm{~K} 20\end{array}$ & $\begin{array}{c}68.9 \\
0.00 \\
12.4 \\
0.00 \\
0.01 \\
3.47 \\
0.04 \\
1.10 \\
2.62\end{array}$ & $\begin{array}{c}69.2 \\
0.00 \\
12.2 \\
0.00 \\
0.08 \\
3.76 \\
0.00 \\
1.11 \\
2.38\end{array}$ & $\begin{array}{c}68.7 \\
0.00 \\
12.5 \\
0.02 \\
0.08 \\
3.87 \\
0.09 \\
0.87 \\
2.27\end{array}$ & $\begin{array}{c}68.3 \\
0.03 \\
11.9 \\
0.00 \\
0.11 \\
3.58 \\
0.13 \\
0.69 \\
2.42\end{array}$ & $\begin{array}{c}66.0 \\
0.00 \\
13.5 \\
0.00 \\
0.07 \\
4.39 \\
0.18 \\
1.23 \\
1.39\end{array}$ & $\begin{array}{c}66.5 \\
0.00 \\
12.9 \\
0.00 \\
0.08 \\
4.08 \\
0.14 \\
1.43 \\
1.52\end{array}$ & $\begin{array}{c}67.4 \\
0.00 \\
12.2 \\
0.00 \\
0.05 \\
3.80 \\
0.12 \\
1.16 \\
1.94\end{array}$ & $\begin{array}{c}64.9 \\
0.00 \\
12.6 \\
0.02 \\
0.24 \\
3.91 \\
0.05 \\
1.33 \\
1.42\end{array}$ \\
\hline Total & 88.6 & 88.7 & 88.4 & 87.2 & 86.8 & 86.6 & 86.7 & 84.5 \\
\hline $\begin{array}{l}\mathrm{Si} \\
\mathrm{Ti} \\
\mathrm{Al} \\
\mathrm{Fe} \\
\mathrm{Mg} \\
\mathrm{Ca} \\
\mathrm{Ba} \\
\mathrm{Na} \\
\mathrm{Ka}\end{array}$ & $\begin{array}{c}29.8 \\
0.00 \\
6.33 \\
0.00 \\
0.01 \\
1.61 \\
0.01 \\
0.92 \\
1.45\end{array}$ & $\begin{array}{c}\text { Uni } \\
29.9 \\
0.00 \\
6.20 \\
0.00 \\
0.05 \\
1.74 \\
0.00 \\
0.93 \\
1.31\end{array}$ & $\begin{array}{c}\text { Cel1 Co } \\
29.8 \\
0.00 \\
6.38 \\
0.01 \\
0.05 \\
1.80 \\
0.02 \\
0.73 \\
1.25\end{array}$ & $\begin{array}{c}\text { osition } \\
30.0 \\
0.01 \\
6.13 \\
0.00 \\
0.07 \\
1.68 \\
0.02 \\
0.59 \\
1.36\end{array}$ & $\begin{array}{c}\text { Based on } \\
29.2 \\
0.00 \\
7.04 \\
0.00 \\
0.05 \\
2.08 \\
0.03 \\
1.05 \\
0.78\end{array}$ & $\begin{array}{c}2(0) \\
29.4 \\
0.00 \\
6.73 \\
0.60 \\
0.05 \\
1.94 \\
0.02 \\
1.23 \\
0.86\end{array}$ & $\begin{array}{c}29.8 \\
0.00 \\
6.37 \\
0.00 \\
0.03 \\
1.80 \\
0.02 \\
0.99 \\
1.09\end{array}$ & $\begin{array}{c}29.4 \\
0.00 \\
6.72 \\
0.01 \\
0.16 \\
1.90 \\
0.01 \\
1.17 \\
0.82\end{array}$ \\
\hline & 4.71 & 4.82 & 4.67 & $\begin{array}{c}\mathrm{i} /(\mathrm{Al}+\mathrm{F} \\
4.89\end{array}$ & 4.15 & 4.37 & 4.67 & 4.37 \\
\hline & 1.13 & 1.06 & $\begin{array}{c}(A 1+F e) \\
1.12\end{array}$ & $\begin{array}{c}2 \mathrm{Mg}+2 \mathrm{C} \\
1.12\end{array}$ & $\begin{array}{c}2 \mathrm{Ba}+\mathrm{Na}+\mathrm{K} \\
1.14\end{array}$ & 1.10 & 1.10 & 1.10 \\
\hline $\begin{array}{l}\mathrm{K} \\
\mathrm{Na} \\
\mathrm{Ca}+\mathrm{Mg}\end{array}$ & $\begin{array}{l}36.3 \\
23.2 \\
40.5\end{array}$ & $\begin{array}{l}32.5 \\
23.1 \\
44.4\end{array}$ & $\begin{array}{l}\text { Mol\% E' } \\
32.7 \\
19.1 \\
48.2\end{array}$ & $\begin{array}{l}\text { angeab1 } \\
36.6 \\
15.9 \\
47.5\end{array}$ & $\begin{array}{c}\text { Cations } \\
19.8 \\
26.6 \\
53.6\end{array}$ & $\begin{array}{l}21.1 \\
30.1 \\
48.8\end{array}$ & $\begin{array}{l}27.9 \\
25.4 \\
46.7\end{array}$ & $\begin{array}{l}20.3 \\
28.9 \\
50.9\end{array}$ \\
\hline
\end{tabular}


APPENDIX $F($ cont)

Drill Hole UE-25p\#1

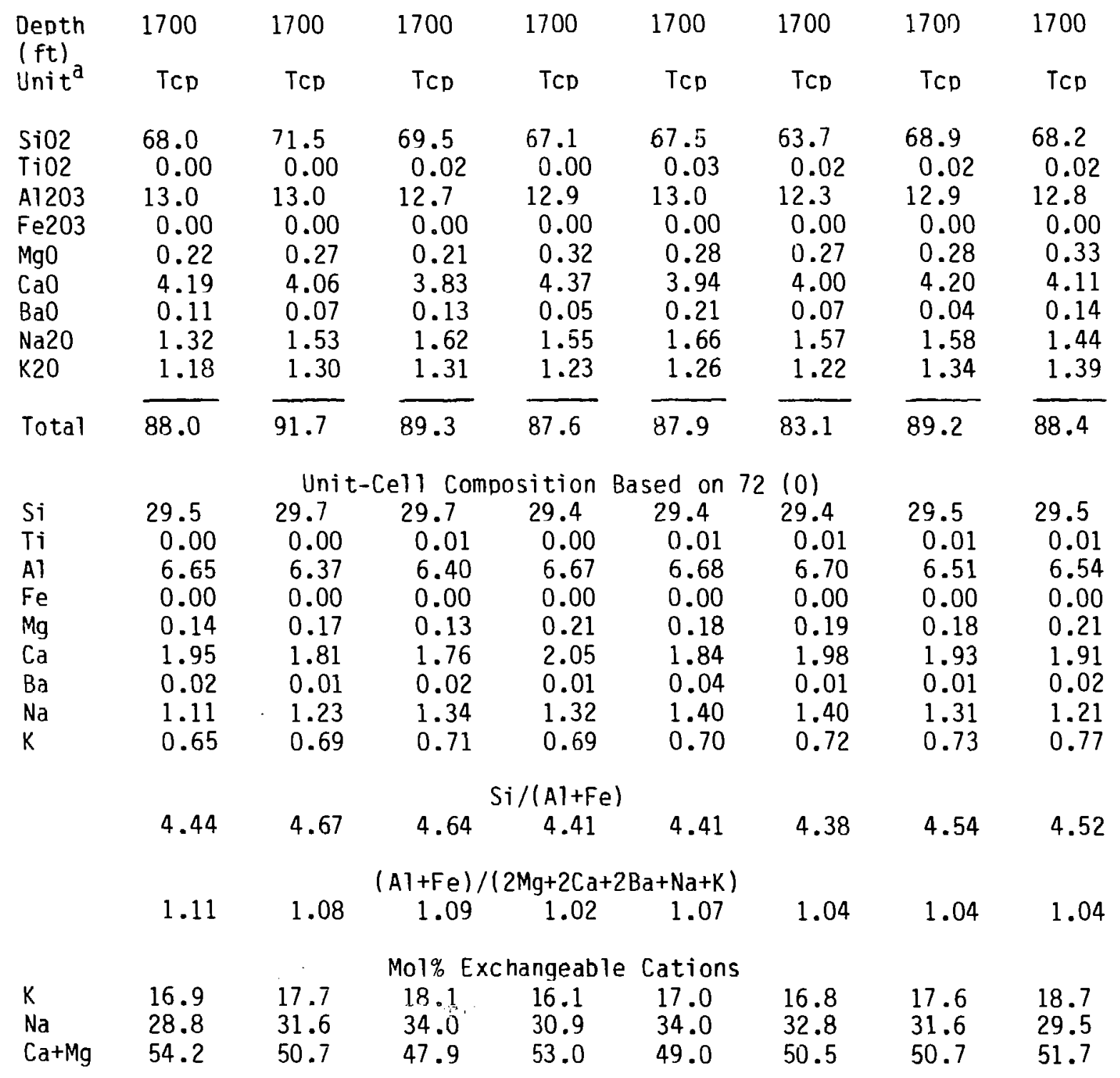




\section{APPENDIX $F$ (cont)}

Dril1 Hole UE-25p\#1

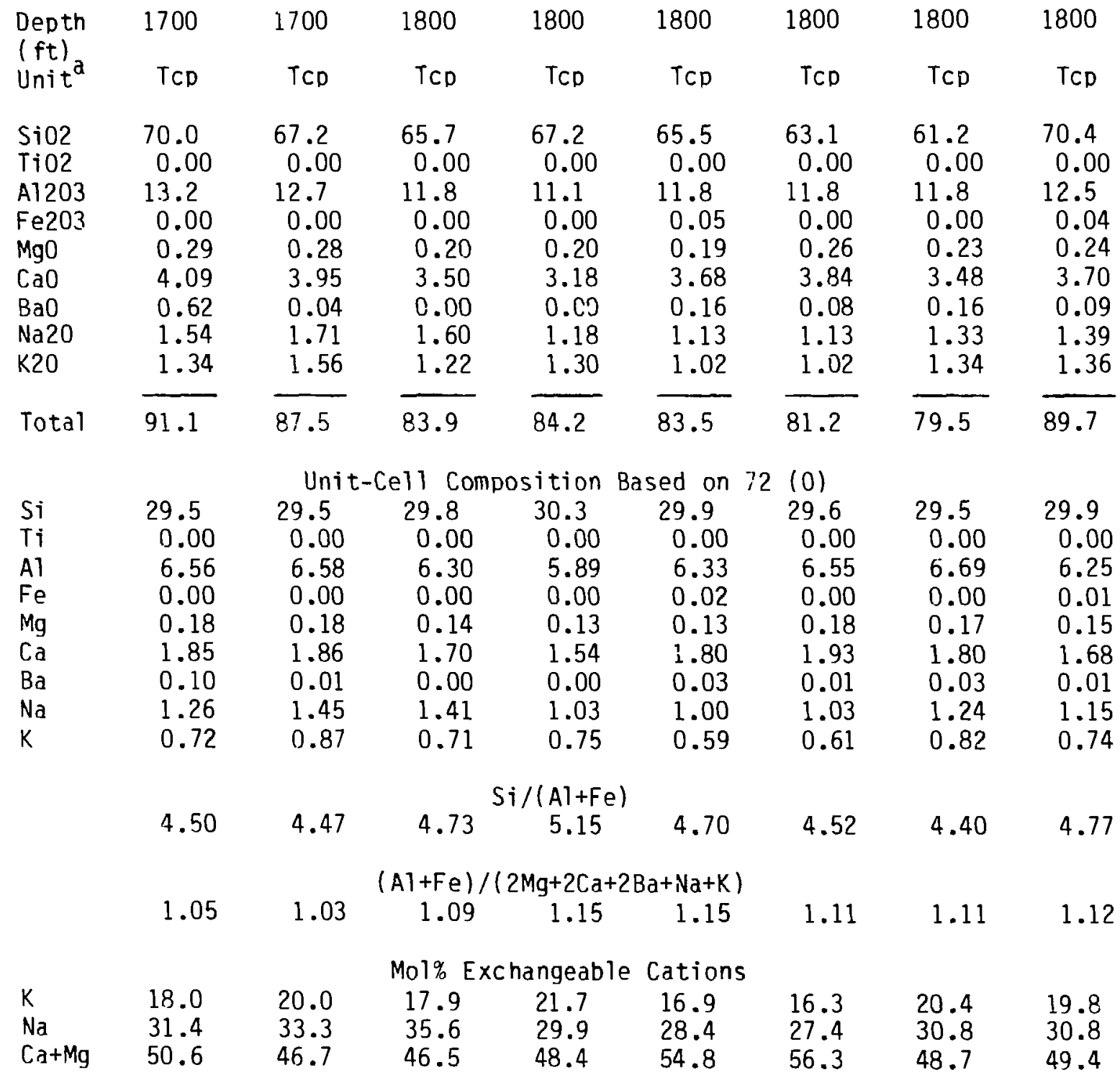


APPENDIX $F$ (cont)

Drill Hole UE-25p\#1

\begin{tabular}{|c|c|c|c|c|c|c|c|c|}
\hline $\begin{array}{l}\text { Depth } \\
(\mathrm{ft}) \\
\text { Unit }\end{array}$ & 1800 & $\begin{array}{r}2760 \\
\text { Tct }\end{array}$ & $\begin{array}{r}2760 \\
\text { Tct }\end{array}$ & $\begin{array}{r}2760 \\
\text { Tct }\end{array}$ & $\begin{array}{r}3330 \\
T 1 r\end{array}$ & $\begin{array}{r}3330 \\
T 1 r\end{array}$ & $\begin{array}{r}3330 \\
T 1 r\end{array}$ & $\begin{array}{r}3330 \\
\operatorname{Tr} r\end{array}$ \\
\hline $\begin{array}{l}\mathrm{SiO} 2 \\
\mathrm{Ti02} \\
\mathrm{Al} 203 \\
\mathrm{Fe} 203 \\
\mathrm{MgO} \\
\mathrm{CaO} \\
\mathrm{BaO} \\
\mathrm{Na} 20 \\
\mathrm{~K} 20\end{array}$ & $\begin{array}{c}68.7 \\
0.00 \\
11.8 \\
0.03 \\
0.23 \\
3.40 \\
0.11 \\
1.38 \\
1.58\end{array}$ & $\begin{array}{c}69.4 \\
0.00 \\
13.4 \\
0.05 \\
0.04 \\
5.30 \\
0.08 \\
0.90 \\
0.51\end{array}$ & $\begin{array}{c}69.1 \\
0.00 \\
13.7 \\
0.00 \\
0.03 \\
5.32 \\
0.15 \\
0.91 \\
0.48\end{array}$ & $\begin{array}{c}68.5 \\
0.00 \\
13.6 \\
0.00 \\
0.00 \\
5.21 \\
0.23 \\
1.13 \\
0.47\end{array}$ & $\begin{array}{c}60.3 \\
0.02 \\
15.0 \\
0.00 \\
0.12 \\
7.12 \\
0.32 \\
0.76 \\
0.28\end{array}$ & $\begin{array}{c}60.6 \\
0.00 \\
16.0 \\
0.00 \\
0.08 \\
7.22 \\
0.19 \\
0.85 \\
0.41\end{array}$ & $\begin{array}{c}63.5 \\
0.00 \\
15.5 \\
0.00 \\
0.06 \\
6.76 \\
0.22 \\
1.02 \\
0.43\end{array}$ & $\begin{array}{c}60.4 \\
0.01 \\
15.1 \\
0.00 \\
0.08 \\
6.57 \\
0.07 \\
1.34 \\
0.38\end{array}$ \\
\hline Total & 87.3 & 89.8 & 89.7 & 89.1 & 83.9 & 85.4 & 87.5 & 84.0 \\
\hline $\begin{array}{l}\mathrm{Si} \\
\mathrm{Ti} \\
\mathrm{Al} \\
\mathrm{Fe} \\
\mathrm{Mg} \\
\mathrm{Ca} \\
\mathrm{Ba} \\
\mathrm{Na} \\
\mathrm{K}\end{array}$ & $\begin{array}{c}30.0 \\
0.00 \\
6.09 \\
0.01 \\
0.15 \\
1.59 \\
0.02 \\
1.17 \\
0.88\end{array}$ & $\begin{array}{c}\text { Un } \\
29.5 \\
0.00 \\
6.72 \\
0.02 \\
0.03 \\
2.41 \\
0.01 \\
0.74 \\
0.28\end{array}$ & $\begin{array}{c}\text { Cel1 Co } \\
29.4 \\
0.00 \\
6.86 \\
0.00 \\
0.02 \\
2.42 \\
0.02 \\
0.75 \\
0.26\end{array}$ & $\begin{array}{c}\text { position } \\
29.4 \\
0.00 \\
6.85 \\
0.00 \\
0.00 \\
2.39 \\
0.04 \\
0.94 \\
0.26\end{array}$ & $\begin{array}{c}\text { Based on } \\
27.8 \\
0.01 \\
8.14 \\
0.00 \\
0.08 \\
3.52 \\
0.06 \\
0.68 \\
0.16\end{array}$ & $\begin{array}{c}72(0) \\
27.5 \\
0.00 \\
8.59 \\
0.00 \\
0.05 \\
3.51 \\
0.03 \\
0.75 \\
0.24\end{array}$ & $\begin{array}{c}28.0 \\
0.00 \\
8.07 \\
0.00 \\
0.04 \\
3.20 \\
0.04 \\
0.87 \\
0.24\end{array}$ & $\begin{array}{c}27.8 \\
0.00 \\
8.22 \\
0.00 \\
0.05 \\
3.24 \\
0.01 \\
1.20 \\
0.22\end{array}$ \\
\hline \multirow{2}{*}{ K } & 4.92 & 4.37 & 4.28 & $\begin{array}{c}\mathrm{Si} /(\mathrm{A}]+\mathrm{F} \\
4.29\end{array}$ & 3.42 & 3.20 & 3.47 & 3.38 \\
\hline & 1.10 & 1.14 & $\begin{array}{c}(A]+F e) / \\
1.15\end{array}$ & $\begin{array}{c}2 \mathrm{Mg}+2 \mathrm{Ca}+ \\
1.13\end{array}$ & $\begin{array}{c}(\mathrm{Ba}+\mathrm{Na}+\mathrm{K}) \\
1.00\end{array}$ & 1.05 & 1.05 & 1.02 \\
\hline $\begin{array}{l}\mathrm{K} \\
\mathrm{Na} \\
\mathrm{Ca}+\mathrm{Mg}\end{array}$ & $\begin{array}{l}23.2 \\
30.8 \\
45.9\end{array}$ & $\begin{array}{r}8.0 \\
21.5 \\
70.5\end{array}$ & $\begin{array}{c}\text { Mol\% E } \\
7.5 \\
21.7 \\
70.7\end{array}$ & $\begin{array}{c}\text { nangeab } \\
7.2 \\
26.2 \\
66.7\end{array}$ & $\begin{array}{c}\text { Cations } \\
3.7 \\
15.3 \\
81.0\end{array}$ & $\begin{array}{r}5.2 \\
16.4 \\
78.3\end{array}$ & $\begin{array}{r}5.6 \\
20.1 \\
74.4\end{array}$ & $\begin{array}{r}4.7 \\
25.4 \\
69.9\end{array}$ \\
\hline
\end{tabular}




\section{APPENDIX $F$ (cont)}

Drill Hole UE-25p\#1

$\begin{array}{lcccccccc}\begin{array}{l}\text { Depth } \\ \text { (ft) }\end{array} & 3330 & 3330 & 3330 & 3330 & 3330 & 3330 & 3330 & 3330 \\ \text { Unit } & \text { T1r } & \text { T1r } & \text { T1r } & \text { T1r } & \text { T1r } & \text { T1r } & \text { T1r } & \text { TIr } \\ \text { Si02 } & 63.3 & 63.3 & 63.2 & 63.0 & 63.4 & 64.7 & 62.8 & 64.1 \\ \text { Ti02 } & 0.00 & 0.00 & 0.00 & 0.00 & 0.00 & 0.00 & 0.04 & 0.00 \\ \text { A1203 } & 15.3 & 15.0 & 14.9 & 14.9 & 15.5 & 15.3 & 15.2 & 15.5 \\ \text { Fe203 } & 0.00 & 0.00 & 0.00 & 0.02 & 0.04 & 0.05 & 0.00 & 0.02 \\ \text { Mg0 } & 0.08 & 0.06 & 0.08 & 0.06 & 0.15 & 0.12 & 0.19 & 0.10 \\ \text { Ca0 } & 6.58 & 6.84 & 6.47 & 6.59 & 6.66 & 6.83 & 6.82 & 6.60 \\ \text { Ba0 } & 0.08 & 0.16 & 0.27 & 0.14 & 0.05 & 0.00 & 0.16 & 0.00 \\ \text { Na20 } & 1.03 & 0.85 & 0.99 & 1.03 & 0.92 & 0.67 & 0.73 & 1.31 \\ \text { K20 } & 0.48 & 0.42 & 0.48 & 0.47 & 0.51 & 0.47 & 0.47 & 0.51 \\ \text { Total } & \mathbf{8 6 . 9} & \underline{86.7} & \underline{86.4} & \underline{86.1} & \underline{87.2} & \underline{88.1} & \frac{86.4}{88.1}\end{array}$

Si

Unit-Cell Composition Based on $72(0)$

Ti

28.1

28.2

28.2

28.2

28.0

28.2

28.0

28.1

A1

0.00

0.00

0.00

0.00

0.00

0.00

0.01

0.00

$\mathrm{Fe}$

0.00

0.00

0.87

7.86

8.07

7.87

8.01

7.98

$\mathrm{Mg}$

0.05

0.04

0.05

0.01

0.01

0.02

0.00

0.01

3.13

3.10

0.04

0.10

0.08

0.13

0.07

$\mathrm{Ba}$

3.26

0.05

3.16

3.16

3.20

3.27

3.10

0.89

0.73

0.86

0.02

0.01

0.00

0.03

0.00

$\mathrm{K}$

0.27

0.27

0.89

0.79

0.57

0.63

1.11

0.29

0.26

0.27

0.28

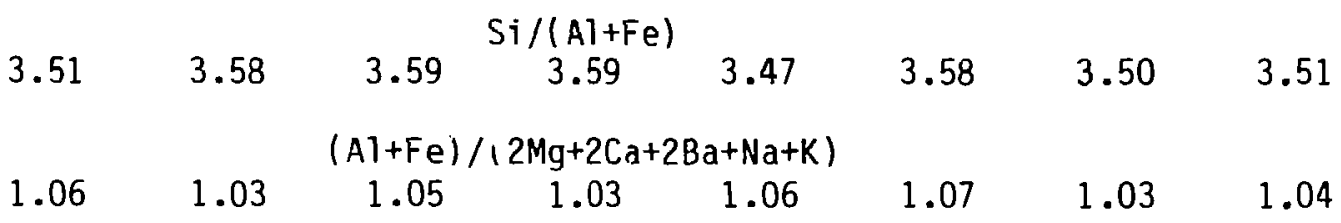

Mol\% Exchangeable Cations

$\begin{array}{lrrrrrrrr}\mathrm{K} & 6.3 & 5.6 & 6.4 & 6.2 & 6.6 & 6.4 & 6.2 & 6.2 \\ \mathrm{Na} & 20.4 & 17.2 & 20.0 & 20.5 & 18.2 & 13.8 & 14.7 & 24.4 \\ \mathrm{Ca}+\mathrm{Mg} & 73.3 & 77.3 & 73.6 & 73.4 & 75.1 & 79.8 & 79.0 & 69.4\end{array}$




\section{APPENDIX $F$ (cont)}

Drill Hole J-13

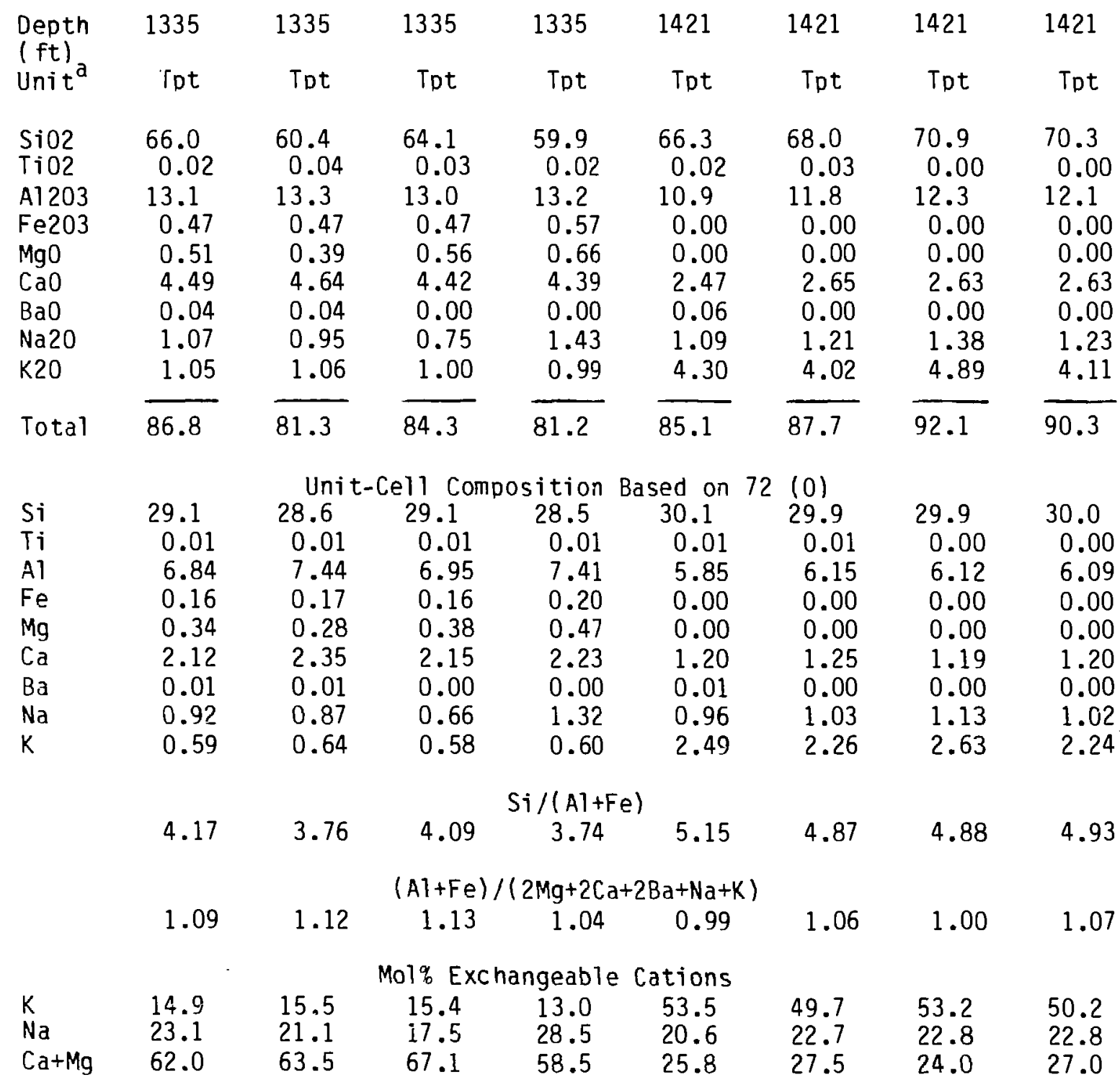


APPENDIX $F$ (cont)

Drill Hole J-13

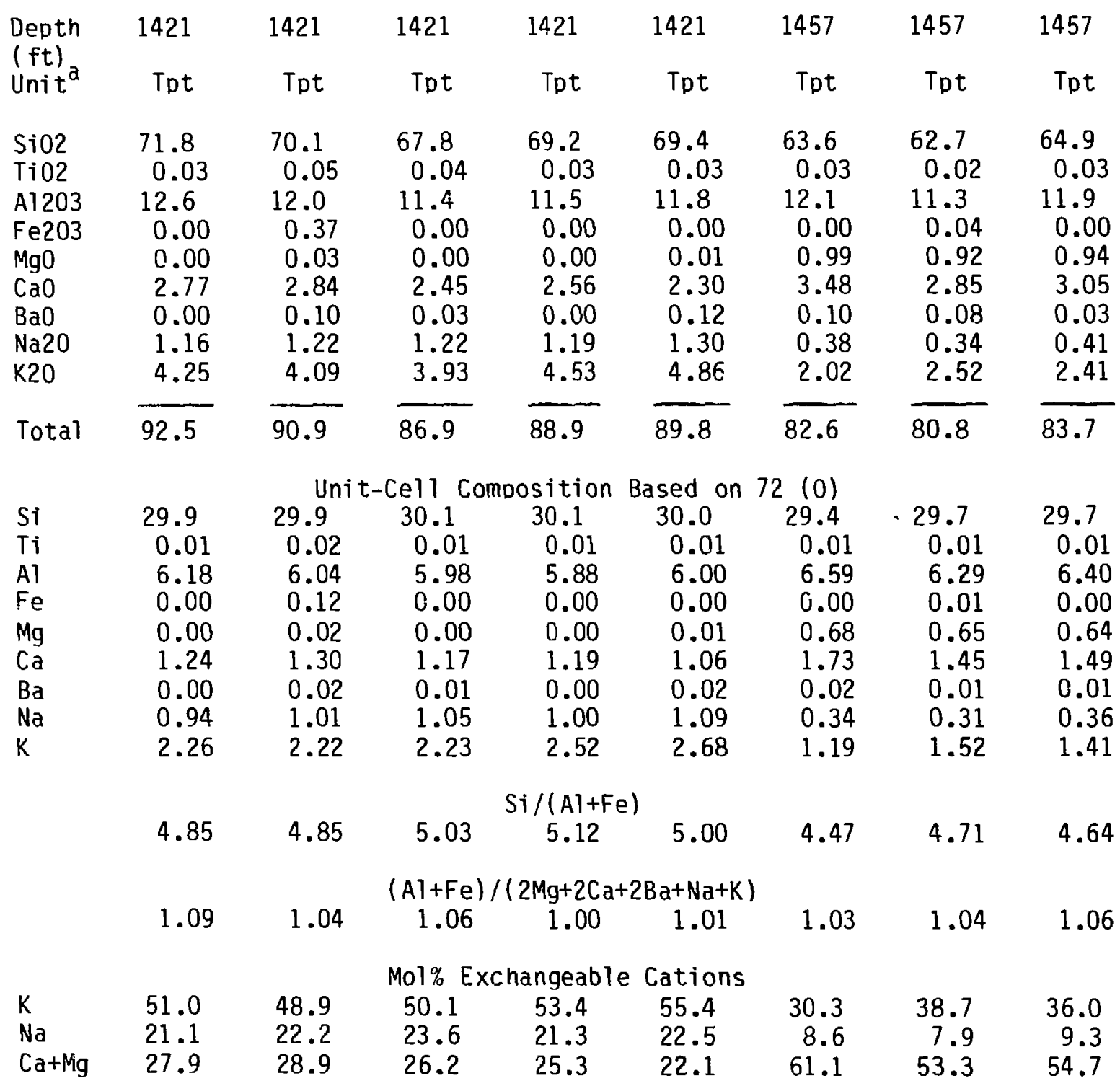


APPENDIX F (cont)

Drill Hole J-13

$\begin{array}{lcccccccc}\begin{array}{l}\text { Depth } \\ \text { (ft) }\end{array} & 1457 & 1457 & 1457 & 1457 & 1457 & 1457 & 1519 & 1519 \\ \text { Unit } & \text { Tpt } & \text { Tpt } & \text { Tpt } & \text { Tpt } & \text { Tpt } & \text { Tpt } & \text { Tht } & \text { Tht } \\ \text { Si02 } & 65.4 & 64.1 & 65.3 & 65.4 & 65.5 & 63.9 & 66.1 & 65.5 \\ \text { Ti02 } & 0.03 & 0.00 & 0.00 & 0.00 & 0.00 & 0.03 & 0.02 & 0.02 \\ \text { A1203 } & 11.9 & 11.7 & 11.5 & 11.9 & 11.8 & 11.7 & 11.9 & 12.2 \\ \text { Fe203 } & 0.00 & 0.06 & 0.08 & 0.18 & 0.04 & 0.16 & 0.00 & 0.03 \\ \text { Mg0 } & 0.89 & 0.92 & 0.93 & 0.88 & 0.94 & 0.04 & 0.40 & 0.51 \\ \text { Ca0 } & 3.07 & 2.95 & 2.76 & 3.10 & 3.01 & 2.83 & 3.50 & 3.22 \\ \text { Ba0 } & 0.00 & 0.05 & 0.10 & 0.16 & 0.09 & 0.19 & 0.00 & 0.00 \\ \text { Na20 } & 0.49 & 0.46 & 0.39 & 0.50 & 0.59 & 0.31 & 0.53 & 0.66 \\ \text { K20 } & 2.62 & 2.52 & 2.57 & 2.58 & 2.88 & 2.42 & \frac{2.85}{3.10} & \frac{3.10}{85.3} \\ \text { Tota1 } & \frac{84.4}{82.8} & \frac{83.7}{84.7} & \frac{84.8}{82.5} & \frac{82.5}{85.3} & \frac{85.3}{34}\end{array}$

Unit-Cell Composition Based on $72(0)$

\begin{tabular}{|c|c|c|c|c|c|c|c|c|}
\hline Si & 29.7 & 29.7 & 29.9 & 29.7 & 29.7 & 29.7 & 29.8 & 29.6 \\
\hline Ti & 0.01 & 0.00 & 0.00 & 0.00 & 0.00 & 0.01 & 0.01 & 0.01 \\
\hline Al & 6.36 & 6.37 & 6.22 & 6.34 & 6.28 & 6.42 & 6.30 & 6.51 \\
\hline $\mathrm{Fe}$ & 0.00 & 0.02 & 0.03 & 0.06 & 0.01 & 0.06 & 0.00 & 0.01 \\
\hline $\mathrm{Mg}$ & 0.60 & 0.63 & 0.63 & 0.59 & 0.63 & 0.65 & 0.27 & 0.34 \\
\hline $\mathrm{Ca}$ & 1.49 & 1.46 & 1.35 & 1.51 & 1.46 & 1.41 & 1.69 & 1.56 \\
\hline $\mathrm{Ba}$ & 0.00 & 0.01 & 0.02 & 0.03 & 0.02 & 0.03 & 0.00 & 0.00 \\
\hline $\mathrm{Na}$ & 0.43 & 0.41 & 0.35 & 0.44 & 0.52 & 0.28 & 0.46 & 0.58 \\
\hline K & 1.52 & 1.49 & 1.50 & 1.49 & 1.66 & 1.43 & 1.64 & 1.79 \\
\hline \multicolumn{9}{|c|}{$\mathrm{Si} /(A \mathrm{Al}+\mathrm{Fe})$} \\
\hline & 4.67 & 4.64 & 4.78 & 4.64 & 4.72 & 4.58 & 4.72 & 4.53 \\
\hline \multirow{2}{*}{\multicolumn{9}{|c|}{$\begin{array}{c}(\mathrm{Al}+\mathrm{Fe}) /(2 \mathrm{Mg}+2 \mathrm{Ca}+2 \mathrm{Ba}+\mathrm{Na}+\mathrm{K}) \\
1.07 \quad 1.03 \quad 0.98\end{array}$}} \\
\hline & 1.04 & 1.05 & 1.07 & 1.03 & 0.98 & 1.10 & 1.05 & 1.06 \\
\hline \multicolumn{9}{|c|}{ Mol\% Exchangeabl } \\
\hline K & 37.5 & 37.2 & 39.1 & 37.0 & 38.9 & 38.0 & 40.4 & 41.9 \\
\hline $\mathrm{Na}$ & 10.7 & 10.3 & 9.0 & 10.9 & 12.1 & 7.4 & 11.4 & 13.5 \\
\hline $\mathrm{Ca}+\mathrm{Mg}$ & 51.8 & 52.5 & 51.8 & 52.1 & 49.0 & 54.6 & 48.2 & 44.6 \\
\hline
\end{tabular}


APPENDIX $F$ (cont)

Drill Hole J-13

\begin{tabular}{|c|c|c|c|c|c|c|c|c|}
\hline $\begin{array}{l}\text { Depth } \\
(\mathrm{ft}) \\
\text { Unit }\end{array}$ & 1519 & $\begin{array}{r}1519 \\
\text { Tht }\end{array}$ & $\begin{array}{r}1519 \\
\text { Tht }\end{array}$ & 1519 & 1519 & 1519 & 1519 & 1519 \\
\hline $\begin{array}{l}\mathrm{SiO} 02 \\
\mathrm{Ti02} \\
\mathrm{A} 1203 \\
\mathrm{Fe} 203 \\
\mathrm{Mg0} \\
\mathrm{CaO} \\
\mathrm{BaO} \\
\mathrm{Na} 20 \\
\mathrm{~K} 20\end{array}$ & $\begin{array}{c}65.9 \\
0.00 \\
12.6 \\
0.00 \\
0.53 \\
3.79 \\
0.08 \\
0.52 \\
2.71\end{array}$ & $\begin{array}{c}64.0 \\
0.03 \\
12.2 \\
0.02 \\
0.38 \\
3.82 \\
0.10 \\
0.63 \\
2.40\end{array}$ & $\begin{array}{c}64.0 \\
0.03 \\
12.3 \\
0.08 \\
0.47 \\
3.74 \\
0.00 \\
0.77 \\
3.19\end{array}$ & $\begin{array}{c}66.0 \\
0.02 \\
12.1 \\
0.07 \\
0.50 \\
3.59 \\
0.07 \\
0.56 \\
2.91\end{array}$ & $\begin{array}{c}66.8 \\
0.00 \\
12.5 \\
0.01 \\
0.45 \\
3.49 \\
0.00 \\
0.72 \\
3.35\end{array}$ & $\begin{array}{c}66.4 \\
0.02 \\
12.7 \\
0.02 \\
0.47 \\
3.63 \\
0.00 \\
0.75 \\
3.22\end{array}$ & $\begin{array}{c}66.8 \\
0.03 \\
12.7 \\
0.04 \\
0.52 \\
3.66 \\
0.00 \\
0.71 \\
3.60\end{array}$ & $\begin{array}{c}67.6 \\
0.00 \\
11.8 \\
0.00 \\
0.42 \\
3.42 \\
0.00 \\
0.63 \\
3.27\end{array}$ \\
\hline Total & 86.1 & 83.5 & 84.6 & 85.9 & 87.3 & 87.2 & 88.0 & 87.2 \\
\hline $\begin{array}{l}\mathrm{Si} \\
\mathrm{Ti} \\
\mathrm{Al} \\
\mathrm{Fe} \\
\mathrm{Mg} \\
\mathrm{Ca} \\
\mathrm{Ba} \\
\mathrm{Na} \\
\mathrm{K}\end{array}$ & $\begin{array}{c}29.4 \\
0.00 \\
6.65 \\
0.00 \\
0.35 \\
1.81 \\
0.01 \\
0.45 \\
1.54\end{array}$ & $\begin{array}{c}\text { Uni } \\
29.4 \\
0.01 \\
6.62 \\
0.01 \\
0.26 \\
1.88 \\
0.02 \\
0.56 \\
1.41\end{array}$ & $\begin{array}{c}\text { Cel1 Co Co } \\
29.3 \\
0.01 \\
6.62 \\
0.03 \\
0.32 \\
1.83 \\
0.00 \\
0.68 \\
1.86\end{array}$ & $\begin{array}{c}\text { osition } \\
29.6 \\
0.01 \\
6.42 \\
0.02 \\
0.33 \\
1.72 \\
0.01 \\
0.49 \\
1.66\end{array}$ & $\begin{array}{c}\text { 3ased on } \\
29.5 \\
0.00 \\
6.52 \\
0.00 \\
0.30 \\
1.65 \\
0.00 \\
0.62 \\
1.89\end{array}$ & $\begin{array}{r}72(0) \\
29.4 \\
0.01 \\
6.62 \\
0.01 \\
0.31 \\
1.72 \\
0.00 \\
0.64 \\
1.82\end{array}$ & $\begin{array}{c}29.3 \\
0.01 \\
6.59 \\
0.01 \\
0.34 \\
1.72 \\
0.00 \\
0.61 \\
2.02\end{array}$ & $\begin{array}{c}29.8 \\
0.00 \\
6.15 \\
0.00 \\
0.28 \\
1.62 \\
0.00 \\
0.54 \\
1.84\end{array}$ \\
\hline \multicolumn{9}{|c|}{$\mathrm{Si} /(\mathrm{A})+\mathrm{Fe})$} \\
\hline \multicolumn{7}{|c|}{$(\mathrm{A}]+\mathrm{Fe}) /(2 \mathrm{Mg}+2 \mathrm{Ca}+2 \mathrm{Ba}+\mathrm{Na}+\mathrm{K})$} & 0.98 & 1.00 \\
\hline $\begin{array}{l}\mathrm{K} \\
\mathrm{Na} \\
\mathrm{Ca}+\mathrm{Mg}\end{array}$ & $\begin{array}{l}37.1 \\
10.8 \\
52.1\end{array}$ & $\begin{array}{l}34.2 \\
13.7 \\
52.1\end{array}$ & $\begin{array}{c}\text { Mol\% E } \\
39.6 \\
14.5 \\
45.8\end{array}$ & $\begin{array}{c}\text { angeab } \\
39.5 \\
11.6 \\
48.9\end{array}$ & $\begin{array}{c}\text { Cations } \\
42.4 \\
13.8 \\
43.8\end{array}$ & $\begin{array}{l}40.5 \\
14.3 \\
45.2\end{array}$ & $\begin{array}{l}43.1 \\
12.9 \\
44.0\end{array}$ & $\begin{array}{l}43.1 \\
12.6 \\
44.3\end{array}$ \\
\hline
\end{tabular}


APPENDIX $F$ (cont)

Drill Hole J-13

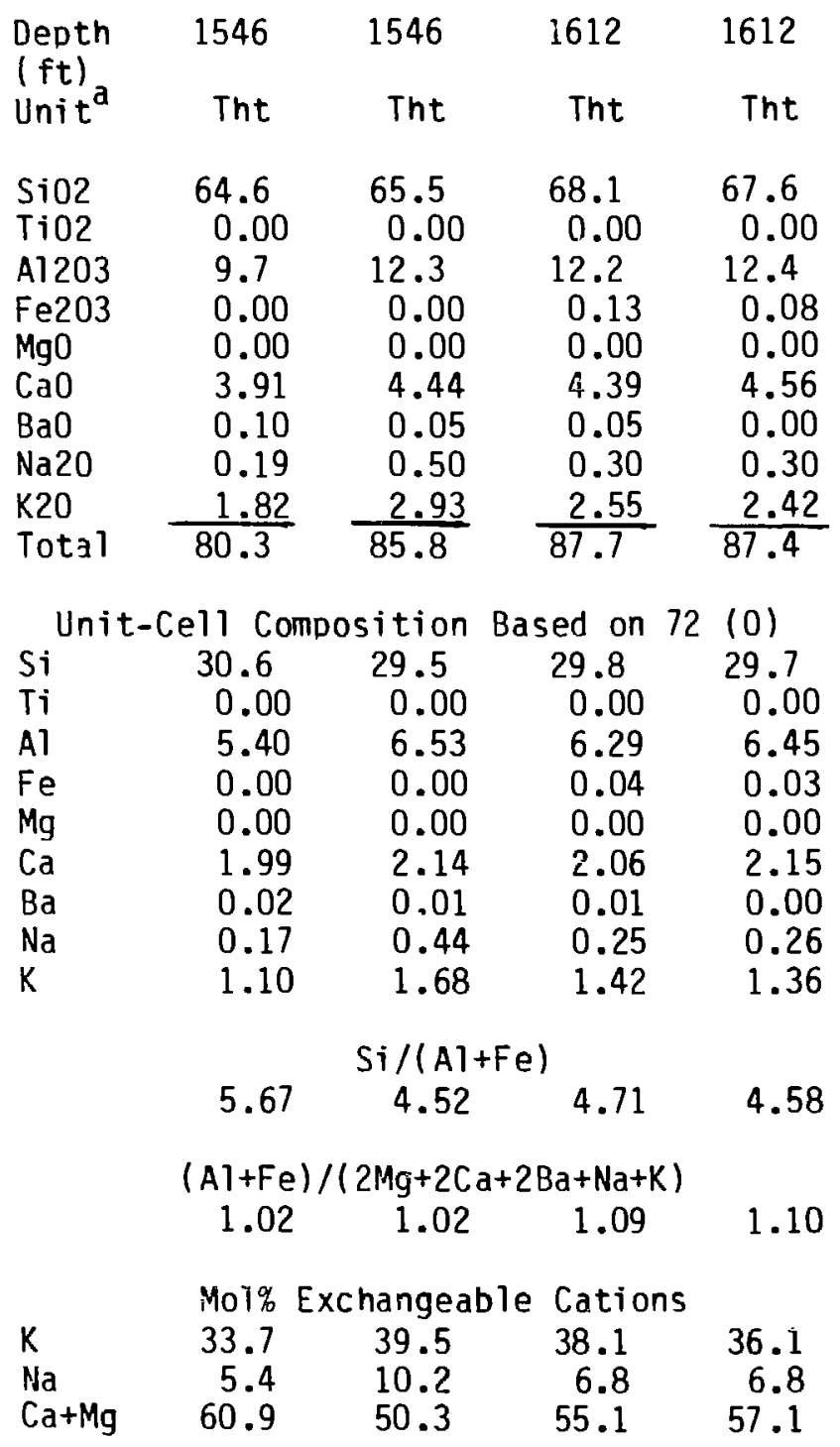




\section{APPENDIX $F($ cont $)$}

Outcrop at Prow Pass, Northern Yucca Mountain

Sample Number: 3-15-82-11; Nevada State Coordinates (ft) 786550N, 551400E

Partially welded tuff near hise of Topopah Spring Member, Paintbrush Tuff

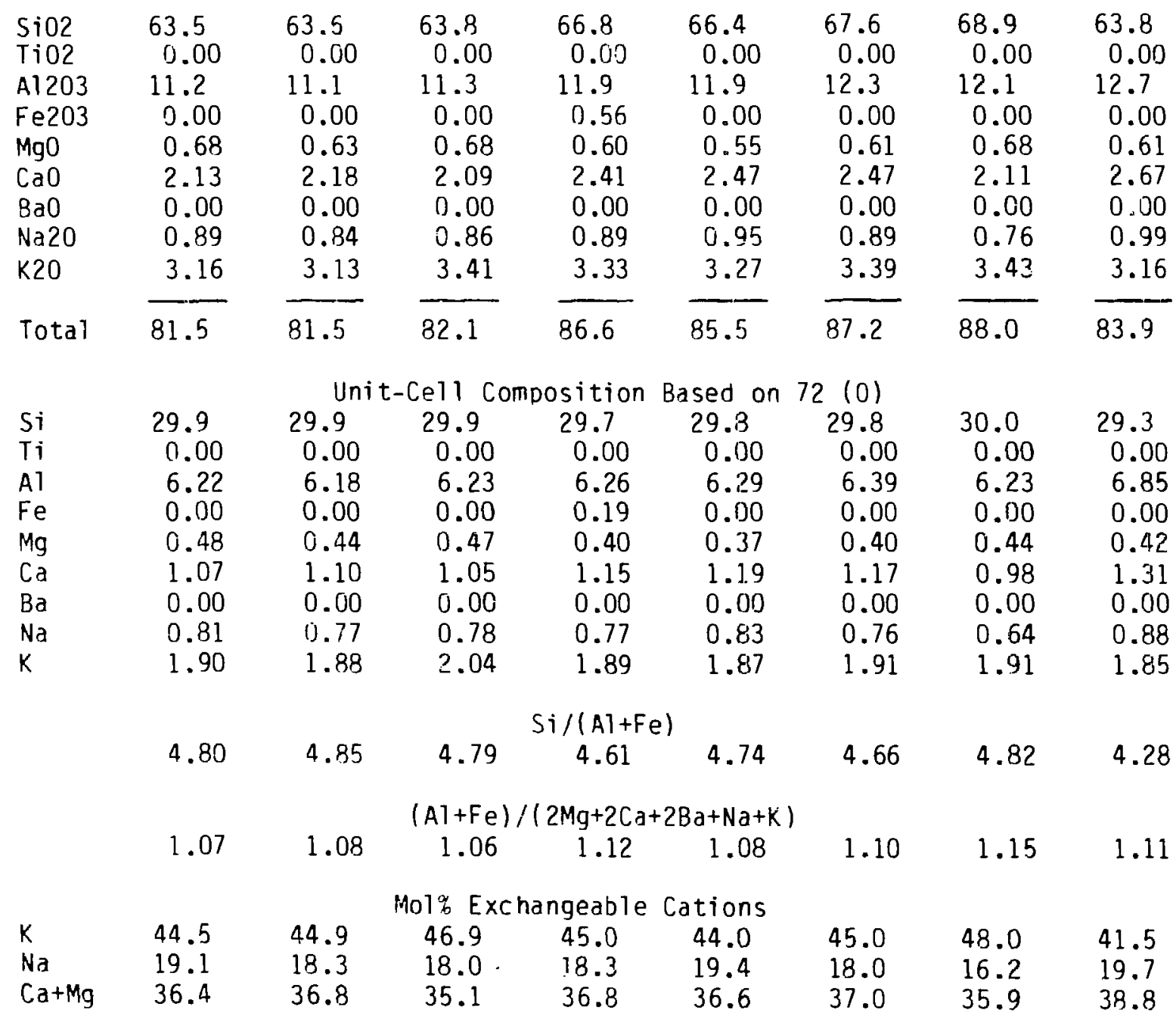




\section{APPENDIX $F$ (cont)}

Outcrop at Prow Pass, Northern Yucca Mountain

Sample

Number

$5-6-81-1$

$3-15-82-6$

Nonwelded zeolitic tuff in upper and central part of tuff of Calico Hills.

Nevada State Coordinates ( ft) for sample locations 5-6-86-1=782850N, 550625E; $3-15-82-6=786400 \mathrm{~N}, 551100 \mathrm{E}$.

\begin{tabular}{|c|c|c|c|c|c|c|c|c|}
\hline $\begin{array}{l}\mathrm{SiO} 2 \\
\mathrm{TiO} 2 \\
\mathrm{~A} 1203 \\
\mathrm{Fe} 203 \\
\mathrm{MgO} \\
\mathrm{CaO} \\
\mathrm{BaO} \\
\mathrm{Na2O} \\
\mathrm{K} 20\end{array}$ & $\begin{array}{c}63.3 \\
0.00 \\
12.6 \\
0.00 \\
0.74 \\
2.77 \\
0.00 \\
0.34 \\
3.80\end{array}$ & $\begin{array}{c}63.4 \\
0.00 \\
12.4 \\
0.00 \\
0.81 \\
2.69 \\
0.20 \\
0.29 \\
3.68\end{array}$ & $\begin{array}{c}67.3 \\
0.00 \\
11.9 \\
0.00 \\
0.38 \\
2.37 \\
0.00 \\
0.52 \\
5.27\end{array}$ & $\begin{array}{c}65.5 \\
0.00 \\
12.8 \\
0.00 \\
0.81 \\
2.55 \\
0.00 \\
0.43 \\
4.14\end{array}$ & $\begin{array}{c}64.7 \\
0.00 \\
12.7 \\
0.00 \\
1.13 \\
2.37 \\
0.00 \\
0.35 \\
3.77\end{array}$ & $\begin{array}{c}64.4 \\
0.00 \\
12.6 \\
0.00 \\
0.76 \\
2.82 \\
0.00 \\
0.43 \\
3.99\end{array}$ & $\begin{array}{c}65.3 \\
0.00 \\
12.9 \\
0.00 \\
0.70 \\
2.76 \\
0.00 \\
0.38 \\
3.94\end{array}$ & $\begin{array}{c}64.0 \\
0.00 \\
12.3 \\
0.00 \\
0.50 \\
2.33 \\
0.00 \\
0.36 \\
5.58\end{array}$ \\
\hline Total & 83.5 & 83.4 & 87.7 & 86.2 & 85.0 & 85.0 & 86.0 & 85.1 \\
\hline $\begin{array}{l}\mathrm{Si} \\
\mathrm{Ti} \\
\mathrm{Al} \\
\mathrm{Fe} \\
\mathrm{Mg} \\
\mathrm{Ca} \\
\mathrm{Ba} \\
\mathrm{Na}\end{array}$ & $\begin{array}{l}29.3 \\
0.00 \\
6.88 \\
0.00 \\
0.51 \\
1.37 \\
0.00 \\
0.30\end{array}$ & $\begin{array}{l}\text { Un } \\
29.4 \\
0.00 \\
6.76 \\
0.00 \\
0.56 \\
1.33 \\
0.04 \\
0.26\end{array}$ & $\begin{array}{c}\text { Cel1 Cc } \\
29.8 \\
0.00 \\
6.20 \\
0.00 \\
0.25 \\
1.12 \\
0.00 \\
0.45\end{array}$ & $\begin{array}{c}\text { sition } \\
29.4 \\
0.00 \\
6.75 \\
0.00 \\
0.54 \\
1.22 \\
0.00 \\
0.37\end{array}$ & $\begin{array}{c}\text { ased on } \\
29.3 \\
0.00 \\
6.78 \\
0.00 \\
0.76 \\
1.15 \\
0.00 \\
0.31\end{array}$ & $\begin{array}{c}(0) \\
29.3 \\
0.00 \\
6.75 \\
0.00 \\
0.52 \\
1.37 \\
0.00 \\
0 .{ }^{\circ} 38\end{array}$ & $\begin{array}{c}29.3 \\
0.00 \\
6.82 \\
0.00 \\
0.47 \\
1.33 \\
0.00 \\
0.33\end{array}$ & $\begin{array}{c}29.4 \\
0.00 \\
6.63 \\
0.00 \\
0.34 \\
1.15 \\
0.00 \\
0.32\end{array}$ \\
\hline K & 2.24 & 2.17 & 2.98 & 2.37 & 2.18 & 2.32 & 2.26 & 3.33 \\
\hline \multicolumn{8}{|c|}{$\mathrm{Si} /(\mathrm{Al}+\mathrm{Fe})$} & 4.43 \\
\hline \multicolumn{9}{|c|}{$(\mathrm{Al}+\mathrm{Fe}) /(2 \mathrm{Mg}+2 \mathrm{Ca}+2 \mathrm{Ba}+\mathrm{Na}+\mathrm{K})$} \\
\hline $\begin{array}{l}\mathrm{K} \\
\mathrm{Na} \\
\mathrm{Ca}+\mathrm{Mg}\end{array}$ & $\begin{array}{r}50.6 \\
6.9 \\
42.5\end{array}$ & $\begin{array}{r}50.2 \\
6.0 \\
43.8\end{array}$ & $\begin{array}{c}\text { Mol\% E } \\
62.0 \\
9.3 \\
28.7\end{array}$ & $\begin{array}{c}\text { angeabl } \\
52.5 \\
8.3 \\
39.2\end{array}$ & $\begin{array}{c}\text { Cations } \\
49.5 \\
7.0 \\
43.5\end{array}$ & $\begin{array}{r}50.5 \\
8.3 \\
41.2\end{array}$ & $\begin{array}{r}51.5 \\
7.5 \\
41.0\end{array}$ & $\begin{array}{r}64.8 \\
6.2 \\
29.0\end{array}$ \\
\hline
\end{tabular}




\section{APPENDIX $F($ cont $)$}

Outcrop at Prow Pass, Northern Yucca Mountain

Sample Number: 3-15-82-6; Nevada State Coordinates (ft) 782850N, 550625E Nonwelded zeolitic tuff in central part of tuff of Calico Hills

\begin{tabular}{|c|c|c|c|c|c|c|c|c|}
\hline $\begin{array}{l}\mathrm{Si} 02 \\
\mathrm{TiO} 02 \\
\mathrm{Al} 203 \\
\mathrm{Fe} 203 \\
\mathrm{MgO} \\
\mathrm{CaO} \\
\mathrm{BaO} \\
\mathrm{Na} 20 \\
\mathrm{~K} 20\end{array}$ & $\begin{array}{c}61.0 \\
0.00 \\
12.4 \\
0.54 \\
0.45 \\
2.27 \\
0.00 \\
0.18 \\
5.61\end{array}$ & $\begin{array}{c}65.8 \\
0.00 \\
12.4 \\
0.00 \\
0.50 \\
2.36 \\
0.00 \\
0.29 \\
5.69\end{array}$ & $\begin{array}{c}64.2 \\
0.00 \\
12.0 \\
0.00 \\
0.51 \\
2.20 \\
0.00 \\
0.30 \\
6.03\end{array}$ & $\begin{array}{c}61.0 \\
0.00 \\
12.6 \\
0.18 \\
0.46 \\
2.25 \\
0.00 \\
0.32 \\
4.86\end{array}$ & $\begin{array}{c}66.8 \\
0.00 \\
12.8 \\
0.00 \\
0.52 \\
2.41 \\
0.00 \\
0.26 \\
4.83\end{array}$ & $\begin{array}{c}65.5 \\
0.00 \\
12.7 \\
0.00 \\
0.43 \\
2.38 \\
0.00 \\
0.39 \\
6.12\end{array}$ & $\begin{array}{c}66.7 \\
0.00 \\
11.7 \\
0.00 \\
0.38 \\
2.08 \\
0.00 \\
0.26 \\
4.76\end{array}$ & $\begin{array}{c}63.3 \\
0.00 \\
11.9 \\
0.00 \\
0.42 \\
2.14 \\
0.00 \\
0.32 \\
6.09\end{array}$ \\
\hline Total & 82.5 & 87.1 & 85.3 & 81.6 & 87.6 & 87.5 & 85.9 & 84.2 \\
\hline $\begin{array}{l}\mathrm{Si} \\
\mathrm{Ti} \\
\mathrm{Al} \\
\mathrm{Fe} \\
\mathrm{Mg} \\
\mathrm{Ca} \\
\mathrm{Ba} \\
\mathrm{Na} \\
\mathrm{K}\end{array}$ & $\begin{array}{c}29.0 \\
0.00 \\
6.97 \\
0.19 \\
0.32 \\
1.16 \\
0.00 \\
0.17 \\
3.40\end{array}$ & $\begin{array}{c}\text { Unit } \\
29.5 \\
0.00 \\
6.57 \\
0.00 \\
0.33 \\
1.13 \\
0.00 \\
0.25 \\
3.25\end{array}$ & $\begin{array}{c}11 \text { Com } \\
29.5 \\
0.00 \\
6.50 \\
0.00 \\
0.35 \\
1.08 \\
0.00 \\
0.27 \\
3.53\end{array}$ & $\begin{array}{c}\text { sition } \\
29.1 \\
0.00 \\
7.07 \\
0.06 \\
0.33 \\
1.15 \\
0.00 \\
0.30 \\
2.96\end{array}$ & $\begin{array}{c}\text { sed on } \\
29.5 \\
0.00 \\
6.65 \\
0.00 \\
0.34 \\
1.14 \\
0.00 \\
0.22 \\
2.72\end{array}$ & $\begin{array}{l}(0) \\
29.3 \\
0.00 \\
6.69 \\
0.00 \\
0.29 \\
1.14 \\
0.00 \\
0.34 \\
3.49\end{array}$ & $\begin{array}{c}30.0 \\
0.00 \\
6.22 \\
0.00 \\
0.25 \\
1.00 \\
0.00 \\
0.23 \\
2.73\end{array}$ & $\begin{array}{c}29.5 \\
0.00 \\
6.52 \\
0.00 \\
0.29 \\
1.07 \\
0.00 \\
0.29 \\
3.61\end{array}$ \\
\hline \multicolumn{9}{|c|}{$\mathrm{Si} /(\mathrm{Al}+\mathrm{Fe})$} \\
\hline & 1.10 & $\begin{array}{r}1 \\
1.02\end{array}$ & $\begin{array}{r}+\mathrm{Fe}) /(2 \\
0.98\end{array}$ & $\begin{array}{r}+2 \mathrm{Ca}+2 \mathrm{~B} \\
1.15\end{array}$ & $\begin{array}{l}\mathrm{Na}+\mathrm{K}) \\
\quad 1.12\end{array}$ & 1.00 & 1.14 & 0.99 \\
\hline $\begin{array}{l}\mathrm{K} \\
\mathrm{Na} \\
\mathrm{Ca}+\mathrm{Mg}\end{array}$ & $\begin{array}{r}67.5 \\
3.3 \\
29.2\end{array}$ & $\begin{array}{c}{ }^{M} \\
65.4 \\
5.1 \\
29.5\end{array}$ & $\begin{array}{c}\text { Excha } \\
67.5 \\
5.1 \\
27.4\end{array}$ & $\begin{array}{r}\text { eable } \\
62.5 \\
6.3 \\
31.2\end{array}$ & $\begin{array}{r}\text { ions } \\
61.5 \\
5.0 \\
33.5\end{array}$ & $\begin{array}{r}66.4 \\
6.4 \\
27.1\end{array}$ & $\begin{array}{r}64.8 \\
5.4 \\
29.8\end{array}$ & $\begin{array}{r}68.7 \\
5.5 \\
25.8\end{array}$ \\
\hline
\end{tabular}




\section{APPENDIX F (cont)}

Compositions of Clinoptilolites in Outcrop at Prow Pass, Northern Yucca Mt. Sample Number: 3-15-82-2; Nevada State Coordinates (ft) 786600N, 550900E Nonwelded zeolitic tuff near base of tuff of Calico Hills

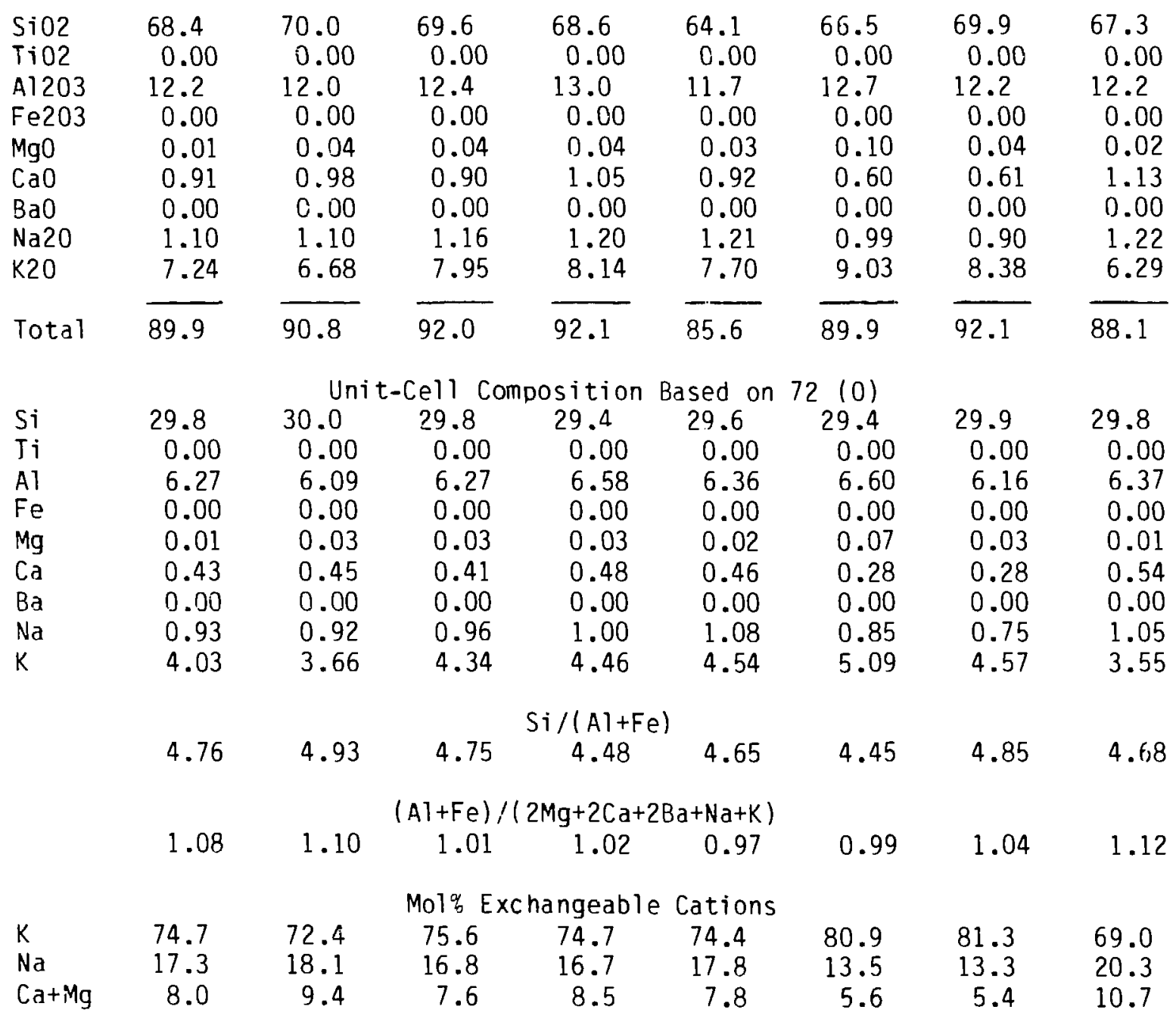


APPENDIX G

ANALCIME COMPOSITIONS DETERMINED BY ELECTRON MICROPROBE, YUCCA MOUNTAIN, NEVADA

Drill Hole USW G-1

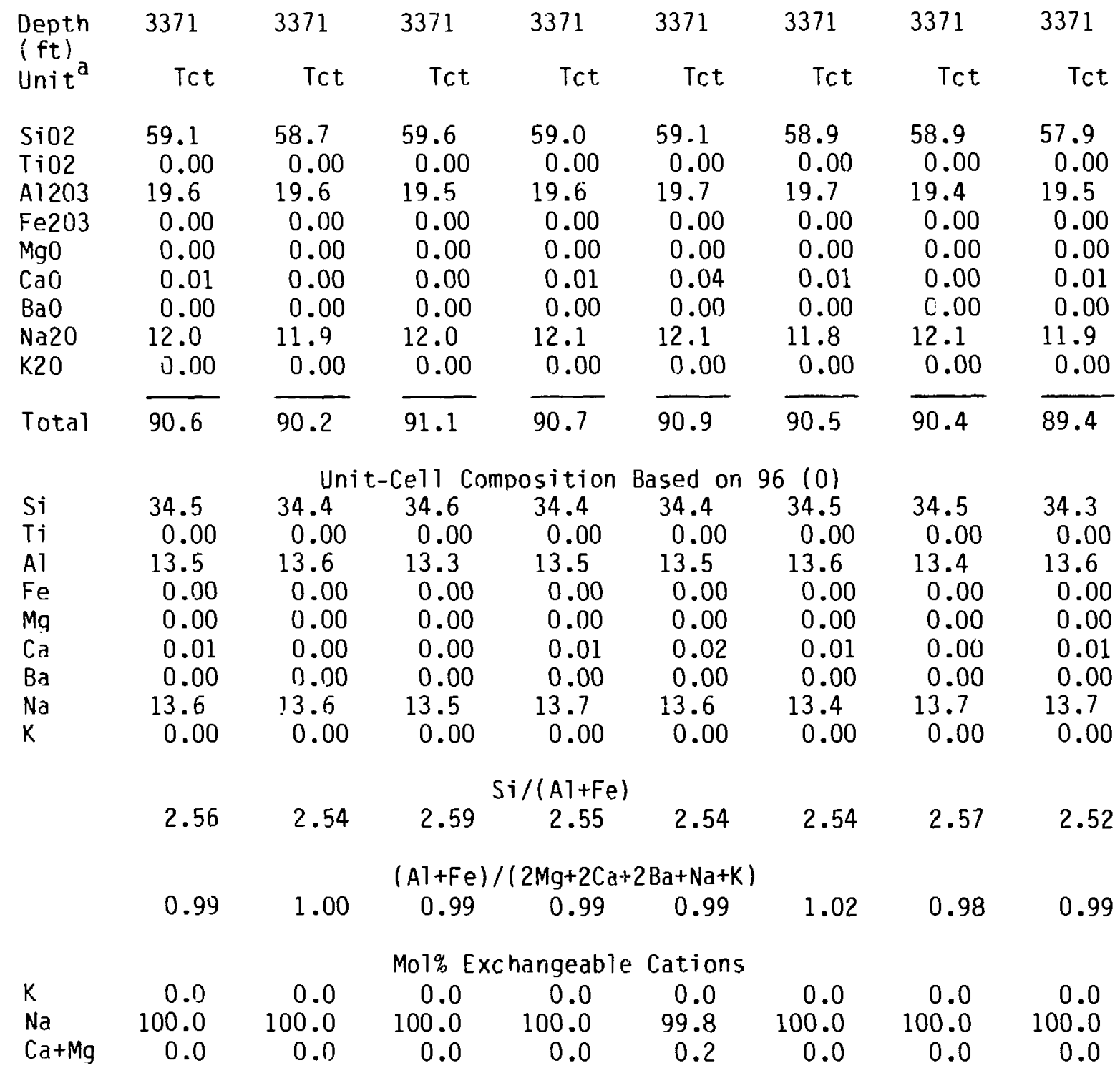

anit symbols defined in Table $I$. 


\section{APPENDIX G (cont.)}

Drill Hole USW G-1

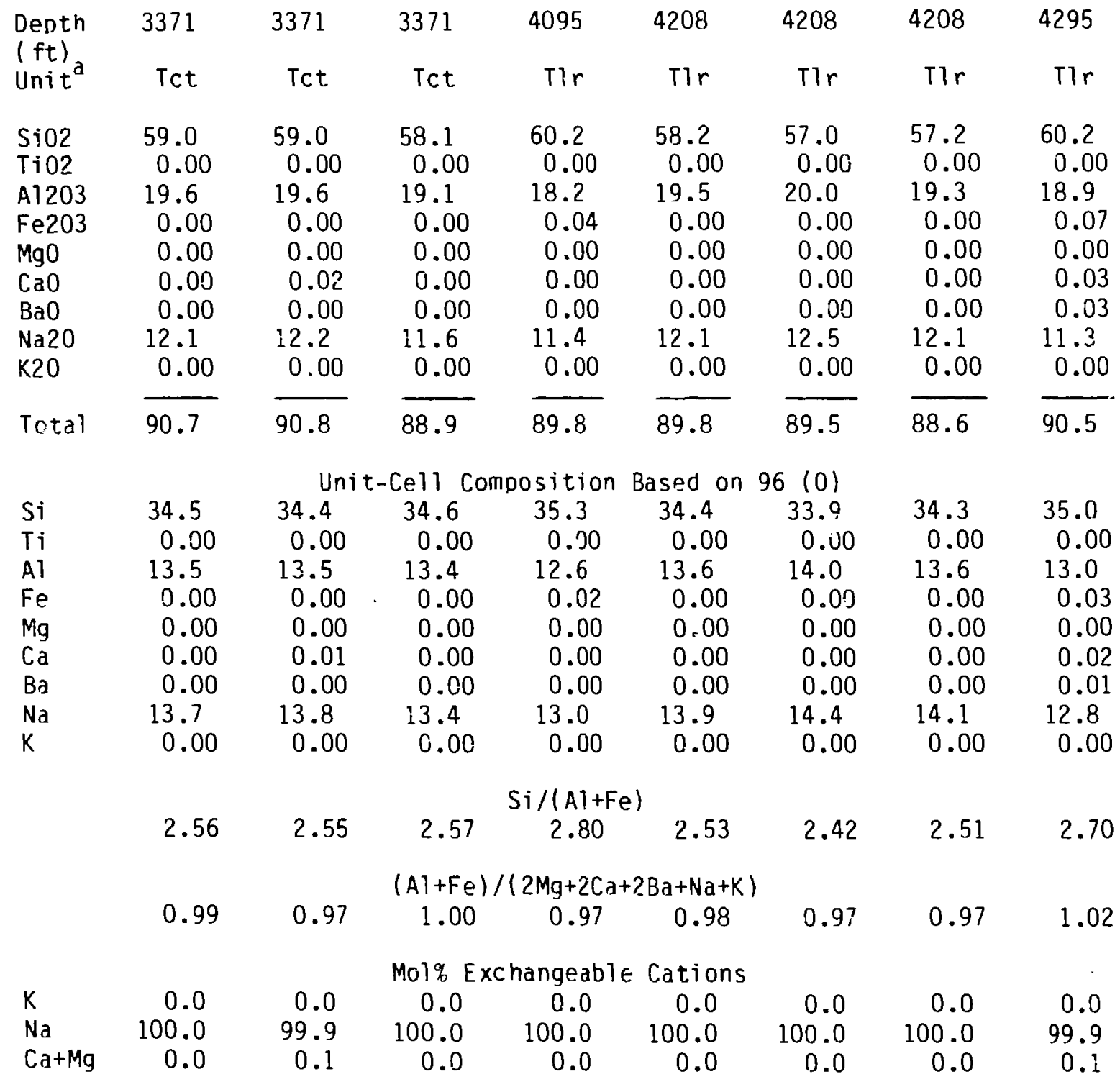




\section{APPENDIX G (cont)}

Drill Hole USW G-1

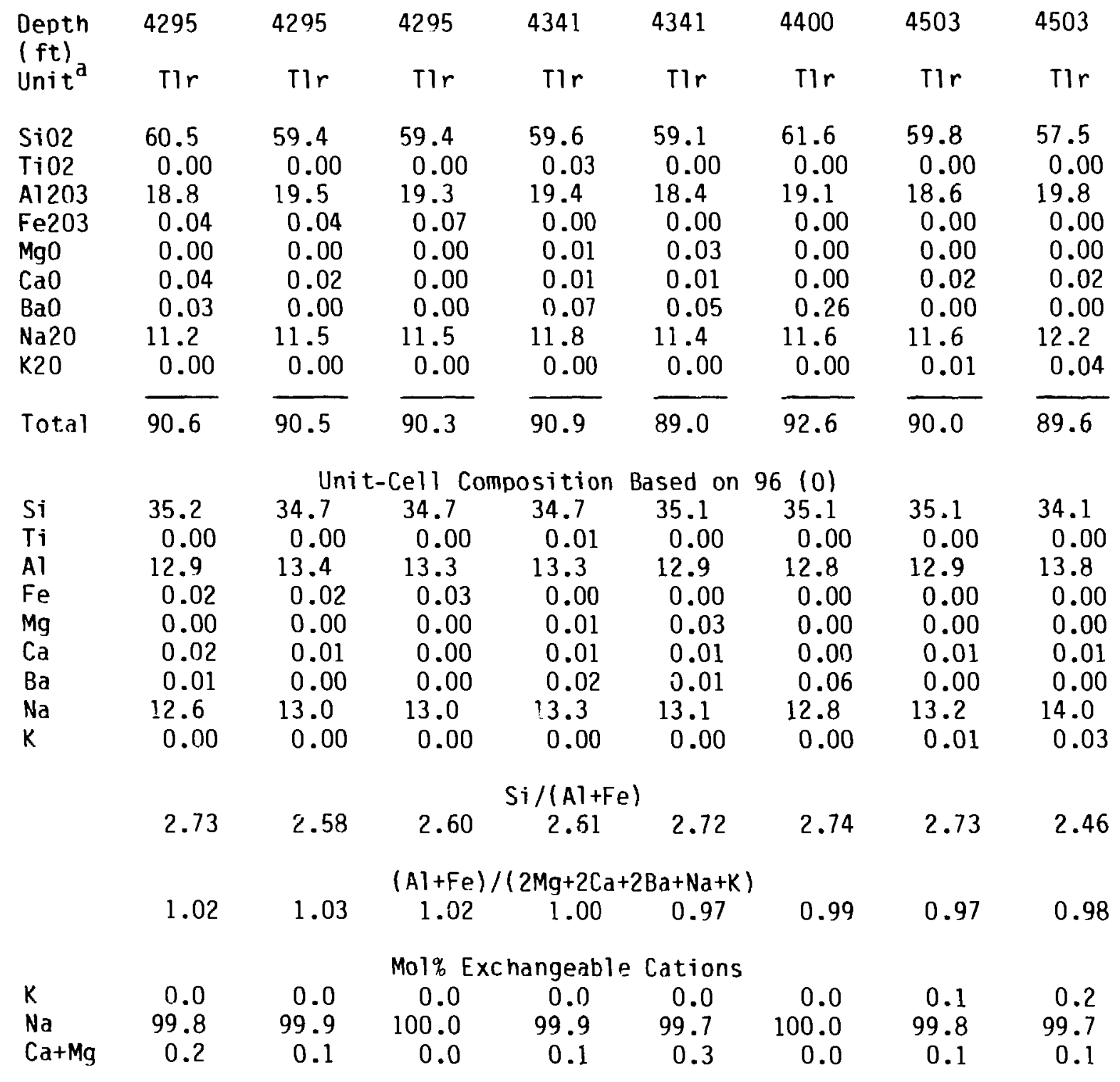




\section{APPENDIX G (cont)}

Drill Hole USW G-1

\begin{tabular}{|c|c|c|c|c|c|c|c|c|}
\hline \multirow{2}{*}{$\begin{array}{l}\text { Depth } \\
\text { (ft) } \\
\text { Unit }\end{array}$} & 4700 & 4700 & 47.00 & 4876 & 4876 & 4876 & 4876 & 4912 \\
\hline & TIr & $\operatorname{Tlr}$ & $\operatorname{Tl} r$ & Tlr & $\operatorname{Tl} r$ & $\operatorname{Tlr}$ & $T 1 r$ & $\operatorname{Tlr}$ \\
\hline $\begin{array}{l}\mathrm{Si} 02 \\
\mathrm{Ti} 02\end{array}$ & $\begin{array}{c}57.8 \\
0.00\end{array}$ & $\begin{array}{c}58.4 \\
0.00\end{array}$ & $\begin{array}{c}58.2 \\
0.00\end{array}$ & $\begin{array}{c}59.1 \\
0.05\end{array}$ & $\begin{array}{c}56.7 \\
0.00\end{array}$ & 59.9 & 57.3 & 57.1 \\
\hline TiO2 & 0.00 & 0.00 & 0.00 & 0.05 & 0.00 & 0.00 & 0.02 & 0.00 \\
\hline A1203 & 19.7 & 19.7 & 20.0 & 18.9 & 20.4 & 18.8 & 19.0 & 19.9 \\
\hline $\mathrm{Fe} 203$ & 0.04 & 0.01 & 0.04 & 0.00 & 0.00 & 0.01 & 0.00 & 0.01 \\
\hline $\mathrm{MgO}$ & 0.00 & 0.00 & 0.00 & 0.02 & 0.00 & 0.03 & 0.00 & 0.00 \\
\hline CaO & 0.00 & 0.00 & 0.00 & 0.00 & 0.00 & 0.02 & 0.00 & 0.00 \\
\hline $\mathrm{BaO}$ & 0.05 & 0.18 & 0.00 & 0.00 & 0.00 & 0.00 & 0.00 & 0.00 \\
\hline $\mathrm{Na} 20$ & 11.9 & 11.8 & 11.8 & 11.8 & 12.8 & 11.7 & 11.9 & 11.9 \\
\hline K20 & 0.00 & 0.00 & 0.00 & 0.00 & 0.02 & 0.03 & 0.03 & 0.00 \\
\hline \multirow[t]{2}{*}{ Total } & 89.5 & 90.1 & 90.0 & 89.9 & 89.9 & 90.5 & 88.3 & 88.9 \\
\hline & \multicolumn{8}{|c|}{ Unit-Cell Composition Based on $96(0)$} \\
\hline $\mathrm{Si}$ & 34.2 & 34.4 & 34.2 & 34.8 & 33.6 & 35.0 & 34.4 & 34.1 \\
\hline $\mathrm{Ti}$ & 0.00 & 0.00 & 0.00 & 0.02 & 0.00 & 0.00 & 0.01 & 0.00 \\
\hline Al & 13.8 & 13.7 & 13.9 & 13.1 & 14.3 & 12.9 & 13.5 & 14.0 \\
\hline $\mathrm{Fe}$ & 0.02 & 0.00 & 0.02 & 0.00 & 0.00 & 0.00 & 0.00 & 0.00 \\
\hline $\mathrm{Mg}$ & 0.00 & 0.00 & 0.00 & 0.02 & 0.00 & 0.03 & 0.00 & 0.00 \\
\hline $\mathrm{Ca}$ & 0.00 & 0.00 & 0.00 & 0.00 & 0.00 & 0.01 & 0.00 & 0.00 \\
\hline $\mathrm{Ba}$ & 0.01 & 0.04 & 0.00 & 0.00 & 0.00 & 0.00 & 0.00 & 0.00 \\
\hline $\mathrm{Na}$ & 13.7 & 13.5 & 13.5 & 13.5 & 14.7 & 13.2 & 13.9 & 13.8 \\
\hline \multirow[t]{6}{*}{$k$} & 0.00 & 0.00 & 0.00 & 0.00 & 0.02 & 0.02 & 0.02 & 0.00 \\
\hline & \multicolumn{8}{|c|}{$\mathrm{Si} /(\mathrm{Al}+\mathrm{Fe})$} \\
\hline & 2.49 & 2.51 & 2.47 & 2.65 & 2.36 & 2.70 & 2.56 & 2.43 \\
\hline & \multicolumn{8}{|c|}{$(\mathrm{Al}+\mathrm{Fe}) /(2 \mathrm{Mg}+2 \mathrm{Ca}+2 \mathrm{Ba}+\mathrm{Na}+\mathrm{K})$} \\
\hline & 1.01 & 1.01 & 1.03 & 0.97 & 0.97 & 0.97 & 0.97 & 1.02 \\
\hline & \multicolumn{8}{|c|}{ Mol\% Exchangeable Cations } \\
\hline $\mathrm{k}$ & 0.0 & 0.0 & 0.0 & 0.0 & 0.1 & 0.2 & 0.2 & 0.0 \\
\hline $\mathrm{Na}$ & 100.0 & 100.0 & 100.0 & 99.9 & 99.9 & 99.5 & 99.8 & 100.0 \\
\hline $\mathrm{Ca}+\mathrm{Mg}$ & 0.0 & 0.0 & 0.0 & 0.1 & 0.0 & 0.3 & 0.0 & 0.0 \\
\hline
\end{tabular}


APPENDIX G (cont)

Drill Hole USW G-1

\begin{tabular}{|c|c|c|c|c|c|c|c|c|}
\hline Depth & 4912 & 4912 & 5093 & 5126 & 5126 & 5167 & 5167 & 5167 \\
\hline Unit ${ }^{a}$ & $T 1 r$ & Tlr & Tot & Tot & Tot & Tot & Tot & Tot \\
\hline $\begin{array}{l}\mathrm{SiO} 02 \\
\mathrm{Ti02} \\
\mathrm{Al} 203 \\
\mathrm{Fe} 203 \\
\mathrm{MgO} \\
\mathrm{CaO} \\
\mathrm{BaO} \\
\mathrm{Na2O} \\
\mathrm{K} 2 \mathrm{O}\end{array}$ & $\begin{array}{c}58.4 \\
0.00 \\
20.0 \\
0.04 \\
0.00 \\
0.00 \\
0.00 \\
11.9 \\
0.01\end{array}$ & $\begin{array}{c}57.0 \\
0.00 \\
20.8 \\
0.00 \\
0.00 \\
0.00 \\
0.00 \\
12.5 \\
0.00\end{array}$ & $\begin{array}{c}59.4 \\
0.00 \\
20.0 \\
0.00 \\
0.00 \\
0.00 \\
0.00 \\
11.8 \\
0.00\end{array}$ & $\begin{array}{c}58.6 \\
0.00 \\
19.5 \\
0.01 \\
0.01 \\
0.03 \\
0.00 \\
12.2 \\
0.01\end{array}$ & $\begin{array}{c}59.3 \\
0.00 \\
19.9 \\
0.03 \\
0.00 \\
0.00 \\
0.03 \\
12.5 \\
0.02\end{array}$ & $\begin{array}{c}57.5 \\
0.00 \\
19.9 \\
0.01 \\
0.00 \\
0.00 \\
0.05 \\
12.0 \\
0.00\end{array}$ & $\begin{array}{c}57.4 \\
0.00 \\
19.7 \\
0.00 \\
0.00 \\
0.01 \\
0.11 \\
11.6 \\
0.00\end{array}$ & $\begin{array}{c}58.4 \\
0.00 \\
18.0 \\
0.01 \\
0.00 \\
0.00 \\
0.00 \\
11.2 \\
0.00\end{array}$ \\
\hline Total & 90.4 & 90.3 & 91.2 & 90.4 & 91.8 & 89.5 & 88.8 & 87.6 \\
\hline $\begin{array}{l}\mathrm{Si} \\
\mathrm{Ti} \\
\mathrm{Al} \\
\mathrm{Fe} \\
\mathrm{Mg} \\
\mathrm{Ca} \\
\mathrm{Ba} \\
\mathrm{Na} \\
\mathrm{K}\end{array}$ & $\begin{array}{c}34.2 \\
0.00 \\
13.8 \\
0.02 \\
0.00 \\
0.00 \\
0.00 \\
13.5 \\
0.01\end{array}$ & $\begin{array}{c}\text { Uni } \\
33.6 \\
0.00 \\
14.5 \\
0.00 \\
0.00 \\
0.00 \\
0.00 \\
14.3 \\
0.00\end{array}$ & $\begin{array}{c}\text { Cel1 Co } \\
34.4 \\
0.00 \\
13.7 \\
0.00 \\
0.00 \\
0.00 \\
0.00 \\
13.3 \\
0.00\end{array}$ & $\begin{array}{c}\text { osition } \\
34.4 \\
0.00 \\
13.5 \\
0.00 \\
0.01 \\
0.02 \\
0.00 \\
13.9 \\
0.01\end{array}$ & $\begin{array}{c}\text { Based on } \\
34.3 \\
0.00 \\
13.6 \\
0.01 \\
0.00 \\
0.00 \\
0.01 \\
14.0 \\
0.01\end{array}$ & $\begin{array}{c}96(0) \\
34.1 \\
0.00 \\
13.9 \\
0.00 \\
0.00 \\
0.00 \\
0.01 \\
13.8 \\
0.00\end{array}$ & $\begin{array}{c}34.2 \\
0.00 \\
13.9 \\
0.00 \\
0.00 \\
0.01 \\
0.03 \\
13.4 \\
0.00\end{array}$ & $\begin{array}{c}35.1 \\
0.00 \\
12.8 \\
0.00 \\
0.00 \\
0.00 \\
0.00 \\
13.1 \\
0.00\end{array}$ \\
\hline & 2.47 & 2.32 & 2.52 & $\begin{array}{c}\mathrm{Si} /(\mathrm{Al}+\mathrm{F} \\
2.55\end{array}$ & 2.53 & 2.45 & 2.47 & 2.75 \\
\hline & 1.02 & 1.01 & $\begin{array}{c}(A 1+F e) \\
1.03\end{array}$ & $\begin{array}{c}2 \mathrm{Mg}+2 \mathrm{Ca} \\
0.97\end{array}$ & $\begin{array}{c}2 \mathrm{Ba}+\mathrm{Na}+\mathrm{K}) \\
0.97\end{array}$ & 1.01 & 1.03 & 0.98 \\
\hline $\begin{array}{l}\mathrm{K} \\
\mathrm{Na} \\
\mathrm{Ca}+\mathrm{Mg}\end{array}$ & $\begin{array}{c}0.1 \\
99.9 \\
0.0\end{array}$ & $\begin{array}{c}0.0 \\
100.0 \\
0.0\end{array}$ & $\begin{array}{c}\text { Mol\% Ex } \\
0.0 \\
100.0 \\
0.0\end{array}$ & $\begin{array}{c}\text { langeabl } \\
0.1 \\
99.7 \\
0.2\end{array}$ & $\begin{array}{c}\text { Cations } \\
0.1 \\
99.9 \\
0.0\end{array}$ & $\begin{array}{c}0.0 \\
100.0 \\
0.0\end{array}$ & $\begin{array}{c}0.0 \\
100.0 \\
0.0\end{array}$ & $\begin{array}{r}0.0 \\
100.0 \\
0.0\end{array}$ \\
\hline
\end{tabular}


APPENDIX G (cont)

Drill Hole USW G-1

\begin{tabular}{|c|c|c|c|c|c|c|c|c|}
\hline $\begin{array}{l}\text { Depth } \\
\text { ( ft) } \\
\text { Unit }^{\mathrm{a}}\end{array}$ & 5167 & 5212 & 5212 & 5212 & 5212 & 5296 & 5296 & 5296 \\
\hline $\begin{array}{l}\mathrm{Si02} \\
\mathrm{Ti02} \\
\mathrm{A} 1203 \\
\mathrm{Fe} 203 \\
\mathrm{Mg0} \\
\mathrm{Ca0} \\
\mathrm{BaO} \\
\mathrm{Na20} \\
\mathrm{K} 20\end{array}$ & $\begin{array}{c}57.8 \\
0.00 \\
20.1 \\
0.00 \\
0.00 \\
0.04 \\
0.00 \\
12.6 \\
0.00\end{array}$ & $\begin{array}{c}60.4 \\
0.00 \\
18.6 \\
0.05 \\
0.00 \\
0.01 \\
0.08 \\
11.3 \\
0.03\end{array}$ & $\begin{array}{c}59.4 \\
0.00 \\
18.5 \\
0.00 \\
0.01 \\
0.02 \\
0.13 \\
11.3 \\
0.03\end{array}$ & $\begin{array}{c}58.7 \\
0.00 \\
19.4 \\
0.10 \\
0.02 \\
0.02 \\
0.05 \\
11.7 \\
0.00\end{array}$ & $\begin{array}{c}59.8 \\
0.00 \\
18.9 \\
0.04 \\
0.00 \\
0.02 \\
0.05 \\
11.7 \\
0.02\end{array}$ & $\begin{array}{c}56.5 \\
0.00 \\
19.5 \\
0.02 \\
0.00 \\
0.00 \\
0.12 \\
11.8 \\
0.00\end{array}$ & $\begin{array}{c}59.1 \\
0.00 \\
20.0 \\
0.04 \\
0.00 \\
0.00 \\
0.09 \\
11.9 \\
0.00\end{array}$ & $\begin{array}{c}59.0 \\
0.00 \\
19.8 \\
0.04 \\
0.00 \\
0.00 \\
0.00 \\
12.0 \\
0.00\end{array}$ \\
\hline Total & 90.5 & 90.5 & 89.4 & 90.0 & 90.5 & 87.9 & 91.1 & 90.8 \\
\hline $\begin{array}{l}\mathrm{Si} \\
\mathrm{Ti} \\
\mathrm{Al} \\
\mathrm{Fe} \\
\mathrm{Mg} \\
\mathrm{Ca} \\
\mathrm{Ba} \\
\mathrm{Na} \\
\mathrm{K}\end{array}$ & $\begin{array}{c}34.0 \\
0.00 \\
13.9 \\
0.00 \\
0.00 \\
0.03 \\
0.00 \\
14.4 \\
0.00\end{array}$ & $\begin{array}{c}\text { Uni } \\
35.2 \\
0.00 \\
12.8 \\
0.02 \\
0.00 \\
0.01 \\
0.02 \\
12.8 \\
0.02\end{array}$ & $\begin{array}{c}\mathrm{Ce} 11 \mathrm{Co} \\
35.1 \\
0.00 \\
12.9 \\
0.00 \\
0.01 \\
0.01 \\
0.03 \\
12.9 \\
0.02\end{array}$ & $\begin{array}{c}\text { position } \\
34.5 \\
0.00 \\
13.5 \\
0.04 \\
0.02 \\
0.01 \\
0.01 \\
13.3 \\
0.00\end{array}$ & $\begin{array}{c}\text { Based on } \\
34.9 \\
0.00 \\
13.0 \\
0.02 \\
0.00 \\
0.01 \\
0.01 \\
13.2 \\
0.01\end{array}$ & $\begin{array}{c}96(0) \\
34.1 \\
0.00 \\
13.9 \\
0.01 \\
0.00 \\
0.00 \\
0.03 \\
13.8 \\
0.00\end{array}$ & $\begin{array}{c}34.3 \\
0.00 \\
13.7 \\
0.02 \\
0.00 \\
0.00 \\
0.02 \\
13.4 \\
0.00\end{array}$ & $\begin{array}{c}34.4 \\
0.00 \\
13.6 \\
0.02 \\
0.00 \\
0.00 \\
0.00 \\
13.6 \\
0.00\end{array}$ \\
\hline & 2.44 & 2.75 & $\begin{array}{c}2.72 \\
(\mathrm{~A} 1+\mathrm{Fe}) \\
0.99\end{array}$ & $\begin{array}{c}\mathrm{Si} /(\mathrm{A})+\mathrm{F} \\
2.56\end{array}$ & $\begin{array}{c}2 \mathrm{Ba}+\mathrm{Na} a+\mathrm{K} \\
0.98\end{array}$ & 2.46 & 2.50 & 1.00 \\
\hline $\begin{array}{l}\mathrm{K} \\
\mathrm{Na} \\
\mathrm{Ca}+\mathrm{Mg}\end{array}$ & $\begin{array}{r}0.0 \\
99.8 \\
0.2\end{array}$ & $\begin{array}{r}0.2 \\
99.8 \\
0.0\end{array}$ & $\begin{array}{c}\text { Mol\% E } \\
0.2 \\
99.7 \\
0.2\end{array}$ & $\begin{array}{c}\text { nangeabl } \\
0.0 \\
99.8 \\
0.2\end{array}$ & $\begin{array}{c}\text { Cations } \\
0.1 \\
99.8 \\
0.1\end{array}$ & $\begin{array}{r}0.0 \\
100.0 \\
0.0\end{array}$ & $\begin{array}{r}0.0 \\
100.0 \\
0.0\end{array}$ & $\begin{array}{r}0.0 \\
100.0 \\
0.0\end{array}$ \\
\hline
\end{tabular}


APPENDIX G (cont)

Drill Hole USW G-1

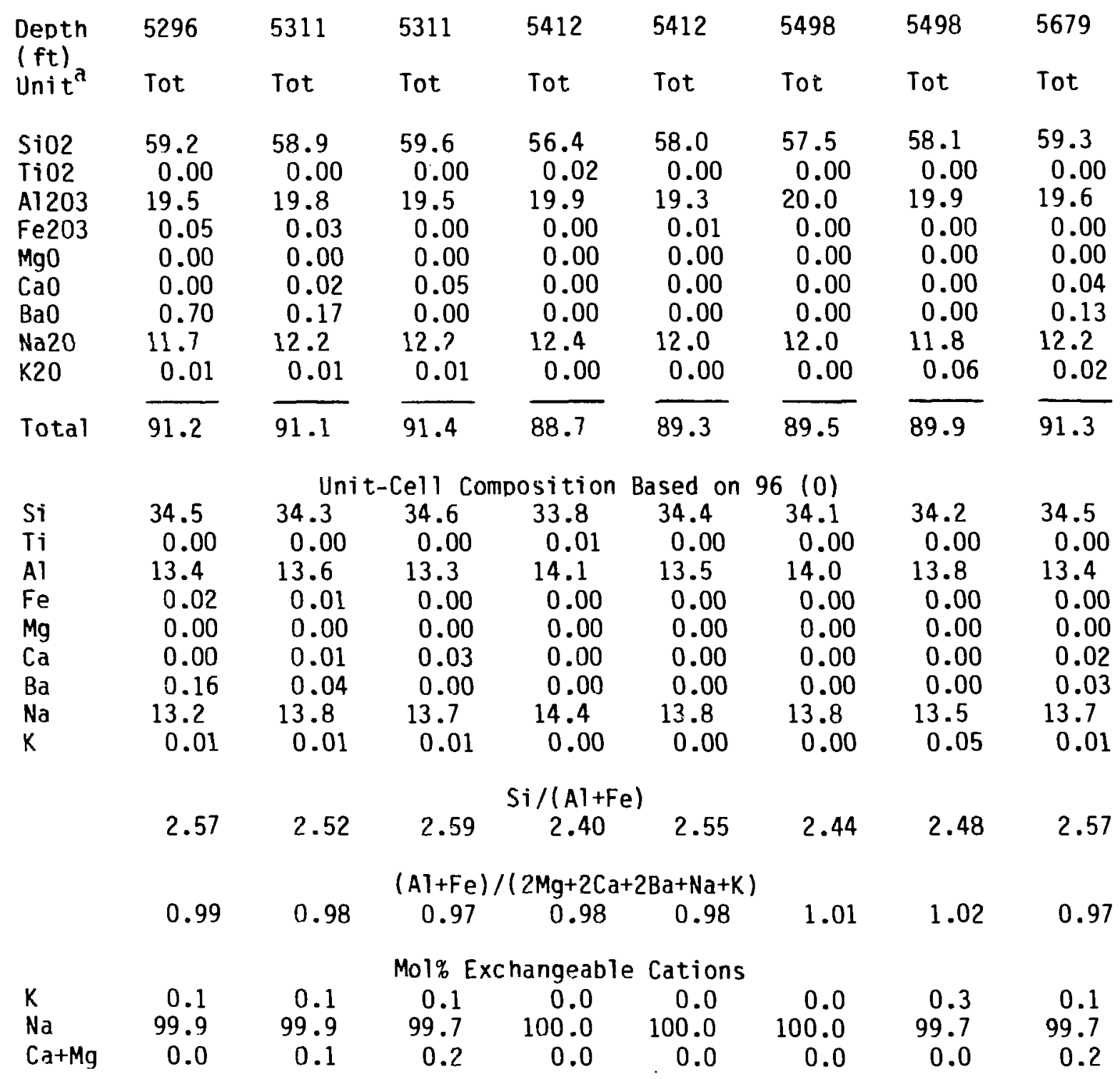




\section{APPENDIX G (cont)}

Drill Hole USH G-1

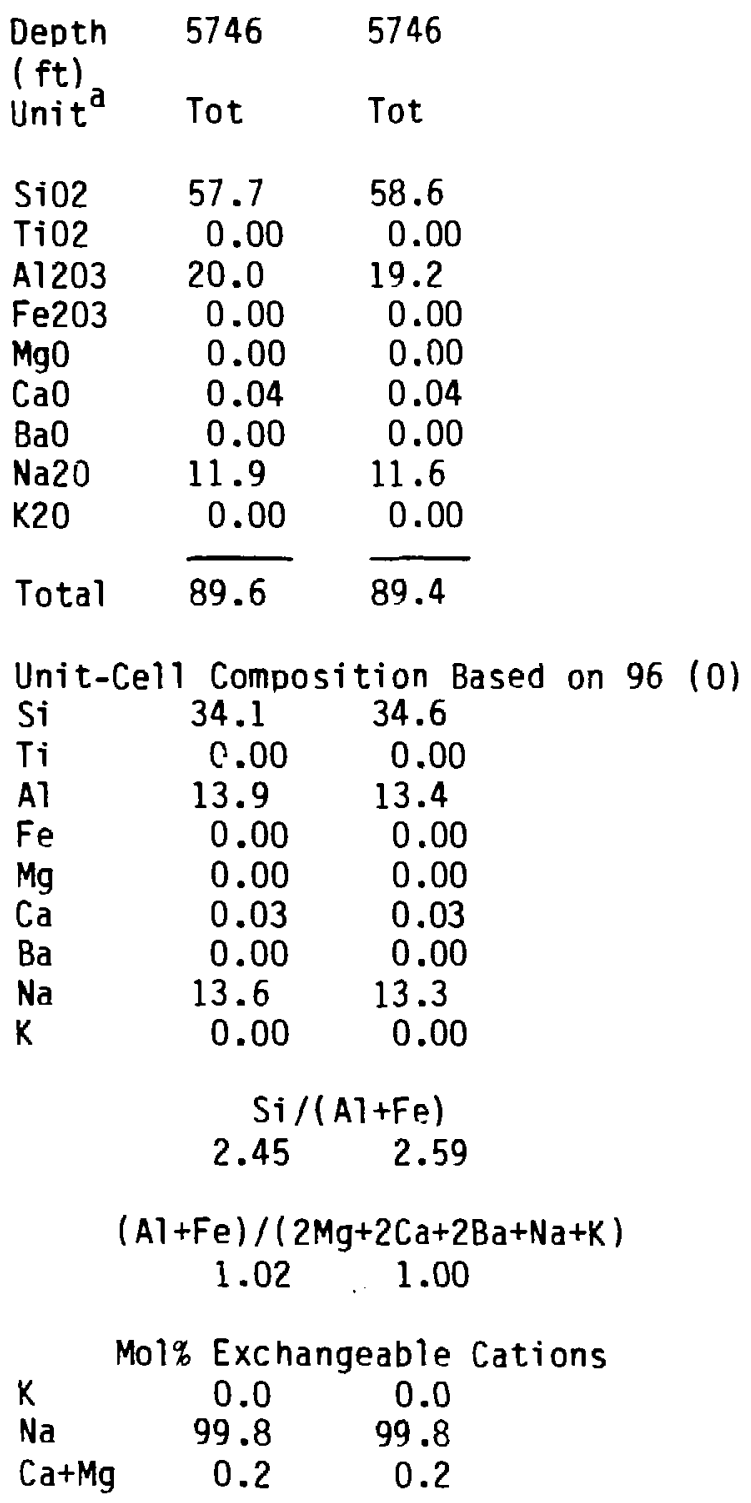


APPENDIX G (cont)

Drill Hole UE-25b\#1(H)

\begin{tabular}{|c|c|c|c|c|c|c|c|c|}
\hline \multirow{2}{*}{$\begin{array}{l}\text { Depth } \\
\text { (ft) } \\
\text { Unit }\end{array}$} & 3393 & 3393 & 3393 & 3393 & 3506 & 3506 & 3506 & 3506 \\
\hline & Tct & Tct & Tct & Tct & Tct & Tct & Tct & Tct \\
\hline $\begin{array}{l}\mathrm{SiO} 02 \\
\mathrm{Ti02} \\
\mathrm{Al} 203 \\
\mathrm{Fe} 203 \\
\mathrm{MgO} \\
\mathrm{CaO} \\
\mathrm{BaO} \\
\mathrm{Na} 20 \\
\mathrm{~K} 2 \mathrm{O}\end{array}$ & $\begin{array}{c}61.9 \\
0.00 \\
18.2 \\
0.00 \\
0.00 \\
0.00 \\
0.04 \\
11.3 \\
0.00\end{array}$ & $\begin{array}{c}61.3 \\
0.00 \\
18.5 \\
0.00 \\
0.00 \\
0.00 \\
0.00 \\
11.1 \\
0.00\end{array}$ & $\begin{array}{c}61.5 \\
0.00 \\
18.7 \\
0.00 \\
0.00 \\
0.02 \\
0.00 \\
11.3 \\
0.00\end{array}$ & $\begin{array}{c}60.7 \\
0.00 \\
18.6 \\
0.00 \\
0.00 \\
0.00 \\
0.01 \\
11.4 \\
0.00\end{array}$ & $\begin{array}{c}61.8 \\
0.00 \\
18.5 \\
0.00 \\
0.00 \\
0.00 \\
0.00 \\
11.2 \\
0.02\end{array}$ & $\begin{array}{c}61.6 \\
0.00 \\
19.0 \\
0.00 \\
0.00 \\
0.04 \\
0.06 \\
11.6 \\
0.00\end{array}$ & $\begin{array}{c}58.5 \\
0.00 \\
20.6 \\
0.00 \\
0.00 \\
0.03 \\
0.00 \\
12.7 \\
0.00\end{array}$ & $\begin{array}{c}57.6 \\
0.00 \\
20.8 \\
0.00 \\
0.00 \\
0.00 \\
0.01 \\
12.5 \\
0.01\end{array}$ \\
\hline Total & 91.3 & 91.0 & 91.5 & 90.8 & 91.5 & 92.4 & 91.8 & 90.9 \\
\hline $\begin{array}{l}\mathrm{Si} \\
\mathrm{Ti} \\
\mathrm{Al} \\
\mathrm{Fe} \\
\mathrm{Mg} \\
\mathrm{Ca} \\
\mathrm{Ba} \\
\mathrm{Na} \\
\mathrm{K}\end{array}$ & $\begin{array}{c}35.6 \\
0.00 \\
12.3 \\
0.00 \\
0.00 \\
0.00 \\
0.01 \\
12.6 \\
0.00\end{array}$ & $\begin{array}{c}\text { Uni } \\
35.4 \\
0.00 \\
12.6 \\
0.00 \\
0.00 \\
0.00 \\
0.00 \\
12.5 \\
0.00\end{array}$ & $\begin{array}{c}\text { Cel1 Co } \\
35.3 \\
0.00 \\
12.7 \\
0.00 \\
0.00 \\
0.01 \\
0.00 \\
12.6 \\
0.00\end{array}$ & $\begin{array}{c}\text { osition } \\
35.2 \\
0.00 \\
12.8 \\
0.00 \\
0.00 \\
0.00 \\
0.00 \\
12.8 \\
0.00\end{array}$ & $\begin{array}{c}\text { Based on } \\
35.5 \\
0.00 \\
12.5 \\
0.00 \\
0.00 \\
0.00 \\
0.00 \\
12.5 \\
0.0 ?\end{array}$ & $\begin{array}{c}96 \quad(0) \\
35.2 \\
0.00 \\
12.8 \\
0.00 \\
0.00 \\
0.02 \\
0.01 \\
12.8 \\
0.00\end{array}$ & $\begin{array}{c}33.9 \\
0.00 \\
14.1 \\
0.00 \\
0.00 \\
0.02 \\
0.00 \\
14.3 \\
0.00\end{array}$ & $\begin{array}{c}33.7 \\
0.00 \\
14.3 \\
0.00 \\
0.00 \\
0.00 \\
0.00 \\
14.2 \\
0.01\end{array}$ \\
\hline \multirow{2}{*}{ K } & \multicolumn{8}{|c|}{$\mathrm{Si} /(\mathrm{Al}+\mathrm{Fe})$} \\
\hline & 0.98 & 1.01 & $\begin{array}{c}(\mathrm{A} 1+\mathrm{Fe}) \\
1.00\end{array}$ & $\begin{array}{c}2 \mathrm{Mg}+2 \mathrm{Ca}- \\
0.99\end{array}$ & $\begin{array}{c}2 \mathrm{Ba}+\mathrm{Na}+\mathrm{K}) \\
1.00\end{array}$ & 0.99 & 0.99 & 1.01 \\
\hline $\begin{array}{l}\mathrm{K} \\
\mathrm{Na} \\
\mathrm{Ca}+\mathrm{Mg}\end{array}$ & $\begin{array}{r}0.0 \\
100.0 \\
0.0\end{array}$ & $\begin{array}{r}0.0 \\
100.0 \\
0.0\end{array}$ & $\begin{array}{c}\text { Mol\% EX } \\
0.0 \\
99.9 \\
0.1\end{array}$ & $\begin{array}{c}\text { hangeab } 1 \\
0.0 \\
100.0 \\
0.0\end{array}$ & $\begin{array}{c}\text { Cations } \\
0.1 \\
99.9 \\
0.0\end{array}$ & $\begin{array}{r}0.0 \\
99.8 \\
0.2\end{array}$ & $\begin{array}{r}0.0 \\
99.9 \\
0.1\end{array}$ & $\begin{array}{r}0.1 \\
99.9 \\
0.0\end{array}$ \\
\hline
\end{tabular}


APPENDIX G (cont)

Drill Hole UE-25p\#1

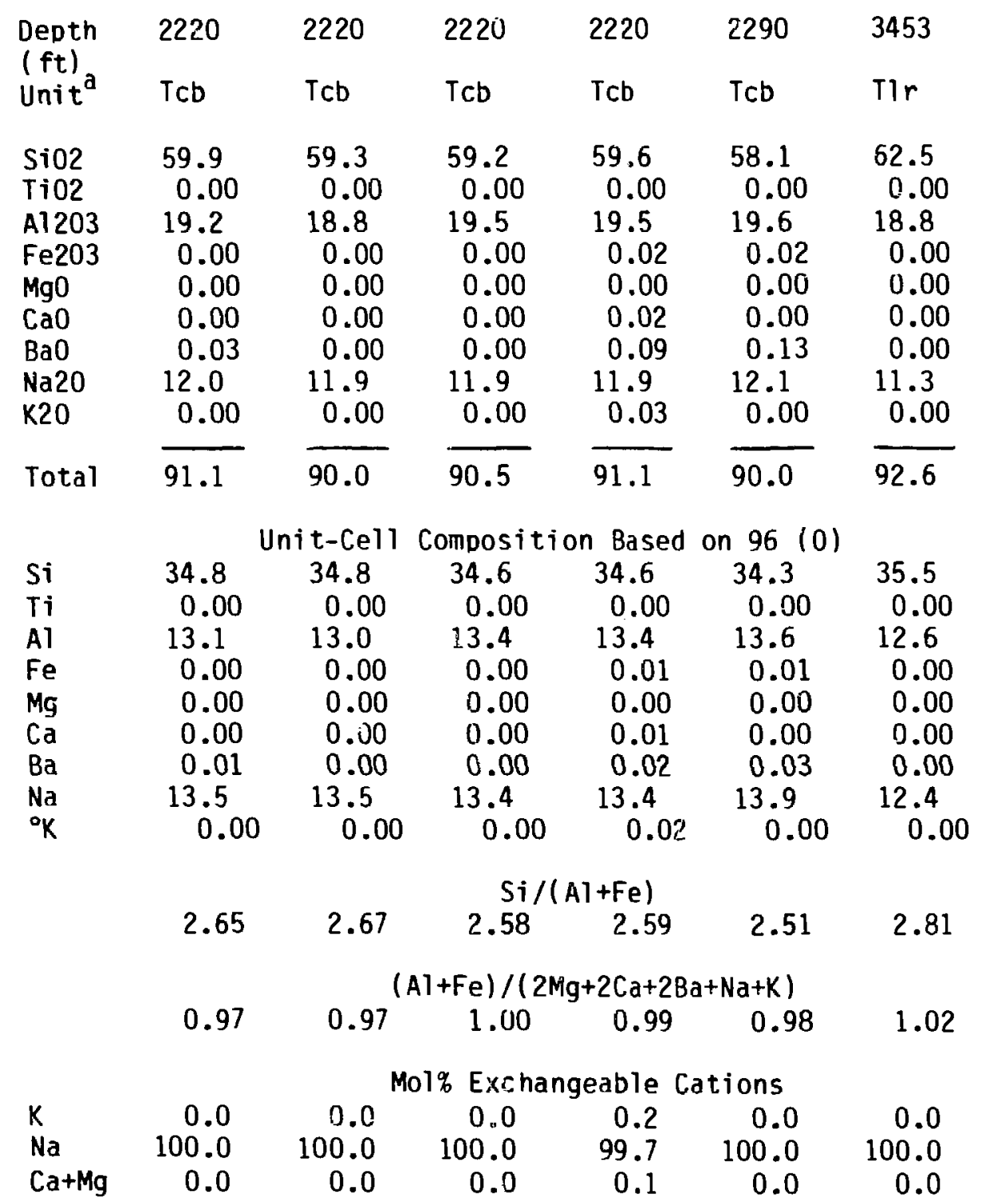


APPENDIX G (cont)

Drill Hole J-13

\begin{tabular}{|c|c|c|c|c|c|c|c|c|}
\hline $\begin{array}{l}\text { Depth } \\
\text { (ft) } \\
\text { Unit }\end{array}$ & 608 & 608 & 608 & 608 & 1995 & 1995 & 1995 & 1995 \\
\hline $\begin{array}{l}\mathrm{SiO} 02 \\
\mathrm{TiO} 02 \\
\mathrm{~A} 1203 \\
\mathrm{Fe} 203 \\
\mathrm{MgO} \\
\mathrm{CaO} \\
\mathrm{BaO} \\
\mathrm{Na} 20 \\
\mathrm{~K} 20\end{array}$ & $\begin{array}{c}58.6 \\
0.00 \\
19.3 \\
0.02 \\
0.00 \\
0.00 \\
0.16 \\
11.7 \\
0.03\end{array}$ & $\begin{array}{c}57.8 \\
0.00 \\
19.9 \\
0.04 \\
0.00 \\
0.00 \\
0.14 \\
12.0 \\
0.04\end{array}$ & $\begin{array}{c}57.5 \\
0.00 \\
19.3 \\
0.00 \\
0.00 \\
0.00 \\
0.00 \\
11.4 \\
0.00\end{array}$ & $\begin{array}{c}58.1 \\
0.00 \\
18.9 \\
0.07 \\
0.02 \\
0.00 \\
0.00 \\
11.7 \\
0.01\end{array}$ & $\begin{array}{c}60.1 \\
0.02 \\
19.3 \\
0.04 \\
0.01 \\
0.03 \\
0.13 \\
11.6 \\
0.03\end{array}$ & $\begin{array}{c}61.5 \\
0.00 \\
18.7 \\
0.05 \\
0.00 \\
0.00 \\
0.12 \\
11.2 \\
0.03\end{array}$ & $\begin{array}{c}59.2 \\
0.02 \\
20.2 \\
0.05 \\
0.00 \\
0.04 \\
0.05 \\
12.2 \\
0.05\end{array}$ & $\begin{array}{c}58.4 \\
0.00 \\
20.2 \\
0.00 \\
0.00 \\
0.00 \\
0.00 \\
12.1 \\
0.01\end{array}$ \\
\hline Total & 89.8 & 89.9 & 88.2 & 88.8 & 91.2 & 91.6 & 91.8 & 90.6 \\
\hline $\begin{array}{l}\mathrm{Si} \\
\mathrm{Ti} \\
\mathrm{Al} \\
\mathrm{Fe} \\
\mathrm{Mg} \\
\mathrm{Ca} \\
\mathrm{Ba} \\
\mathrm{Na} \\
\mathrm{K}\end{array}$ & $\begin{array}{c}34.6 \\
0.00 \\
13.4 \\
0.01 \\
0.00 \\
0.00 \\
0.04 \\
13.3 \\
0.02\end{array}$ & $\begin{array}{c}\text { Uni } \\
34.1 \\
0.00 \\
13.9 \\
0.02 \\
0.00 \\
0.00 \\
0.03 \\
13.7 \\
0.03\end{array}$ & $\begin{array}{c}\text { Cell cc } \\
34.5 \\
0.00 \\
13.6 \\
0.00 \\
0.60 \\
0.00 \\
0.00 \\
13.2 \\
0.00\end{array}$ & $\begin{array}{c}\text { position } \\
34.6 \\
0.00 \\
13.3 \\
0.03 \\
0.02 \\
0.00 \\
0.00 \\
13.6 \\
0.01\end{array}$ & $\begin{array}{c}\text { Based on } \\
34.8 \\
0.01 \\
13.2 \\
0.02 \\
0.01 \\
0.02 \\
0.03 \\
13.0 \\
0.02\end{array}$ & $\begin{array}{c}96(0) \\
35.3 \\
0.00 \\
12.7 \\
0.02 \\
0.00 \\
0.00 \\
0.03 \\
12.5 \\
0.02\end{array}$ & $\begin{array}{c}34.2 \\
0.01 \\
13.8 \\
0.02 \\
0.00 \\
0.02 \\
0.01 \\
13.7 \\
0.04\end{array}$ & $\begin{array}{c}34.1 \\
0.00 \\
13.9 \\
0.00 \\
0.00 \\
0.00 \\
0.00 \\
13.7 \\
0.01\end{array}$ \\
\hline \multicolumn{9}{|c|}{$\begin{array}{c}\mathrm{Si} /(\mathrm{Al}+\mathrm{Fe}) \\
2.60\end{array}$} \\
\hline & 1.00 & 1.01 & $(A]+F e) /(2 M g+2 C a+2 B a+N a+K)$ & $\begin{array}{r}(2 \mathrm{Mg}+2 \mathrm{C} \\
0.98\end{array}$ & $\begin{array}{c}-2 \mathrm{Ba}+\mathrm{Na}+\mathrm{K} \\
1.00\end{array}$ & 1.01 & 1.00 & 1.01 \\
\hline $\begin{array}{l}\mathrm{K} \\
\mathrm{Na} \\
\mathrm{Ca}+\mathrm{Mg}\end{array}$ & $\begin{array}{r}0.2 \\
99.8 \\
0.0\end{array}$ & $\begin{array}{r}0.2 \\
99.8 \\
0.0\end{array}$ & $\begin{array}{c}\text { Mol\% E } \\
0.0 \\
100.0 \\
0.0\end{array}$ & $\begin{array}{c}\text { hangeab } \\
0.1 \\
99.8 \\
0.1\end{array}$ & $\begin{array}{c}\text { Cations } \\
0.2 \\
99.6 \\
0.2\end{array}$ & $\begin{array}{r}0.2 \\
99.8 \\
0.0\end{array}$ & $\begin{array}{r}0.3 \\
99.6 \\
0.2\end{array}$ & $\begin{array}{r}0.1 \\
99.9 \\
0.0\end{array}$ \\
\hline
\end{tabular}




\begin{tabular}{|c|c|c|}
\hline \multicolumn{3}{|c|}{ APPENDIX G (cont) } \\
\hline $\begin{array}{l}\text { Depth } \\
(\mathrm{ft}) \\
\text { Unit }^{\mathrm{a}}\end{array}$ & $\begin{array}{l}1995 \\
\text { TCD }\end{array}$ & $\begin{array}{l}1995 \\
\text { TCD }\end{array}$ \\
\hline $\begin{array}{l}\mathrm{SiO} 2 \\
\mathrm{TiO} 02 \\
\mathrm{~A} 1203 \\
\mathrm{Fe} 203 \\
\mathrm{MgO} \\
\mathrm{CaO} \\
\mathrm{BaO} \\
\mathrm{Na} 20 \\
\mathrm{~K} 2 \mathrm{O}\end{array}$ & $\begin{array}{c}51.4 \\
0.00 \\
19.1 \\
0.02 \\
0.00 \\
0.02 \\
0.05 \\
11.2 \\
0.00\end{array}$ & $\begin{array}{c}60.3 \\
0.00 \\
18.9 \\
0.00 \\
0.00 \\
0.00 \\
0.09 \\
11.5 \\
0.01\end{array}$ \\
\hline Total & 91.9 & 90.9 \\
\hline $\begin{array}{l}\text { Unit-C } \\
96(0) \\
\mathrm{Si} \\
\mathrm{Ti} \\
\mathrm{AT} \\
\mathrm{Fe} \\
\mathrm{Mg} \\
\mathrm{Ca} \\
\mathrm{Ba} \\
\mathrm{Na} \\
\mathrm{K}\end{array}$ & $\begin{array}{c}\text { e11 Compo } \\
35.2 \\
0.00 \\
12.9 \\
0.01 \\
0.00 \\
0.01 \\
0.01 \\
12.5 \\
0.00\end{array}$ & 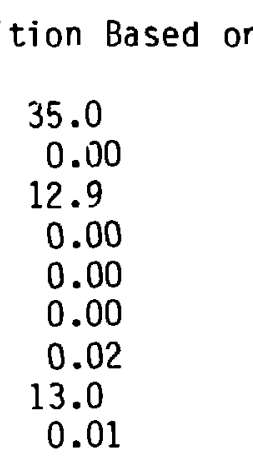 \\
\hline & $\begin{array}{c}\mathrm{Si} / / \mathrm{A} 1+ \\
2.72\end{array}$ & $2.7 i$ \\
\hline$(\mathrm{A})+\mathrm{Fe}$ & $\begin{array}{c}/(2 \mathrm{Mg}+2 \mathrm{C} \\
1.03\end{array}$ & $\begin{array}{c}+2 B a+N a+K) \\
1.00\end{array}$ \\
\hline $\begin{array}{l}\mathrm{Mol} \% \\
\mathrm{Ka} \\
\mathrm{Ca}+\mathrm{Mg}\end{array}$ & $\begin{array}{c}\text { Exchanged } \\
0.0 \\
99.9 \\
0.1\end{array}$ & $\begin{array}{c}\text { le Cations } \\
0.1 \\
99.9 \\
0.0\end{array}$ \\
\hline
\end{tabular}




\section{A.PPENDIX H}

AUTHIGENIC FELDSPAR COMPOSITIONS DETERMINED BY ELECTRON MICŔOPROBE, YUCCA MOUNTAIN, NEVADA

Drill Hole USW G-1

\begin{tabular}{|c|c|c|c|c|c|c|c|c|}
\hline Depth & 4208 & 4341 & 4400 & 4400 & 4503 & 4912 & 5026 & 5026 \\
\hline Unit ${ }^{a}$ & $\operatorname{Tlr}$ & $\operatorname{Tlr}$ & $T / r$ & $T / r$ & $\operatorname{Tlr}$ & $\operatorname{Tlr}$ & Tot & Tot \\
\hline $\begin{array}{l}\mathrm{SiO} 02 \\
\mathrm{Ti} 02 \\
\mathrm{~A} 1203 \\
\mathrm{Fe} 203 \\
\mathrm{MgO} \\
\mathrm{CaO} \\
\mathrm{BaO} \\
\mathrm{Na} 20 \\
\mathrm{~K} 20\end{array}$ & $\begin{array}{c}66.9 \\
0.00 \\
16.3 \\
0.00 \\
0.00 \\
0.00 \\
0.00 \\
0.04 \\
15.0\end{array}$ & $\begin{array}{c}67.8 \\
0.02 \\
19.6 \\
0.00 \\
0.01 \\
0.16 \\
0.00 \\
11.8 \\
0.01\end{array}$ & $\begin{array}{c}69.3 \\
0.00 \\
19.0 \\
0.00 \\
0.00 \\
0.00 \\
0.00 \\
11.1 \\
0.58\end{array}$ & $\begin{array}{c}69.4 \\
0.00 \\
19.8 \\
0.02 \\
0.00 \\
0.14 \\
0.13 \\
11.6 \\
0.05\end{array}$ & $\begin{array}{c}67.3 \\
0.02 \\
16.4 \\
0.01 \\
0.02 \\
0.04 \\
0.05 \\
0.07 \\
15.2\end{array}$ & $\begin{array}{c}67.0 \\
0.00 \\
16.6 \\
0.04 \\
0.00 \\
0.00 \\
0.16 \\
0.04 \\
14.9\end{array}$ & $\begin{array}{c}68.1 \\
0.00 \\
19.0 \\
0.00 \\
0.00 \\
0.16 \\
0.00 \\
11.2 \\
0.09\end{array}$ & $\begin{array}{c}66.2 \\
0.01 \\
16.4 \\
0.02 \\
0.00 \\
0.01 \\
0.00 \\
0.07 \\
15.1\end{array}$ \\
\hline Total & 98.2 & 99.4 & 100.0 & 101.1 & 99.1 & 98.7 & 98.5 & 97.8 \\
\hline $\begin{array}{l}\mathrm{Si} \\
\mathrm{Ti} \\
\mathrm{Al} \\
\mathrm{Fe} \\
\mathrm{Mg} \\
\mathrm{Ca} \\
\mathrm{Ea} \\
\mathrm{Na} \\
\mathrm{K}\end{array}$ & $\begin{array}{l}3.11 \\
0.00 \\
0.89 \\
0.00 \\
0.00 \\
0.00 \\
0.00 \\
0.00 \\
0.89\end{array}$ & $\begin{array}{l}\quad \text { Uni } \\
2.98 \\
0.00 \\
1.02 \\
0.00 \\
0.00 \\
0.01 \\
0.00 \\
1.01 \\
0.00\end{array}$ & $\begin{array}{c}\text { Cell Co } \\
3.02 \\
0.00 \\
0.98 \\
0.00 \\
0.00 \\
0.00 \\
0.00 \\
0.94 \\
0.03\end{array}$ & $\begin{array}{c}\text { osition } \\
3.00 \\
0.00 \\
1.01 \\
0.00 \\
0.00 \\
0.01 \\
0.00 \\
0.97 \\
0.00\end{array}$ & $\begin{array}{c}\text { Based on } \\
3.10 \\
0.00 \\
0.89 \\
0.00 \\
0.00 \\
0.00 \\
0.00 \\
0.01 \\
0.89\end{array}$ & $\begin{array}{l}8(0) \\
3.10 \\
0.00 \\
0.90 \\
0.00 \\
0.00 \\
0.00 \\
0.00 \\
0.00 \\
0.88\end{array}$ & $\begin{array}{l}3.01 \\
0.00 \\
0.99 \\
0.00 \\
0.00 \\
0.01 \\
0.00 \\
0.96 \\
0.01\end{array}$ & $\begin{array}{l}3.09 \\
0.00 \\
0.90 \\
0.00 \\
0.00 \\
0.00 \\
0.00 \\
0.01 \\
0.90\end{array}$ \\
\hline $\begin{array}{l}\text { Tet. } \\
\text { other }\end{array}$ & $\begin{array}{l}4.00 \\
0.89\end{array}$ & $\begin{array}{l}4.00 \\
1.01\end{array}$ & $\begin{array}{c}\text { Coordi } \\
4.00 \\
0.97\end{array}$ & $\begin{array}{c}\text { tion of } \\
4.00 \\
0.98\end{array}$ & $\begin{array}{r}\text { Cations } \\
4.00 \\
0.90\end{array}$ & $\begin{array}{l}4.00 \\
0.89\end{array}$ & $\begin{array}{l}4.00 \\
0.97\end{array}$ & $\begin{array}{l}4.00 \\
0.91\end{array}$ \\
\hline & 3.48 & 2.93 & 3.09 & $\begin{array}{c}\mathrm{Si} /(\mathrm{A})+\mathrm{F} \\
2.97\end{array}$ & 3.48 & 3.42 & 3.04 & 3.42 \\
\hline & 1.00 & 0.99 & $\begin{array}{c}(\mathrm{Al}+\mathrm{Fe}) \\
1.01\end{array}$ & $\begin{array}{c}2 \mathrm{Mg}+2 \mathrm{Ca} \\
1.02\end{array}$ & $\begin{array}{c}-2 \mathrm{Ba}+\mathrm{Na}+\mathrm{K} \\
0.98\end{array}$ & 1.02 & 1.01 & 1.00 \\
\hline $\begin{array}{l}\mathrm{K} \\
\mathrm{Na} \\
\mathrm{Ca}+\mathrm{Mg}\end{array}$ & $\begin{array}{r}99.6 \\
0.4 \\
0.0\end{array}$ & $\begin{array}{r}0.1 \\
99.1 \\
0.8\end{array}$ & $\begin{array}{r}3.3 \\
96.7 \\
0.0\end{array}$ & $\begin{array}{r}0.3 \\
99.1 \\
0.7\end{array}$ & $\begin{array}{r}98.9 \\
0.7 \\
0.4\end{array}$ & $\begin{array}{r}99.6 \\
0.4 \\
0.0\end{array}$ & $\begin{array}{r}0.5 \\
98.7 \\
0.8\end{array}$ & $\begin{array}{r}99.2 \\
0.7 \\
0.1\end{array}$ \\
\hline
\end{tabular}

$\overline{\text { anit symbols }}$ defined in Table $I$. 
APPENDIX H (cont)

Drill Hole USW G-1

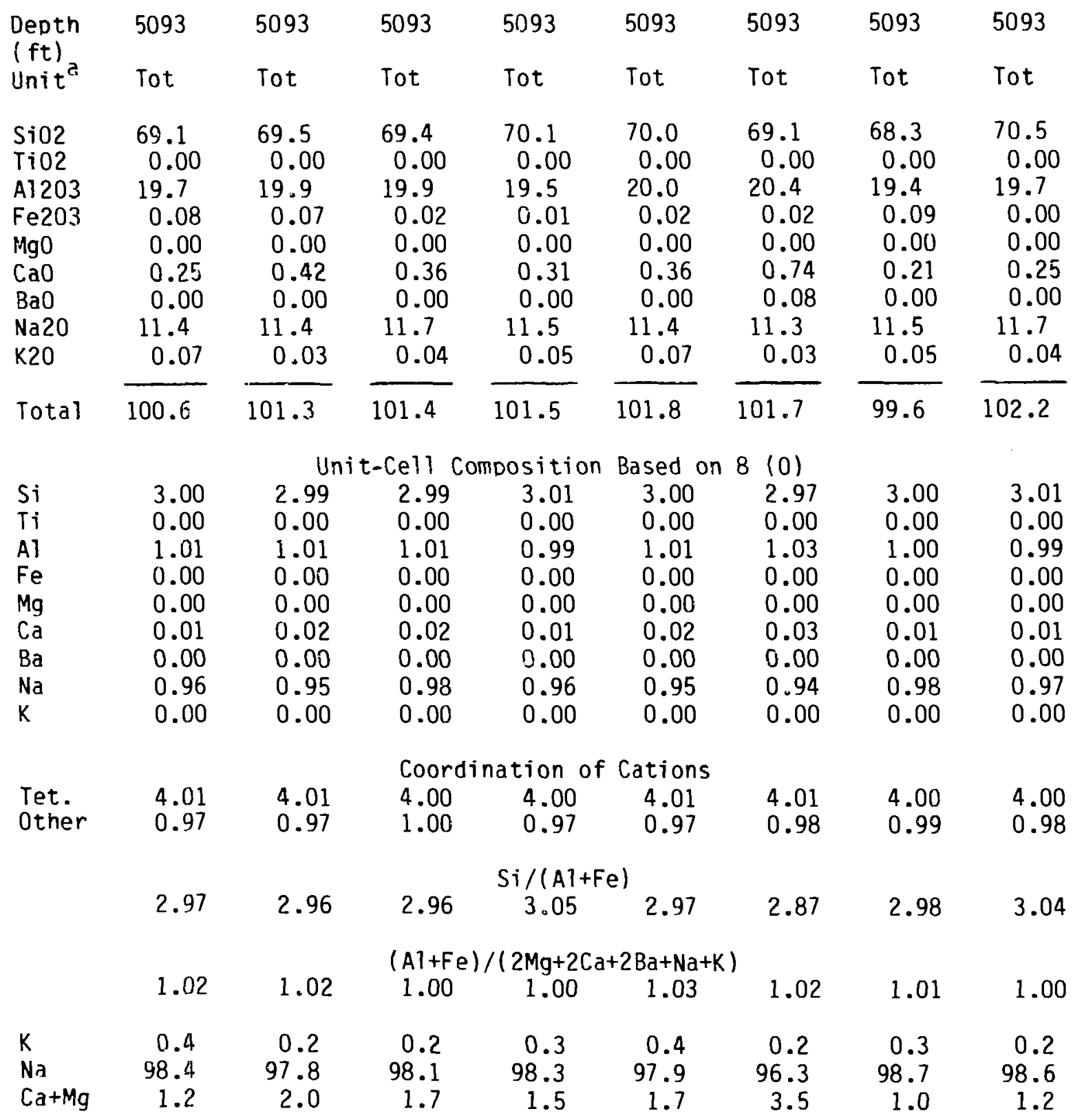


APPENDIX $H$ (cont)

Drill Hole USW G-1

\begin{tabular}{|c|c|c|c|c|c|c|c|c|}
\hline $\begin{array}{l}\text { Dedth } \\
\text { (ft) } \\
\text { Unit }\end{array}$ & 5126 & 5126 & 5296 & 5311 & 5311 & 5412 & 5412 & 5412 \\
\hline $\begin{array}{l}\mathrm{SiO} 02 \\
\mathrm{Ti02} \\
\mathrm{Al} 203 \\
\mathrm{Fe} 203 \\
\mathrm{Mg0} \\
\mathrm{CaO} \\
\mathrm{BaO} \\
\mathrm{Na} 20 \\
\mathrm{~K} 20\end{array}$ & $\begin{array}{c}59.0 \\
0.04 \\
19.3 \\
0.01 \\
0.00 \\
0.00 \\
0.05 \\
11.8 \\
0.02\end{array}$ & $\begin{array}{c}67.8 \\
0.02 \\
17.3 \\
0.13 \\
0.03 \\
0.01 \\
0.05 \\
0.10 \\
16.2\end{array}$ & $\begin{array}{c}68.4 \\
0.00 \\
19.7 \\
0.04 \\
0.00 \\
0.28 \\
0.00 \\
11.6 \\
0.04\end{array}$ & $\begin{array}{c}69.5^{\circ} \\
0.03 \\
19.1 \\
0.04 \\
0.00 \\
0.34 \\
0.13 \\
11.5 \\
0.09\end{array}$ & $\begin{array}{c}68.6 \\
0.01 \\
19.6 \\
0.07 \\
0.01 \\
0.55 \\
0.00 \\
11.5 \\
0.06\end{array}$ & $\begin{array}{c}66.4 \\
0.07 \\
16.8 \\
0.04 \\
0.00 \\
0.02 \\
0.00 \\
0.32 \\
15.0\end{array}$ & $\begin{array}{c}67.4 \\
0.02 \\
16.5 \\
0.01 \\
0.02 \\
0.02 \\
0.10 \\
0.05 \\
15.5\end{array}$ & $\begin{array}{c}68.3 \\
0.00 \\
19.4 \\
0.01 \\
0.00 \\
0.02 \\
0.00 \\
11.9 \\
0.09\end{array}$ \\
\hline Total & 90.2 & 101.6 & 100.1 & 100.7 & 100.4 & 98.7 & 99.6 & 99.7 \\
\hline $\begin{array}{l}\mathrm{Si} \\
\mathrm{Ti} \\
\mathrm{Al} \\
\mathrm{Fe} \\
\mathrm{Mg} \\
\mathrm{Ca} \\
\mathrm{Ba} \\
\mathrm{Na} \\
\mathrm{K}\end{array}$ & $\begin{array}{l}2.88 \\
0.00 \\
1.11 \\
0.00 \\
0.00 \\
0.00 \\
0.00 \\
1.12 \\
0.00\end{array}$ & $\begin{array}{l}3.07 \\
0.00 \\
0.92 \\
0.00 \\
0.00 \\
0.00 \\
0.00 \\
0.01 \\
0.93\end{array}$ & $\begin{array}{c}\text { Unit-Cel1 } \\
2.99 \\
0.00 \\
1.01 \\
0.00 \\
0.00 \\
0.01 \\
0.00 \\
0.98 \\
0.00\end{array}$ & $\begin{array}{c}\text { Composit } \\
3.01 \\
0.00 \\
0.98 \\
0.00 \\
0.00 \\
0.02 \\
0.00 \\
0.97 \\
0.00\end{array}$ & 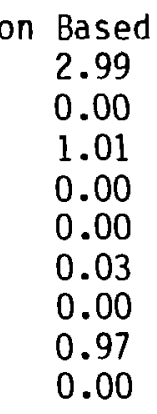 & on $\begin{array}{l}8 \quad(0) \\
3.08 \\
0.00 \\
0.92 \\
0.00 \\
0.00 \\
0.00 \\
0.00 \\
0.03 \\
0.89\end{array}$ & $\begin{array}{l}3.10 \\
0.00 \\
0.89 \\
0.00 \\
0.00 \\
0.00 \\
0.00 \\
0.00 \\
0.91\end{array}$ & $\begin{array}{l}2.99 \\
0.00 \\
1.00 \\
0.00 \\
0.00 \\
0.00 \\
0.00 \\
1.01 \\
0.01\end{array}$ \\
\hline $\begin{array}{l}\text { Tet. } \\
\text { Other }\end{array}$ & $\begin{array}{l}4.00 \\
1.12\end{array}$ & $\begin{array}{l}3.99 \\
0.95\end{array}$ & $\begin{array}{c}\text { Coordi } \\
4.00 \\
1.00\end{array}$ & $\begin{array}{c}\text { ation of } \\
3.99 \\
0.99\end{array}$ & $\begin{array}{r}\text { Cations } \\
4.00 \\
1.00\end{array}$ & $\begin{array}{l}4.00 \\
0.92\end{array}$ & $\begin{array}{l}3.99 \\
0.92\end{array}$ & $\begin{array}{l}4.00 \\
1.02\end{array}$ \\
\hline \multicolumn{9}{|c|}{$\mathrm{Si} /(\mathrm{A})+\mathrm{Fe})$} \\
\hline & \multicolumn{7}{|c|}{$(\mathrm{A}]+\mathrm{Fe}) /(2 \mathrm{Mg}+2 \mathrm{Ca}+2 \mathrm{Ba}+\mathrm{Na}+\mathrm{K})$} & 0.98 \\
\hline $\begin{array}{l}\mathrm{K} \\
\mathrm{Na} \\
\mathrm{Ca}+\mathrm{Mg}\end{array}$ & $\begin{array}{r}0.1 \\
99.9 \\
0.0\end{array}$ & $\begin{array}{r}98.8 \\
0.9 \\
0.3\end{array}$ & $\begin{array}{r}0.2 \\
98.5 \\
1.3\end{array}$ & $\begin{array}{r}0.5 \\
97.9 \\
1.6\end{array}$ & $\begin{array}{r}0.3 \\
97.0 \\
2.6\end{array}$ & $\begin{array}{r}96.8 \\
3.1 \\
0.1\end{array}$ & $\begin{array}{r}99.3 \\
0.5 \\
0.3\end{array}$ & $\begin{array}{r}0.5 \\
99.4 \\
0.1\end{array}$ \\
\hline
\end{tabular}


APPENDIX $\mathrm{H}$ (cont)

Drill Hole USW G-1

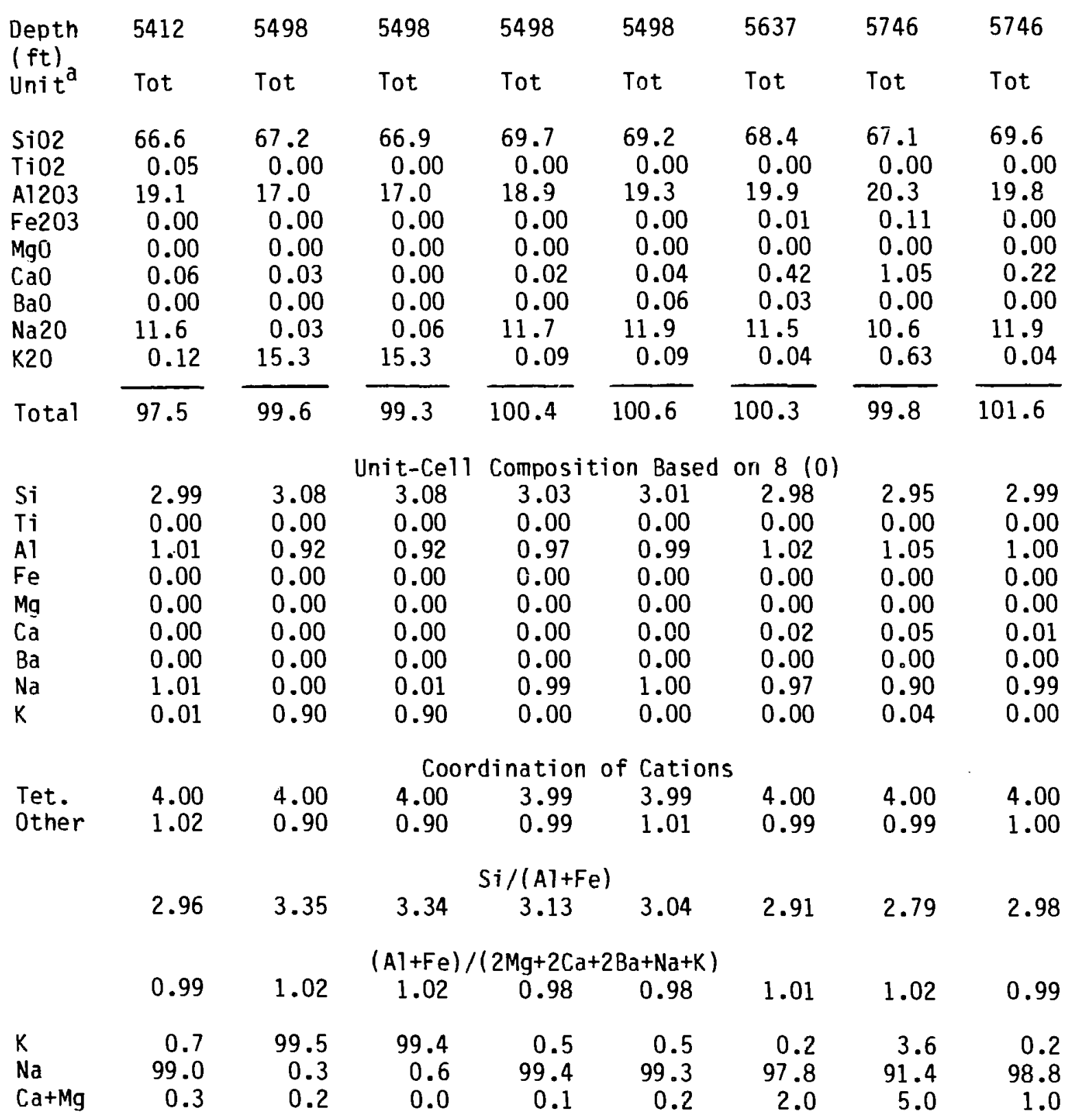




\section{APPENDIX $H$ (cont)}

\section{Drill Hole USW G-1}

\begin{tabular}{|c|c|c|c|c|c|c|c|c|}
\hline Depth & 5746 & 5746 & 5746 & 5847 & 5847 & 5847 & 5847 & 5947 \\
\hline Unit ${ }^{a}$ & Tot & Tot & Tot & Tot & Tot & Tot & Tot & Tot \\
\hline $\begin{array}{l}\mathrm{SiO} 2 \\
\mathrm{Ti} 02 \\
\mathrm{Al} 203 \\
\mathrm{Fe} 203 \\
\mathrm{MgO} \\
\mathrm{CaO} \\
\mathrm{BaO} \\
\mathrm{Na} 20 \\
\mathrm{~K} 20\end{array}$ & $\begin{array}{c}66.4 \\
0.00 \\
20.1 \\
0.00 \\
0.00 \\
1.13 \\
0.00 \\
10.3 \\
0.02\end{array}$ & $\begin{array}{c}69.1 \\
0.00 \\
19.8 \\
0.04 \\
0.00 \\
0.38 \\
0.00 \\
11.5 \\
0.04\end{array}$ & $\begin{array}{c}68.4 \\
0.00 \\
19.6 \\
0.03 \\
0.00 \\
0.17 \\
0.00 \\
11.3 \\
0.07\end{array}$ & $\begin{array}{c}69.8 \\
0.04 \\
18.8 \\
0.04 \\
0.00 \\
0.13 \\
0.05 \\
11.3 \\
0.13\end{array}$ & $\begin{array}{c}68.4 \\
0.03 \\
19.6 \\
0.05 \\
0.02 \\
0.62 \\
0.15 \\
11.5 \\
0.09\end{array}$ & $\begin{array}{c}67.2 \\
0.03 \\
19.5 \\
0.08 \\
0.00 \\
1.24 \\
0.20 \\
10.8 \\
0.10\end{array}$ & $\begin{array}{c}68.6 \\
0.01 \\
19.5 \\
0.00 \\
0.01 \\
0.95 \\
0.00 \\
11.1 \\
0.07\end{array}$ & $\begin{array}{c}67.3 \\
0.00 \\
20.0 \\
0.01 \\
0.00 \\
0.80 \\
0.00 \\
11.1 \\
0.01\end{array}$ \\
\hline Total & 98.4 & 100.9 & 99.6 & 100.3 & 100.5 & 99.1 & 100.2 & 99.2 \\
\hline $\begin{array}{l}\mathrm{Si} \\
\mathrm{Ti} \\
\mathrm{Al} \\
\mathrm{Fe} \\
\mathrm{Mg} \\
\mathrm{Ca} \\
\mathrm{Ba} \\
\mathrm{Na} \\
\mathrm{K}\end{array}$ & $\begin{array}{l}2.95 \\
0.00 \\
1.05 \\
0.00 \\
0.00 \\
0.05 \\
0.00 \\
0.93 \\
0.00\end{array}$ & $\begin{array}{l}\quad \text { Un } \\
2.99 \\
0.00 \\
1.01 \\
0.00 \\
0.00 \\
0.02 \\
0.00 \\
0.97 \\
0.00\end{array}$ & $\begin{array}{c}-C e 11 c C \\
3.00 \\
0.00 \\
1.01 \\
0.00 \\
0.00 \\
0.01 \\
0.00 \\
0.96 \\
0.00\end{array}$ & $\begin{array}{c}\text { mpositior } \\
3.03 \\
0.00 \\
0.96 \\
0.00 \\
0.00 \\
0.01 \\
0.00 \\
0.95 \\
0.01\end{array}$ & $\begin{array}{c}\text { Based or } \\
2.98 \\
0.00 \\
1.01 \\
0.00 \\
0.00 \\
0.03 \\
0.00 \\
0.97 \\
0.01\end{array}$ & $\begin{array}{ll}8(0) \\
2.97 \\
0.00 \\
1.02 \\
0.00 \\
0.00 \\
0.06 \\
0.00 \\
0.93 \\
0.01\end{array}$ & $\begin{array}{l}2.99 \\
0.00 \\
1.00 \\
0.00 \\
0.00 \\
0.04 \\
0.00 \\
0.94 \\
0.60\end{array}$ & $\begin{array}{l}2.96 \\
0.00 \\
1.04 \\
0.00 \\
0.00 \\
0.04 \\
0.00 \\
0.95 \\
0.00\end{array}$ \\
\hline $\begin{array}{l}\text { Tet. } \\
\text { Other }\end{array}$ & $\begin{array}{l}4.00 \\
0.99\end{array}$ & $\begin{array}{l}4.00 \\
0.98\end{array}$ & $\begin{array}{l}\quad \text { Coo } \\
4.01 \\
0.97\end{array}$ & $\begin{array}{c}\text { dination } \\
4.00 \\
0.97\end{array}$ & $\begin{array}{c}\text { of Catio } \\
3.99 \\
1.01\end{array}$ & $\begin{array}{l}3.99 \\
0.99\end{array}$ & $\begin{array}{r}3.99 \\
0.99\end{array}$ & $\begin{array}{l}4.00 \\
0.99\end{array}$ \\
\hline & 2.80 & 2.96 & 2.96 & $\begin{array}{l}\mathrm{Si} / / \\
3.14\end{array}$ & $\begin{array}{l}+\mathrm{Fe}) \\
2.96\end{array}$ & 2.92 & 2.98 & 2.85 \\
\hline & 1.01 & 1.01 & $1.03^{1 /}$ & $\begin{array}{c}\mathrm{Fe}) /(2 \mathrm{M} \\
0.99\end{array}$ & $\begin{array}{c}2 \mathrm{Ca}+2 \mathrm{Ba} \\
0.97\end{array}$ & $\begin{array}{l}(\mathrm{a}+\mathrm{K}) \\
0.97\end{array}$ & 0.97 & 1.01 \\
\hline $\begin{array}{l}\mathrm{K} \\
\mathrm{Na} \\
\mathrm{Ca}+\mathrm{Mg}\end{array}$ & $\begin{array}{r}0.1 \\
94.4 \\
5.5\end{array}$ & $\begin{array}{r}0.2 \\
98.0 \\
1.8\end{array}$ & $\begin{array}{r}0.4 \\
98.8 \\
0.8\end{array}$ & $\begin{array}{r}0.7 \\
98.6 \\
0.6\end{array}$ & $\begin{array}{r}0.5 \\
96.5 \\
3.0\end{array}$ & $\begin{array}{r}0.6 \\
93.5 \\
5.9\end{array}$ & $\begin{array}{r}0.4 \\
95.0 \\
4.6\end{array}$ & $\begin{array}{r}0.1 \\
96.1 \\
3.8\end{array}$ \\
\hline
\end{tabular}


APPENDIX $H$ (cont)

Drill Hole USW G-2

\begin{tabular}{|c|c|c|c|c|c|c|c|c|}
\hline Depth & 2325 & 2325 & 2325 & 2325 & 2430 & 4805 & 4805 & 4805 \\
\hline Unit ${ }^{a}$ & Tht & Tht & Tht & Tht & Tht & $\mathrm{Tl} r$ & $\operatorname{Tl} r$ & $T 1 r$ \\
\hline $\begin{array}{l}\mathrm{SiO} 2 \\
\mathrm{TiO} 2 \\
\mathrm{~A} 1203 \\
\mathrm{Fe} 203 \\
\mathrm{MgO} \\
\mathrm{CaO} \\
\mathrm{BaO} \\
\mathrm{Na} 20 \\
\mathrm{~K} 20\end{array}$ & $\begin{array}{c}67.9 \\
0.00 \\
16.5 \\
0.03 \\
0.00 \\
0.02 \\
0.00 \\
0.03 \\
15.5\end{array}$ & $\begin{array}{c}68.1 \\
0.04 \\
16.4 \\
0.00 \\
0.00 \\
0.00 \\
0.00 \\
0.04 \\
15.3\end{array}$ & $\begin{array}{c}68.7 \\
0.00 \\
16.6 \\
0.00 \\
0.00 \\
0.02 \\
0.00 \\
0.18 \\
15.1\end{array}$ & $\begin{array}{c}68.8 \\
0.00 \\
16.4 \\
0.00 \\
0.00 \\
0.00 \\
0.00 \\
0.05 \\
15.2\end{array}$ & $\begin{array}{c}67.8 \\
0.01 \\
16.8 \\
0.03 \\
0.00 \\
0.00 \\
0.00 \\
0.03 \\
15.5\end{array}$ & $\begin{array}{c}65.5 \\
0.00 \\
20.7 \\
0.10 \\
0.00 \\
1.55 \\
0.08 \\
10.7 \\
0.39\end{array}$ & $\begin{array}{c}65.6 \\
0.00 \\
16.7 \\
0.01 \\
0.00 \\
0.00 \\
0.18 \\
0.32 \\
15.2\end{array}$ & $\begin{array}{c}66.9 \\
0.00 \\
16.5 \\
0.00 \\
0.00 \\
0.00 \\
0.10 \\
0.17 \\
15.1\end{array}$ \\
\hline Total & 99.9 & 99.9 & 100.6 & 100.4 & 100.1 & 98.9 & 98.0 & 98.7 \\
\hline $\begin{array}{l}\mathrm{Si} \\
\mathrm{Ti} \\
\mathrm{Al} \\
\mathrm{Fe} \\
\mathrm{Mg} \\
\mathrm{Ca} \\
\mathrm{Ba} \\
\mathrm{Na} \\
\mathrm{K}\end{array}$ & $\begin{array}{l}3.11 \\
0.00 \\
0.89 \\
0.00 \\
0.00 \\
0.00 \\
0.00 \\
0.00 \\
0.90\end{array}$ & $\begin{array}{l}3.11 \\
0.00 \\
0.89 \\
0.00 \\
0.00 \\
0.00 \\
0.00 \\
0.00 \\
0.89\end{array}$ & $\begin{array}{c}\text { it-Cel1 } \\
3.11 \\
0.00 \\
0.89 \\
0.00 \\
0.00 \\
0.00 \\
0.00 \\
0.02 \\
0.87\end{array}$ & $\begin{array}{c}\text { Composit } \\
3.12 \\
0.00 \\
0.88 \\
0.00 \\
0.00 \\
0.00 \\
0.00 \\
0.00 \\
0.88\end{array}$ & $\begin{array}{c}\text { on Based } \\
3.10 \\
0.00 \\
0.90 \\
0.00 \\
0.00 \\
0.00 \\
0.00 \\
0.00 \\
0.90\end{array}$ & $\begin{array}{l}8(0) \\
2.91 \\
0.00 \\
1.08 \\
0.00 \\
0.00 \\
0.07 \\
0.00 \\
0.92 \\
0.02\end{array}$ & $\begin{array}{l}3.07 \\
0.00 \\
0.92 \\
0.00 \\
0.00 \\
0.00 \\
0.00 \\
0.03 \\
0.91\end{array}$ & $\begin{array}{l}3.10 \\
0.00 \\
0.90 \\
0.00 \\
0.00 \\
0.00 \\
0.00 \\
0.02 \\
0.89\end{array}$ \\
\hline $\begin{array}{l}\text { Tet. } \\
\text { Other }\end{array}$ & $\begin{array}{l}4.00 \\
0.91\end{array}$ & $\begin{array}{l}4.00 \\
0.89\end{array}$ & $\begin{array}{l}\text { Coo } \\
4.00 \\
0.89\end{array}$ & $\begin{array}{c}\text { dination } \\
4.00 \\
0.88\end{array}$ & $\begin{array}{r}\text { of Cati } \\
4.00 \\
0.91\end{array}$ & $\begin{array}{l}4.00 \\
1.02\end{array}$ & $\begin{array}{l}3.99 \\
0.94\end{array}$ & $\begin{array}{l}4.00 \\
0.91\end{array}$ \\
\hline & 3.49 & 3.52 & 3.51 & $\begin{array}{c}\mathrm{Si} / 1 \\
3.56\end{array}$ & $\begin{array}{l}(+F e) \\
3.43\end{array}$ & 2.68 & 3.33 & 3.44 \\
\hline & 0.98 & 0.99 & $0.99^{1 A}$ & $\begin{array}{c}+F e) /(2 \mathrm{~N} \\
0.99\end{array}$ & $\begin{array}{r}+2 \mathrm{Ca}+2 \mathrm{~B} \\
1.00\end{array}$ & $\begin{array}{l}(a+K) \\
\quad 1.00\end{array}$ & 0.97 & 0.99 \\
\hline $\begin{array}{l}\mathrm{K} \\
\mathrm{Na} \\
\mathrm{Ca}+\mathrm{Mg}\end{array}$ & $\begin{array}{r}99.6 \\
0.3 \\
0.1\end{array}$ & $\begin{array}{r}99.6 \\
0.4 \\
0.0\end{array}$ & $\begin{array}{r}98.1 \\
1.8 \\
0.1\end{array}$ & $\begin{array}{r}99.5 \\
0.5 \\
0.0\end{array}$ & $\begin{array}{r}99.7 \\
0.3 \\
0.0\end{array}$ & $\begin{array}{r}2.2 \\
90.5 \\
7.3\end{array}$ & $\begin{array}{r}96.9 \\
3.1 \\
0.0\end{array}$ & $\begin{array}{r}98.3 \\
1.7 \\
0.0\end{array}$ \\
\hline
\end{tabular}




\section{APPENDIX $H$ (cont)}

\section{Drill Hole USW G-2}

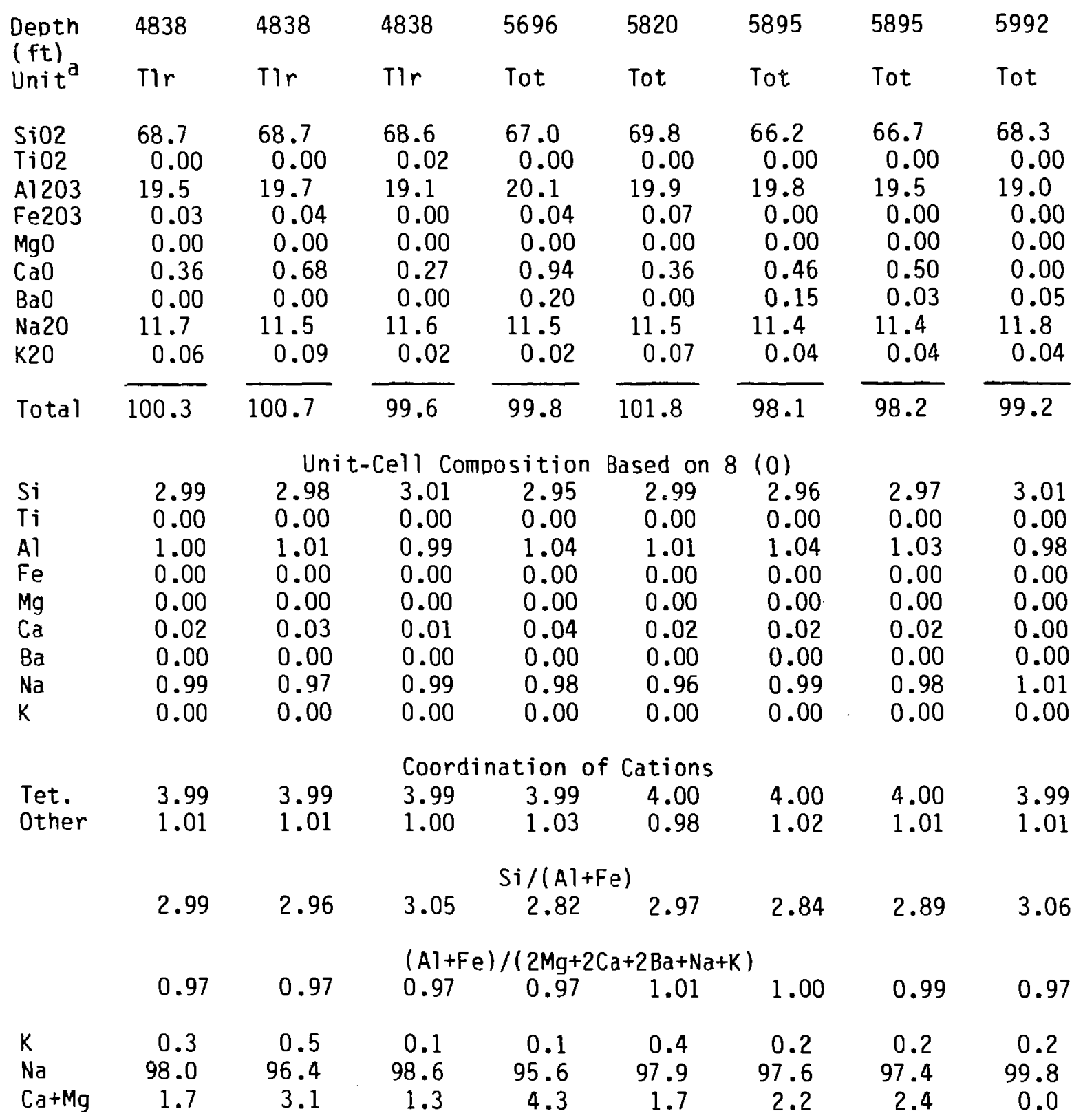


APPENDIX $H($ cont $)$

Drill Hole USH G-2

\begin{tabular}{|c|c|}
\hline $\begin{array}{l}\text { Depth } \\
(\mathrm{ft}) \\
\text { Unit }\end{array}$ & $\begin{array}{l}5992 \\
\text { Tot }\end{array}$ \\
\hline $\begin{array}{l}\mathrm{SiO} 2 \\
\mathrm{TiO} 2 \\
\mathrm{~A} 1203 \\
\mathrm{Fe} 203 \\
\mathrm{MgO} \\
\mathrm{CaO} \\
\mathrm{BaO} \\
\mathrm{Na} 20 \\
\mathrm{~K} 20\end{array}$ & $\begin{array}{c}63.5 \\
0.02 \\
18.0 \\
0.06 \\
0.00 \\
0.08 \\
1.05 \\
0.42 \\
15.5\end{array}$ \\
\hline Total & 98.7 \\
\hline $\begin{array}{l}\text { Unit-Cell } \\
8(0) \\
\mathrm{Si} \\
\mathrm{Ti} \\
\mathrm{Al} \\
\mathrm{Fe} \\
\mathrm{Mg} \\
\mathrm{Ca} \\
\mathrm{Ba} \\
\mathrm{Na} \\
\mathrm{K}\end{array}$ & $\begin{array}{l}\text { Composition Based on } \\
2.99 \\
0.00 \\
1.00 \\
0.00 \\
0.00 \\
0.00 \\
0.02 \\
0.04 \\
0.93\end{array}$ \\
\hline $\begin{array}{l}\text { Coordinat } \\
\text { Tet. } \\
\text { other }\end{array}$ & $\begin{array}{l}\text { tion of Cations } \\
4.00 \\
1.00\end{array}$ \\
\hline $\mathrm{Si} /(\mathrm{A}$ & $\begin{array}{r}\mathrm{A} 1+\mathrm{Fe}) \\
2.98\end{array}$ \\
\hline$(\mathrm{A})+\mathrm{Fe}) /($ & $\begin{array}{l}2 \mathrm{Mg}+2 \mathrm{Ca}+2 \mathrm{Ba}+\mathrm{Na}+\mathrm{K}) \\
0.98\end{array}$ \\
\hline $\begin{array}{l}\mathrm{K} \\
\mathrm{Na} \\
\mathrm{Ca}+\mathrm{Mg}\end{array}$ & $\begin{array}{r}95.7 \\
3.9 \\
0.4\end{array}$ \\
\hline
\end{tabular}




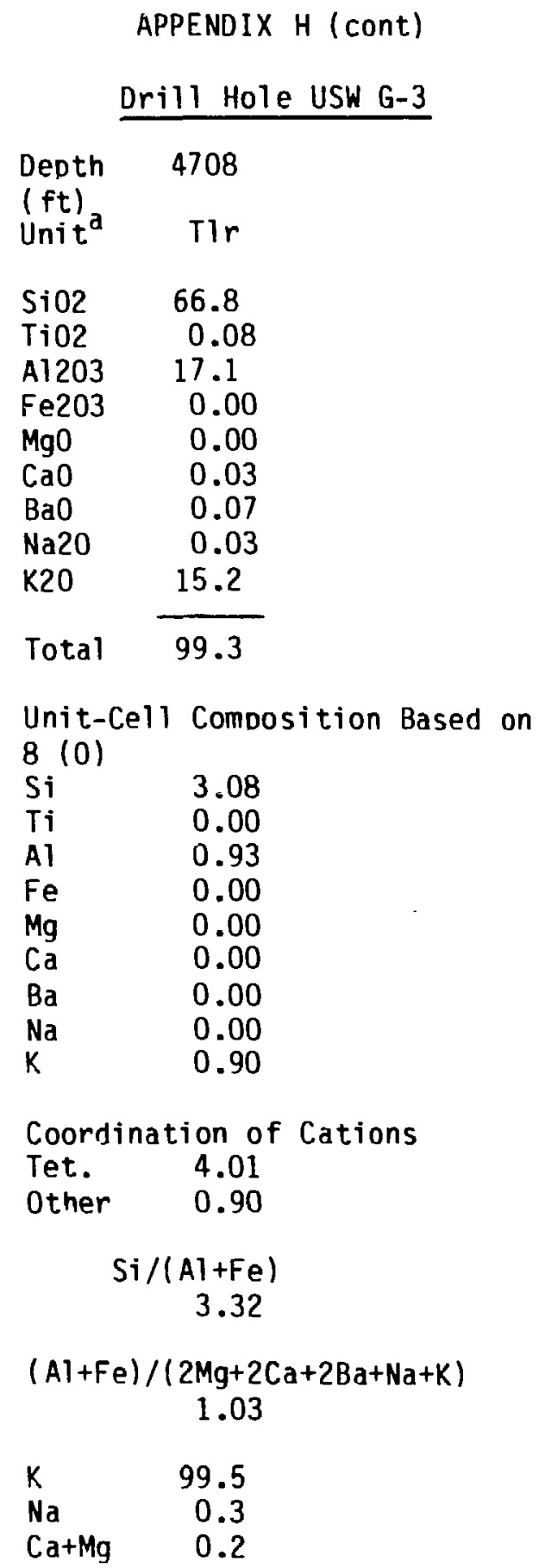




\section{APPENDIX $H$ (cont) \\ Drill Hole USW G-4}

\begin{tabular}{|c|c|}
\hline $\begin{array}{l}\text { Depth } \\
\text { (ft) } \\
\text { Unit }\end{array}$ & $\begin{array}{r}2738 \\
T c b\end{array}$ \\
\hline $\begin{array}{l}\mathrm{SiO} 2 \\
\mathrm{TiO} 02 \\
\mathrm{Al} 203 \\
\mathrm{Fe} 203 \\
\mathrm{MgO} \\
\mathrm{CaO} \\
\mathrm{BaO} \\
\mathrm{Na2O} \\
\mathrm{K} 20\end{array}$ & $\begin{array}{c}66.4 \\
0.00 \\
17.7 \\
0.00 \\
0.00 \\
0.03 \\
0.05 \\
0.06 \\
16.1\end{array}$ \\
\hline Total & 100.4 \\
\hline $\begin{array}{l}\text { Unit-C } \\
8 \text { ( }(0) \\
\mathrm{Si} \\
\mathrm{Ti} \\
\mathrm{Al} \\
\mathrm{Fe} \\
\mathrm{Mg} \\
\mathrm{Ca} \\
\mathrm{Ba} \\
\mathrm{Na} \\
\mathrm{K}\end{array}$ & $\begin{array}{l}11 \text { Composition Based on } \\
3.04 \\
0.00 \\
0.96 \\
0.00 \\
0.00 \\
0.00 \\
0.00 \\
0.01 \\
0.94\end{array}$ \\
\hline $\begin{array}{l}\text { Coord } \\
\text { Tet. } \\
\text { Other }\end{array}$ & $\begin{array}{l}\text { lation of Cations } \\
4.00 \\
0.95\end{array}$ \\
\hline & $\begin{array}{c}i /(A)+F e) \\
3.18\end{array}$ \\
\hline$(A)+F$ & $\begin{array}{l}\text { ) } /(2 \mathrm{Mg}+2 \mathrm{Ca}+2 \mathrm{Ba}+\mathrm{Na}+\mathrm{K}) \\
1.01\end{array}$ \\
\hline $\begin{array}{l}\mathrm{K} \\
\mathrm{Na} \\
\mathrm{Ca}+\mathrm{Mg}\end{array}$ & $\begin{array}{r}99.3 \\
0.6 \\
0.2\end{array}$ \\
\hline
\end{tabular}




\section{APPENDIX H (cont)}

\section{Drill Hole J-13}

\begin{tabular}{|c|c|c|c|}
\hline $\begin{array}{l}\text { Depth } \\
\text { (ft) } \\
\text { Unit }\end{array}$ & 2980 & 2980 & 2980 \\
\hline $\begin{array}{l}\text { Si02 } \\
\text { Ti02 }\end{array}$ & & & 68.3 \\
\hline A1203 & 20.1 & $\begin{array}{c}0.00 \\
19.6\end{array}$ & $\begin{array}{c}0.00 \\
19.6\end{array}$ \\
\hline$=\mathrm{e} 203$ & 0.00 & 0.00 & 0.00 \\
\hline $\mathrm{MgO}$ & 0.00 & 0.00 & 0.00 \\
\hline $\mathrm{CaO}$ & 0.58 & 0.15 & 0.33 \\
\hline $\mathrm{BaO}$ & 0.00 & 0.00 & 0.00 \\
\hline $\mathrm{Na} 20$ & 11.8 & 12.0 & 12.0 \\
\hline K20 & 0.06 & 0.04 & 0.03 \\
\hline otal & 99.8 & 99.0 & 100.3 \\
\hline
\end{tabular}

Unit-Cel1 Composition Based on 8 (0)

$\begin{array}{llll}\mathrm{Si} & 2.95 & 2.97 & 2.98 \\ \mathrm{Ti} & 0.00 & 0.00 & 0.00 \\ \mathrm{Al} & 1.04 & 1.02 & 1.01 \\ \mathrm{Fe} & 0.00 & 0.00 & 0.00 \\ \mathrm{Mg} & 0.00 & 0.00 & 0.00 \\ \mathrm{Ca} & 0.03 & 0.01 & 0.02 \\ \mathrm{Ba} & 0.00 & 0.00 & 0.00 \\ \mathrm{Na} & 1.01 & 1.03 & 1.02 \\ \mathrm{~K} & 0.00 & 0.00 & 0.00\end{array}$

\begin{tabular}{lccc} 
Tet. & 3.99 & 3.99 & 3.99 \\
Other & 1.04 & 1.04 & 1.03 \\
& \multicolumn{3}{c}{$\mathrm{Si} /(\mathrm{A})+\mathrm{Fe})$} \\
& 2.83 & 2.91 & 2.95 \\
& \multicolumn{3}{c}{$(\mathrm{Al}+\mathrm{Fe}) /(2 \mathrm{Mg}+2 \mathrm{Ca}+2 \mathrm{Ba}+\mathrm{Na}+\mathrm{K})$} \\
& 0.98 & 0.98 & 0.96 \\
& & 0.2 & 0.2 \\
$\mathrm{~K}$ & 0.3 & 0.2 & 98.3 \\
$\mathrm{Na}$ & 97.0 & 99.1 & 1.5 \\
$\mathrm{Ca}+\mathrm{Mg}$ & 2.6 & 0.7 &
\end{tabular}


APPENDIX H (cont)

Outcrop at Prow Pass, Northern Yucca Mountain

Sample Number 3-15-82-4 82FB-1

Both samples are nonwelded tuff near base of tuff of Calico Hills. Nevada State Coordinates ( $\mathrm{ft}$ ) for sample locations: $3-15-82-4=786500 \mathrm{~N}, 551000 \mathrm{E}$; $82 \mathrm{FB}-1=789700 \mathrm{~N}, 549700 \mathrm{E}$.

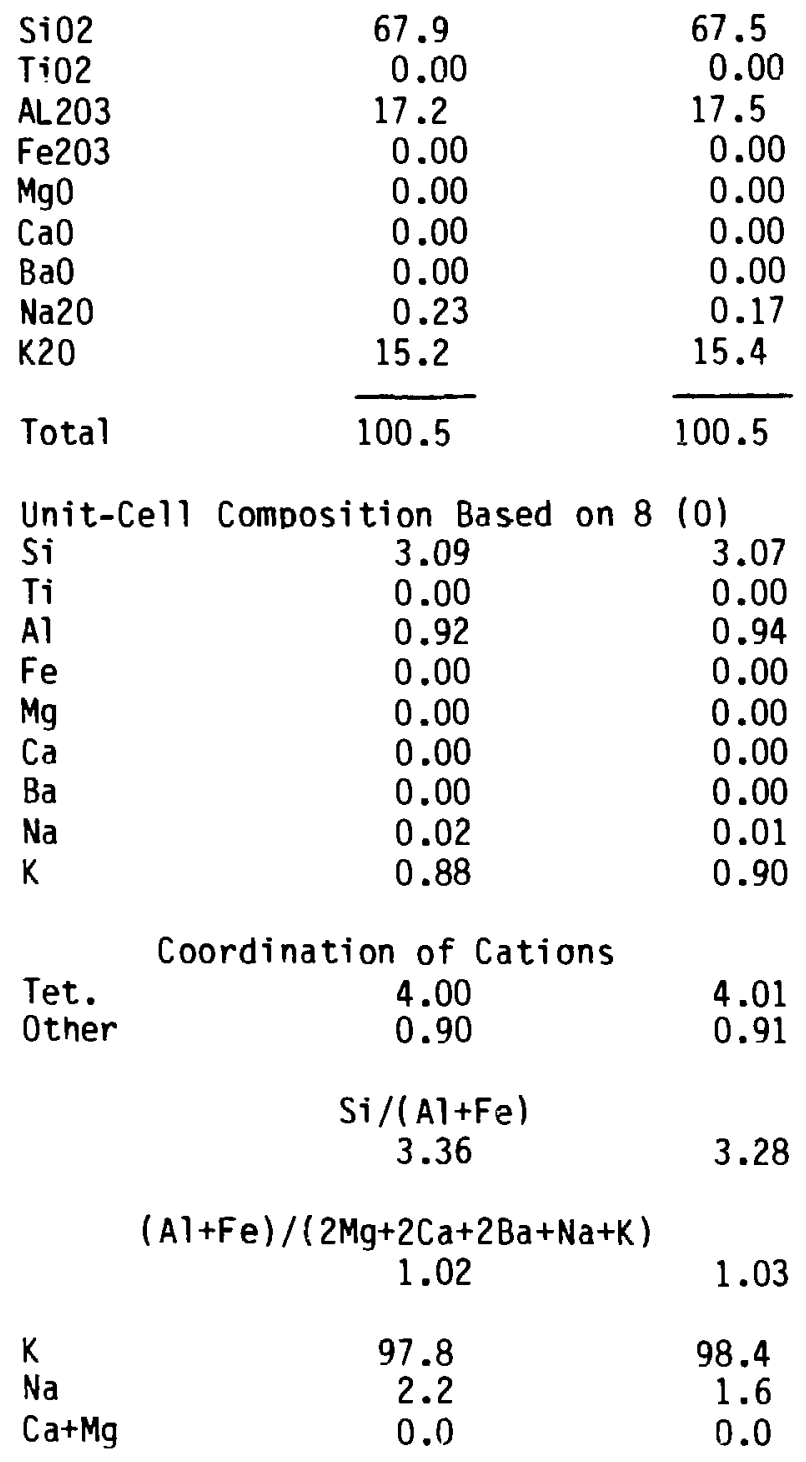

